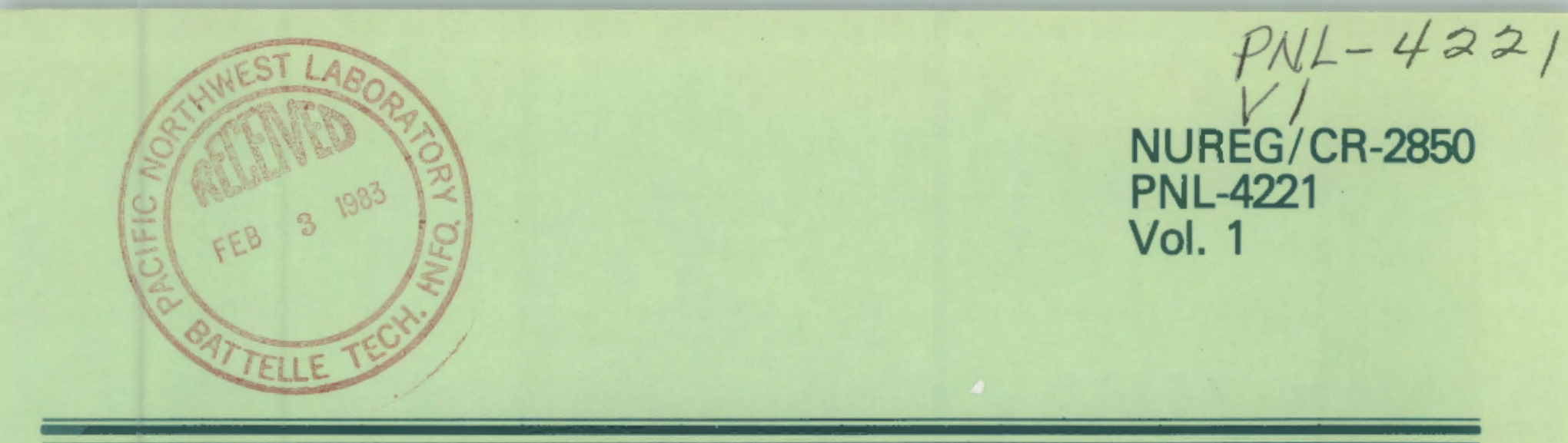

\title{
Population Dose Commitments Due to Radioactive Releases from Nuclear Power Plant Sites in 1979
}

Prepared by D. A. Baker, R. A. Peloquin

Pacific Northwest Laboratory Operated by

Battelle Memorial Institute

Prepared for U.S. Nuclear Regulatory Commission 
This report was prepared as an account of work sponsored by an agency of the United States Government. Neither the United States Government nor any agency thereof, or any of their employees, makes any warranty, expressed or implied, or assumes any legal liability of responsibility for any third party's use, or the results of such use, of any information, apparatus, product or process disclosed in this report, or represents that its use by such third party would not infringe privately owned rights.

\section{Availability of Reference Materials Cited in NRC Publications}

Most documents cited in NRC publications will be available from one of the following sources:

1. The NRC Public Document Room, 1717 H Street, N.W. Washington, DC 20555

2. The NRC/GPO Sales Program, U.S. Nuclear Regulatory Commission, Washington, DC 20555

3. The National Technical Information Service, Springfield, VA 22161

Although the listing that follows represents the majority of documents cited in NRC publications, it is not intended to be exhaustive.

Referenced documents available for inspection and copying for a fee from the NRC Public Document Room include NRC correspondence and irternal NRC memoranda; NRC Office of Inspection and Enforcement bulletins, circulars, information notices, inspection and investigation notices; Licensee Event Reports; vendor reports and correspondence; Commission papers; and applicant and licensee documents and correspondence.

The following documents in the NUREG series are available for purchase from the NRC/GPO Sales Program: formal NRC staff and contractor reports, NRC-sponsored conference proceedings, and NRC booklets and brochures. Also available are Regulatory Guides, NRC regulations in the Code of Federal Regulations, and Nuclear Regulatory Commission Issuances.

Documents available from the National Technical Information Service include NUREG series reports and technical reports prepared by other federal agencies and reports prepared by the Atomic Energy Commission, forerunner agency to the Nuclear Regulatory Commission.

Documents available from public and special technical !ibraries include all open literature items, such as books, journal and periodical articles, and transactions. Federal Register notices, federal and state legisiation, and congressional reports can usually be obtained from these libraries.

Documents such as theses, dissertations, foreign reports and translations, and non-NRC conference proceedings are available for purchase from the organization sponsoring the publication cited.

Single copies of NRC draft reports are available free upon written request to the Division of Technical Information and Document Control, U.S. Nuclear Regulatory Commission, Washington, DC 20555.

Copies of industry codes and standards used in a substantive manner in the NRC regulatory process are maintained at the NRC Library, 7920 Norfolk Avenue, Bethesda, Maryland, and are available there for reference use by the public. Codes and standards are usually copyrighted and may be purchased from the originating organization or, if they are American National Standards, from the American National Standards Institute. 1430 Broadway, New York, NY 10018. 
NUREG/CR-2850

PNL-4221

Vol. 1

\section{Population Dose Commitments Due to Radioactive Releases from Nuclear Power Plant Sites in 1979}

Manuscript Completed: July 1982

Date Published: December 1982

Prepared by

D. A. Baker, R. A. Peloquin

Pacific Northwest Laboratory

Richland, WA 99352

\section{Prepared for}

Division of Data Automation and Management Information

Office of Resource Management

U.S. Nuclear Regulatory Commission

Washington, D.C. 20555

NRC FIN B2243 



\section{PREFACE}

NUREG/CR-2850, Volume 1, PNL-4221, "Population Dose Commitments Due To Radioactive Releases From Nuclear Power Plant Sites in 1979" is an annual report with a permanent identifier. To differentiate one year from another, only the volume number will change. The NUREG number will remain the same during the lifetime of the report series. 



\section{PREVIOUS REPORTS IN THIS SERIES}

1. Population Dose Comm Itments Due to Radloactive Releases from Nuclear Power Plant Sites in 1975, PNL-2439, October 1977.

2. Population Dose Commitments Due to Radloactive Releases from Nuclear Power Plant Sites in 1976, NUREG/CR-1125, PNL-2940, December 1979.

3. Population Dose Comm itments Due to Radloactive Releases from Nuclear Power Plant Sites in 1977, NUREG/CR-1498, PNL-3324, October 1980.

4. Population Dose Commitments Due to Radioactlve Releases from Nuclear Power Plant Sites in 1978, NUREG/CR-2201, PNL-4039, June 1982. 



\section{ABSTRACT}

Population radiation dose commitments have been estimated from reported radionuclide releases from commercial power reactors operating during 1979. Fifty-year dose commitments from a one-year exposure were calculated from both I Iquid and atmospheric releases for four population groups (Infant, chlld, teen-ager and adult) residing between 2 and $80 \mathrm{~km}$ from each site. This report tabulates the results of these calculations, showing the dose commitments for both liquid and airborne pathways for each age group and organ. Also Included for each site is a histogram show ing the fraction of the total population within 2 to $80 \mathrm{~km}$ around each site receiving varlous average dose commitments from the alrborne pathways.

The total dose commitment from both I lquid and alrborne pathways ranged from a high of 1300 person-rem to a low of 0.0002 person-rem with an arithmetic mean of 38 person-rem. The total population dose for all sites was estimated at 1800 person-rem for the 94 million people considered at risk.

The average individual dose commitment from all pathways on a site basls ranged from a low of $2 \times 10^{-6} \mathrm{mrem}$ to a high of $0.7 \mathrm{mrem}$. No attempt was made in this study to determine the maximum dose commitment recelved by any one individual from the radionuclides released at any of the sites. 



\section{CONTENTS}

ABSTRACT.............................. . Ii

INTRODUCTION . . . . . . . . . . . . . . . . . . . . 1

Site Dependent Parameters . . . . . . . . . . . 2

RESULTS ......................... 7

SITE SUMMARIES ......................... 11

Arkansas 0ne $1 \& 2$ \& . . . . . . . . . . . 12

Beaver Valley ... . . . . . . . . . . . . . 14

Big Rock Point ................. 16

Browns Ferry 1, 2, \& 3 ............... . . 18

Brunswlck 1 \& 2 . . . . . . . . . . . . . 20

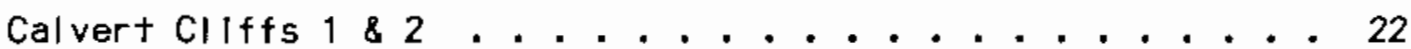

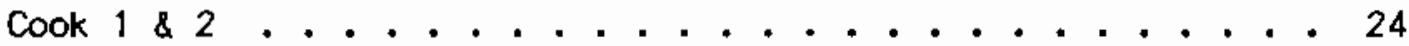

Cooper Station ........................ 26

Crystal River... . . . . . . . . . . . . . 28

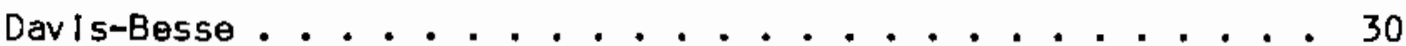

Dresden 1, 2, \& 3.................. . 32

Duane Arnold . . . . . . . . . . . . . . . . 34

J. M. Farley . . . . . . . . . . . . . . 36

J. A. Fitzpatrick ................. 38

Fort Calhoun .................... . . 40

R. E. Ginna ................... 42

Haddam Neck (Connecticut Yankee) . . . . . . . . . . . 44

Edwin 1. Hatch ................... 46 


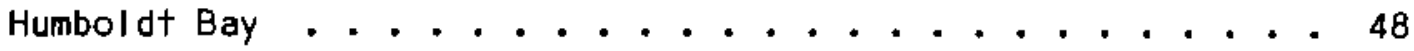

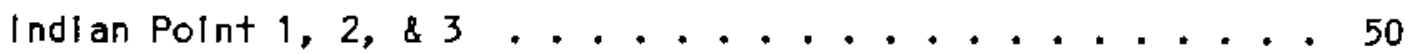

Kewaunee ...................... . . 52

La Crosse.. . . . . . . . . . . . . . . 54

Malne Yankee ................... 56

Mllistone Polnt 1 \& 2 ................. . . 58

Monticello ........................ 60 60

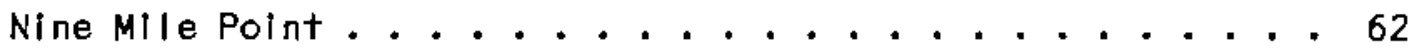

North Anna . . . . . . . . . . . . . . . 64

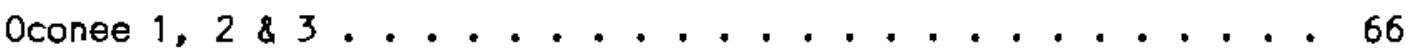

Oyster Creek ..................... 68

Pallsades . . . . . . . . . . . . . . 70

Peach Bottom $2 \& 3$ \& . . . . . . . . . . . . 72

Pligrim....................... . . 74

Point Beach 1 \& 2 . . . . . . . . . . . . . 76

Pralrie $\mid$ sland 1 \& 2 . . . . . . . . . . . . . . 78

Quad Citles 1 \& 2 . . . . . . . . . . . . . . 80

Rancho Seco.................... 82

H. B. Robinson . . . . . . . . . . . . . . . 84

St. Lucle . . . . . . . . . . . . . 86

Salen.......................... 88

San Onofre ....................... 90

Surry 1 \& 2 . . . . . . . . . . . . . . . 9 92

Three Mlle Island 1 \& 2 . . . . . . . . . . . . . . . 94 
Trojan ......................... 96

Turkey Polnt $3 \& 4$. . . . . . . . . . . . . . . . . 98

Vermont Yankee .......................... 100

Yankee Rowe. . . . . . . . . . . . . . . . . 102

Zion $1 \& 2$ \& . . . . . . . . . . . . . . 104

REFERENCES . . . . . . . . . . . . . . . . . 107

APPENDIX . . . . . . . . . . . . . . . . . . . A-1 



\section{INTRODUCTION}

The Pacific Northwest Laboratory (PNL) is operated by Battelle Memorial Institute for the Department of Energy (DOE). This study, sponsored by the Nuclear Regulatory Commission (NRC) and conducted at PNL, estimates radiation dose commitments from reported radionucl lde releases at 68 commercial power reactors operating during 1979. This work is a continuation of the study done for 1975 through 1978 releases (Baker, Soldat and watson 1977, Baker 1979 and 1980, and Peloquin et. al. 1982). In this study, as in those previous, we estimated the population (collective) dose commitment ${ }^{(a)}$ from both the liquid and gaseous releases to four age groups making up the population residing in the region of the site: infant 10 to $1 \mathrm{yr}$ ), chlld (1 to $11 \mathrm{yr}$ ), teen-ager ( 11 to $17 \mathrm{yr}$ ) and adult ( $17 \mathrm{yr}$ and older).

The particular organs of reference in this study are listed in Table 1. The major pathways by which radionuclides travel from the reactor to the individual receptors are shown in Table 2. Other posslble liquid pathways such as direct exposure from waterborne activities (swimming, boating, shoreline recreation) and internal exposure through ingestion of food produced using contaminated irrigation water were not included. This was because we have found from past experience that the doses from these pathways is generally much smaller than the doses from the pathways considered in this study.

The "source terms" used to estimate dose commitments produced from each slte were the annual measured releases of radloactive materlals as reported to the NRC by the plant operators and subsequently published in an NRC public document (TIchler and Benkovitz, 1981).

The regional population for which we estimated dose commitments included those persons estimated to be living in a reglon between 2 and 80 $\mathrm{km}$ around the reactor sltes during 1979. We estimated the numbers of persons by extrapolating Bureau of Census data for 1970 to 1979. Atmospheric transport factors (annual average dllution and annual average deposition) were calculated for the region around each site using approprlate meteorological data supplied by the NRC's Offlce of Nuclear Reactor Regulation. To calculate the doses, we used models approved by the NRC. We incorporated these models into two small computer codes to expedite the dose calculations involved for each site.

(a) As used in this report, dose commitment describes the total-body dose equivalent in rem (1 rem $=0.01$ sievert) recelved over 50 years from intake during the year in which radloactive materials were released into the environment from the plants. 
IABLE 1. Organs Considered in This Study

$\begin{array}{lc}\text { Organs Affected by } & \text { Organs Affected by } \\ \text { Alrberne Releases } & \text { Waterborne Releases } \\ \text { Total body } & \text { Total body } \\ \text { Thyrold } & \text { Thyrold } \\ \text { Bone } & \text { Bone } \\ \text { GI tract } & \text { Gl tract } \\ \text { Liver } & \text { Liver } \\ \text { Lung } & \end{array}$

IABLE 2. Pathways Considered in This Study by Which Radionuclides Travel from Reactors to Persons

Pathways for

Airborne Re leases

Air submersion

Contaminated ground

Inhalation

Ingestion of food crops

and animal products
Pathways for Waterborne Releases

Ingestion of drinking water Ingestion of $f i s h$ and invertebrates 
Site-specific parameters other than releases, meteorology and population were obtalned from environmental statements for the varlous reactors when avallable (Table 3). Such parameter values include the total population drinkling contaminated water, river flow, dilution flow from the reactors (for sltes not on rivers), $f$ ish and invertebrate harvest for reglon, and dilution factors for drinking water and aquatic foods.

The reactors included in this study, their type, licensed thermal power rating and net electrical output for 1979 are listed in Table 4. Those reactors whlch had an operating augmented gaseous radioactive waste system in 1979 are identifled in the table. Populations at risk and the dose commitments derived in the study are al so tabulated.

\section{Site-Dependent Parameters}

The section entitled Site Summarles gives the location (Including latitude and longltude) for each reactor site and the estimated 1979 population within 2 to $80 \mathrm{~km}$ around the site. This population is derived from the 1970 census by extrapolation to 1979. In addition, the location of major metropolitan centers within $80 \mathrm{~km}$ are listed along with their 1979 extrapolated populations. The populations of the Standard Metropolitan Statistical Areas (SMSA) are glven where applicable. Next, the sitespecific data pertinent to the airborne pathways are specifled. The average production rates of vegetable crops and animal products are given for the area ithin an $80-\mathrm{km}$ radius based upon the statewide average. This production has been reduced for sttes on lakes and seacoasts to account for the presence of the body of water. An animal grazing factor is estimated for each site location. This factor accounts for the fraction of the year during which grazing animals such as milk cows and beef cattle graze on fresh pasture in the reglon around the site. After average production rates are given, the period of record and the percent data recovery of the meteorological data used in calculating diffusion factors is indicated.

Varlous site-dependent factors associated with the waterborne pathways are presented next. For lake and ocean sites, we used the average dilution of plant effluents for the year 1979 specifled by Tlchler and Benkovitz (1981). For river sites, the average annual river flow is tabulated. This flow was used in place of a dilution flow from the plant to account for dilutions of liquid releases at the locations of probable intake of drinking water and aquatic food catch. Any exceptions to this scheme have been footnoted. Next is shown the estimated 1979 population utilizing drinking water drawn from supplles containing dlluted effluents from the site. These are shown with an estimated dilution factor where applicable. Fish and invertebrate catch data taken from the respective plant environmental statement, when avallable (see Table 3), are listed next, along with estimated dilution factors for the lake and ocean sites. 


\section{IABLE 3. Environmental Statements for Power Plants Included in this study}

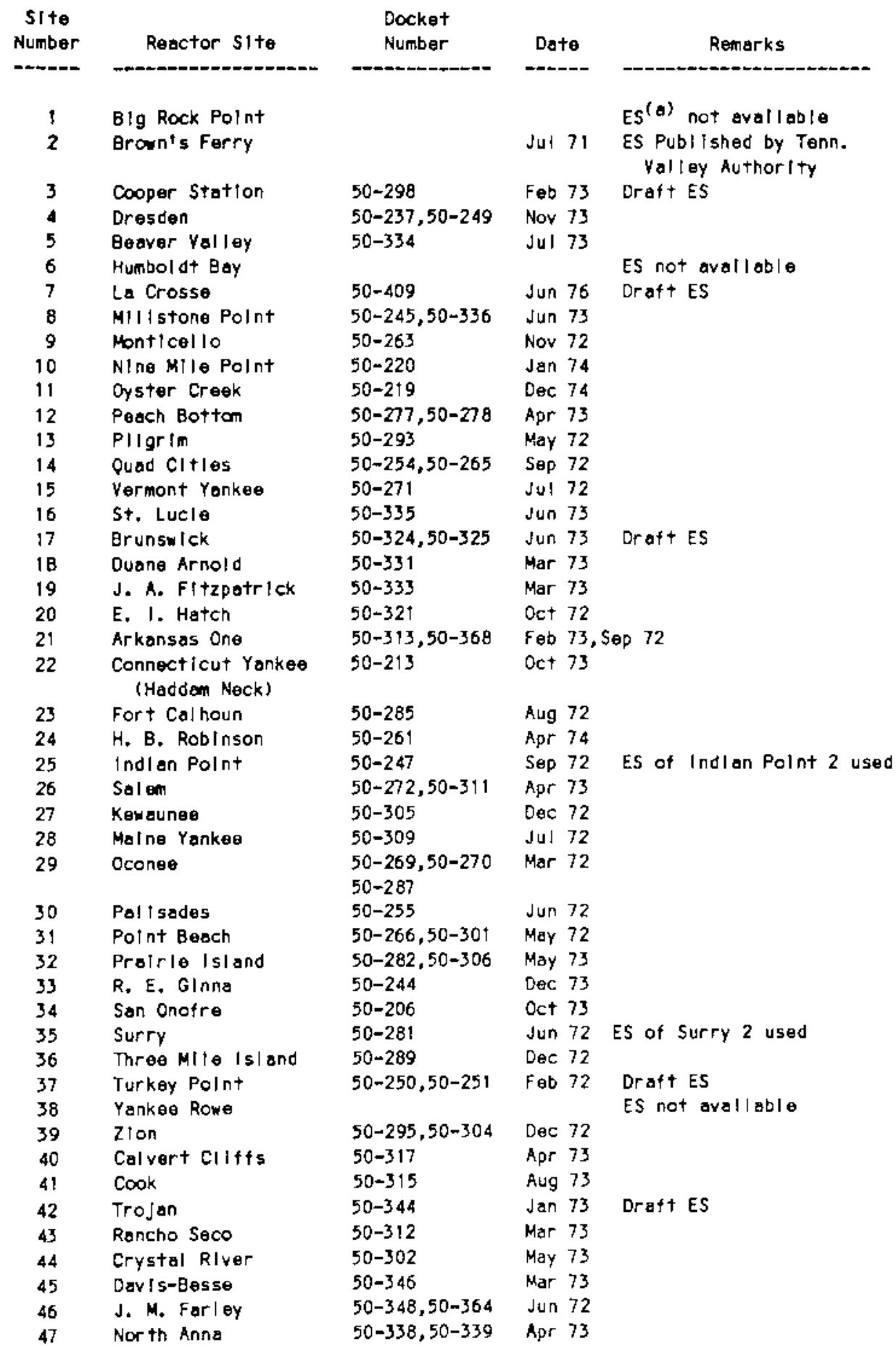

(a) Enviromental Statement 


\begin{tabular}{|c|c|c|c|c|c|c|c|c|c|c|}
\hline \multirow[b]{2}{*}{ site } & \multirow[b]{2}{*}{ Unit } & \multirow{2}{*}{ Type } & \multirow{2}{*}{$\begin{array}{l}\text { Lit censed } \\
\text { Thermat } \\
\text { Power } \\
\text { (itus) }\end{array}$} & \multirow{2}{*}{$\begin{array}{c}\text { Electr 1c } \\
\text { Enersy } \\
\text { Generatlon } \\
1979 \\
(T w, h r)(s)\end{array}$} & \multirow{2}{*}{ 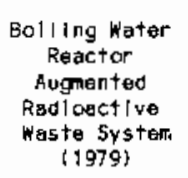 } & \multicolumn{3}{|c|}{$\begin{array}{l}\text { Popul otlion } \\
\text { Dose Comm I tment } \\
\text { (Person-ren) }\end{array}$} & \multirow{2}{*}{$\begin{array}{l}\text { Population } \\
\text { At Risk }\end{array}$} & \multirow{2}{*}{$\begin{array}{c}\text { Average Individual } \\
\text { Total-Body Dose } \\
\text { Comm itment } \\
\text { (mrems) }\end{array}$} \\
\hline & & & & & & Liquid & Alr & Total & & \\
\hline $\begin{array}{l}\text { Arkansas One } \\
\text { Arksnsas One }\end{array}$ & $z_{\text {TOTAL }}^{3}$ & $P_{R}$ & $\begin{array}{l}2568 \\
2815 \\
5383\end{array}$ & $\begin{array}{l}3.32 \\
0.88 \\
4.20\end{array}$ & $=$ & 0.35 & 0.12 & 0.47 & $1.7 \mathrm{ES}$ & $2.8 \mathrm{E}-3$ \\
\hline Beaver valley & 1 & PrR & 2652 & 1.79 & - & 0.012 & 0.16 & 0.17 & $3.7 E 6$ & $4.6 \mathrm{E}-5$ \\
\hline BIg Rock Polnt & 1 & $B W R^{(c)}$ & 240 & 0.114 & NO & 8.9 & 0.12 & 9.0 & $1.3 E 5$ & $6.9 \mathrm{E}-2$ \\
\hline $\begin{array}{l}\text { Browns Ferry } \\
\text { Erowns Ferry }\end{array}$ & $\begin{array}{l}\frac{1}{2} \\
\frac{2}{3} \\
\text { TOTAL }\end{array}$ & $\begin{array}{l}\text { BWR } \\
\text { QWWR } \\
\text { BWWR } \\
\text { LWW }\end{array}$ & $\begin{array}{l}3293 \\
3293 \\
393 \\
9879\end{array}$ & $\begin{array}{l}- \text { NA- } \\
\text {-NA- } \\
\text {-NA- } \\
20.40\end{array}$ & $\begin{array}{l}\text { YES } \\
\text { YES } \\
\text { YES }\end{array}$ & 3.3 & 6.3 & 9.6 & 6.8E5 & $1.4 E-2$ \\
\hline $\begin{array}{l}\text { Brunswlek } \\
\text { Brunswlck }\end{array}$ & $2_{\text {TOTAL }}^{1}$ & $\begin{array}{l}\text { BWwR } \\
\text { EWHR }\end{array}$ & $\begin{array}{l}2436 \\
2436 \\
4872\end{array}$ & $\begin{array}{l}\text {-NA- } \\
\text { NAA- } \\
6.82\end{array}$ & $\begin{array}{l}\text { NO } \\
\text { NO }\end{array}$ & 0.020 & 2.2 & 2.2 & $1.9 \mathrm{E} 5$ & $1.2 \mathrm{E}-2$ \\
\hline $\begin{array}{l}\text { Calvert Clifts } \\
\text { Calvert Clifts }\end{array}$ & $2^{1}$ & $\underset{P}{P} \mathbb{R} R$ & $\begin{array}{l}2700 \\
27700 \\
5400\end{array}$ & $\begin{array}{l}-\mathrm{NA-} \\
-\mathrm{NAL} \\
\mathrm{N} .68\end{array}$ & $=$ & 2.1 & 0.92 & 3.0 & $2.4 E 6$ & $1.2 E-3$ \\
\hline $\begin{array}{l}\text { cook } \\
\text { cook }\end{array}$ & $2_{\text {TOTAL }}^{1}$ & $\underset{R}{P} \mathbb{R}$ & $\begin{array}{l}3250 \\
3391 \\
6641\end{array}$ & $\begin{array}{l}-\mathrm{NA}- \\
-\mathrm{NAA} \\
11.60\end{array}$ & 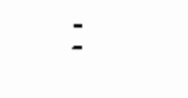 & 17 & 0.046 & 17 & $1.1 E 6$ & $1.5 \mathrm{E}-2$ \\
\hline Cooper station & 1 & EWR & 2381 & 4.99 & YES & 0.0073 & 0.34 & 0.35 & $1.8 \mathrm{ES}$ & $1.9 E-3$ \\
\hline Crystal River & 3 & 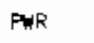 & 2452 & 3.76 & - & 0.40 & 0.25 & 0.65 & $2.3 E 5$ & $2.8 E-3$ \\
\hline $\operatorname{Dav} 15$-Besse & 1 & PNR & 2772 & 3.13 & - & 0.51 & 0.013 & 0.52 & $1.8 E 6$ & $3.0 E-4$ \\
\hline $\begin{array}{l}\text { Dresden } \\
\text { Dresten }\end{array}$ & $\begin{array}{l}\frac{1}{2} \\
\frac{3}{3} \text { TOTAL }\end{array}$ & $\begin{array}{l}\text { BWR } \\
\text { BWR } \\
\text { BWhR } \\
L\end{array}$ & $\begin{array}{l}700 \\
2527 \\
2527 \\
5754\end{array}$ & $\begin{array}{l}0 \\
8.42 \\
8.42\end{array}$ & $\begin{array}{l}\text { NO } \\
\text { NO } \\
\text { NO }\end{array}$ & 0 & 15 & 15 & $6.4 E 6$ & $2.4 E-3$ \\
\hline Duane Arnold & 1 & BWR & 1658 & 2.90 & YES & 0.059 & 0.48 & 0.54 & $5.7 E 5$ & $9.4 \mathrm{E}-4$ \\
\hline J. M. Farley & $t$ & $P w R$ & 2652 & 1.74 & - & 0.0025 & 0.19 & 0.19 & $3.3 \mathrm{ES}$ & $5.8 \mathrm{E}-4$ \\
\hline J. A. Fitzpgtrick & 1 & BWR & 2436 & 2.96 & YES & 0.18 & 0.060 & 0.24 & $8.1 E 5$ & $3.0 \mathrm{E}-4$ \\
\hline Fort Cal houn & 1 & PXR & 1420 & 3.67 & - & 0.38 & 0.0072 & 0.39 & $7.5 E 5$ & $5.1 E-4$ \\
\hline R. E. GIIna & 1 & $P \times R$ & 1520 & 2.96 & - & 0.071 & 0.035 & 0.11 & $1.1 E 6$ & $9.4 \mathrm{E}-5$ \\
\hline Haddam Neck & 1 & PXR & 1825 & 4.12 & - & 0.22 & 3.4 & 3.6 & $3.4 E 6$ & $1 . t E-3$ \\
\hline E. 1. Hatch & 1 & BWR & 2436 & 5.10 & YES & 0.12 & 0.012 & 0.13 & $2.8 E 5$ & $4.7 \mathrm{E}-4$ \\
\hline Hunbol d+ Bay & 1 & BWR & 220 & 0 & no & $1.1 \mathrm{E}-4$ & $5.7 E-5$ & $1.6 E-4$ & $4 \quad 1.155$ & $1.5 E-6$ \\
\hline $\begin{array}{l}\text { Indian Polnt } \\
\text { Indian Potnt }\end{array}$ & $\begin{array}{l}\frac{1}{2} \\
\frac{2}{3} \text { TOTAL }\end{array}$ & 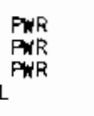 & $\begin{array}{r}615 \\
2758 \\
2760 \\
6133\end{array}$ & $\begin{array}{l}4.80 \\
4.79 \\
9.59\end{array}$ & 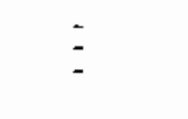 & 0.48 & 5.4 & 5.9 & 1.687 & $3.7 E^{-4}$ \\
\hline Keraunes & 1 & PArF & 1650 & 3.44 & - & 1.8 & 0.0034 & 1.8 & 0.115 & $3.0 \mathrm{E}-3$ \\
\hline La Crosse & 1 & BWR & 165 & 0.20 & No & 3.7 & 0.44 & 4.1 & $3.4 E 5$ & $1.2 \mathrm{E}-2$ \\
\hline Maine Yankee & 1 & PxR & 2440 & 4.54 & - & 8. $1 E-4$ & 0.041 & 0.042 & $5.7 E 5$ & $7.3 \mathrm{E}-5$ \\
\hline $\begin{array}{l}\text { Mfllstone Polnt } \\
\text { Mllistone Polnt }\end{array}$ & $2_{\text {TOTAL }}^{1}$ & ${ }_{L}^{B W R}$ & $\begin{array}{l}2011 \\
2550 \\
4571\end{array}$ & $\begin{array}{l}4.22 \\
4.36 \\
8.58\end{array}$ & No & 0.0051 & 1.8 & 1.8 & $2.5 E 6$ & $7,2 \mathrm{E}-4$ \\
\hline$m o n+1<e 110$ & 1 & BWR & 1670 & 4.40 & YES & 0 & 0.18 & 0.18 & $2.1 \mathrm{E} 6$ & $8.5 E-5$ \\
\hline Ning Mlle Polnt & 1 & BWR & 1850 & 3.00 & no & 140 & 0.082 & 140 & $8.1 E 5$ & $1.7 \mathrm{E}-1$ \\
\hline North Anne & 1 & $\mathrm{AWR}$ & 2775 & 4.19 & - & 4.8 & 0.055 & 4.9 & $9.6 E 5$ & $5.1 E-3$ \\
\hline $\begin{array}{l}\text { Dconee } \\
\text { Oconee }\end{array}$ & $\begin{array}{l}1 \\
\frac{2}{3} \\
\text { TOTAL }\end{array}$ & L & $\begin{array}{l}2568 \\
2568 \\
2568 \\
7704\end{array}$ & $\begin{array}{l}-N A- \\
-N A- \\
-N A- \\
13.20\end{array}$ & $\bar{z}$ & 5.0 & 0.79 & 5.8 & $7.5 \mathrm{ES}$ & $7.7 \mathrm{E}-3$ \\
\hline Oyster Creek & 1 & BWR & 1930 & 4.56 & no & $1.4 E-4$ & 220 & 220 & $3.3 \mathrm{E} 6$ & $6.6 \mathrm{E}-2$ \\
\hline Pal I sades & 1 & PrR & 2530 & 3.43 & - & 0.12 & 0.0033 & 0.12 & $1.0 \mathrm{E} 6$ & $1.2 \mathrm{E}-4$ \\
\hline $\begin{array}{l}\text { Peach Bottom } \\
\text { Peach Bottom }\end{array}$ & $3_{\text {TOTAL }}^{2}$ & $\begin{array}{l}\text { BWR } \\
\text { BWR } \\
\end{array}$ & $\begin{array}{l}3293 \\
3293 \\
6586\end{array}$ & 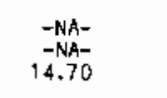 & $\begin{array}{l}\text { YES } \\
\text { YES }\end{array}$ & 16 & 14 & 30 & 4.1E6 & $7.4 E-3$ \\
\hline Pilgrim & 1 & EWR & 1998 & 4.84 & YES & 0.024 & 3.1 & 3.1 & 4.3E6 & $7.2 \mathrm{E}-4$ \\
\hline $\begin{array}{l}\text { Point Beach } \\
\text { Point Eeach }\end{array}$ & $\frac{1}{2}_{\text {TOTAL }}$ & $\stackrel{P}{P}$ & $\begin{array}{l}1518 \\
1518 \\
3036\end{array}$ & $\begin{array}{l}-\mathrm{NA-} \\
\text {-NA- } \\
6.77\end{array}$ & $=$ & 0.055 & 0.20 & 0.25 & $6.0 \mathrm{E} 5$ & $4.2 E-4$ \\
\hline $\begin{array}{l}\text { Prairis Island } \\
\text { Pralrie 1sland }\end{array}$ & $\stackrel{1}{2}_{\text {TOTAL }}$ & $\stackrel{P W R}{P}$ & $\begin{array}{l}1650 \\
1650 \\
3300\end{array}$ & $\begin{array}{l}\text {-NA- } \\
\text {-NA- } \\
7.10\end{array}$ & $=$ & 0.0033 & 0.10 & 0.10 & $2.2 E 6$ & $4.7 E-5$ \\
\hline $\begin{array}{l}\text { Quad Clties } \\
\text { Quad } \mathrm{Cl}+1 \text { as }\end{array}$ & $i_{\text {TOTAL }}^{1}$ & 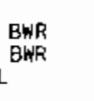 & $\begin{array}{l}2511 \\
2511 \\
5022\end{array}$ & $\begin{array}{l}-N A- \\
\text {-NA- } \\
B .76\end{array}$ & $\begin{array}{l}\text { YES } \\
\text { YES }\end{array}$ & 2.5 & 4.0 & 6.5 & $6.7 E 5$ & $9.7 \mathrm{E}-3$ \\
\hline Rancho Seco & 1 & PuR & 2772 & 5.71 & - & 0 & 0.32 & 0.32 & $1.6 E 6$ & $2.1 \mathrm{E}-4$ \\
\hline H. B. Robinson & 2 & FrR & 2200 & 4.00 & - & 0.17 & 0.024 & 0.19 & $6.5 E 5$ & $3.0 \mathrm{E}-4$ \\
\hline St. Luele & 1 & $P x R$ & 2560 & 4.88 & - & 0.15 & 0.61 & 0.76 & 3.055 & $2.5 E-3$ \\
\hline Salem & 1 & PWR & 3338 & 2.04 & - & 0.23 & 2.1 & 2.3 & A.9E6 & $4.8 E-4$ \\
\hline San Onotre & 1 & $P$ R & 1347 & 3.36 & - & 0.49 & 0.031 & 0.52 & $4.0 E 6$ & $1.3 \mathrm{E}-4$ \\
\hline $\begin{array}{l}\text { Surry } \\
\text { Surry }\end{array}$ & $2_{\text {TOTAL }}^{1}$ & $L_{R}$ & $\begin{array}{l}2441 \\
2441 \\
4882\end{array}$ & $\begin{array}{l}\text { NA- } \\
\text {-NA } \\
2.87\end{array}$ & $=$ & 0.92 & 0.12 & 1.0 & $1.8 \mathrm{EG}$ & $5.9 E-4$ \\
\hline $\begin{array}{l}\text { Three Mile Isiano } \\
\text { Three Mile is and }\end{array}$ & $\frac{1}{2}$ & $\begin{array}{l}P W R \\
P \mathscr{R} R \\
P\end{array}$ & $\begin{array}{l}2535 \\
2772 \\
5307\end{array}$ & $\begin{array}{l}0.85 \\
\begin{array}{l}\text { O. NA- } \\
\text { NA- }\end{array}\end{array}$ & $=$ & 0.291 & 1300 & 1300 & $1.8 \mathrm{E} 6$ & $7.2 \mathrm{E}-1$ \\
\hline Trojar: & 1 & $\mathrm{PWR}$ & 3411 & 5.27 & - & 0.0062 & 0.042 & 0.048 & $1.4 E 6$ & $3.5 E-5$ \\
\hline $\begin{array}{l}\text { Turkey Point } \\
\text { Turkey Polnt }\end{array}$ & $\begin{array}{l}3 \\
4 \\
\text { TOTAL }\end{array}$ & $\underset{P \rightarrow R}{\operatorname{Par} R}$ & $\begin{array}{l}2200 \\
2200 \\
4400\end{array}$ & $\begin{array}{l}-\mathrm{NAA}- \\
-\mathrm{NAB} \\
6.71\end{array}$ & : & 0.0040 & 0.17 & 0.17 & $2.2 E 6$ & $8.1 E-5$ \\
\hline Yermont Yarkee & 1 & GWR & 1593 & 3.45 & YES & 0.0021 & 0.46 & 0.46 & $1.4 \mathrm{E} 6$ & $3.3 \mathrm{E}-4$ \\
\hline Yankee Rowe & 1 & $\mathrm{PWR}$ & 600 & 1.23 & - & 0.38 & 0.018 & 0.40 & $1.6 E 6$ & $2.6 \mathrm{E}-4$ \\
\hline zion & ${ }^{1}$ & $\underset{P W R}{P W R}$ & $\begin{array}{l}3250 \\
3350 \\
6500\end{array}$ & $\begin{array}{c}-\mathrm{NA-} \\
\begin{array}{c}\mathrm{NA-} \\
10.30\end{array}\end{array}$ & $=$ & 11 & 3.5 & 14 & $7.0 E 6$ & $2.1 E-3$ \\
\hline TOTAL FOR ALL SITES & & & & 250 & & 220 & 1600 & 1800 & $9.4 E 7$ & --- \\
\hline Ar lthmetlc Mean & & & & 5.3 & & 4.7 & 34 & 38 & $2.0 E 6$ & $2.4 \mathrm{E}-2$ \\
\hline Geometr ic Mean & & & & 3.9 & & 0.18 & 0.26 & 1.1 & $1.1 E 6$ & $1.0 \mathrm{E}-3$ \\
\hline
\end{tabular}

(a) 1 Th.tr $=3.6 \mathrm{Et} 5$ joules

(b) Pressurized water reactor

(c) Eolling water reactor

-NA- = Not avallable 

This report consists of a summary of values used for site-specific parameters at each site, as explained above, and the results of population dose commitment calculations. The population dose commitments are presented in two tables facing the page summarlzing site-specific parameters for that site. These tables include both liquid and alrborne pathway dose commltments for the several organs of reference for each age group investigated. They also include the dose to the whole population which includes all age groups. The airborne population dose commitments for each of 160 segments partitioning the reglon around the slte ${ }^{(a)}$ were divided by the population residing with in that segment to derive an average individual dose for that segment. These doses are summarized as a histogram showing percent of the population recelving a given dose level for each site. The fractional population dose from the liquid pathway was not determined in this manner, because the NRC does not at present take into account the location of individuals exposed via this pathway, except those exposed through ingestion of drinking water.

Population dose commitments estimated for both the I lquid pathways and airborne pathways varled idely over the 47 sltes (68 reactors) studied. The total dose commltments (from both pathways) varied from a high of 1300 to a low of 0.0002 person-rem. The arithmetlc mean for the dose from I lquid pathways was 5 person-rem and the mean for the dose from airborne pathways was 34 person-rem (see Table 4).

Releases from Monticello, Rancho Seco, and Dresden resulted in the smallest doses from I iquid pathways (zero to near zero, respectively). This was because no liquid releases were reported for Monticello and Rancho Seco, and the recelving waters for the Dresden site are contaminated with Chicago sewage to such an extent as to severely limit use of this water for drinking or fishing. The largest I lquid-pathway dose to the total body was 140 person-rem at Nine Mile Polnt. This dose resulted primarlly from the radloactive ceslum ( $\mathrm{Cs}-134$ and $\left.\mathrm{Cs}_{5} 137\right)$ released by this plant.

The lowest total alrborne pathway dose to the total body was estimated for Humboldt Bay $(0.0001$ person-rem); and Kewaunee and Pal isades 10.003 person-rem) each. The highest doses were at Three Mile lsland $(1300$ person-rem) and Oyster Creek (200 person-rem). The large value for Three Mile $|s|$ and includes the releases of TMI-2 during the accident in the spring. The major contributors to these doses were the noble gases: $\mathrm{Xe}-133$ and $x_{e}-135$. The total population dose commitments from all sites for 1979 were estimated to be 220 person-rem via I lquid pathways and 1600 person-rem via the alrborne pathways (Table 4).

(a) See Appendix for definition of segments. 
Compared to 1978, the "l lquld dose" is higher (220 vs. 110); and al so, the "alr dose" is higher (1600 vs. 530). Table 5 compares the total population dose commitments estimated for the past five years.

Figure 1 shows graphically the wide range of the alrborne population dose commitments for the reactor sites. The median, upper and lower quartiles and upper and lower octlles for the distribution of doses calculated for each of 160 segments are Indlcated for each site. Figure 2 is a histogram for all 47 sites taken together. We can see from this plot that about $25 \%$ of the total population at risk $(94 \mathrm{mill}$ lon) would each recelve a dose commitment of between 0.0003 and 0.001 mrem. We can see further that $<0.1 \%$ recelve a dose which is less than $1 \times 10^{-6}$ mrem. Although not discernible from the plot, $0.07 \%$ received a dose of between 3 and 10 mrem. and $0.009 \%$ or about 9,000 persons recelved average dose commitments greater than $10 \mathrm{mrem}$. The sites responsible for the alrborne average dose commitments greater than $10 \mathrm{mrem}$ were Three Mile Island and Oyster Creek. However, no attempt was made in this study to estimate the maximum dose commitment recelved by any one individual from the radionuclides released at any of the sites.

We should polnt out here, however, that the doses estimated in this study are extremely low compared to an average annual background dose of approximately $100 \mathrm{mrem}$. We have compared dose commitments calculated in this study with annual background, however this comparlson is not quite exact, since these dose comm 1 tments are those total-body doses recelved from the year's (1979) effluent release, over 50 years of a person's Ilfetime. However, most of the dose commitment calculated here is delivered in the first year, so the comparison is reasonably valld.

For comparison purposes the doses in the site summary tables are given to two signiflcant figures; however, the data and models used in their calculation ilmit their accuracy to at most one significant figure.

IABLE 5. Comparison of Annual Population Oose Commitments for the Last Five Years (person-rem)

$\begin{array}{lrrrrr} & 1975 & 1976 & 1977 & 1978 & 1979 \\ \text { Liquid } & 76 & 82 & 160 & 110 & 220 \\ \text { Air } & 1300 & \frac{390}{470} & \frac{540}{700} & \frac{530}{640} & \frac{1600^{(a)}}{1800} \\ \text { TOTAL } & 1400 & & 470\end{array}$

(a) Primarily due to the TMI-2 accident. 


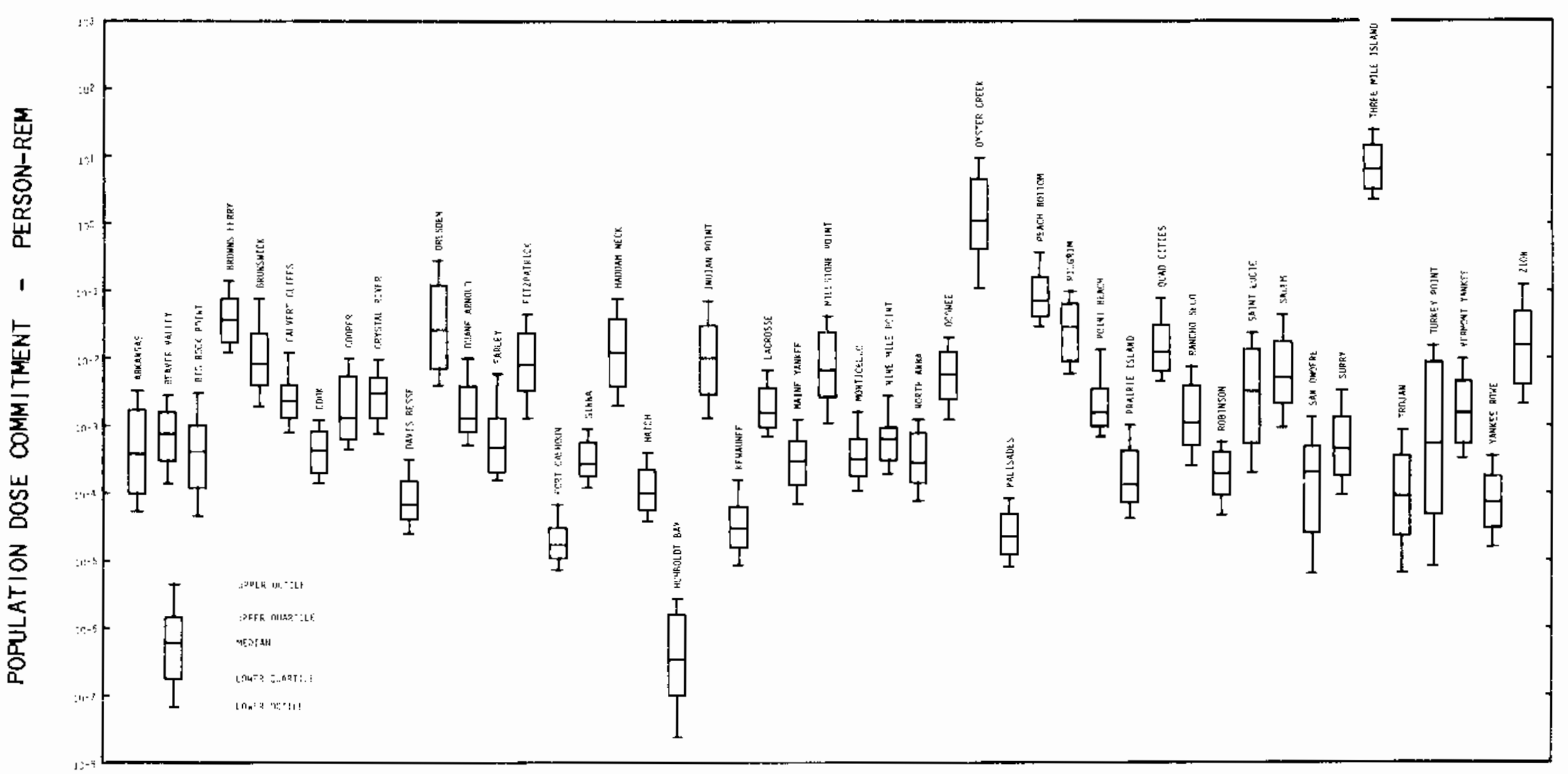

FIGURE 1. Alrborne Population Dose Commitments for the Reactor Sites, 1979 


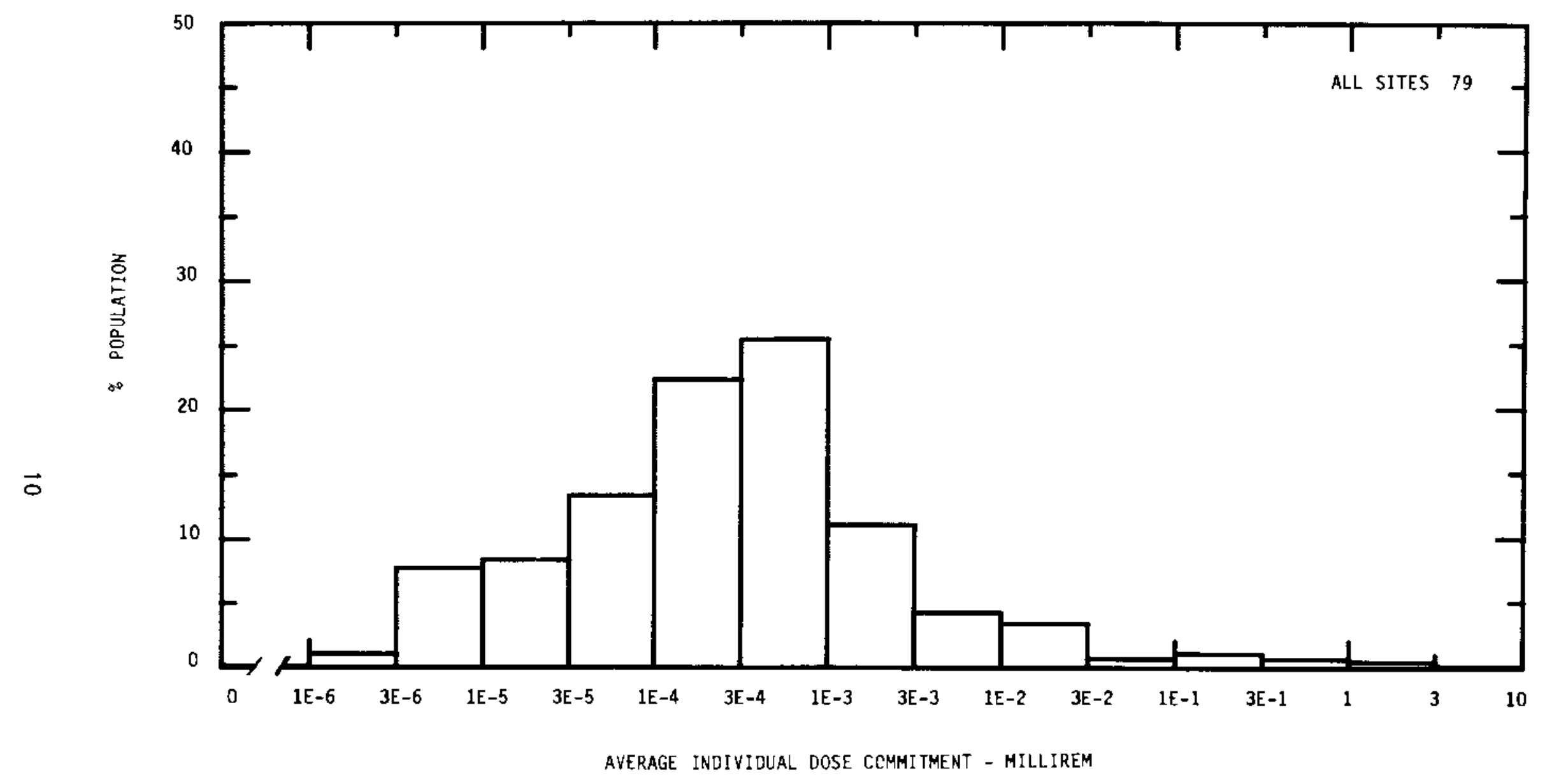

FIGURE 2. Fraction of Total Population Receiving Varlous Individual Dose Comitments for AlI Sites, 1979 
SUIE SUMMARJES

1979 
SIte: ARKANSAS ONE

Lecation:

N $35.3075^{\circ}$

POPE COUNTY, ARKANSAS

$93.2308^{\circ}$

Total Population within 20-to-80-km Region: 1.7E5

Major Metropolitan Centers within Region:

Center

Russel Iville

Convay
Population

13,000

18,000
Lecation

$10 \mathrm{~km} \quad \mathrm{E}$

$77 \mathrm{~km} \quad$ ESE

SITE SPECIFIC DATA - AIRBORNE PATHWAYS

Average Annual State Production

of Crops and Animal Products

In $80-\mathrm{km}$ Radius Circle

Reglonal Productivity Factor:

Animal Grazing Factor:

Meteorology Period of Record: 1 JAN 75 - 31 DEC 75 Recovery: 97\$

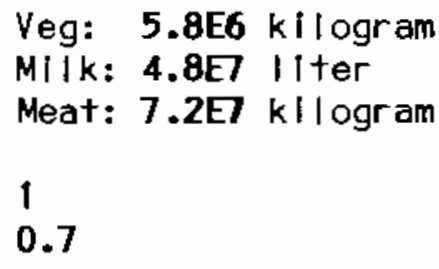

Veg: $5.8 E 6$ kilogram

SITE SPECIFIC DATA - WATERBORNE PATHWAYS vIa ARKANSAS RIVER

$\begin{array}{ll} & \text { Average River } \mathrm{Flow} \\ & \text { at Site: } 36,000 \mathrm{ft}^{3} / \mathrm{s} \\ \text { Drinking Water: } & \text { Exposed Population: None } \\ \text { Fish: } & \text { Edible Harvest: } 1.4^{(a)} \mathrm{kg} / \mathrm{yr} \\ & \text { Dilution Factor: } 1\end{array}$

Average River Flow

Exposed Population: None

Dilution Factor: 1 


\section{POPULATION DOSE-COMMITMENT ESTIMATES AND AVERAGE INDIVIDUAL DOSE-COMMITMENT HISTOGRAM FOR \\ ARKANSAS ONE 1 ND 2}

Dose Commitments (person-rem) from Liquid Pathways

Iotal Body

GL-LLI

Thyroid

Bene

Llver

Infant

$0.0 \mathrm{E}+00$

$0.0 E+00$

$0.0 E+00$

$0.0 \mathrm{E}+00$

$0.0 \mathrm{E}+00$

Child

$1.5 \mathrm{E}-02$

1. $2 \mathrm{E}-02$

1.1 E-02

$7.3 \mathrm{E}-02$

8. $4 \mathrm{E}-02$

Teen

Adult

2.9E -02

2. 5E-02

4. $4 \mathrm{E}-02$

$7.2 \mathrm{E}-02$

3. $1 \mathrm{E}-01$

2. 2E-01

5.1E-02

2.5E-01

4. 3E-01

TOTAL

3.5E-01

2.6E-01

$7.0 \mathrm{E}-02$

3.7E-01

5.8E-01

Dose Commitments (person-rem) from Airborne Pathways

\section{Iotal Body Gi-Ll Ihyrold Bone Llver Lung}

$\begin{array}{lllllll}\text { Infant } & 1.8 \mathrm{E}-03 & 1.8 \mathrm{E}-03 & 3.0 \mathrm{E}-03 & 1.8 \mathrm{E}-03 & 1.8 \mathrm{E}-03 & 1.9 \mathrm{E}-03 \\ \text { ChIId } & 2.0 \mathrm{E}-02 & 2.0 \mathrm{E}-02 & 2.7 \mathrm{E}-02 & 2.0 \mathrm{E}-02 & 2.0 \mathrm{E}-02 & 2.1 \mathrm{E}-02 \\ \text { Teen } & 1.4 \mathrm{E}-02 & 1.4 \mathrm{E}-02 & 1.7 \mathrm{E}-02 & 1.4 \mathrm{E}-02 & 1.4 \mathrm{E}-02 & 1.6 \mathrm{E}-02 \\ \text { Adult } & 8.7 \mathrm{E}-02 & 8.7 \mathrm{E}-02 & 9.8 \mathrm{E}-02 & 8.7 \mathrm{E}-02 & 8.7 \mathrm{E}-02 & 9.4 \mathrm{E}-02 \\ \text { TOTAL } & 1.2 \mathrm{E}-01 & 1.2 \mathrm{E}-01 & 1.5 \mathrm{E}-01 & 1.2 \mathrm{E}-01 & 1.2 \mathrm{E}-01 & 1.3 \mathrm{E}-01\end{array}$

Production/Consumption factors: (a)

Produce: <1 Milk: $2.2 \quad$ Meat: 5.4

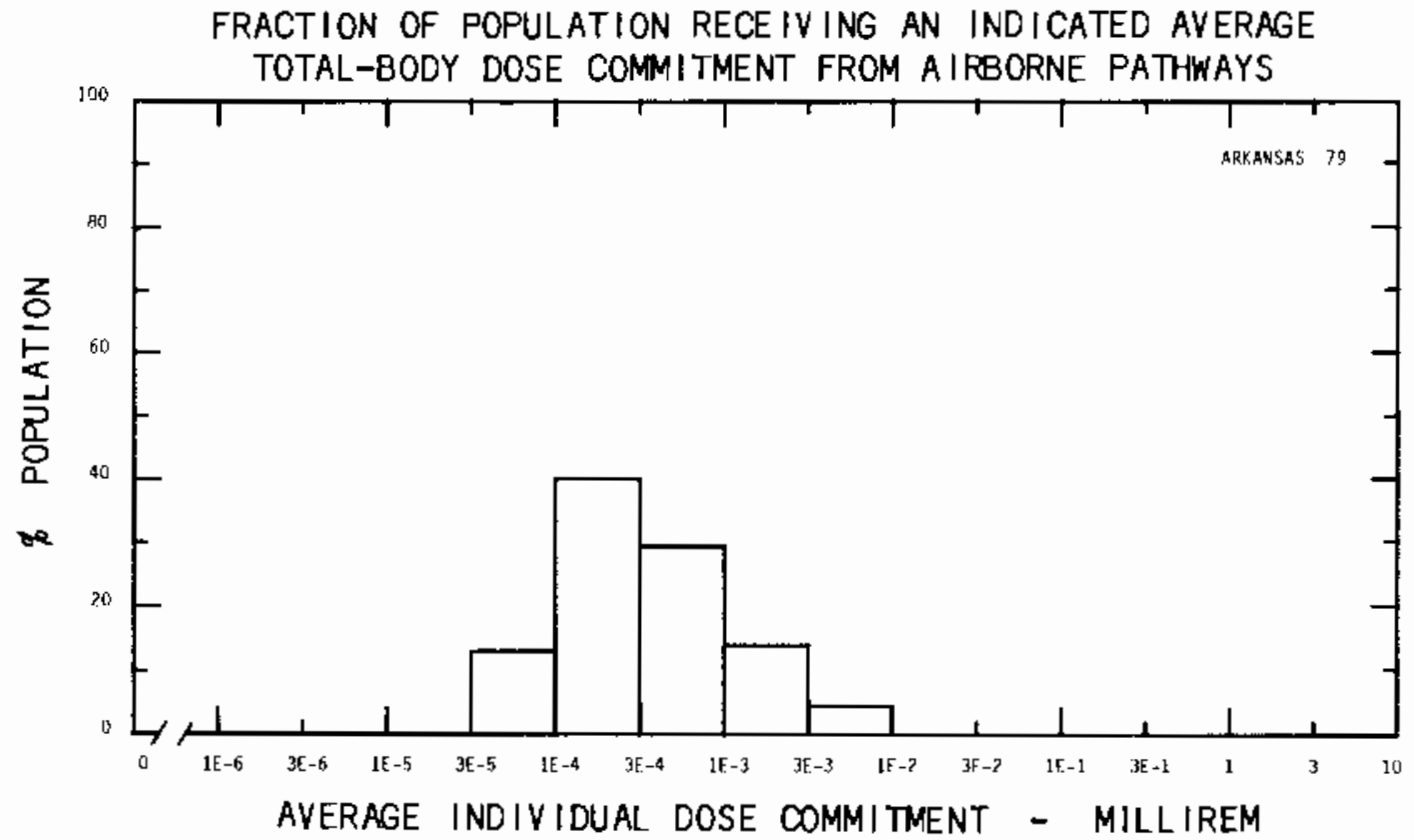

(a) See Appendix A, Page A-5, for explanation of this ratio. 
Site; BEAYER VALLEY

Lecation:

N $\quad 40.6214^{\circ}$
SHIPPINGPORT, PENNSYLYNIA

W $80.4347^{\circ}$

POPULATION DATA

Total Population within 20-to-80-km Reglon: 3.7E6

Major Metropolitan Centers Within Region:

Center

PIttsburgh Silsh

Youngstowiliarren Sish

Stuebenville

Wheel Ing
Population

\subsection{E6}

530,000

31,000

48,000
Lecation

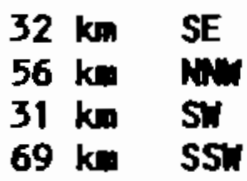

SITE SPECIFIC DATA - AIRBORNE PATHWAYS

Average Annual State Production

Of Crops and AnImal Products

In 80-km Radius Circle

Reglonal Productivity Factor:

Animal GrazIng Factor:
Veg: $5.3 E 7$ kilogram

MIIk: 5.3E8 I Iter

Meat: $5.4 E 7$ kilogram

1

0.5

Meteorology Perlod of Record: 1 JAN 77 - 31 DEC 77 Recovery: 92,

SITE SPECIFIC DATA - WATERBORNE PATHWAYS VIO OHIO RIVER

Average River Flow

at Site: $30,000 \mathrm{ft}^{3} / \mathrm{s}$

Drinking Water:

Exposed Population: 6,400

Dilution Factor: $12(\mathrm{a})$

FIsh:

Edible Harvest: $410 \mathrm{~kg} / \mathrm{yr}$

Dilution Factor: 1

(a) This factor accounts for the incomplete dilution of plant effluent by river at point of drinking water intake at Midiand. 
POPULATION DOSE-COMMITMENT ESTIMATES AND
AVERAGE INDIVIDUAL DOSE-COMMITMENT HISTOGRAM FOR

\section{BEAVER VALLEY}

Dose Commitments (person-rem) from Liquid Pathways

Total Body

Gl-LلL

Ihyreld

Bone

Luver

Infant

Child

Teen

Adult

TOTAL

$\begin{array}{ll}2.2 \mathrm{E}-04 & 2.2 \mathrm{E}-04 \\ 3.5 \mathrm{E}-03 & 2.6 \mathrm{E}-03 \\ 9.5 \mathrm{E}-04 & 1.1 \mathrm{E}-03 \\ 8.1 \mathrm{E}-03 & 1.1 \mathrm{E}-02 \\ 1.2 \mathrm{E}-02 & 1.4 \mathrm{E}-02\end{array}$

2.9E-04 1.8E-05

2.3E-04

2.9E-03 2.2E-04

2.5E-03

$1.1 \mathrm{E}-03 \quad 6.5 \mathrm{E}-05$

9.6E-04

8.8E-03 4.5E-04

$8.0 \mathrm{E}-03$

1.2E-02

1.3E-02

$7.6 \mathrm{E}-04$

$1.2 \mathrm{E}-02$

Dose Commitments (person-rem) from Alrborne Pathways

Iotal Body Gl-Lلl Ihyrold Bone Liver Lung

$\begin{array}{lllllll}\text { Infant } & 2.5 \mathrm{E}-03 & 2.5 \mathrm{E}-03 & 3.3 \mathrm{E}-03 & 1.8 \mathrm{E}-03 & 2.5 \mathrm{E}-03 & 2.7 \mathrm{E}-03 \\ \text { ChIId } & 2.8 \mathrm{E}-02 & 2.8 \mathrm{E}-02 & 3.2 \mathrm{E}-02 & 2.0 \mathrm{E}-02 & 2.8 \mathrm{E}-02 & 3.1 \mathrm{E}-02 \\ \text { Teen } & 1.9 \mathrm{E}-02 & 1.9 \mathrm{E}-02 & 2.1 \mathrm{E}-02 & 1.5 \mathrm{E}-02 & 1.9 \mathrm{E}-02 & 2.4 \mathrm{E}-02 \\ \text { Adult } & 1.1 \mathrm{E}-01 & 1.1 \mathrm{E}-01 & 1.2 \mathrm{E}-01 & 8.8 \mathrm{E}-02 & 1.1 \mathrm{E}-01 & 1.3 \mathrm{E}-01 \\ \text { TOTAL } & 1.6 \mathrm{E}-01 & 1.6 \mathrm{E}-01 & 1.8 \mathrm{E}-01 & 1.2 \mathrm{E}-01 & 1.6 \mathrm{E}-01 & 1.8 \mathrm{E}-01\end{array}$

Production/Consumption factors:

Produce: <1 Milk: 1.1 Meat: <1

FRACTION OF POPULATION RECEIVING AN INDICATED AVERAGE TOTAL-BODY DOSE COMMITMENT FROM AIRBORNE PATHWAYS

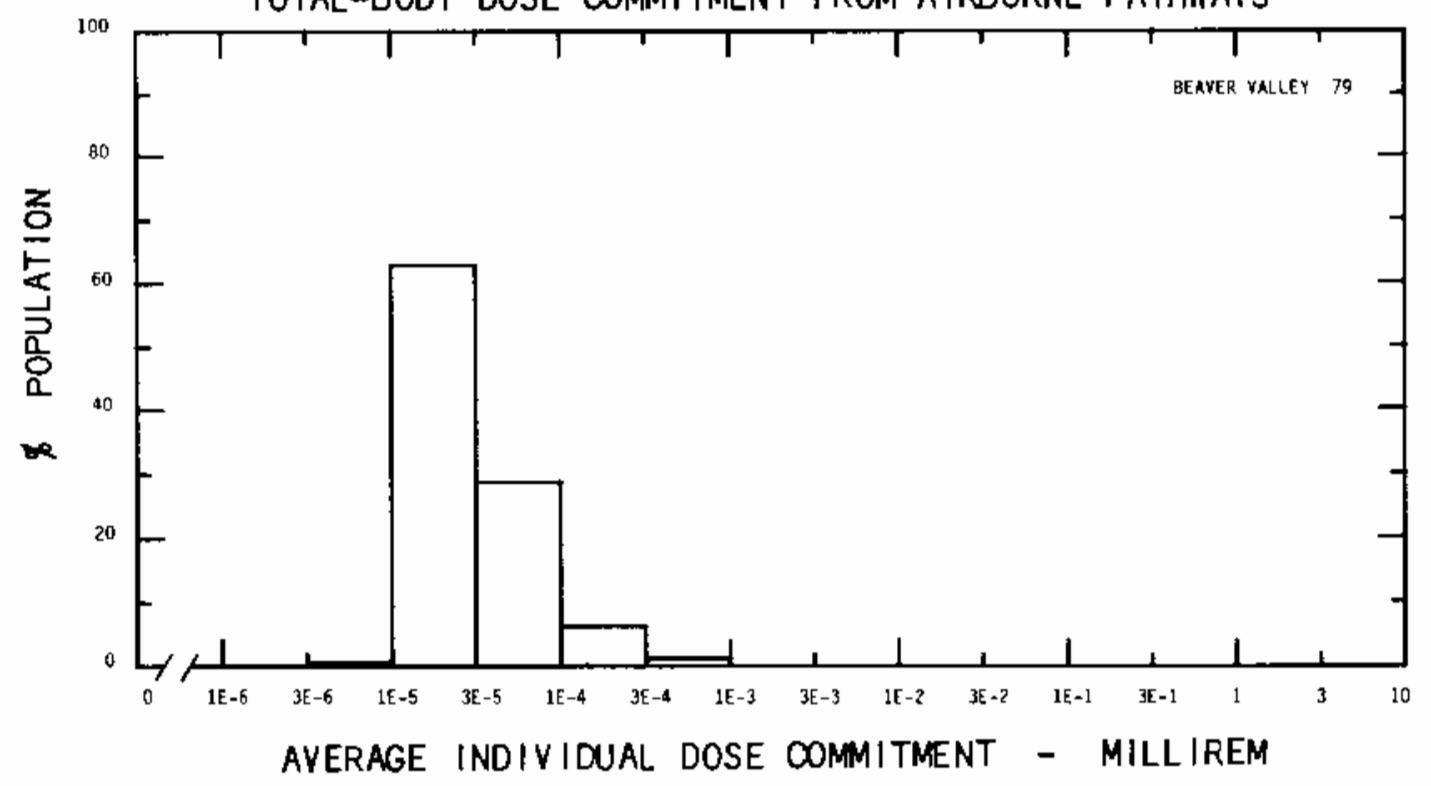




Lecation: $\quad N 45.3589^{\circ}$ W $85.1958^{\circ}$

POPULATION DATA

Total Population within 20-to-80-km Reglon: 1.3E5

Major Metropolitan Centers Within Region:

Center

Pepulation

Lecation

Traverse city

19,000

$70 \mathrm{~km} \quad$ SSW

SITE SPECIFIC DATA - AIRBORNE PATHWAYS

Average Annual State Production

of Crops and AnImal Products

In $80-\mathrm{km}$ Radius Circle

Regional Productivity Factor:

Animal Grazing Factor:

Meteorology Period of Record: 9 FEB 61 - 8 FEB 63
Veg: 6.9E7 kilogram

MIlk: 2.9E8 I iter

Meat: 4.5E7 kilogram

0.5

0.5

SITE SPECIFIC DATA - WATERBORNE PATHWAYS VIO LAKE MICHIGAN
Average Dilution Flow
from Plant: $73 \mathrm{ft}^{3} / \mathrm{s}$
Drinking water:
Exposed Population: $24,0, p^{(a)}$
Dilution Factor: $4.9 E-5$
Fish:
Edible Harvest: - $(\mathrm{cl} \ldots \mathrm{kg} / \mathrm{yr}$ Dilution Factor: 0.01

(a) Population exposed to contaminated drinking water derived from information obtalned from J. Mennigan, Division of Radlation Health, Bureau of Environment and Health, Michigan Department of Public Health.

(b) Drinking water dilution factor estimated by averaging dilution factor derlved from Figure 6B-5, Vol. 1 of WASH-1258 (1973) sultably weighted for population.

(c) Generic consumption rate used (Table A-1). 
POPULATION DOSE-COMMITMENT ESTIMATES AND

AVERAGE INDIVIDUAL DOSE-COMMITMENT HISTOGRAM FOR

\section{BIG ROCK POINT}

Lose Commitments (person-rem) from Liquid Pathways

Iotal Body GL-LLI Ihyroid Bone Live

$\begin{array}{llllll}\text { Infant } & 1.5 \mathrm{E}-06 & 3.2 \mathrm{E}-07 & 9.3 \mathrm{E}-08 & 1.3 \mathrm{E}-05 & 1.5 \mathrm{E}-05 \\ \text { Child } & 3.7 \mathrm{E}-01 & 1.8 \mathrm{E}-02 & 8.2 \mathrm{E}-06 & 2.3 \mathrm{E}-00 & 2.4 \mathrm{E}-00 \\ \text { Teen } & 7.1 \mathrm{E}-01 & 3.4 \mathrm{E}-02 & 6.8 \mathrm{E}-06 & 1.4 \mathrm{E}-00 & 2.0 \mathrm{E}-00 \\ \text { Adult } & 7.8 \mathrm{E}+00 & 2.8 \mathrm{E}-01 & 5.4 \mathrm{E}-05 & 8.0 \mathrm{E}+00 & 1.2 \mathrm{E}+01 \\ \text { TOTAL } & 8.9 \mathrm{E}+00 & 3.3 \mathrm{E}-01 & 6.9 \mathrm{E}-05 & 1.2 \mathrm{E}+01 & 1.6 \mathrm{E}+01\end{array}$

Dose Commitments (person-rem) from Alrborne Pathways TotalBody Gl-LLL Ihyroid Bone Liver Lung

$\begin{array}{lllllll}\text { Infant } & 1.8 \mathrm{E}-03 & 1.8 \mathrm{E}-03 & 1.8 \mathrm{E}-03 & 1.8 \mathrm{E}-03 & 1.8 \mathrm{E}-03 & 1.8 \mathrm{E}-03 \\ \text { Child } & 2.0 \mathrm{E}-02 & 2.0 \mathrm{E}-02 & 2.0 \mathrm{E}-02 & 2.0 \mathrm{E}-02 & 2.0 \mathrm{E}-02 & 2.0 \mathrm{E}-02 \\ \text { Teen } & 1.4 \mathrm{E}-02 & 1.4 \mathrm{E}-02 & 1.5 \mathrm{E}-02 & 1.4 \mathrm{E}-02 & 1.4 \mathrm{E}-02 & 1.5 \mathrm{E}-02 \\ \text { Adult } & 8.8 \mathrm{E}-02 & 8.8 \mathrm{E}-02 & 8.8 \mathrm{E}-02 & 8.8 \mathrm{E}-02 & 8.8 \mathrm{E}-02 & 9.0 \mathrm{E}-02 \\ \text { TOTAL } & 1.2 \mathrm{E}-01 & 1.2 \mathrm{E}-01 & 1.2 \mathrm{E}-01 & 1.2 \mathrm{E}-01 & 1.2 \mathrm{E}-01 & 1.3 \mathrm{E}-01\end{array}$

Production/Consumption factors:

Produce: $1.4 \quad$ Milk: $8.4 \quad$ Meat: 2.1

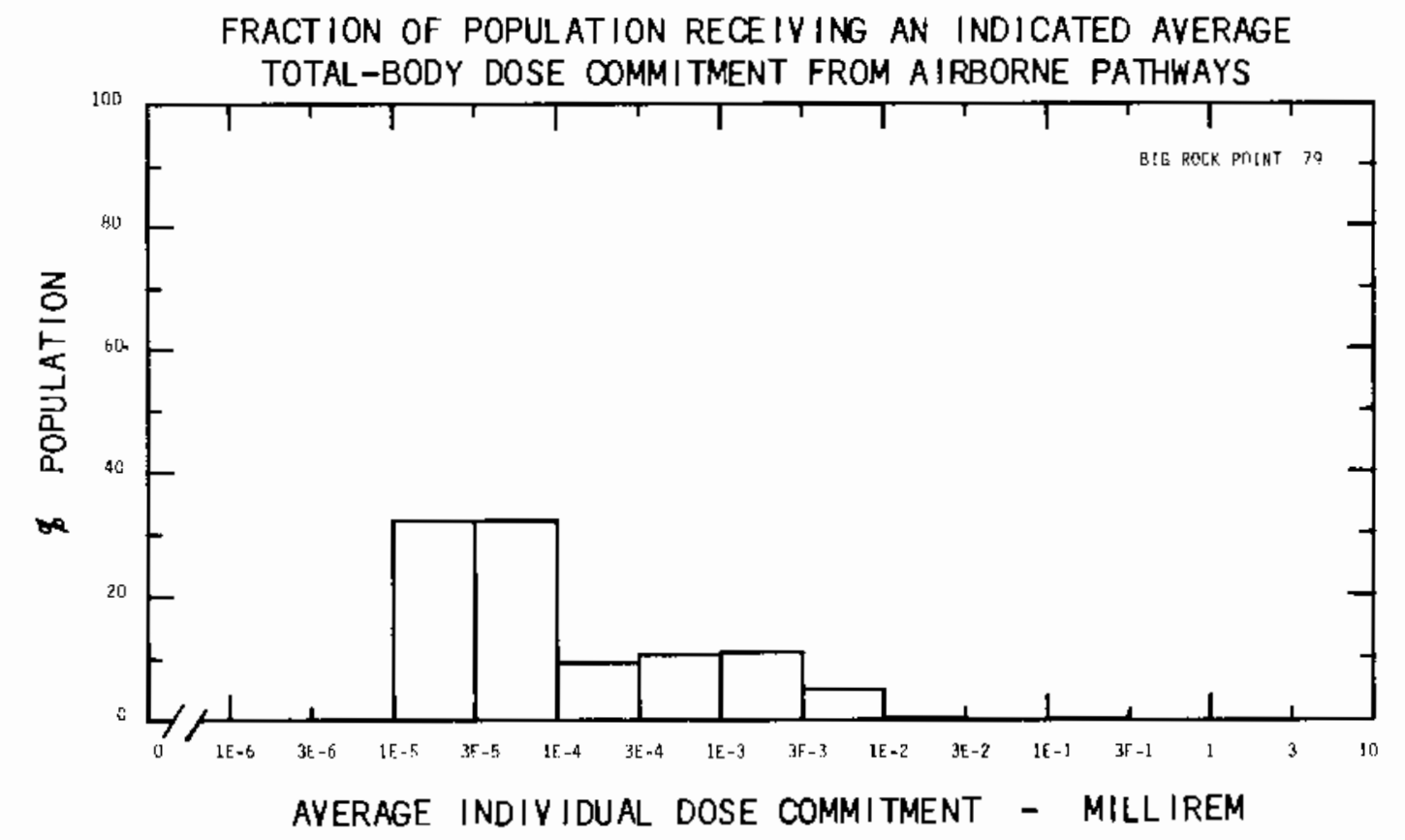


Site: BRONNS FERRY

Lecation: N $34.7036^{\circ}$ W $87.1211^{\circ}$

POPULATION DATA

Total Population Within 20-to-80-km Region: 6.8E5

Major Metropolitan Centers Within Region:

Center

Decatur

Huntsville
Population

42,000

150,000
Lecation

$16 \mathrm{~km} \quad \mathrm{SE}$

$48 \mathrm{~km} \quad \mathrm{E}$

SITE SPECIFIC DATA - AIRBORNE PATHWAYS

Average Annual State Production

of Crops and Animal Products

In 80-km Radius Circle

Reglonal Productivity Factor:

Animal Grazing Factor:

Meteorology Perlod of Record: 1 JAN 74 - 31 DEC 75 Recovery: $94 \%$
Veg: 1.7E7 kllogram

MIlk: 5.7E7 I Iter

Meat: 8.6E7 kllogram

1

0.7

SITE SPECIFIC DATA - WATERBORNE PATHWAYS vIa TENNESSEE RIVER a† WHEELER LAKE

Average River Flow

at Site: $45,000 \mathrm{ft}^{3} / \mathrm{s}$

Drinking water:

Exposed Population: 23,000

Dilution Factor: 1

Fish:

Edible Harvest: $1.6 E 6 \mathrm{~kg} / \mathrm{yr}$

Dilution Factor: 1 


\section{POPULATION DOSE-COMMITMENT ESTIMATES AND AVERAGE INDIVIDUAL DOSE-COMMITMENT HISTOGRAM FOR}

BRONS FERRY 1, 2 AMD 3

Dose Comm Itments (person-rem) from Liquid Pathways Iotal Body Ihyrold Bone Llyer

$\begin{array}{llllll}\text { Infant } & 8.6 \mathrm{E}-05 & 4.3 \mathrm{E}-05 & 6.1 \mathrm{E}-03 & 3.3 \mathrm{E}-04 & 3.9 \mathrm{E}-04 \\ \text { Child } & 1.5 \mathrm{E}-01 & 1.8 \mathrm{E}-01 & 8.0 \mathrm{E}-02 & 4.1 \mathrm{E}-01 & 6.7 \mathrm{E}-01 \\ \text { Teen } & 2.8 \mathrm{E}-01 & 3.7 \mathrm{E}-01 & 4.0 \mathrm{E}-02 & 2.6 \mathrm{E}-01 & 5.9 \mathrm{E}-01 \\ \text { Adult } & 2.9 \mathrm{E}+00 & 3.2 \mathrm{E}+00 & 2.7 \mathrm{E}-01 & 1.5 \mathrm{E}+00 & 3.6 \mathrm{E}+00 \\ \text { TOTAL } & 3.3 \mathrm{E}+00 & 3.8 \mathrm{E}+00 & 4.0 \mathrm{E}-01 & 2.2 \mathrm{E}+00 & 4.9 \mathrm{E}+00\end{array}$

Dose Commitments (person-rem) from Airborne Pathways IotalBody Gl-LL Ihyrold Bone Llyer Lung

$\begin{array}{lllllll}\text { Infant } & 8.7 \mathrm{E}-02 & 1.9 \mathrm{E}-01 & 8.1 \mathrm{E}-02 & 3.4 \mathrm{E}-01 & 7.4 \mathrm{E}-02 & 3.4 \mathrm{E}+00 \\ \text { ChIld } & 1.2 \mathrm{E}+00 & 5.6 \mathrm{E}+00 & 8.6 \mathrm{E}-01 & 7.3 \mathrm{E}+00 & 8.2 \mathrm{E}-01 & 4.1 \mathrm{E}+01 \\ \text { Teen } & 7.5 \mathrm{E}-01 & 6.8 \mathrm{E}+00 & 6.2 \mathrm{E}-01 & 3.0 \mathrm{E}+00 & 6.0 \mathrm{E}-01 & 3.5 \mathrm{E}+01 \\ \text { Adult } & 4.3 \mathrm{E}+00 & 3.9 \mathrm{E}+01 & 3.7 \mathrm{E}+00 & 1.4 \mathrm{E}+01 & 3.6 \mathrm{E}+00 & 1.3 \mathrm{E}+02 \\ \text { TOTAL } & 6.3 \mathrm{E}+00 & 5.1 \mathrm{E}+01 & 5.2 \mathrm{E}+00 & 2.4 \mathrm{E}+01 & 5.1 \mathrm{E}+00 & 2.1 \mathrm{E}+02\end{array}$

Production/Consumption factors:

Produce: $<1 \quad$ Milk: $<1 \quad$ Meat: 1.6

FRACTION OF POPULATION RECEIVING AN INDICATED AVERAGE TOTAL-BODY DOSE OOMMITMENT FROM AIRBORNE PATHWAYS

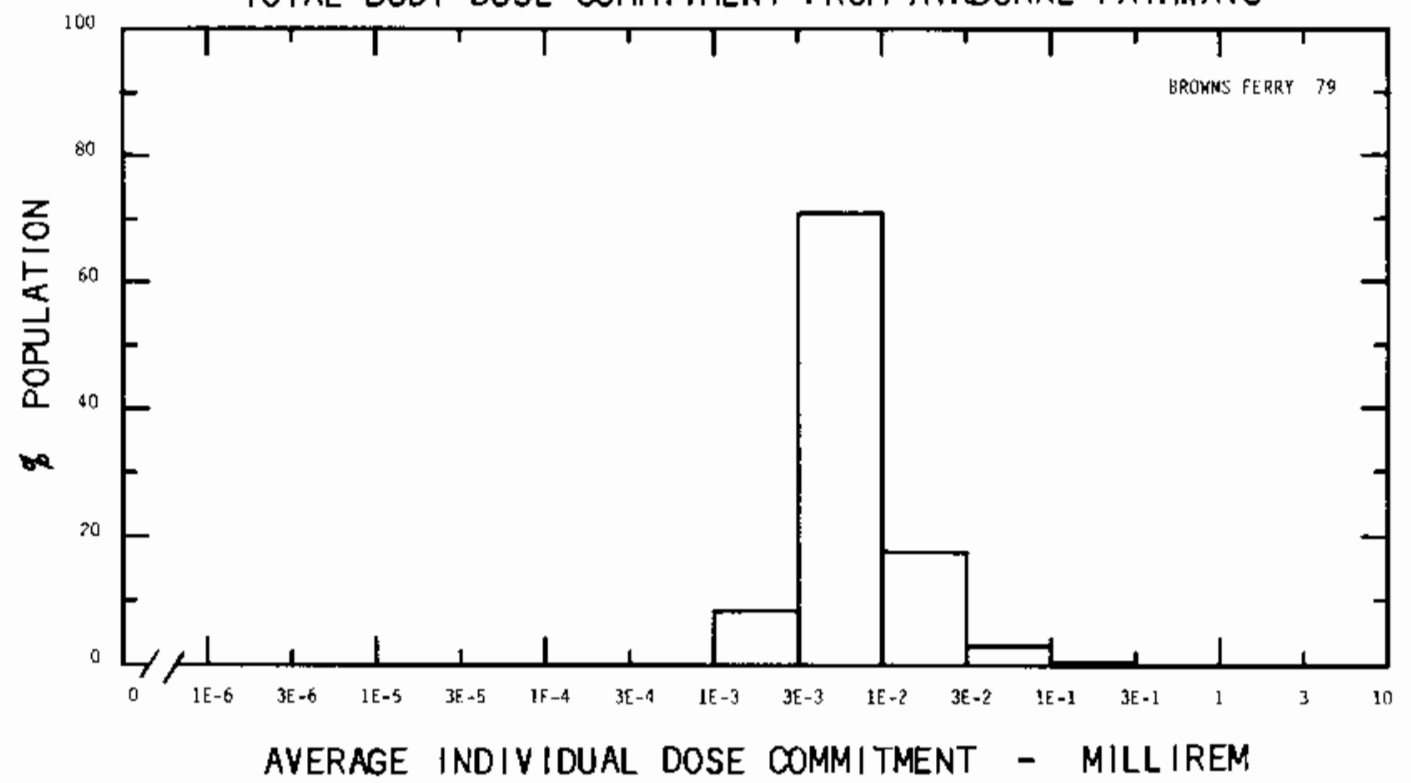


Site: BRUNSUICK

Location: $\quad \mathrm{N} 33.9586^{\circ}$
BRUNSWICK COUNTY, NORTH CAROL IM

W $78.0208^{\circ}$

POPULATION DATA

Total Population Within 20-to-80-km Region: 1.9E5

Major Metropolitan Centers with In Reglon:

Center

Wi InIngton
Population

51,000
Location

$32 \mathrm{~km} \quad \mathrm{~N}$

SITE SPECIFIC DATA - AIRBORNE PATHWAYS

Average Annual State Production

of Crops and Animal Products

In 80-km Radius Circle

Reglonal Productivity Factor:

Animal Grazing Factor:

Meteorology Period of Record: 1
Veg: $2.6 \mathrm{E7}$ kllogram

Milk: 1,0E8 liter

Meat: $5.8 \mathrm{E} 7 \mathrm{kilogram}$

0.3

0.7

SITE SPECIFIC DATA - WATERBORNE PATHWAYS vIa ATLANTIC OCEAN
Average Dilution Flow
from Plant: $240 \mathrm{ft}^{3} / \mathrm{s}$
FIsh:
Edible Harvest: $2.15^{(a)} \mathrm{kg} / \mathrm{yr}$
Dilution Factor: $\mathbf{0 . 0 0 1}$
Invertebrates:
Edible Harvest: $1.1 \mathrm{Es}^{(\mathrm{a})} \mathrm{kg} / \mathrm{yr}$
Dilution Factor: 0.002

(a) Fish and invertebrate harvests together total harvest given in FES (1973). 


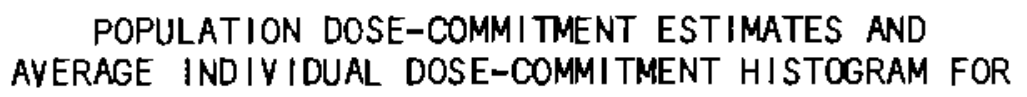

BRUNSWICK 1 AND 2

Infant

Child

Teen

Adult

TOTAL

Iotal Body

Gl-LلL

Ihyredd

Bone

Liver

$\begin{array}{lllll}0.0 \mathrm{E}+00 & 0.0 \mathrm{E}+00 & 0.0 \mathrm{E}+00 & 0.0 \mathrm{E}+00 & 0.0 \mathrm{E}+00 \\ 2.8 \mathrm{E}-03 & 2.9 \mathrm{E}-03 & 2.6 \mathrm{E}-03 & 2.5 \mathrm{E}-03 & 5.0 \mathrm{E}-03 \\ 2.1 \mathrm{E}-03 & 5.9 \mathrm{E}-03 & 1.8 \mathrm{E}-03 & 1.6 \mathrm{E}-03 & 4.4 \mathrm{E}-03 \\ 1.5 \mathrm{E}-02 & 5.2 \mathrm{E}-02 & 1.2 \mathrm{E}-02 & 9.9 \mathrm{E}-03 & 2.7 \mathrm{E}-02 \\ 2.0 \mathrm{E}-02 & 6.1 \mathrm{E}-02 & 1.7 \mathrm{E}-02 & 1.4 \mathrm{E}+02 & 3.6 \mathrm{E}-02\end{array}$

Dose Commitments (person-rem) from Alrborne Pathways

Total Body Gl-LLl Thyrold Bone Llyer Lung

$\begin{array}{lllllll}\text { Infant } & 3.2 \mathrm{E}-02 & 3.2 \mathrm{E}-02 & 6.5 \mathrm{E}-02 & 3.2 \mathrm{E}-02 & 3.2 \mathrm{E}-02 & 3.3 \mathrm{E}-02 \\ \text { ChIId } & 3.5 \mathrm{E}-01 & 3.5 \mathrm{E}-01 & 5.6 \mathrm{E}-01 & 3.5 \mathrm{E}-01 & 3.5 \mathrm{E}-01 & 3.7 \mathrm{E}-01 \\ \text { Teen } & 2.6 \mathrm{E}-01 & 2.6 \mathrm{E}-01 & 3.4 \mathrm{E}-01 & 2.6 \mathrm{E}-01 & 2.6 \mathrm{E}-01 & 2.8 \mathrm{E}-01 \\ \text { Adult } & 1.6 \mathrm{E}+00 & 1.6 \mathrm{E}+00 & 1.9 \mathrm{E}+00 & 1.6 \mathrm{E}+00 & 1.6 \mathrm{E}+00 & 1.6 \mathrm{E}+00 \\ \text { TOTAL } & 2.2 \mathrm{E}+00 & 2.2 \mathrm{E}+00 & 2.8 \mathrm{E}+00 & 2.2 \mathrm{E}+00 & 2.2 \mathrm{E}+00 & 2.3 \mathrm{E}+00\end{array}$

Production/Consumption factors:

Produce: <1 Milk: $1.2 \quad$ Meat: 1.1

FRACTION OF POPULATION RECEIVING AN INDICATED AVERAGE TOTAL-BODY DOSE COMMITMENT FROM AIRBORNE PATHWAYS

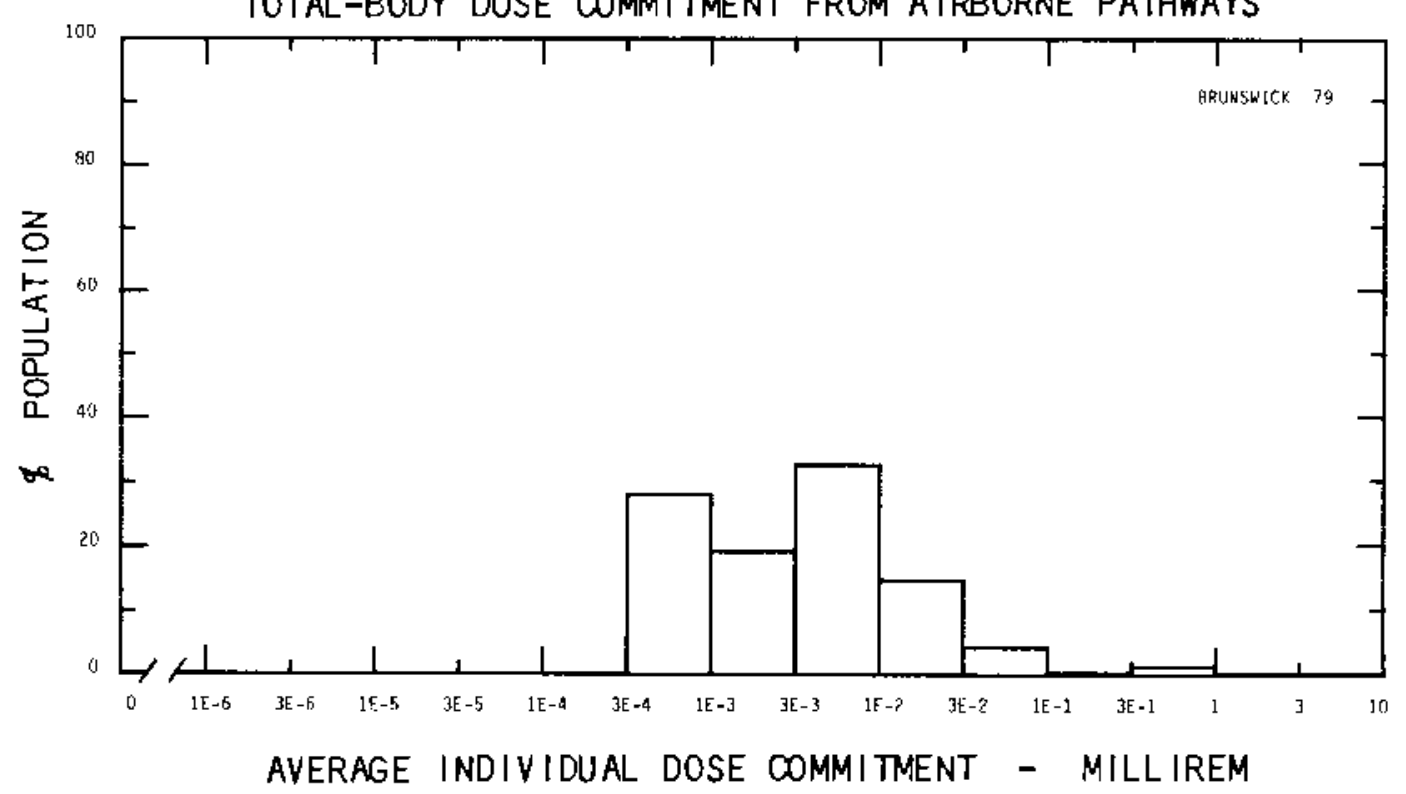


SIte: CNLERT CLIFFS

LUSBY, MARYLAND

Location:

N $38.4272^{\circ}$

W $76.4261^{\circ}$

POPULATION DATA

Total Population Within 20-to-80-km Region: 2.4E6

Major Metropolitan Centers Within Reglon:

Center

Washington, DC SMSA (3/4)

Canbridge

Annapol is
Population

$2,300,000$

12,000

31,000
Location

$75 \mathrm{~km} \quad \mathrm{MH}$

$32 \mathrm{~km}$ ENE

$61 \mathrm{~km} \mathrm{~N}$

SITE SPECIFIC DATA - AIRBORNE PATHWAYS

Average Annual State Production

Of Crops and Animal Products

In 80-km Radius Circle

Regional Productivity Factor:

AnImal Grazing Factor:

Meteorology Perlod of Record: 1 JN 75 - 31 DEC 75 Recovery: 96\$
Veg: 4.5E7 kllogram

Milk: 5.0E8 liter

Meat: 6.2E7 kilogram

0.6

0.6

SITE SPECIFIC DATA - WATERBORNE PATHWAYS VIa CHESAPEAKE BAY

$\begin{array}{ll} & \begin{array}{l}\text { Average Dilution Flow } \\ \text { from Plant: } 2,500 \mathrm{ft} / \mathrm{s}\end{array} \\ & \text { Edible Harvest: } 1.0 \mathrm{E} 7 \mathrm{~kg}\langle\mathrm{yr} \\ & \text { Dilution Factor: } 0.062 \\ & \text { Edible Harvest: } 7.4 \mathrm{Eg} \mathrm{kg} / \mathrm{yr} \\ & \text { Dilution Factor: } 0.062(\mathrm{a})\end{array}$

a) Dilutions given in FES (1973). 


\section{POPULATION DOSE-COMMITMENT ESTIMATES AND AVERAGE INDIVIDUAL. DOSE-COMMITMENT HISTOGRAM FOR \\ CALVERT CLIFFS 1 NO 2}

Dose Commitments (person-rem) from Llquid Pathways

Iotal Body G.JLL Thyrold Bone Liver

$\begin{array}{llllll}\text { Infant } & 0.0 \mathrm{E}+00 & 0.0 \mathrm{E}+00 & 0.0 \mathrm{E}+00 & 0.0 \mathrm{E}+00 & 0.0 \mathrm{E}+00 \\ \text { Child } & 2.2 \mathrm{E}-01 & 7.2 \mathrm{E}-01 & 7.0 \mathrm{E}-01 & 2.7 \mathrm{E}-01 & 3.9 \mathrm{E}-01 \\ \text { Teen } & 2.1 \mathrm{E}-01 & 1.5 \mathrm{E}+00 & 4.9 \mathrm{E}-01 & 1.6 \mathrm{E}-01 & 3.4 \mathrm{E}-01 \\ \text { Adult } & 1.7 \mathrm{E}+00 & 1.3 \mathrm{E}+01 & 3.2 \mathrm{E}+00 & 9.5 \mathrm{E}-01 & 2.0 \mathrm{E}+00 \\ \text { TOTAL } & 2.1 \mathrm{E}+00 & 1.6 \mathrm{E}+01 & 4.4 \mathrm{E}+00 & 1.4 \mathrm{E}+00 & 2.8 \mathrm{E}+00\end{array}$

Dose Conmitments (person-rem) from Airborne Pathways Iotal Body Gl-LLl Thyrold Bone Liver Lung

$\begin{array}{lllllll}\text { Infant } & 1.3 \mathrm{E}-02 & 4.7 \mathrm{E}-03 & 3.8 \mathrm{E}-01 & 4.9 \mathrm{E}-02 & 5.4 \mathrm{E}-03 & 8.4 \mathrm{E}-03 \\ \text { ChIId } & 2.3 \mathrm{E}-01 & 5.8 \mathrm{E}-02 & 2.0 \mathrm{E}+00 & 1.0 \mathrm{E}+00 & 5.3 \mathrm{E}-02 & 1.1 \mathrm{E}-01 \\ \text { Teen } & 1.2 \mathrm{E}-01 & 4.5 \mathrm{E}-02 & 8.0 \mathrm{E}-01 & 5.7 \mathrm{E}-01 & 3.7 \mathrm{E}-02 & 8.6 \mathrm{E}-02 \\ \text { AduIt } & 5.5 \mathrm{E}-01 & 2.5 \mathrm{E}-01 & 2.6 \mathrm{E}+00 & 2.7 \mathrm{E}+00 & 2.1 \mathrm{E}-01 & 3.9 \mathrm{E}-01 \\ \text { TOTAL } & 9.2 \mathrm{E}-01 & 3.6 \mathrm{E}-01 & 5.8 \mathrm{E}+00 & 4.4 \mathrm{E}+00 & 3.1 \mathrm{E}-01 & 5.9 \mathrm{E}-01\end{array}$

Production/Consumption factors:

Produce: $<1$ Milk: $<1 \quad$ Meat: $<1$

FRACTION OF POPULATION RECEIVING AN INDICATED AVERAGE TOTAL-BODY DOSE COMMITMENT FROM AIRBORNE PATHWAYS

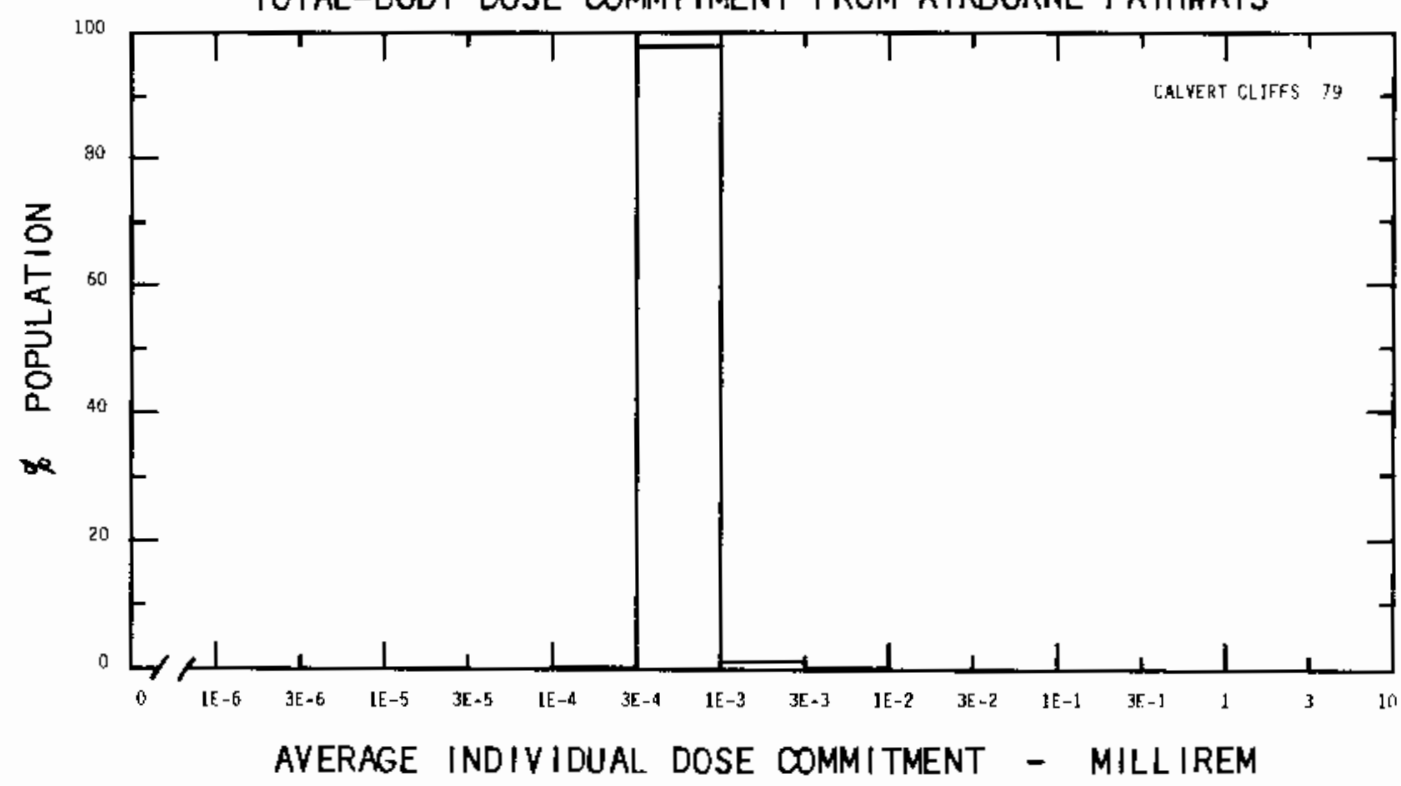


Site: cook

Location:
N $41.9742^{\circ}$
BENTON HARBOR, MICHIGNN

W $86.5667^{\circ}$

POPULATION DATA

Total Population within 20-to-80-km Reglon: 1.1 E6

Major Metropolitan Centers within Region:

Center

Gary SMSA

South Bend SMSA

Benton Harbor
Population

660,000

290,000

17,000
Location

$80 \mathrm{~km} \mathrm{SH}$

$42 \mathrm{~km} \quad \mathrm{SE}$

$18 \mathrm{~km} \quad \mathrm{NE}$

SITE SPECIFIC DATA - AIRBORNE PATHWAYS

Average Annual State Production

of Crops and Animal Products

In 80-km Radlus Circle

Reglonal Productivity Factor:

AnImal Grazing Factor:

Meteorology Perlod of Record: 1 MAY 75 - 31 APR 76 Recovery: 95\%
Veg: $1.1 \mathrm{E8} \mathrm{kilogram}$

Milk: 2.3EB liter

Meat: 1.9E8 kilogram

0.6

0.5

SITE SPECIFIC DATA - WATERBORNE PATHWAYS VIa LAKE MIOHIGAN

$\begin{array}{ll} & \begin{array}{l}\text { Average DIlution Elow } \\ \text { from Plant: } 92 \mathrm{ft} / \mathrm{s}\end{array} \\ \text { Drinking Water: } & \begin{array}{l}\text { Exposed Population: } 279,000 \\ \text { Dilution Factor: } 0.025\end{array} \\ & \text { Edible Harvest: } 1.5 \mathrm{~kg} \mathrm{~kg} / \mathrm{yr} \\ & \text { Dilution Factor: } 0.01\end{array}$

(a) Drinking water dilution factor estimated by averagling dilution factors derived from FES (1973) suitably welghted for population. 
POPULATION DOSE-COMMITMENT ESTIMATES AND

AVERAGE INDIVIDUAL DOSE-COMMITMENT HISTOGRAM FOR

COOK 1 ND 2

Dose Cormitments (person-rem) from LIquid Pathways

Iotal Body Ihyrold Bone Luer

$\begin{array}{llllll}\text { Infant } & 8.4 \mathrm{E}-02 & 8.1 \mathrm{E}-02 & 1.1 \mathrm{E}-01 & 3.4 \mathrm{E}-02 & 1.3 \mathrm{E}-01 \\ \text { Child } & 1.5 \mathrm{E}+00 & 1.2 \mathrm{E}+00 & 1.1 \mathrm{E}+00 & 2.6 \mathrm{E}+00 & 4.1 \mathrm{E}+00 \\ \text { Teen } & 1.4 \mathrm{E}+00 & 8.8 \mathrm{E}-01 & 3.9 \mathrm{E}-01 & 1.5 \mathrm{E}+00 & 2.9 \mathrm{E}+00 \\ \text { Adult } & 1.4 \mathrm{E}+01 & 7.7 \mathrm{E}+00 & 3.3 \mathrm{E}+00 & 8.5 \mathrm{E}+00 & 1.8 \mathrm{E}+01 \\ \text { TOTAL } & 1.7 \mathrm{E}+01 & 9.8 \mathrm{E}+00 & 4.8 \mathrm{E}+00 & 1.3 \mathrm{E}+01 & 2.5 \mathrm{E}+01\end{array}$

Dose Comm I tments (person-rem) from Airborne Pathways

Total Body Gl-LLل Ihyrold Bone Luer Lung

$\begin{array}{lllllll}\text { Infant } & 6.5 \mathrm{E}-04 & 6.3 \mathrm{E}-04 & 7.6 \mathrm{E}-03 & 7.1 \mathrm{E}-04 & 7.4 \mathrm{E}-04 & 8.5 \mathrm{E}-04 \\ \text { ChIld } & 7.8 \mathrm{E}-03 & 7.5 \mathrm{E}-03 & 5.4 \mathrm{E}-02 & 9.2 \mathrm{E}-03 & 8.1 \mathrm{E}-03 & 1.0 \mathrm{E}-02 \\ \text { Teen } & 5.5 \mathrm{E}-03 & 5.6 \mathrm{E}-03 & 2.3 \mathrm{E}-02 & 5.8 \mathrm{E}-03 & 5.6 \mathrm{E}-03 & 9.1 \mathrm{E}-03 \\ \text { Adult } & 3.3 \mathrm{E}-02 & 3.4 \mathrm{E}-02 & 9.1 \mathrm{E}-02 & 3.3 \mathrm{E}-02 & 3.2 \mathrm{E}-02 & 4.4 \mathrm{E}-02 \\ \text { TOTAL } & 4.6 \mathrm{E}-02 & 4.8 \mathrm{E}-02 & 1.8 \mathrm{E}-01 & 4.9 \mathrm{E}-02 & 4.7 \mathrm{E}-02 & 6.4 \mathrm{E}-02\end{array}$

Production/Consumption factors:

Produce: $<1 \quad$ Milk: $<1 \quad$ Meat: 1.3

FRACTION OF POPULATION RECEIVING AN INDICATED AVERAGE TOTAL-BODY DOSE COMMITMENT FROM AIRBORNE PATHWAYS

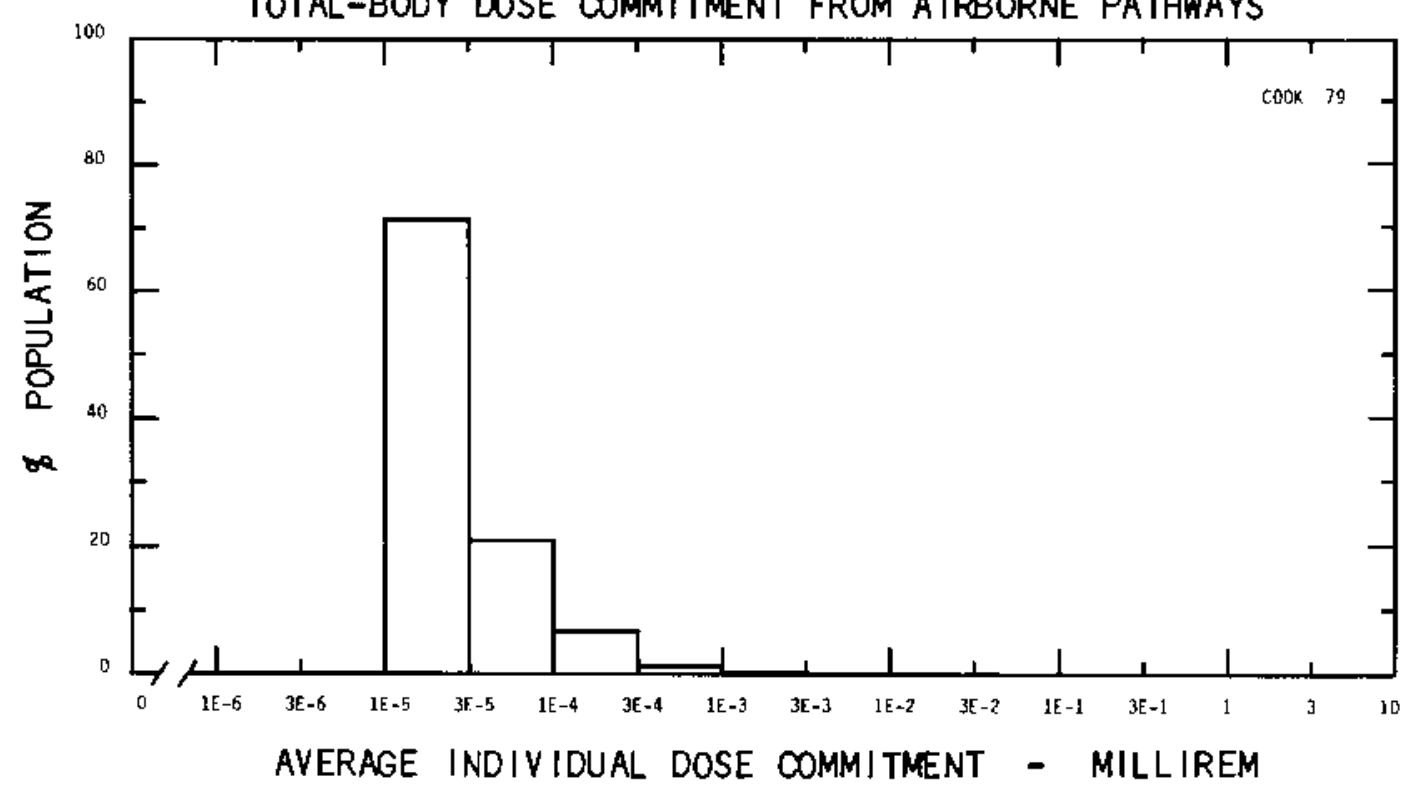


SLIE: COOPER STATION

Lecation: $\quad \mathrm{N} \quad 40.3614^{\circ}$
NEWHA COUNTY, NEBRASKA

W $95.6378^{\circ}$

POPULATION DATA

Total Population Within 20-to-80-km Reglon: 1.8E5

Major Metropolitan Centers Within Region:

Center

Shenandoah
Population

6,300
Location

$58 \mathrm{~km}$ NE

SITE SPECIFIC DATA - AIRBORNE PATHWAYS

Average Annual State Production

Of Crops and Anjmal Products

In 80-km Radius Circle

Reglonal Productivity Factor:

Animal GrazIng Factor:

Meteorology Period of Record:
Veg: 9.7E7 kilogram

Milk: 7.2E7 liter

Meat: 2.0E8 kilogram

1

0.6

1 MAR 70 - 31 DEC 75 Recovery: 89\%

Drinking Water:

Fish :
Average River Flow

at Site: $31,000 \mathrm{ft}^{3} / \mathrm{s}$

Exposed Population: None

Edible Harvest: $5.0 \mathrm{G}^{(\mathrm{a})} \mathrm{kg} / \mathrm{yr}$

(a) Assumes $1 / 2$ fish harvest caught below plant. 


\section{POPULATION DOSE-COMMITMENT ESTIMATES AND AVERAGE INDIVIDUAL DOSE-COMMITMENT HISTOGRAM FOR COOPER STATION}

$\begin{array}{llllll}\text { Infant } & 0.0 \mathrm{E}+00 & 0.0 \mathrm{E}+00 & 0.0 \mathrm{E}+00 & 0.0 \mathrm{E}+00 & 0.0 \mathrm{E}+00 \\ \text { Child } & 3.4 \mathrm{E}-04 & 4.0 \mathrm{E}-05 & 8.5 \mathrm{E}-06 & 1.5 \mathrm{E}-03 & 1.7 \mathrm{E}-03 \\ \text { Teen } & 6.0 \mathrm{E}-04 & 8.5 \mathrm{E}-05 & 6.2 \mathrm{E}-06 & 8.9 \mathrm{E}-04 & 1.5 \mathrm{E}-03 \\ \text { Adult } & 6.4 \mathrm{E}-03 & 7.4 \mathrm{E}-04 & 4.1 \mathrm{E}-05 & 5.2 \mathrm{E}-03 & 8.8 \mathrm{E}-03 \\ \text { TOTAL } & 7.3 \mathrm{E}-03 & 8.7 \mathrm{E}-04 & 5.5 \mathrm{E}-05 & 7.5 \mathrm{E}-03 & 1.2 \mathrm{E}-02\end{array}$

Dose Commltments (person-rem) from Alrborne Pathways Iotal Body Gl-LLl Thyroid Bone Llyer Lung

$\begin{array}{lllllll}\text { Infant } & 4.8 \mathrm{E}-03 & 4.8 \mathrm{E}-03 & 1.2 \mathrm{E}-02 & 4.8 \mathrm{E}-03 & 4.8 \mathrm{E}-03 & 5.0 \mathrm{E}-03 \\ \text { ChIld } & 5.4 \mathrm{E}-02 & 5.4 \mathrm{E}-02 & 1.3 \mathrm{E}-01 & 5.4 \mathrm{E}-02 & 5.4 \mathrm{E}-02 & 5.6 \mathrm{E}-02 \\ \text { Teen } & 3.9 \mathrm{E}-02 & 3.9 \mathrm{E}-02 & 6.7 \mathrm{E}-02 & 3.9 \mathrm{E}-02 & 3.9 \mathrm{E}-02 & 4.2 \mathrm{E}-02 \\ \text { Adult } & 2.4 \mathrm{E}-01 & 2.4 \mathrm{E}-01 & 3.4 \mathrm{E}-01 & 2.4 \mathrm{E}-01 & 2.4 \mathrm{E}-01 & 2.5 \mathrm{E}-01 \\ \text { TOTAL } & 3.4 \mathrm{E}-01 & 3.4 \mathrm{E}-01 & 5.4 \mathrm{E}-01 & 3.4 \mathrm{E}-01 & 3.4 \mathrm{E}-01 & 3.5 \mathrm{E}-01\end{array}$

Production/Consumption factors:

Produce: $2.8 \quad$ Milk: 3.0 Meat: 14.0

FRACTION OF POPULATION RECEIVING AN INDICATED AVERAGE TOTAL-BODY DOSE COMMITMENT FROM AIRBORNE PATHWAYS

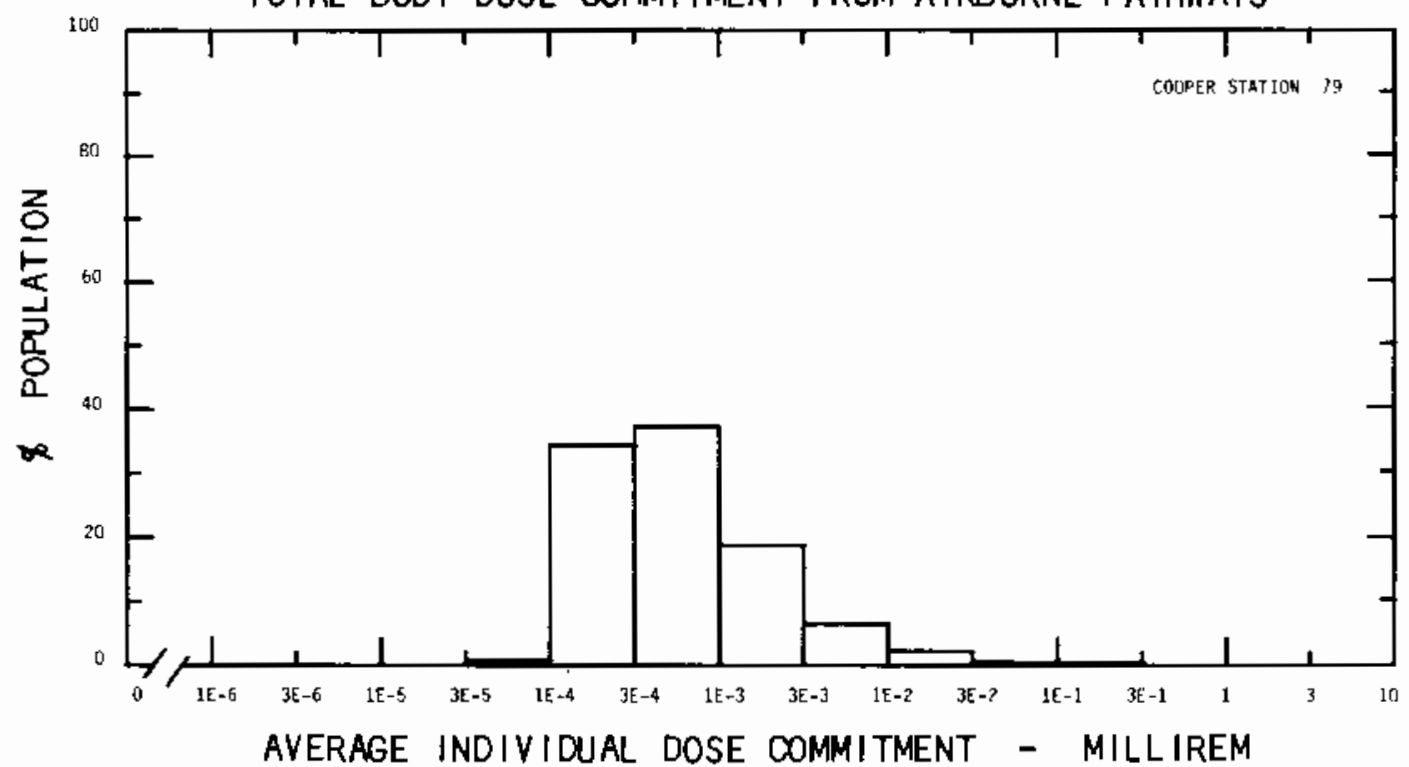


SJte: CRYSTNL RIVER

Location: N $28.9567^{\circ}$
CRYSTAL RIVER, FLORIDA

W $82.6980^{\circ}$

POPULATION DATA

Total Population within 20-to-80-km RegIon: 2.3E5

Major Metropolitan Centers within Region:

Center

Population

Lecation

Ocala

29,000

$63 \mathrm{kin}$ ENE

SITE SPECIFIC DATA - AIRBORNE PATHWAYS

Average Annual State Production

of Crops and Animal Products

In 80-km Radjus Circle

Regional Productivity Factor:

Animal Grazing Factor:

Meteorology Period of Record: 1 JAN 75 - 31 DEC 75 Recovery: 93\%
Veg: $2.8 E 7$ kllogram

MIIK: 1.1E8 I iter

Meat: $7.2 \mathrm{E}$ kilogram

0.5

1

SITE SPECIFIC DATA - WATERBORNE PATHWAYS via GULF Of MEXICO

SITE SPECIFIC DATA - WATERBORNE PATHWAYS VIa GULF OF MEXICO
Average Dilution Fłow
from Plant: $100 \mathrm{ft}^{3} / \mathrm{s}$
Fish:
Edible Harvest: $3.2 E 5 \mathrm{~kg} / \mathrm{yr}$
Dilution Factor: 0.1
Invertebrates:
Edible Harvest: $1.8 E 5 \mathrm{~kg} / \mathrm{yr}$
Dilution Factor: 0.1 


\section{POPULATION DOSE-COMMITMENT ESTIMATES AND AVERAGE INDIVIDUAL DOSE-COMMITMENT HISTOGRAM FOR CRYSTN RIYER}

$\begin{array}{lclll}\text { Dose Comm Itments (person-rem) } & \text { from L lquld Pathways } \\ \text { Total_Body } & \text { GJ-LLL } & \text { Thyrold } & \text { Bone } & \text { Llver } \\ 0.0 \mathrm{E}+00 & 0.0 \mathrm{E}+00 & 0.0 \mathrm{E}+00 & 0.0 \mathrm{E}+00 & 0.0 \mathrm{E}+00 \\ 4.3 \mathrm{E}-02 & 8.7 \mathrm{E}-02 & 1.5 \mathrm{E}-01 & 9.7 \mathrm{E}-02 & 6.9 \mathrm{E}-02 \\ 4.0 \mathrm{E}-02 & 1.8 \mathrm{E}-01 & 1.1 \mathrm{E}-01 & 6.4 \mathrm{E}-02 & 5.9 \mathrm{E}-02 \\ 3.2 \mathrm{E}-01 & 1.5 \mathrm{E}+00 & 7.0 \mathrm{E}-01 & 4.2 \mathrm{E}-01 & 3.6 \mathrm{E}-01 \\ 4.0 \mathrm{E}-01 & 1.8 \mathrm{E}+00 & 9.5 \mathrm{E}-01 & 5.8 \mathrm{E}-01 & 4.8 \mathrm{E}-01\end{array}$

Infant

Child

Teen

Adult

TOTAL

infant

ChIld

Teen

Adult

TOTAL

Dose Commltments (person-rem) from Airborne Pathways

\section{IotalBody GL-LLل Ihyrodd Bone Llyer Lung}

Production/Consumption factors:
Produce: $<1$
Mllk: 1.8
Meat: 1.9

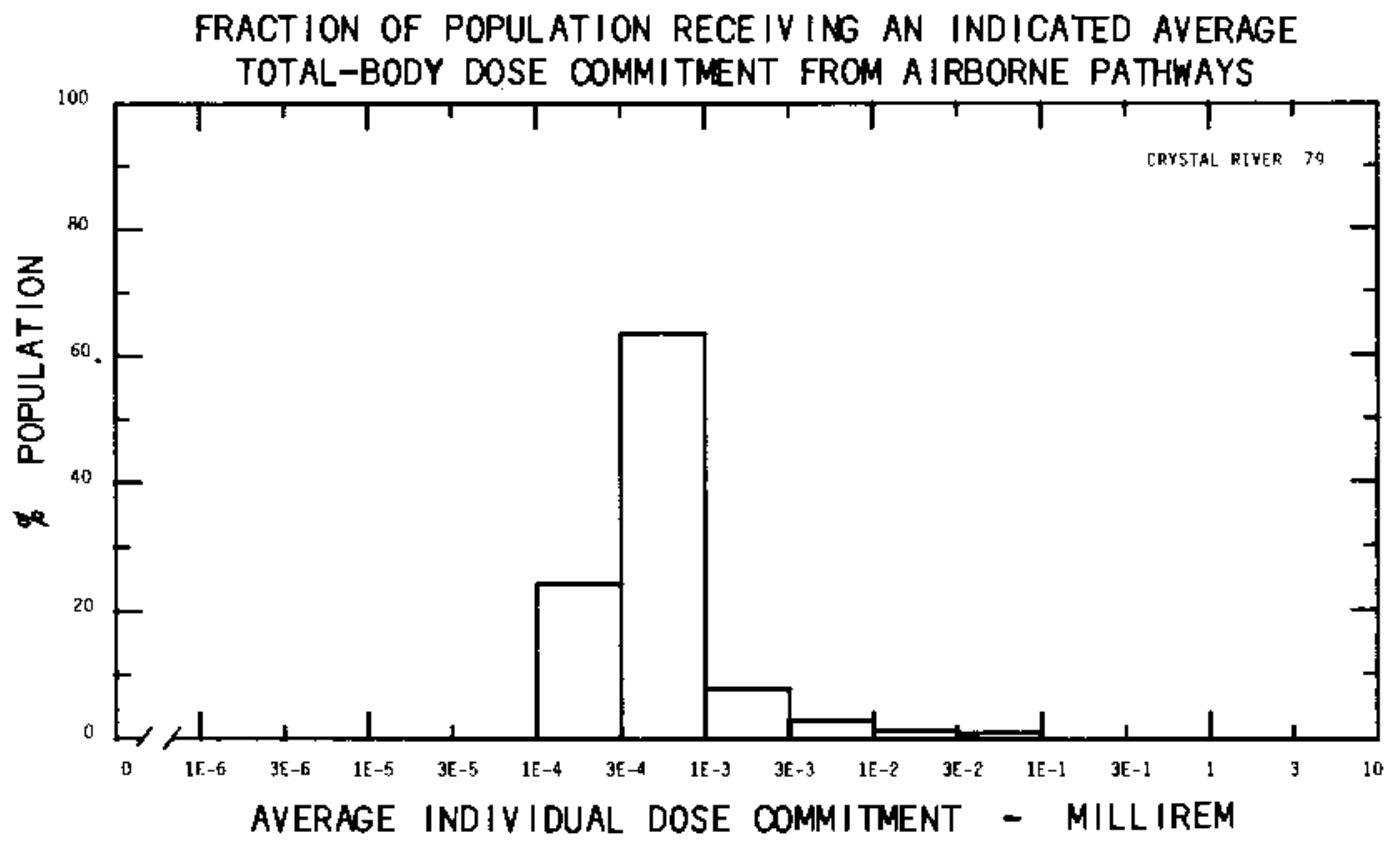


SJte: DAYIS-BESSE

PORT CLINTON, OHIO

Lecation: N $41.5947^{\circ} \quad$ $83.0864^{\circ}$

POPULATION DATA

Total Population Within 20-to-80-km Region: 1.8E6

Major Metropolitan Centers Within Region:

\begin{tabular}{lrrr} 
Center & Population & \multicolumn{2}{c}{ Location } \\
Toledo & 390,000 & $37 \mathrm{~km}$ & WM \\
Sandusky & 33,000 & $35 \mathrm{~km}$ & ESE \\
Findlay & 36,000 & $77 \mathrm{~km}$ & $\mathbf{S W}$ \\
Dearborn & 100,000 & $80 \mathrm{~km}$ & $\mathbf{N}$
\end{tabular}

SITE SPECIFIC DATA - AIRBORNE PATHWAYS

Average Annual State Production

of Crops and Animal Products

In 80-km Radius Clrcle

Regional Productivity Factor:

Animal Grazing Factor:

Meteorology Perlod of Record:
Veg: $6.9 E 7$ kllogram

Mllk: 3.7E8 liter

Meat: 1.2E8 kllogram

0.5

0.5

$80 \mathrm{~km} N$

SITE SPECIFIC DATA - WATERBORNE PATHWAYS VIa LAKE ERIE

Average DIIution Flow

from Plant: $210 \mathrm{ft}^{3} / \mathrm{s}$

Drinking Water:

Exposed Population: 450,000

Dilution Factor: $2.0 \mathrm{E}-4{ }^{(a)}$

Fish :

Edible Harvest: (b) $\mathrm{kg} / \mathrm{yr}$

Dllution Factor: $\mathbf{0 . 0 1}$

(a) Drinking water dilution factor ostimated by averaging dilution factor derlved from FES (1973), sultably welghted for population.

(b) No fIsh catch data given in FES (1973); thus, generic consumption rates used (Table $A-1)$. 
POPULATION DOSE-COMMITMENT ESTIMATES AND

AVERAGE INDIVIDUAL DOSE-COMMITMENT HISTOGRAM FOR

\section{DAVIS-BESSE}

Dose Commitments (person-rem) from Liquid Pathways

Iotal Body $\mathrm{Gl-LL}$ Thyrold Bone Liver

\begin{tabular}{|c|c|c|c|c|c|}
\hline $\begin{array}{l}\text { Infant } \\
\text { Child } \\
\text { Teen } \\
\text { Adult }\end{array}$ & $\begin{array}{l}4.2 \mathrm{E}-04 \\
3.6 \mathrm{E}-02 \\
4.4 \mathrm{E}-02 \\
4.3 \mathrm{E}-01\end{array}$ & $\begin{array}{l}6.5 E-04 \\
6.0 E-02 \\
9.1 E-02 \\
6.9 E-01\end{array}$ & $\begin{array}{l}1.1 \mathrm{E}-04 \\
8.1 \mathrm{E}-03 \\
5.8 \mathrm{E}-03 \\
4.0 \mathrm{E}-02\end{array}$ & $\begin{array}{l}3.1 \mathrm{E}-06 \\
9.2 \mathrm{E}-02 \\
5.5 \mathrm{E}-02 \\
3.2 \mathrm{E}-01\end{array}$ & $\begin{array}{l}1.8 \mathrm{E}-03 \\
1.7 \mathrm{E}-01 \\
1.3 \mathrm{E}-01 \\
7.9 \mathrm{E}-01\end{array}$ \\
\hline TOTAL & $5.1 \mathrm{E}-01$ & $8.4 E-01$ & $5.4 E-02$ & 4.7E-01 & $1.1 \mathrm{E}+00$ \\
\hline
\end{tabular}

Dose Conmltments (person-rem) from Airborne Pathways

IotalBody Gl-LLI Ihyreld Bone Llver Lung

Infant

$1.9 \mathrm{E}-04 \quad 1.9 \mathrm{E}-04$

4.8E-03 $\quad 1.6 \mathrm{E}-04 \quad 2.1 \mathrm{E}-04$

$2.3 \mathrm{E}-04$

Child

2.2E-03

$2.2 \mathrm{E}-03$

2.8E-02

$1.7 \mathrm{E}-03$

$2.3 \mathrm{E}-03$

$2.8 \mathrm{E}-03$

Teen

$1.5 \mathrm{E}-03$

$1.5 \mathrm{E}-03$

$1.1 E-02$

$1.2 \mathrm{E}-03$

$1.6 \mathrm{E}-03$

$2.4 E-03$

Adult

8. $9 \mathrm{E}-03$

8. $8 \mathrm{E}-03$

$3.9 E-02$

$6.9 \mathrm{E}-03$

8.9E-03

$1.1 E-02$

TOTAL

$1.3 \mathrm{E}-02$

$1.3 \mathrm{E}-02$

8.3E-02

$9.9 \mathrm{E}-03 \quad 1.3 \mathrm{E}-02$

1.7E-02

Production/Consumption factors:

Produce: $<1$

Milk: <1

Meat: <1

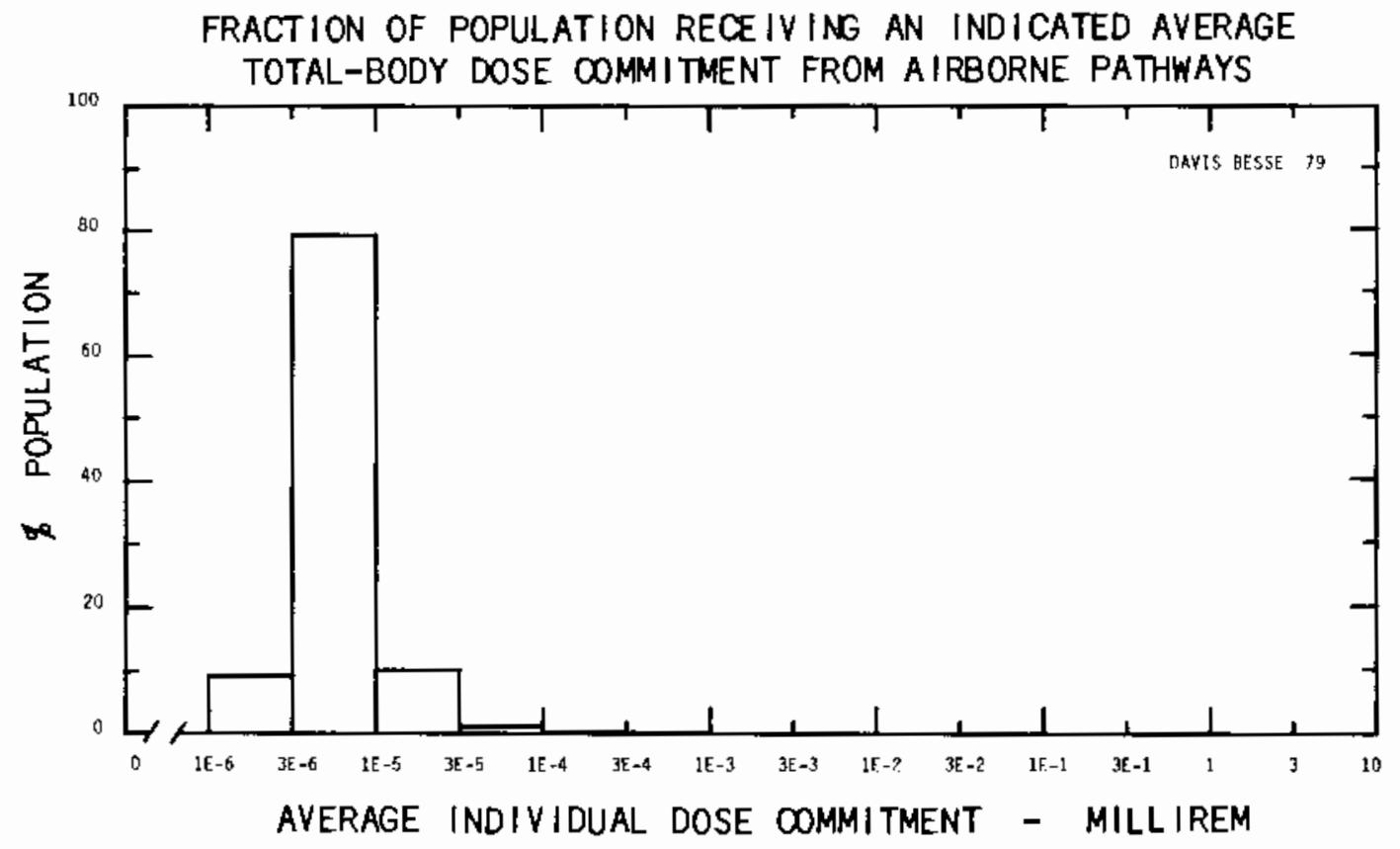


Ste: DRESDEN

GRUNDY COUNTY, ILLINOIS

Lecation: $\quad \mathrm{N} 41.3897^{\circ} \quad 88.2714^{\circ}$

POPULATION DATA

Total Population Within 20-to-80-km Region: 6.4E6

Major Metropolitan Centers within Region:

Center

Chicago SISA

Gary-Hz-mond-E.Ch I cago-SisA

Joll let

Aurora

EIgIn
Population

$7,100,000$ (a)

$640,000^{(a)}$

81,000

75,000

56,000
Location

$72 \mathrm{~km} \quad \mathrm{NE}$

$80 \mathrm{~km}$ ENE

$24 \mathrm{~km}$ NE

$43 \mathrm{~km} \mathrm{~N}$

$74 \mathrm{~km} \mathrm{~N}$

SITE SPECIFIC DATA - AIRBORNE PATHWAYS

Average Annual State Production

of Crops and Animal Products

In 80-km Radius Circle

Regional Productivity Factor:

AnImal GrazIng Factor:

Meteorology Perlod of Record: 1 JAN 74 - 31 JAN 75 Recovery: 778
Veg: 1.1E8 kilogram

Mllk: 1.8E8 Iiter

Meat: $1.9 E 8$ kilogram

1

0.5

SITE SPECIFIC DATA - WATERBORNE PATHWAYS VIO ILLINOIS RIVER

Average River Flow at Site: $12,000 \mathrm{ft}^{3} / \mathrm{s}$

Drinking Water:

Exposed Population: (b)

Fish:

Edible Harvest: (b) $\mathrm{kg} / \mathrm{yr}$

(a) Population of total SMSA given; population of SMSA fraction within $80 \mathrm{~km}$ of site would be somewhat smaller.

(b) River water used for sewage disposal for Chicago, so population doses from I iquid pathways assumed to be near zero. 
POPULATION DOSE-COMMITTMENT ESTIMATES AND AVERAGE INDIVIDUAL DOSE-COMMITMENT HISTOGRAM FOR DRESDEN 1, 2 ND 3

Dose Commitments (person-rem) from Liquid Pathways Iotal Body Gl-LLl Thyrold Bone Llver

Little or No Liquid Pathway Doses

\begin{tabular}{|c|c|c|c|c|c|}
\hline Iotal Body & Gl-LLل & Ihyrold & Bone & Llyer & Lung \\
\hline $\begin{array}{l}2.2 \mathrm{E}-01 \\
2.5 \mathrm{E}+00 \\
1.8 \mathrm{E}+00 \\
1.1 \mathrm{E}+01\end{array}$ & $\begin{array}{l}2.1 \mathrm{E}-01 \\
2.6 \mathrm{E}+00 \\
2.0 \mathrm{E}+00 \\
1.2 \mathrm{E}+01\end{array}$ & $\begin{array}{l}1.9 \mathrm{E}+00 \\
1.8 \mathrm{E}+01 \\
9.1 \mathrm{E}+00 \\
4.2 \mathrm{E}+01\end{array}$ & $\begin{array}{l}2.8 \mathrm{E}-01 \\
5.5 \mathrm{E}+00 \\
2.8 \mathrm{E}+00 \\
1.4 \mathrm{E}+01\end{array}$ & $\begin{array}{l}2.2 \mathrm{E}-01 \\
2.5 \mathrm{E}+00 \\
1.8 \mathrm{E}+00 \\
1.1 \mathrm{E}+01\end{array}$ & $\begin{array}{l}3.4 \mathrm{E}-01 \\
4.0 \mathrm{E}+00 \\
3.1 \mathrm{E}+00 \\
1.6 \mathrm{E}+01\end{array}$ \\
\hline $1.5 \mathrm{E}+01$ & $1.7 \mathrm{E}+01$ & $7.0 \mathrm{E}+01$ & $2.3 \mathrm{E}+01$ & $1.5 \mathrm{E}+01$ & $2.3 E+01$ \\
\hline
\end{tabular}

Production/Consumption factors:

Produce: $<1$

MIlk: <1

Meat: $<1$

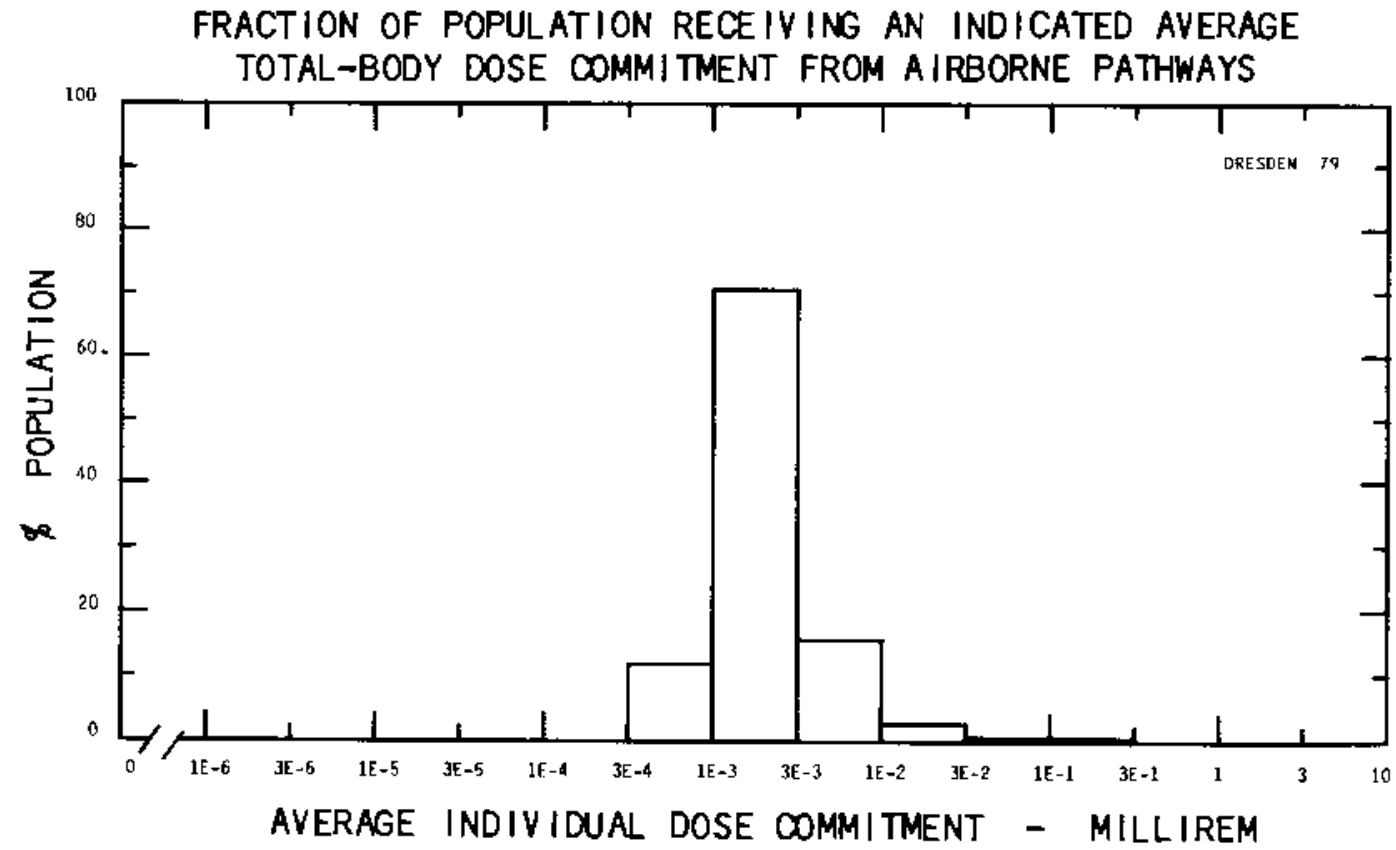


Slte: DUANE ARAOLD

Lecation: N $42.0981^{\circ}$
CEDAR RAPIDS, IOMA

W $91.7725^{\circ}$

POPULATION DATA

Total Population within 20-to-80-km Region: 5.7E

Major Metropolitan Centers Within Region: Center Population Location

Cedar Raplds

Waterioo

110,000

78,000

lowa City

48,000

$\begin{array}{ll}16 \mathrm{~km} & \text { SE } \\ 66 \mathrm{~km} & \mathrm{~N} \\ 53 \mathrm{~km} & \text { SSE }\end{array}$

SITE SPECIFIC DATA - AIRBORNE PATHWAYS

Average Annual State Production

of Crops and Animal Products

In 80-km Radius Circle

Reglonal Productivity Factor:

Animal Grazing Factor:

Meteorology Perlod of Record: 1 FEB 71 - 31 DEC 75 Recovery: 57\%
Veg: 9.8E kllogram

Milk: 2.6E8 liter

Meat: 4.2E8 kilogram

1

0.5

SITE SPECIFIC DATA - WATERBORNE PATHWAYS VIa CEDAR RIVER

$\begin{array}{ll} & \begin{array}{l}\text { Average River Flow } \\ \text { at Site: } 3,100 \mathrm{ft}^{3} / \mathrm{s}\end{array} \\ \text { Drinking Water: } & \text { Exposed Population: } 110,000 \\ & \text { Dilution Factor: } 1 \\ & \text { Edible Harvest: (a) } \\ \text { Dilution Factor: } 1\end{array}$

(a) No fish catch data was given in FES (1973); thus, population was assumed to eat $f$ ish at generic consumption rates (Table A-1). 


\section{POPULATION DOSE-COMMITMENT ESTIMATES AND AVERAGE INDIVIDUAL DOSE-COMMITMENT HISTOGRAM FOR \\ DUANE ARMOLD}

Infant

Child

Teen

Adult

TOTAL

\begin{tabular}{|c|c|c|c|c|c|c|}
\hline & Iotal Body & لـل| & Ihyroid & Bone & Liver & Lung \\
\hline $\begin{array}{l}\text { Infant } \\
\text { ChIld } \\
\text { Teen } \\
\text { Adult }\end{array}$ & $\begin{array}{l}6.9 \mathrm{E}-03 \\
7.7 \mathrm{E}-02 \\
5.6 \mathrm{E}-02 \\
3.4 \mathrm{E}-01\end{array}$ & $\begin{array}{l}6.9 \mathrm{E}-03 \\
7.7 \mathrm{E}-02 \\
5.7 \mathrm{E}-02 \\
3.4 \mathrm{E}-01\end{array}$ & $\begin{array}{l}2.1 \mathrm{E}-02 \\
2.4 \mathrm{E}-01 \\
1.5 \mathrm{E}-01 \\
7.2 \mathrm{E}-01\end{array}$ & $\begin{array}{l}6.9 \mathrm{E}-03 \\
7.7 \mathrm{E}-02 \\
5.6 \mathrm{E}-02 \\
3.4 \mathrm{E}-01\end{array}$ & $\begin{array}{l}7.0 \mathrm{E}-03 \\
7.8 \mathrm{E}-02 \\
5.7 \mathrm{E}-02 \\
3.4 \mathrm{E}-01\end{array}$ & $\begin{array}{l}7.1 \mathrm{E}-03 \\
8.0 \mathrm{E}-02 \\
6.1 \mathrm{E}-02 \\
3.5 \mathrm{E}-01\end{array}$ \\
\hline TOTAL & $4.8 E-01$ & $4.8 \mathrm{E}-01$ & $1.1 E+00$ & $4.8 \mathrm{E}-01$ & 4.9E-01 & $5.0 E-01$ \\
\hline
\end{tabular}

Production/Consumption factors:
Produce: $<1$
Milk: $\quad 3.4$
Meat: 9.1

FRACTION OF POPULATION RECEIVING AN INDICATED AVERAGE TOTAL-BODY DOSE COMMITMENT FROM AIRBORNE PATHWAYS

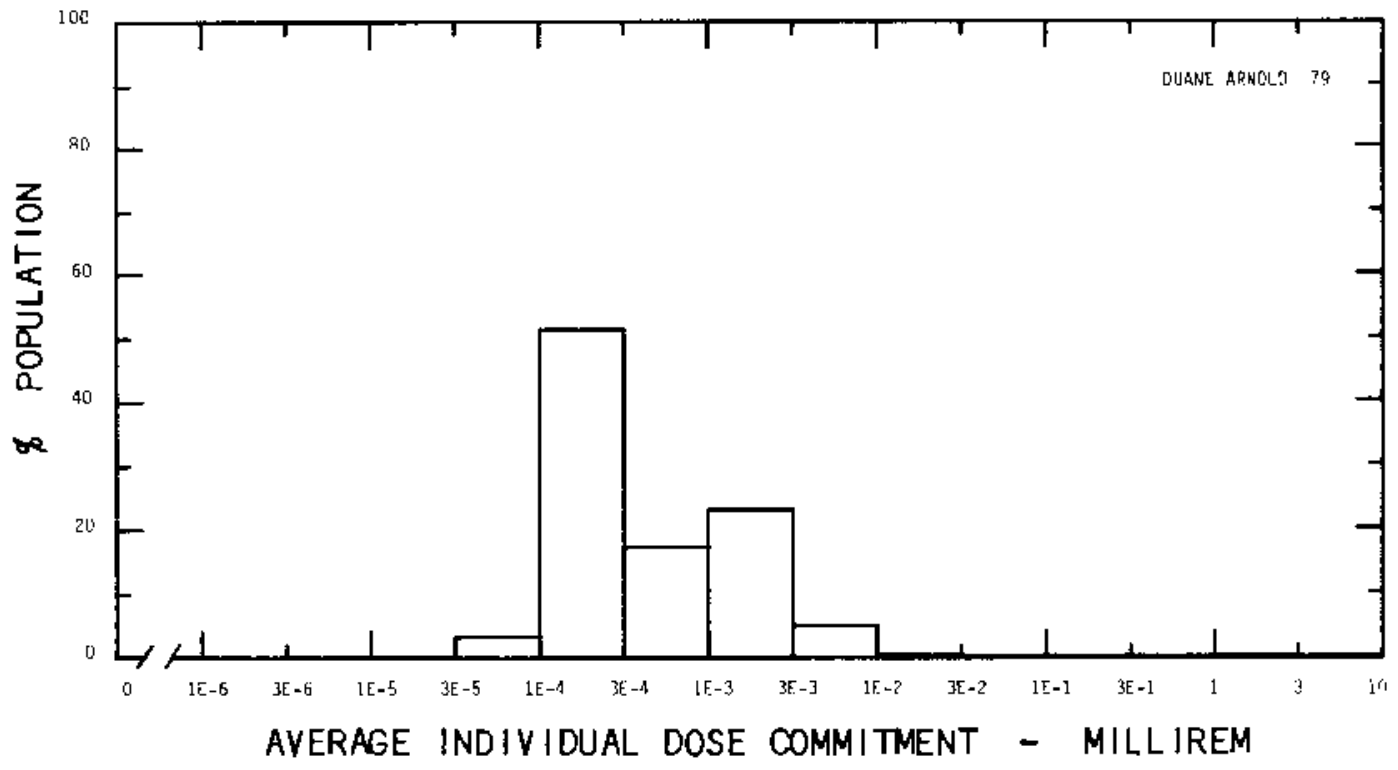


Slte: J. M. FARLEY

DOTHN, NABANA

Location: N $31.2233^{\circ} \quad$ N $85.3933^{\circ}$

POPULATION DATA

Total Population Within 20-to-80-km RegIon: 3.3E5

Major Metropolitan Centers Within Reglon:

Center

Dothan

Enterpr Iso

Ozark
Population

40,000

17,000

15,000
Location

$27 \mathrm{~km} \quad \mathrm{H}$

$72 \mathrm{~km} \quad \mathrm{H}$

$60 \mathrm{kn} \mathrm{N}$

SITE SPECIFIC DATA - AIRBORNE PATHNAYS

Average Annual State Production

of Crops and Animal Products

In 80-km Radius Circle

Reglonal Productivity Factor:

Animal Grazing Factor:

Meteorology Perlod of Record: 1 APR 71 - 31 MAR 75 Recovery: 100\$
Veg: 1.7E7 kilogram

Milk: 5.7E7 liter

Meat: 8.6E7 kllogram

0.95

0.8

SITE SPECIFIC DATA - WATERBORNE PATHWAYS VIa CHATTAHOOCHEE RIVER 


\section{POPULATION DOSE-COMMITMENT ESTIMATES AND AVERAGE INDIVIDUAL DOSE-COMMITMENT HISTOGRAM FOR \\ J. M. FARLEY}

Dose Commitments (person-rem) from Liquid Pathways

TotalBody GLLLl Thyreld Bone Llyer

$\begin{array}{llllll}\text { Infant } & 0.0 \mathrm{E}+00 & 0.0 \mathrm{E}+00 & 0.0 \mathrm{E}+00 & 0.0 \mathrm{E}+00 & 0.0 \mathrm{E}+00 \\ \text { Child } & 1.5 \mathrm{E}-04 & 4.5 \mathrm{E}-04 & 1.1 \mathrm{E}-04 & 4.9 \mathrm{E}-04 & 5.7 \mathrm{E}-04 \\ \text { Teen } & 2.2 \mathrm{E}-04 & 9.2 \mathrm{E}-04 & 8.1 \mathrm{E}-05 & 3.0 \mathrm{E}-04 & 4.8 \mathrm{E}-04 \\ \text { Adult } & 2.2 \mathrm{E}-03 & 8.0 \mathrm{E}-03 & 5.6 \mathrm{E}-04 & 1.7 \mathrm{E}-03 & 2.9 \mathrm{E}-03 \\ \text { TOTAL } & 2.5 \mathrm{E}-03 & 9.4 \mathrm{E}-03 & 7.5 \mathrm{E}-04 & 2.5 \mathrm{E}-03 & 4.0 \mathrm{E}-03\end{array}$

Dose Commitments (person-rem) from Alrborne Pathways

Ietal Body GL-LLل Ihyreld Bene Llyer Lung

$\begin{array}{lllllll}\text { Infant } & 2.7 \mathrm{E}-03 & 2.7 \mathrm{E}-03 & 2.7 \mathrm{E}-03 & 2.7 \mathrm{E}-03 & 2.7 \mathrm{E}-03 & 2.8 \mathrm{E}-03 \\ \text { Child } & 3.0 \mathrm{E}-02 & 3.0 \mathrm{E}-02 & 3.0 \mathrm{E}-02 & 3.1 \mathrm{E}-02 & 3.0 \mathrm{E}-02 & 3.2 \mathrm{E}-02 \\ \text { Teen } & 2.2 \mathrm{E}-02 & 2.2 \mathrm{E}-02 & 2.2 \mathrm{E}-02 & 2.2 \mathrm{E}-02 & 2.2 \mathrm{E}-02 & 2.4 \mathrm{E}-02 \\ \text { Adult } & 1.3 \mathrm{E}-01 & 1.3 \mathrm{E}-01 & 1.3 \mathrm{E}-01 & 1.3 \mathrm{E}-01 & 1.3 \mathrm{E}-01 & 1.4 \mathrm{E}-01 \\ \text { TOTAL } & 1.9 \mathrm{E}-01 & 1.9 \mathrm{E}-01 & 1.9 \mathrm{E}-01 & 1.9 \mathrm{E}-01 & 1.9 \mathrm{E}-01 & 2.0 \mathrm{E}-01\end{array}$

Production/Consumption factors:

Produce: <1 Milk: $1.3 \quad$ Meat: 3.1

FRACTION OF POPULATION RECEIVING AN INDICATED AVERAGE TOTAL-BODY DOSE COMMITMENT FROM AIRBORNE PATHWAYS

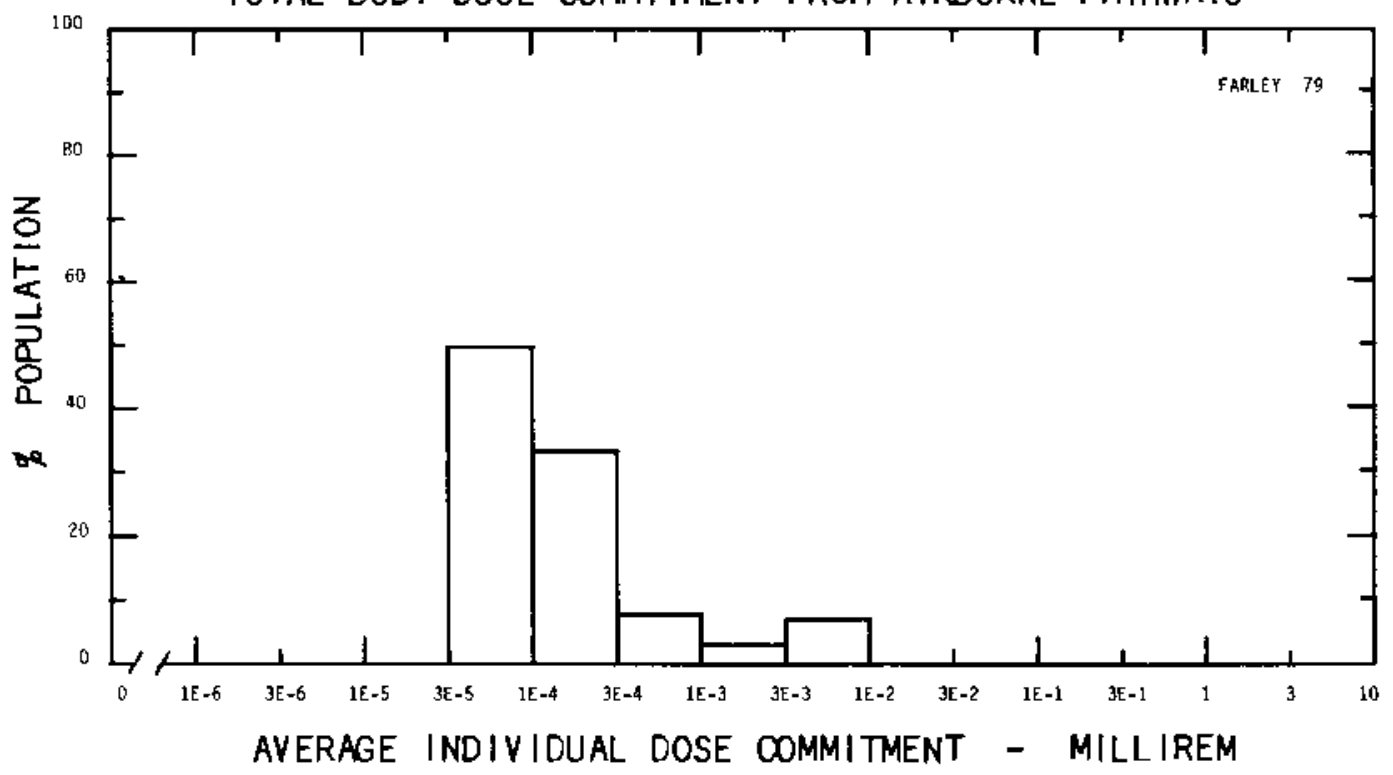


Site: J. A. FITZPATRICK

OSWEGO, NEH YORK

Location: N $43.5217^{\circ}$ W $76.3980^{\circ}$

POPULATION DATA

Total Population within 20-to-80-km Reglon: 8.1E5

Major Metropolitan Centers within Region:

Center.

Syracuse SMSA

Rome

Auburn
Population

620,000

48,000

33,000
Lecation

$54 \mathrm{~km}$ SSE

$80 \mathrm{~km}$ ESE

$66 \mathrm{~km}$ SSH

SITE SPECIFIC DATA - AIRBORNE PATHNAYS

Average Annual State Production

of Crops and AnImal Products

In 80-km Radius Circle

Reglonal Productivity Factor:

Animal Grazing Factor:

Meteorology Per Jod of Record: 1 JAN 74 - 31 DEC 75
Veg: $7.6 \mathrm{El}$ kilogram

Milk: 7.0E8 I Iter

Meat: $3.3 \mathrm{E} / \mathrm{kilogram}$

0.7

0.5

Recovery: $97 \%$

SITE SPECIFIC DATA - WATERBORNE PATHWAYS VIa LAKE ONTARIO

$\begin{array}{ll} & \begin{array}{l}\text { Average Dilution Flow } \\ \text { from Plant: } 560 \mathrm{ft} / \mathrm{s}\end{array} \\ \text { Drinking Water: } & \text { Exposed Population: } 530,000(\mathrm{a}) \\ & \text { Dilution Factor: } 0.003 \\ & \text { Edible Harvest: } 7.3 \mathrm{E}) \mathrm{kg} / \mathrm{yr} \\ & \text { Dilution Factor: } 0.005\end{array}$

(a) Population exposed to drinking water derived from Nine Mile Point FES (1974).

(b) Dilution factors derlved from FES (1973). 
POPULATION DOSE-COMMITMENT ESTIMATES AND

AVERAGE INDIVIDUAL DOSE-COMMITMENT HISTOGRAM FOR

\section{J. A. FITZPATRICK}

Dose Commitments (person-rem) from Liquid Pathways

Total Body Gl-LLl Thyrold Bone Llyer

$\begin{array}{llllll}\text { Infant } & 1.2 \mathrm{E}-04 & 7.2 \mathrm{E}-05 & 6.9 \mathrm{E}-05 & 5.3 \mathrm{E}-04 & 7.3 \mathrm{E}-04 \\ \text { Child } & 8.9 \mathrm{E}-03 & 2.0 \mathrm{E}-03 & 5.2 \mathrm{E}-04 & 4.1 \mathrm{E}-02 & 4.7 \mathrm{E}-02 \\ \text { Teen } & 1.5 \mathrm{E}-02 & 2.6 \mathrm{E}-03 & 1.7 \mathrm{E}-04 & 2.3 \mathrm{E}-02 & 3.7 \mathrm{E}-02 \\ \text { Adult } & 1.6 \mathrm{E}-01 & 2.4 \mathrm{E}-02 & 1.2 \mathrm{E}-03 & 1.3 \mathrm{E}-01 & 2.2 \mathrm{E}-01 \\ \text { TOTAL } & 1.8 \mathrm{E}-01 & 2.8 \mathrm{E}-02 & 1.9 \mathrm{E}-03 & 2.0 \mathrm{E}-01 & 3.0 \mathrm{E}-01\end{array}$

Dose Commitments (person-rem) from Airborne Pathways

TotalBody GJLLI Ihyrold Bone Llyer Lung

$\begin{array}{lllllll}\text { Infant } & 8.6 \mathrm{E}-04 & 8.5 \mathrm{E}-04 & 4.0 \mathrm{E}-03 & 8.7 \mathrm{E}-04 & 8.9 \mathrm{E}-04 & 9.0 \mathrm{E}-04 \\ \text { Child } & 9.7 \mathrm{E}-03 & 9.7 \mathrm{E}-03 & 3.1 \mathrm{E}-02 & 9.8 \mathrm{E}-03 & 9.8 \mathrm{E}-03 & 1.0 \mathrm{E}-02 \\ \text { Teen } & 7.0 \mathrm{E}-03 & 7.1 \mathrm{E}-03 & 1.5 \mathrm{E}-02 & 6.9 \mathrm{E}-03 & 7.1 \mathrm{E}-03 & 7.8 \mathrm{E}-03 \\ \text { Adult } & 4.2 \mathrm{E}-02 & 4.3 \mathrm{E}-02 & 6.8 \mathrm{E}-02 & 4.2 \mathrm{E}-02 & 4.2 \mathrm{E}-02 & 4.5 \mathrm{E}-02 \\ \text { TOTAL } & 6.0 \mathrm{E}-02 & 6.1 \mathrm{E}-02 & 1.2 \mathrm{E}-01 & 5.9 \mathrm{E}-02 & 6.0 \mathrm{E}-02 & 6.4 \mathrm{E}-02\end{array}$

Production/Consumption factors:

Produce: <1 Milk: $4.6 \quad$ Meat: <1

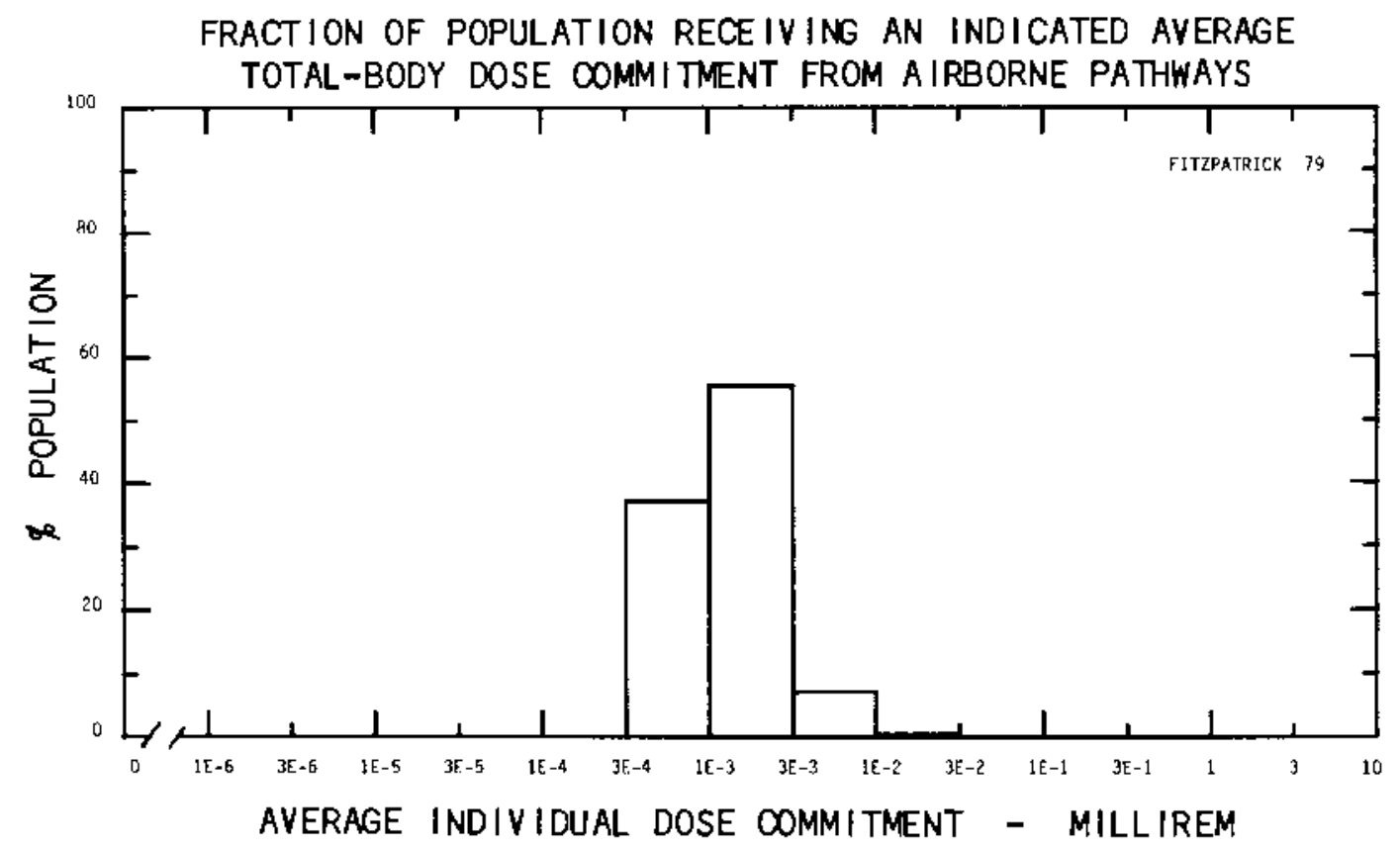


Site: FORT CNLHOW

Location: N $41.5200^{\circ}$
WASHINGTON COUNTY, MEBRASKA

W $96.0780^{\circ}$

POPULATION DATA
Total Population WIthin 20-to-80-km Reglon: $7.5 E 5$
Major Metropolitan Centers within Region:
Center
Population
Lecation
Omaha SISA
580,000
$32 \mathrm{~km} \quad$ SSE

SITE SPECIFIC DATA - AIRBORNE PATHWAYS

Average Annual State Production

of Crops and Anlmal Products

In 80-km Radius Circle

Reglonal Productivity Factor:

Animal Grazlng Factor:

Meteorology Perlod of Record: 1 JAN 74 - 31 DEC 74 Recovery: 98\%
Veg: 9.7E7 kilogram

Milk: 7.2E7 liter

Meat: $2.0 \mathrm{E} 8 \mathrm{kilogram}$

1

0.5

SITE SPECIFIC DATA - WATERBORNE PATHWAYS vIA MISSOURI RIVER

Average River FIow at Site: $27,000 \mathrm{ft}^{3} / \mathrm{s}$

Drinking water:

Exposed Population: $580,000^{(a)}$

Dilution Factor: 1

Edible Harvest: $1.064 \mathrm{~kg} / \mathrm{yr}$

Dilution Factor: 1

(a) Drinking water pollution assumed to be Omaha SMSA (FES, 1972). 


\begin{abstract}
POPULATION DOSE-COMMITMENT ESTIMATES AND AVERAGE INDIVIDUAL DOSE-COMMITMENT HISTOGRAM FOR
\end{abstract}

\title{
FORT CALHOWN
}

Dose Comm I tments (person-rem) from Liquid Pathways

Iotal Body

Gl-Lلl Thyroid

Bene

Lلer

Infant

$5.5 \mathrm{E}-03$

$5.2 \mathrm{E}-03$

$2.0 \mathrm{E}-02$

4.7E-03

$1.1 \mathrm{E}-02$

Child

$6.6 \mathrm{E}-02$

5.5E-02

1.6E-01

5. $2 \mathrm{E}-02$

1. $1 \mathrm{E}-01$

3. $0 \mathrm{E}-02$

2. 2E-02

$1.4 \mathrm{E}-02$

4. 4E-02

Adult

2.8E-01

$1.9 E-01$

3.9E-01

9.0E-02

$3.2 \mathrm{E}-01$

TOTAL

3. $8 \mathrm{E}-01$

2. $8 \mathrm{E}-01$

6.2E-01

$1.6 \mathrm{E}-01$

4. 9E-01

Dose Commitments (person-rem) from Alrborne Pathways

\section{Iotal Body Gl-LLL Ihyrold Bone Llver Lung}

$\begin{array}{lllllll}\text { Infant } & 1.0 \mathrm{E}-04 & 1.0 \mathrm{E}-04 & 5.8 \mathrm{E}-04 & 1.0 \mathrm{E}-04 & 1.0 \mathrm{E}-04 & 1.1 \mathrm{E}-04 \\ \text { Child } & 1.2 \mathrm{E}-03 & 1.2 \mathrm{E}-03 & 6.0 \mathrm{E}-03 & 1.4 \mathrm{E}-03 & 1.2 \mathrm{E}-03 & 1.3 \mathrm{E}-03 \\ \text { Teen } & 8.5 \mathrm{E}-04 & 8.5 \mathrm{E}-04 & 2.7 \mathrm{E}-03 & 8.9 \mathrm{E}-04 & 8.5 \mathrm{E}-04 & 1.0 \mathrm{E}-03 \\ \text { Adult } & 5.1 \mathrm{E}-03 & 5.1 \mathrm{E}-03 & 1.2 \mathrm{E}-02 & 5.2 \mathrm{E}-03 & 5.1 \mathrm{E}-03 & 5.6 \mathrm{E}-03 \\ \text { TOTAL } & 7.2 \mathrm{E}-03 & 7.2 \mathrm{E}-03 & 2.1 \mathrm{E}-02 & 7.5 \mathrm{E}-03 & 7.2 \mathrm{E}-03 & 8.0 \mathrm{E}-03\end{array}$

Production/Consumption factors:

Produce: $<1$ Milk: $<1$ Meat: 3.3

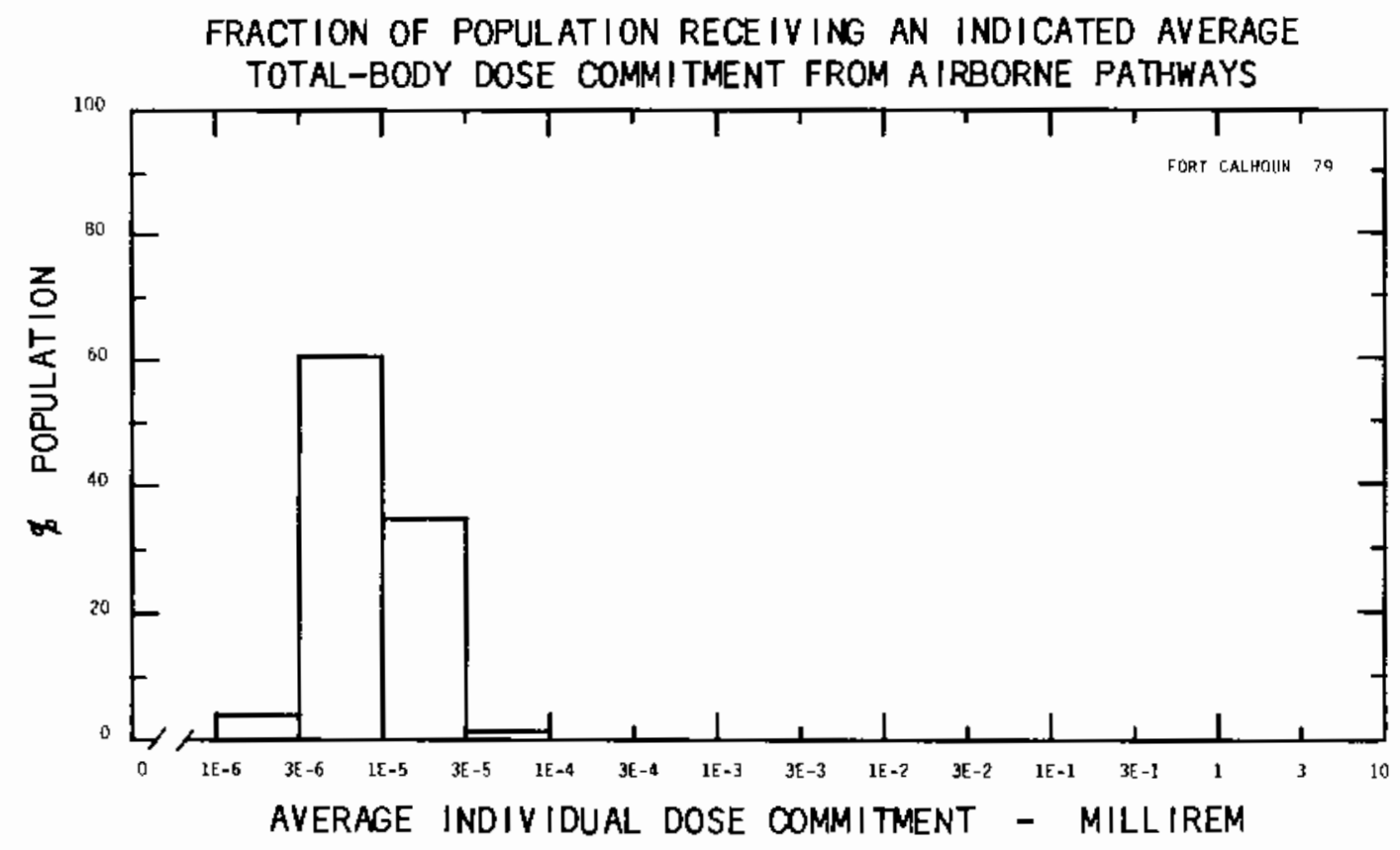


SIte: R. E. GINM

ONTARIO, NEM YORK

Lecation: $\quad \mathrm{N} 43.2772^{\circ} \quad W 77.3081^{\circ}$

POPULATION DATA

Total Population within 20-to-80-km Region: 1.1E6

Major Metropolitan Centers within Region:

\begin{tabular}{lcccc}
\multicolumn{1}{c}{ Center } & Population & \multicolumn{2}{c}{ Lecation } \\
Rochester SMSA & 940,000 & $34 \mathrm{~km}$ & WSW \\
Auburn & 34,000 & $64 \mathrm{~km}$ & SE \\
Oswego & 23,000 & $56 \mathrm{~km}$ & ENE
\end{tabular}

SITE SPECIFIC DATA - AIRBORNE PATHWAYS

Average Annual State Production

of Crops and Animal Products

In 80-km Radius Circle

Regional Productivity Factor:

AnImal Grazing Factor:

Meteorology Period of Record: 1 JAN 66 - 31 DEC 67 Recovery: 89\%
Veg: 7.6E7 kilogran

MIIk: 7.0EB liter

Meat: 3.3E7 kilogram

0.6

0.5

SITE SPECIFIC DATA - WATERBORNE PATHWAYS VIa LAKE ONTARIO

Average Dilution Flow

from Plant: $1100 \mathrm{ft}^{3} / \mathrm{s}$

OrInkIng Water:

Exposed Population: 530,000

Dilution Factor: $\mathbf{0 . 0 1}$

FIsh:

Edible Harvest: $7.3 \mathrm{E5} \mathrm{kg} / \mathrm{yr}$

Dilution Factor: 0.01 (

(a) Dilution factors from FES (1973). 
POPULATION DOSE-COMMITMENT ESTIMATES AND

AVERAGE INDIVIDUAL DOSE-COMMITMENT HISTOGRAM FOR

\section{R. E. GINM}

Dose Comm I tments (person-rem) from Liquid Pathways

Iotal Body

Gl-LLI

Thyrold

Bone

Luer

Infant

Child

Teen

Adult

$9.7 \mathrm{E}-04$

9.6E-04

$2.5 \mathrm{E}-03$

$1 \cdot 1 \mathrm{E}-04$

2.1E-02

$5.5 \mathrm{E}-03$

1. 2E-02

$1.1 \mathrm{E}-02$

$7.4 \mathrm{E}-03$

2. $9 \mathrm{E}-03$

5.7E-03

4.7E-03

5. 8E-02

$1.7 \mathrm{E}-02$

1. 1E-03

5. 3E-02

4. 1E-02

8.9E-02

2.5E-02

1. $6 \mathrm{E}-02$

$8.4 \mathrm{E}-03$

7. $1 \mathrm{E}-02$

$5.8 \mathrm{E}-02$

$2.5 E-02$

6.1E-02

TOTAL

Dose Commitments (person-rem) from Airborne Pathways

TotalBody GL-LLl Thyrold Bone Llver Lung

$\begin{array}{lllllll}\text { Infant } & 5.3 \mathrm{E}-04 & 5.2 \mathrm{E}-04 & 5.6 \mathrm{E}-03 & 1.8 \mathrm{E}-04 & 5.4 \mathrm{E}-04 & 5.3 \mathrm{E}-04 \\ \text { Child } & 6.7 \mathrm{E}-03 & 6.7 \mathrm{E}-03 & 3.8 \mathrm{E}-02 & 2.0 \mathrm{E}-03 & 6.8 \mathrm{E}-03 & 6.9 \mathrm{E}-03 \\ \text { Teen } & 4.3 \mathrm{E}-03 & 4.3 \mathrm{E}-03 & 1.6 \mathrm{E}-02 & 1.4 \mathrm{E}-03 & 4.3 \mathrm{E}-03 & 4.6 \mathrm{E}-03 \\ \text { Adult } & 2.4 \mathrm{E}-02 & 2.4 \mathrm{E}-02 & 6.3 \mathrm{E}-02 & 8.5 \mathrm{E}-03 & 2.4 \mathrm{E}-02 & 2.4 \mathrm{E}-02 \\ \text { TOTAL } & 3.5 \mathrm{E}-02 & 3.5 \mathrm{E}-02 & 1.2 \mathrm{E}-01 & 1.2 \mathrm{E}-02 & 3.5 \mathrm{E}-02 & 3.6 \mathrm{E}-02\end{array}$

Production/Consumption factors:

Produce: <1 MIIk: $2.8 \quad$ Meat: $<1$

FRACTION OF POPULATION RECEIVING AN INDICATED AVERAGE TOTAL-BODY DOSE COMMITMENT FROM AIRBORNE PATHWAYS

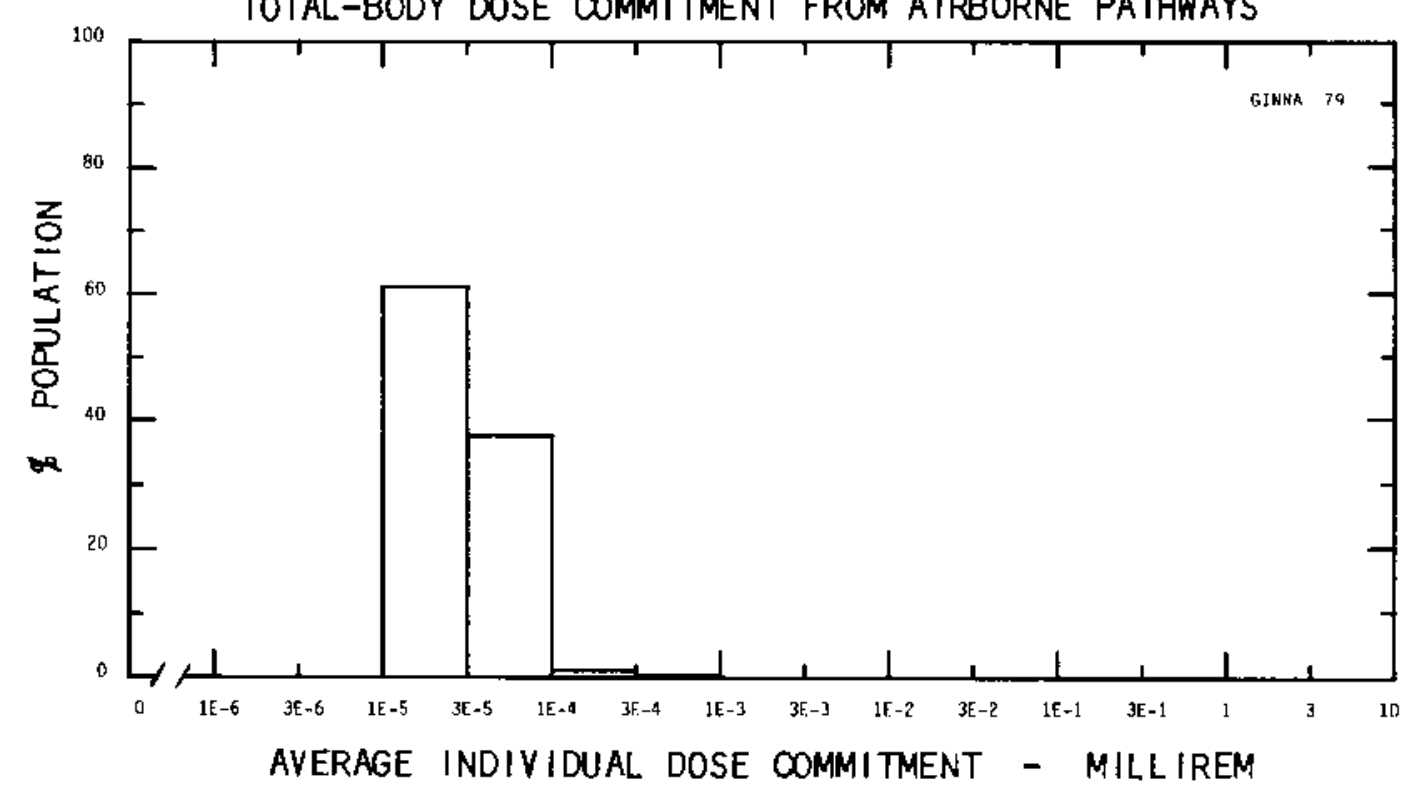


SIte: HNDN NECK (CONA. YANEE)

Location: N $41.4822^{\circ}$ W $72.4989^{\circ}$

POPULATION DATA

Total Population within 20-to-80-km Reglon: 3.4E6

Major Metropolitan Centers With In Region:

Center

Hartford SMSA

Ner Haven SUSA

Springtield SUSA

Waterbury
Population

840,000

770,000

600,000

110,000
Location

$37 \mathrm{~km} \quad \mathrm{M}$

$40 \mathrm{~km} \quad \mathrm{NSW}$

$72 \mathrm{~km} \quad \mathrm{~N}$

$47 \mathrm{~km} \quad \mathrm{H}$

SITE SPECIFIC DATA - AIRBORNE PATHWAYS

Average Annual State Production

of Crops and AnImal Products

In 80-km Radius Circle

Regional Productivity Factor:

Animal Grazing Factor:

Meteorology Perlod of Record: 1 JAN 75 - 31 DEC 75
Veg: $3.2 E 7$ kilogram

Milk: 4.4E8 liter

Meat: 2.0E7 kJlogram

0.7

0.6

SITE SPECIFIC DATA - WATERBORNE PATHWAYS VIa DISCHARGE CANAL TO OONN. RIVER

Average DIscharge Canal

Flow at Site: $770 \mathrm{ft}^{3} / \mathrm{s}$

Drinking Water:

Exposed Population: None

Fish:

Edible Harvest: $9.1 \mathbf{B}^{(\mathrm{a})} \mathrm{kg} / \mathrm{yr}$

Dilution Factor: 1

(a) Caught in discharge canal (FES, 1973). 


\section{POPULATION DOSE-COMMITMENI ESTIMATES AND AVERAGE INDIVIDUAL DOSE-COMMITMENT HISTOGRAM FOR HADDN NECK}

Dose Commitments (person-rem) from Liquid Pathways

Iotal_Body

Gl-LLI

Ihyreds

Bone

Luer

Infant

Child

Teen

Adult

TOTAL

$\begin{array}{lllll}0.0 \mathrm{E}+00 & 0.0 \mathrm{E}+00 & 0.0 \mathrm{E}+00 & 0.0 \mathrm{E}+00 & 0.0 \mathrm{E}+00 \\ 9.8 \mathrm{E}-03 & 2.5 \mathrm{E}-02 & 3.0 \mathrm{E}-03 & 4.2 \mathrm{E}-02 & 5.0 \mathrm{E}-02 \\ 1.8 \mathrm{E}-02 & 5.2 \mathrm{E}-02 & 2.3 \mathrm{E}-03 & 2.6 \mathrm{E}-02 & 4.2 \mathrm{E}-02 \\ 1.9 \mathrm{E}-01 & 4.5 \mathrm{E}-01 & 1.6 \mathrm{E}-02 & 1.5 \mathrm{E}-01 & 2.5 \mathrm{E}-01 \\ 2.2 \mathrm{E}-01 & 5.3 \mathrm{E}-01 & 2.1 \mathrm{E}-02 & 2.2 \mathrm{E}-01 & 3.5 \mathrm{E}-01\end{array}$

Dose Commitments (person-rem) from Alrborne Pathways

Iotal Body Gل-LLل Ihyreld Bene Liver Lung

$\begin{array}{lllllll}\text { Infant } & 1.9 \mathrm{E}-01 & 1.9 \mathrm{E}-01 & 2.7 \mathrm{E}-01 & 8.7 \mathrm{E}-01 & 2.0 \mathrm{E}-01 & 2.0 \mathrm{E}-01 \\ \text { ChIld } & 1.3 \mathrm{E}+00 & 1.3 \mathrm{E}+00 & 1.7 \mathrm{E}+00 & 5.8 \mathrm{E}+00 & 1.3 \mathrm{E}+00 & 1.3 \mathrm{E}+00 \\ \text { Teen } & 4.4 \mathrm{E}-01 & 4.4 \mathrm{E}-01 & 6.3 \mathrm{E}-01 & 1.8 \mathrm{E}+00 & 4.4 \mathrm{E}-01 & 4.5 \mathrm{E}-01 \\ \text { Adult } & 1.5 \mathrm{E}+00 & 1.5 \mathrm{E}+00 & 2.2 \mathrm{E}+00 & 5.2 \mathrm{E}+00 & 1.5 \mathrm{E}+00 & 1.5 \mathrm{E}+00 \\ \text { TOTAL } & 3.4 \mathrm{E}+00 & 3.4 \mathrm{E}+00 & 4.8 \mathrm{E}+00 & 1.4 \mathrm{E}+01 & 3.4 \mathrm{E}+00 & 3.5 \mathrm{E}+00\end{array}$

Production/Consumption factors:

Produce: $<1 \quad$ Milk: $<1 \quad$ Meat: $<1$

FRACTION OF POPULATION RECEIVING AN INDICATED AVERAGE

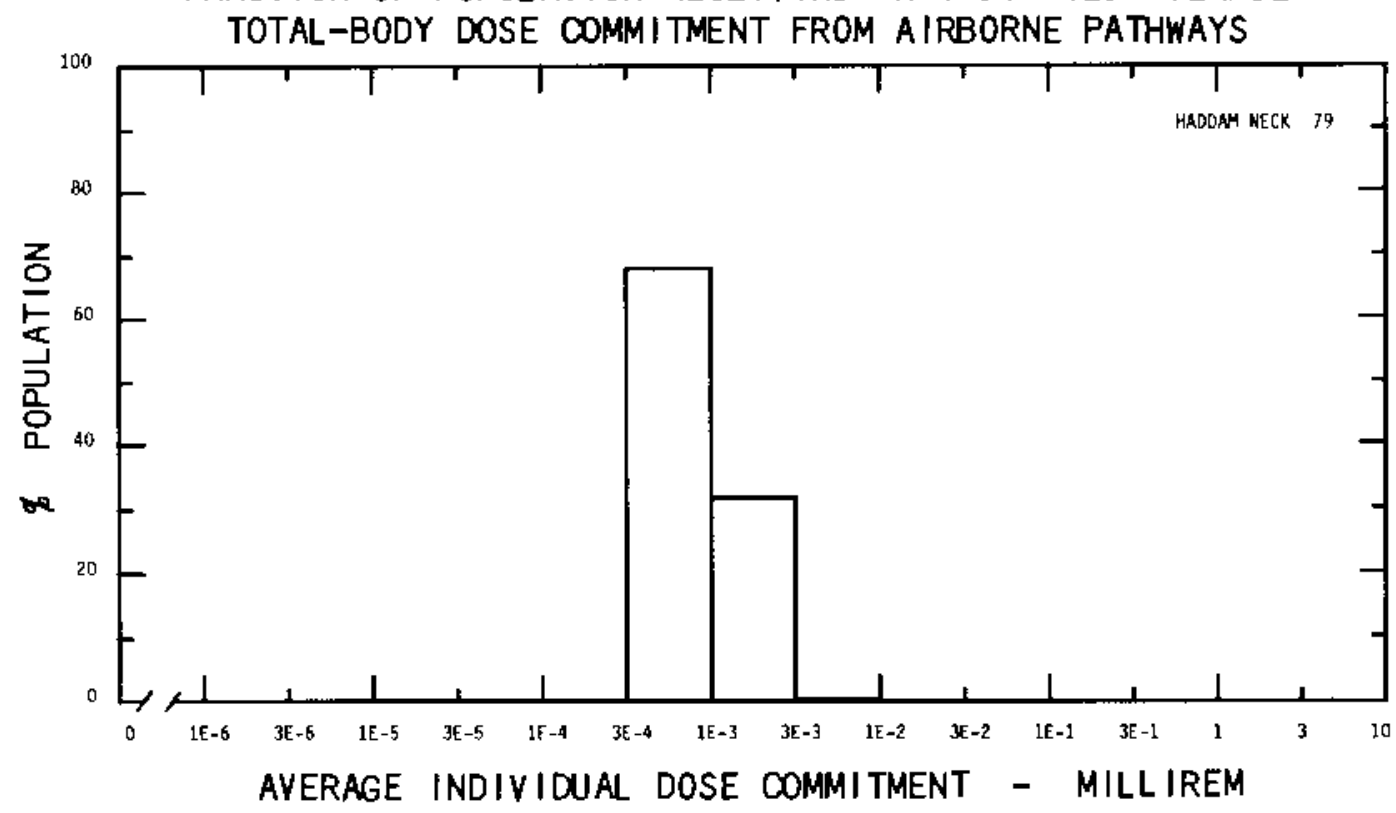


Location: N $31.9344^{\circ} \quad$ W $82.3444^{\circ}$

POPULATION DATA

Total Population Within 20-to-80-km Region: 2.855

Major Metropolitan Centers Within Region:

Center Population Location

$\begin{array}{llll}\text { Waycross } & 21,000 & 80 \mathrm{~km} & \mathrm{~S}\end{array}$

SITE SPECIFIC DATA - AIRBORNE PATHNAYS

Average Annual State Production

of Crops and AnImal Products

In 80-km Radius Circle

Reglonal Productivity Factor:

Animal Grazing Factor:

Meteorology Perlod of Record: 1 JUN 70 - 31 ANG 74 Recovery: 87\%
Veg: 8.8E6 kilogram

MIlk: 7.0E7 liter

Meat: 8.1E7 kllogram

1

0.8

SITE SPECIFIC DATA - WATERBORNE PATHWAYS VIa ALTAMAHA RIVER

Drinking Water:

Fish:
Average River Flow

at Site: $13,000 \mathrm{ft}^{3} / \mathrm{s}$

Exposed Population: None

Edible Harvest: $6.3 \mathrm{EF}^{(\mathrm{a})} \mathrm{kg} / \mathrm{yr}$ DIlution Factor: 1

(a) Commercial catch plus 3 pounds of game fish per year taken from rlver by average person (FES, 1972). 


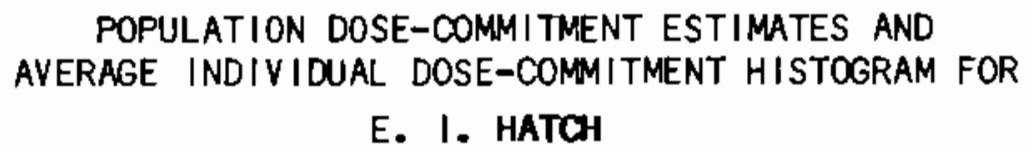

Dose Commitments (person-rem) from Liquid Pathways

Total Body

Gل-LلL

Ihyrold

Bene

Luer

Infant

ChIId

$0.0 \mathrm{E}+00$

$0.0 \mathrm{E}+00$

$0.0 \mathrm{E}+00$

$0.0 \mathrm{E}+00$

$0.0 \mathrm{E}+00$

Teen

Adult

6.0E-03

6.7 E-04

$7.4 \mathrm{E}-04$

2.2E-02

2.8E-02

$1.0 \mathrm{E}-02$

$1.4 \mathrm{E}-03$

5. $4 \mathrm{E}-04$

$1.4 \mathrm{E}-02$

$2.4 \mathrm{E}-02$

$1.0 \mathrm{E}-01$

$1.3 \mathrm{E}-02$

3. $5 \mathrm{E}-03$

7.9E-02

1. $4 \mathrm{E}-01$

TOTAL

1.2E-01

1.5E-02

$4.8 \mathrm{E}-03$

$1.2 \mathrm{E}-01$

2.0E-01

Dose Commitments (person-rem) from Airborne Pathways IotalBody Gl-LLل Thyrold Bone Liver Lung

$\begin{array}{lllllll}\text { Infant } & 1.7 \mathrm{E}-04 & 1.6 \mathrm{E}-04 & 6.5 \mathrm{E}-03 & 1.7 \mathrm{E}-04 & 2.0 \mathrm{E}-04 & 1.8 \mathrm{E}-04 \\ \text { ChIld } & 2.0 \mathrm{E}-03 & 1.9 \mathrm{E}-03 & 3.7 \mathrm{E}-02 & 1.8 \mathrm{E}-03 & 2.1 \mathrm{E}-03 & 2.1 \mathrm{E}-03 \\ \text { Teen } & 1.4 \mathrm{E}-03 & 1.4 \mathrm{E}-03 & 1.5 \mathrm{E}-02 & 1.2 \mathrm{E}-03 & 1.4 \mathrm{E}-03 & 1.6 \mathrm{E}-03 \\ \text { Adult } & 8.2 \mathrm{E}-03 & 8.1 \mathrm{E}-03 & 5.3 \mathrm{E}-02 & 7.2 \mathrm{E}-03 & 8.3 \mathrm{E}-03 & 8.8 \mathrm{E}-03 \\ \text { TOTAL } & 1.2 \mathrm{E}-02 & 1.2 \mathrm{E}-02 & 1.1 \mathrm{E}-01 & 1.0 \mathrm{E}-02 & 1.2 \mathrm{E}-02 & 1.3 \mathrm{E}-02\end{array}$

Production/Consumption factors:

Produce: <1 Milk: 1.9 Meat: 3.6

FRACTION OF POPULATION RECEIVING AN INDICATED AVERAGE TOTAL-BODY DOSE COMMITMENT FROM AIRBORNE PATHWAYS

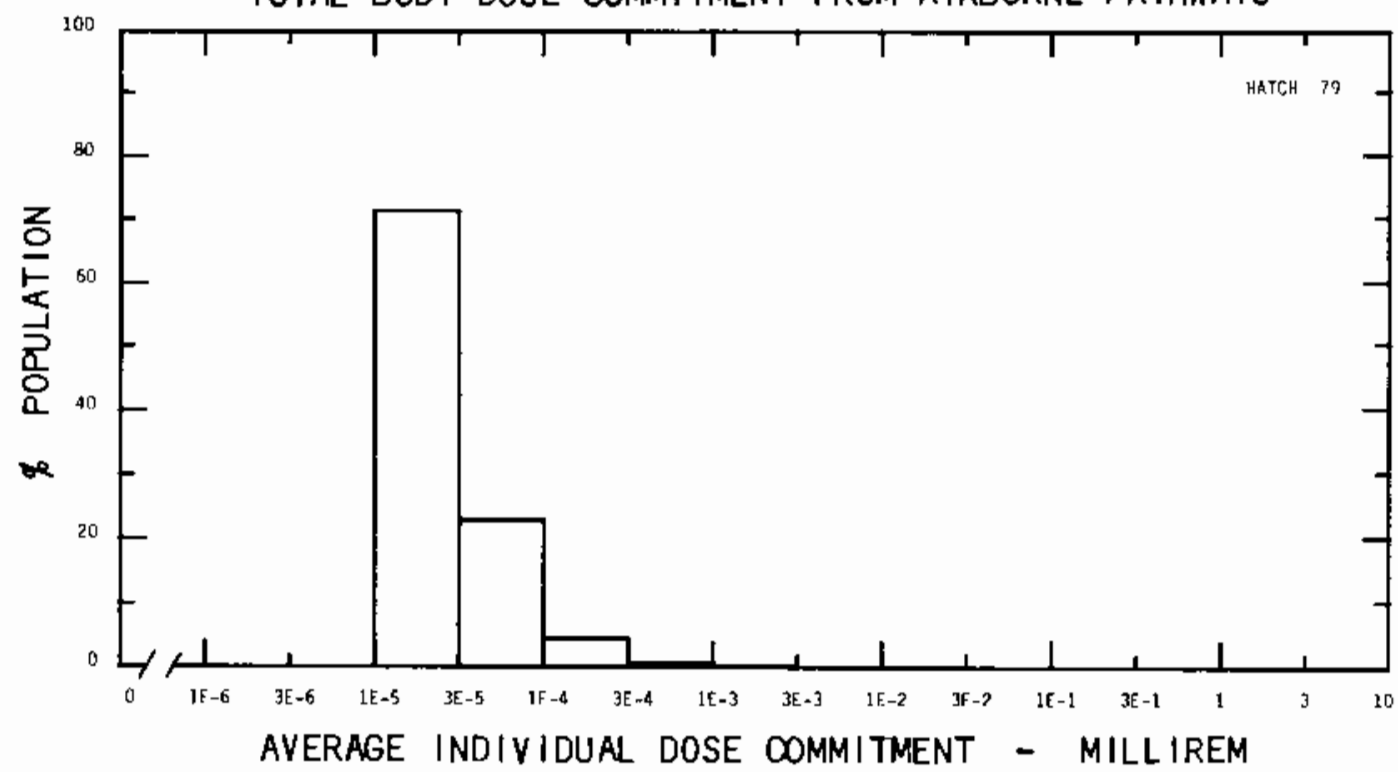


SIte: HUMOLDT BAY

Location:

POPULATION DATA

Total Population Within 20-to-80-km Reglon: 1.1E5

Major Metropolitan Centers Within Region:

Center

Population

Lecation

Eureka

28,000

$6.4 \mathrm{~km} \mathrm{~N}$

SITE SPECIFIC DATA - AIRBORNE PATHWAYS

Average Annual State Production

Of Crops and Animal Products

In 80-km Radius Circle

Regional Productivity Factor:

Animal Grazing Factor:
Veg: 4.8E7 kilogram

MIIk: 2.3E8 liter

Meat: 5.0E7 kllogram

0.5

1

Meteorology Perlod of Record: 1 JN 66 - 31 DEC 67 Recovery: 96\%

SITE SPECIFIC DATA - WATERBORNE PATHWAYS VIa HUMBOLDT BAY

Fish:

Invertebrates:
Average Dilution Flow

from Plant: $110 \mathrm{tt}^{3} / \mathrm{s}$

Edible Harvest: $44^{(a)} \mathrm{kg} / \mathrm{yr}$

Dilution Factor: 0.001

Edible Harvest: $5 \mathrm{Es}^{(\mathrm{a})} \mathrm{kg} / \mathrm{yr}$

Dllution Factor: 0.002

(a) Edible seafood harvest taken from Noshkin ot al., 1976. 


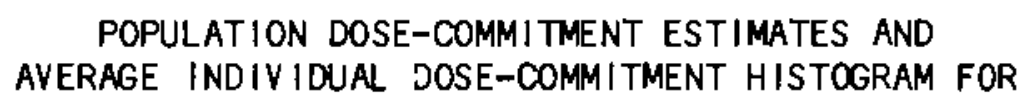

HUMBODT BAY

Dose Commltments (person-rem) from Liquid Pathways

Total Body Gl-LL Thyrodd Bone Llver

$\begin{array}{llllll}\text { Infant } & 0.0 \mathrm{E}+00 & 0.0 \mathrm{E}+00 & 0.0 \mathrm{E}+00 & 0.0 \mathrm{E}+00 & 0.0 \mathrm{E}+00 \\ \text { Child } & 5.9 \mathrm{E}-06 & 5.0 \mathrm{E}-06 & 2.3 \mathrm{E}-10 & 2.6 \mathrm{E}-05 & 2.6 \mathrm{E}-05 \\ \text { Teen } & 8.9 \mathrm{E}-06 & 1.0 \mathrm{E}-05 & 2.0 \mathrm{E}-10 & 1.5 \mathrm{E}-05 & 2.2 \mathrm{E}-05 \\ \text { Adult } & 9.1 \mathrm{E}-05 & 8.6 \mathrm{E}-05 & 1.6 \mathrm{E}-09 & 8.9 \mathrm{E}-05 & 1.3 \mathrm{E}-04 \\ \text { TOTAL } & 1.1 \mathrm{E}-04 & 1.0 \mathrm{E}-04 & 2.1 \mathrm{E}-09 & 1.3 \mathrm{E}-04 & 1.8 \mathrm{E}-04\end{array}$

Dose Commltments (person-rem) from Airborne Pathways

IotalBody Gl-LLI Thyrodd Bone Liver Lung

$\begin{array}{lllllll}\text { Infant } & 5.1 \mathrm{E}-07 & 4.6 \mathrm{E}-07 & 4.5 \mathrm{E}-07 & 9.9 \mathrm{E}-07 & 4.9 \mathrm{E}-07 & 2.4 \mathrm{E}-06 \\ \text { ChIId } & 9.7 \mathrm{E}-06 & 8.2 \mathrm{E}-06 & 7.6 \mathrm{E}-06 & 2.5 \mathrm{E}-05 & 8.3 \mathrm{E}-06 & 4.0 \mathrm{E}-05 \\ \text { Teen } & 6.9 \mathrm{E}-06 & 6.5 \mathrm{E}-06 & 5.5 \mathrm{E}-06 & 1.9 \mathrm{E}-05 & 5.9 \mathrm{E}-06 & 3.5 \mathrm{E}-05 \\ \text { AduIt } & 4.0 \mathrm{E}-05 & 3.9 \mathrm{E}-05 & 3.2 \mathrm{E}-05 & 1.0 \mathrm{E}-04 & 3.4 \mathrm{E}-05 & 1.5 \mathrm{E}-04 \\ \text { TOTAL } & 5.7 \mathrm{E}-05 & 5.4 \mathrm{E}-05 & 4.5 \mathrm{E}-05 & 1.5 \mathrm{E}-04 & 4.9 \mathrm{E}-05 & 2.3 \mathrm{E}-04\end{array}$

Production/Consumption factors:

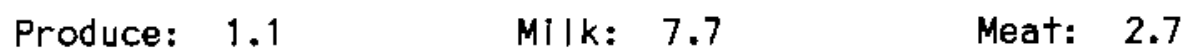

FRACTION OF POPULATION RECEIVING AN INDICATED AVERAGE TOTAL-BODY DOSE COMMITMENT FROM AIRBORNE PATHWAYS

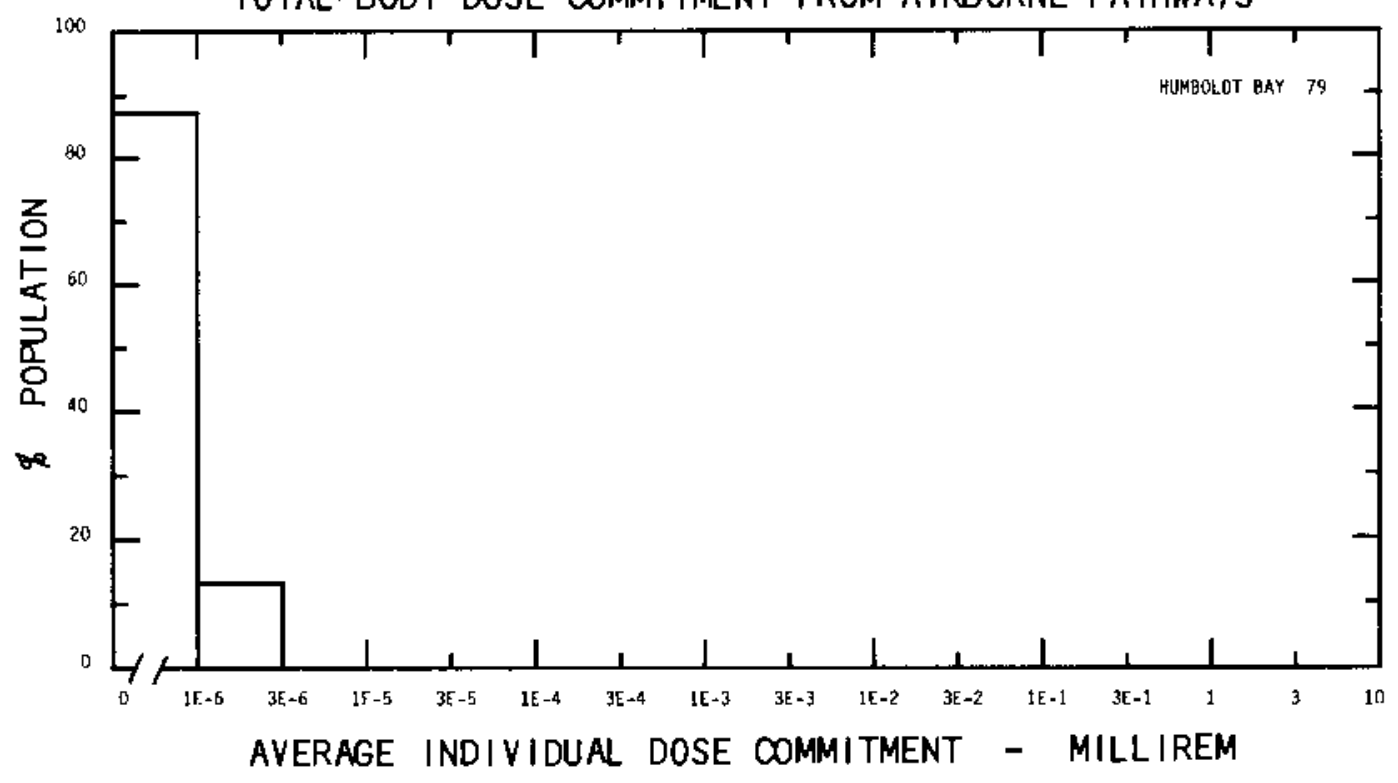


SIte: INDIN POINT

Lecation:

N $41.2656^{\circ}$

BUCHANAK, NEM YORK

W $73.9350^{\circ}$

POPLLATION DATA

Total Population Within 20-to-80-km Reglon: 1.6E7

Major Metropolitan Centers Within Region:

Center
Now York SMSA
Nowark SMSA
Stanford
Norvalk
Bridgeport
Poughkeepsie
White PIalns

Population

Location

Now York SMSA

Nowark SMSA

Stanford

Horralk

Poughkeepsie

White PIalns

$60 \mathrm{~km} \mathrm{~S}$

$64 \mathrm{~km}$ SSW

$42 \mathrm{~km} \quad S E$

$48 \mathrm{~km}$ ESE

$64 \mathrm{~km} \mathrm{E}$

$48 \mathrm{~km} \quad \mathrm{~N}$

$30 \mathrm{~km}$ SSE

SITE SPECIFIC DATA - AIRBORNE PATHWAYS

Average Annual State Production

of Crops and Animal Products

In 80-km Radius Circle

Reglonal Productivity Factor:

Animal Grazing Factor:

Meteorology Perlod of Record: 1 JN 75 - 31 DEC 75 Recovery: 96\%
Veg: 7.6E7 kilogram

Milk: 7.0EB liter

Meat: 3.3E7 kilogram

0.8

0.5

SITE SPECIFIC DATA - WATERBORNE PATHWAYS VIa HUDSON RIVER

$\begin{array}{ll} & \begin{array}{l}\text { Average River Flow } \\ \text { at Site: } 20,000 \mathrm{ft} / \mathrm{s}\end{array} \\ \text { Drinking Water: } & \text { Exposed Population: None } \\ \text { Fish: } & \text { Edible Harvest: (a) } \\ & \text { Dilution Factor: } 0.001(b)\end{array}$

(a) No fish catch data given in FES, so generic consumption rates used (Table A-1).

(b) One percent of population obtain $10 \%$ of their fish from river (FES, 1972). 


\begin{abstract}
POPULATION DOSE-COMMITMENT ESTIMATES AND AVERAGE INDIVIDUAL DOSE-COMMITMENT HISTOGRAM FOR
\end{abstract}

INDINN POINT 1, 2 AND 3

Dose Comml ments (person-rem) from Liquid Pathways

Total Body Gl-LLl Ihyrold Bone Liver

$\begin{array}{llllll}\text { Infant } & 0.0 \mathrm{E}+00 & 0.0 \mathrm{E}+00 & 0.0 \mathrm{E}+00 & 0.0 \mathrm{E}+00 & 0.0 \mathrm{E}+00 \\ \text { Child } & 2.1 \mathrm{E}-02 & 1.2 \mathrm{E}-03 & 2.0 \mathrm{E}-03 & 1.1 \mathrm{E}-01 & 1.2 \mathrm{E}-01 \\ \text { Teen } & 3.9 \mathrm{E}-02 & 2.3 \mathrm{E}-03 & 1.5 \mathrm{E}-03 & 6.8 \mathrm{E}-02 & 1.0 \mathrm{E}-01 \\ \text { Aduit } & 4.2 \mathrm{E}-01 & 1.9 \mathrm{E}-02 & 9.7 \mathrm{E}-03 & 4.0 \mathrm{E}-01 & 6.0 \mathrm{E}-01 \\ \text { TOTAL } & 4.8 \mathrm{E}-01 & 2.3 \mathrm{E}-02 & 1.3 \mathrm{E}-02 & 5.8 \mathrm{E}-01 & 8.2 \mathrm{E}-01\end{array}$

Dose Commitments (person-rem) from Alrborne Pathways Ietalbody Gl-LLl Thyrold Bone Llver Lung

$\begin{array}{lllllll}\text { Infant } & 7.5 \mathrm{E}-02 & 7.3 \mathrm{E}-02 & 3.8 \mathrm{E}-01 & 9.6 \mathrm{E}-02 & 1.0 \mathrm{E}-01 & 9.6 \mathrm{E}-02 \\ \text { Child } & 8.5 \mathrm{E}-01 & 8.2 \mathrm{E}-01 & 3.0 \mathrm{E}+00 & 1.0 \mathrm{E}+00 & 1.0 \mathrm{E}+00 & 1.2 \mathrm{E}+00 \\ \text { Teen } & 6.3 \mathrm{E}-01 & 6.0 \mathrm{E}-01 & 1.7 \mathrm{E}+00 & 6.6 \mathrm{E}-01 & 7.0 \mathrm{E}-01 & 9.4 \mathrm{E}-01 \\ \text { Adult } & 3.8 \mathrm{E}+00 & 3.7 \mathrm{E}+00 & 8.2 \mathrm{E}+00 & 3.8 \mathrm{E}+00 & 3.9 \mathrm{E}+00 & 4.9 \mathrm{E}+00 \\ \text { TOTAL } & 5.4 \mathrm{E}+00 & 5.1 \mathrm{E}+00 & 1.3 \mathrm{E}+01 & 5.6 \mathrm{E}+00 & 5.7 \mathrm{E}+00 & 7.1 \mathrm{E}+00\end{array}$

Production/Consumption factors:

Produce: $<1 \quad$ Milk: <1 Meat: <1

FRACTION OF POPULATION RECEIVING AN INDICATED AVERAGE TOTAL-BODY DOSE COMMITMENT FROM AIRBORNE PATHWAYS

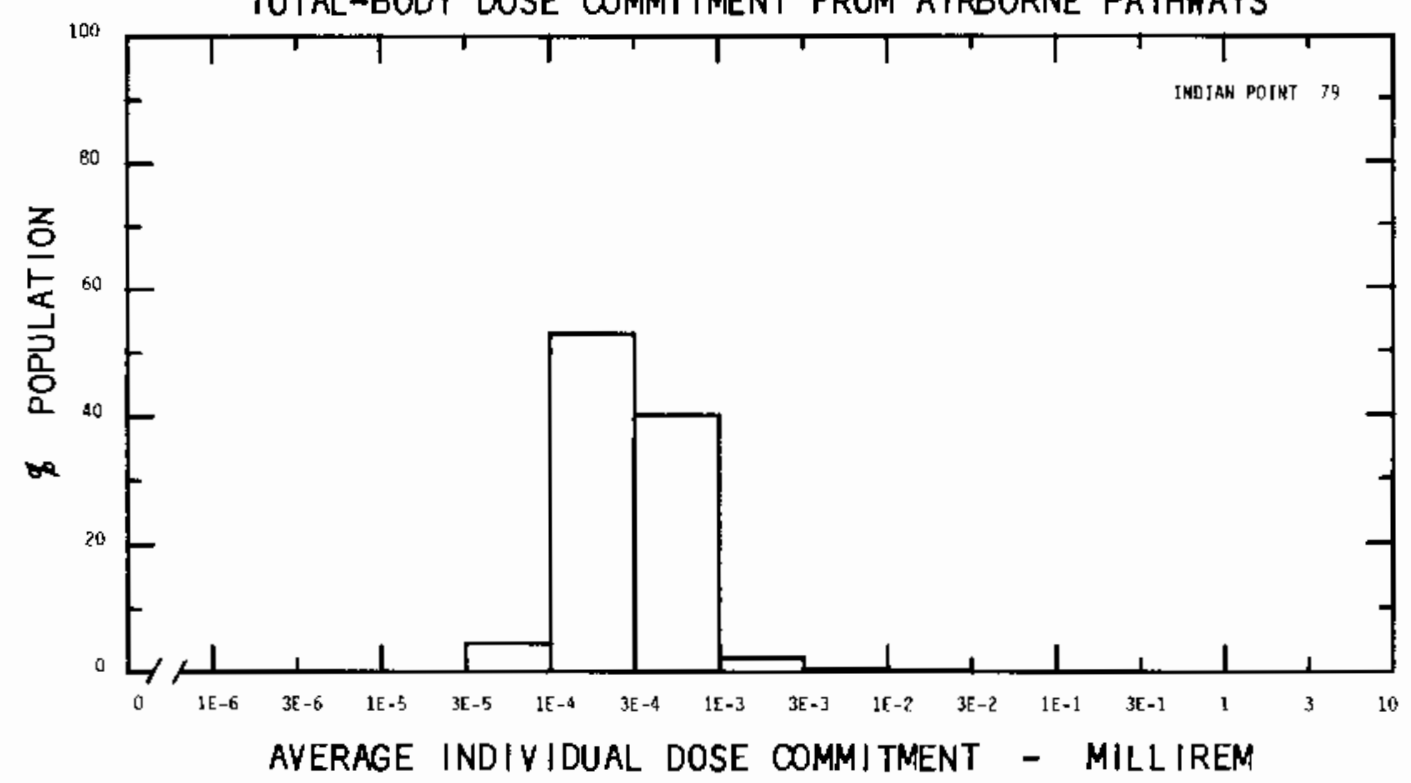


Slte: KENAUNEE

CNRLTON, WISCONSIN

Location:

N $44.3433^{\circ}$

$87.5350^{\circ}$

POPULATION DATA

Total Population within 20-to-80-km Region: 6.1E

$$
\text { Major Metropolitan Centers within Region: }
$$

Center

Sheboygan

Man Itowoc

Greenbay

Appleton
Population

52,000

36,000

94,000

61,000
Location

$64 \mathrm{~km} \quad S S H$

$30 \mathrm{kn}$ SSH

$40 \mathrm{kn} \quad W$

$72 \mathrm{~km} \quad$ WSW

SITE SPECIFIC DATA - AIRBORNE PATHWAYS

Average Annual State Production

Of Crops and Animal Products

In 80-km Radius Circle

Regional Productivity Factor:

Animal Grazing Factor:

Meteorology Perlod of Record: 2 JAN 69 - 31 DEC 69 Recovery: $76 \%$

SITE SPECIFIC DATA - WATERBORNE PATHWAYS VIa LAKE MICHIGAN

Average Dilution Flow from

Plant: $140 \mathrm{ft}^{3} / \mathrm{s}$

Drinking Water:

Exposed Population: 200,000

Dilution Factor: $8.2 \mathrm{E}-3$ (a)

Fish:

Edible Harvest: 1.1 (b) $\mathrm{kg} / \mathrm{yr}$

(a) Drinking water dilution factor estimated by averaging dilution factors derlved from FES (1972) suitably weighted for populations.

(b) Average individual consumption rate as given in the FES (1972) used in lieu of catch data.

(c) Dilution factor reduced $1 / 10$ from that used in FES in consideration of lake mixing. 


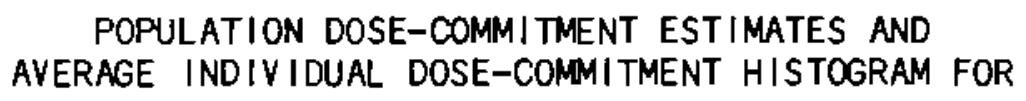

KEMAUNEE

Dose Commitments (person-rem) from Liquid Pathways

Iotal Body Gl-LLl Ihyrold Bone Liver

$\begin{array}{llllll}\text { Infant } & 2.9 \mathrm{E}-03 & 2.8 \mathrm{E}-03 & 2.6 \mathrm{E}-03 & 2.5 \mathrm{E}-03 & 5.8 \mathrm{E}-03 \\ \text { Child } & 1.0 \mathrm{E}-01 & 4.3 \mathrm{E}-02 & 2.8 \mathrm{E}-02 & 3.2 \mathrm{E}-01 & 4.2 \mathrm{E}-01 \\ \text { Teen } & 1.4 \mathrm{E}-01 & 3.4 \mathrm{E}-02 & 1.1 \mathrm{E}-02 & 1.8 \mathrm{E}-01 & 3.3 \mathrm{E}-01 \\ \text { Adult } & 1.5 \mathrm{E}+00 & 3.0 \mathrm{E}-01 & 9.2 \mathrm{E}-02 & 1.1 \mathrm{E}+00 & 2.0 \mathrm{E}+00 \\ \text { TOTAL } & 1.8 \mathrm{E}+00 & 3.8 \mathrm{E}-01 & 1.3 \mathrm{E}-01 & 1.6 \mathrm{E}+00 & 2.8 \mathrm{E}+00\end{array}$

Dose Commitments (person-rem) from Alrborne Pathways

Iotal Body Gl-LلL Ihyrold Bone Llver Lung

$\begin{array}{lllllll}\text { Infant } & 4.5 \mathrm{E}-05 & 4.5 \mathrm{E}-05 & 1.2 \mathrm{E}-04 & 2.0 \mathrm{E}-05 & 4.6 \mathrm{E}-05 & 4.7 \mathrm{E}-05 \\ \text { ChIld } & 6.3 \mathrm{E}-04 & 6.3 \mathrm{E}-04 & 1.2 \mathrm{E}-03 & 2.3 \mathrm{E}-04 & 6.3 \mathrm{E}-04 & 6.6 \mathrm{E}-04 \\ \text { Teen } & 4.1 \mathrm{E}-04 & 4.1 \mathrm{E}-04 & 6.5 \mathrm{E}-04 & 1.7 \mathrm{E}-04 & 4.1 \mathrm{E}-04 & 4.4 \mathrm{E}-04 \\ \text { Adult } & 2.3 \mathrm{E}-03 & 2.3 \mathrm{E}-03 & 3.3 \mathrm{E}-03 & 9.9 \mathrm{E}-04 & 2.3 \mathrm{E}-03 & 2.4 \mathrm{E}-03 \\ \text { TOTAL } & 3.4 \mathrm{E}-03 & 3.4 \mathrm{E}-03 & 5.2 \mathrm{E}-03 & 1.4 \mathrm{E}-03 & 3.4 \mathrm{E}-03 & 3.6 \mathrm{E}-03\end{array}$

Production/Consumption factors:

Produce: $<1$ Milk: 7.3 Meat: 1.0

FRACTION OF POPULATION RECEIVING AN INDICATED AVERAGE TOTAL-BODY DOSE COMMITMENT FROM AIRBORNE PATHWAYS

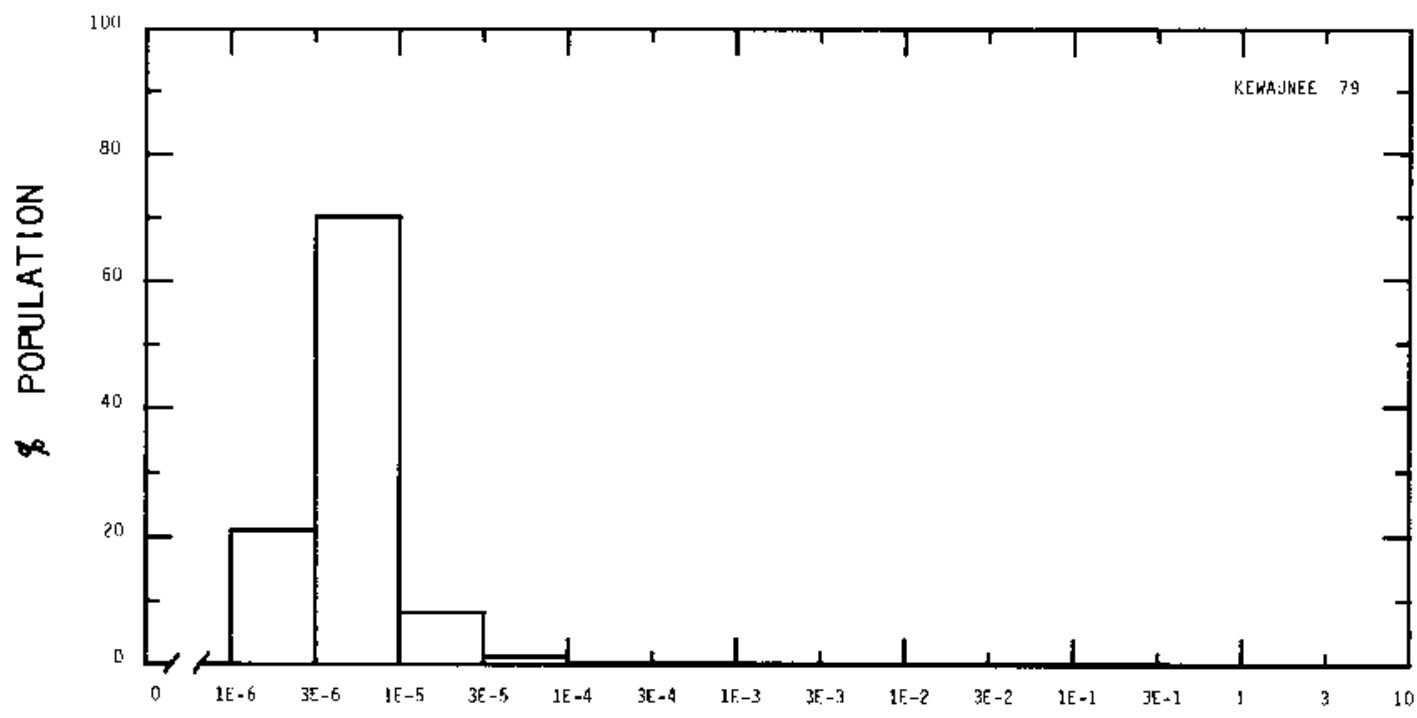

AVERAGE INDIVIDUAL DOSE COMMITMENT - MILLIREM 
SIte: LA CROSSE

Location:

POPULATION DATA

Total Population Within 20-to-80-km Region: 3.4E5

Major Metropol itan Centers within Region:

Center

La Crosse
Population

55,000
Lecation

$32 \mathrm{~km} \mathrm{~N}$

SITE SPECIFIC DATA - AIRBORNE PATHWAYS

Average Annual State Production

of Crops and Animal Products

In 80-km Radius Circle

Reglonal Productivity Factor:

AnImal Grazing Factor:
Veg: 7.2E7 kilogram

Milk: 1.2E9 I iter

Meat: $1.0 \mathrm{~EB}$ kilogram

1

0.5

Meteorology Perlod of Record: 1 JAN 75 - 31 DEC 75 Recovery: 97\%

SITE SPECIFIC DATA - WATERBORNE PATHWAYS VIa MISSISSIPPI RIVER

Average River

Flow at Site: $28,000 \mathrm{ft}^{3} / \mathrm{s}$

Drinking Water:

Exposed Population: None

Fish:

Edible Harvest: (a) $\mathrm{kg} / \mathrm{yr}$
Dilution Factor: $0.5 \mathrm{al}$

(a) No fish catch data given in FES, so $1 / 2$ population assumed to consume river $f$ Ish at generlc consumption rates (Table A-1). 


\section{POPULATION DOSE-COMMITMENT ESTIMATES AND AVERAGE INDIVIDUAL DOSE-COMMITMENT HISTOGRAM FOR}

\section{LA CROSSE}

Dose Cormitments (person-rem) from Liquid Pathways

Iotal Body Thyrold Bone Llyer

$\begin{array}{llllll}\text { Infant } & 0.0 \mathrm{E}+00 & 0.0 \mathrm{E}+00 & 0.0 \mathrm{E}+00 & 0.0 \mathrm{E}+00 & 0.0 \mathrm{E}+00 \\ \text { Child } & 1.6 \mathrm{E}-01 & 1.7 \mathrm{E}-01 & 5.3 \mathrm{E}-03 & 9.0 \mathrm{E}-01 & 9.2 \mathrm{E}-01 \\ \text { Teen } & 3.0 \mathrm{E}-01 & 3.6 \mathrm{E}-01 & 3.8 \mathrm{E}-03 & 5.4 \mathrm{E}-01 & 7.7 \mathrm{E}-01 \\ \text { Adult } & 3.2 \mathrm{E}+00 & 3.1 \mathrm{E}+00 & 2.5 \mathrm{E}-02 & 3.1 \mathrm{E}+00 & 4.6 \mathrm{E}+00 \\ \text { TOTAL } & 3.7 \mathrm{E}+00 & 3.7 \mathrm{E}+00 & 3.4 \mathrm{E}-02 & 4.6 \mathrm{E}+00 & 6.3 \mathrm{E}+00\end{array}$

Dose Commltments (person-rem) from Alrborne Pathways

Iotal Body Gl-LL Thyrold Bone Liver Lung

$\begin{array}{lllllll}\text { Infant } & 6.2 \mathrm{E}-03 & 6.2 \mathrm{E}-03 & 8.0 \mathrm{E}-03 & 6.7 \mathrm{E}-03 & 6.2 \mathrm{E}-03 & 6.6 \mathrm{E}-03 \\ \text { Child } & 7.1 \mathrm{E}-02 & 7.2 \mathrm{E}-02 & 8.8 \mathrm{E}-02 & 1.2 \mathrm{E}-01 & 6.9 \mathrm{E}-02 & 7.4 \mathrm{E}-02 \\ \text { Teen } & 5.1 \mathrm{E}-02 & 5.3 \mathrm{E}-02 & 5.8 \mathrm{E}-02 & 6.7 \mathrm{E}-02 & 5.1 \mathrm{E}-02 & 5.6 \mathrm{E}-02 \\ \text { Adult } & 3.1 \mathrm{E}-01 & 3.2 \mathrm{E}-01 & 3.4 \mathrm{E}-01 & 3.6 \mathrm{E}-01 & 3.1 \mathrm{E}-01 & 3.2 \mathrm{E}-01 \\ \text { TOTAL } & 4.4 \mathrm{E}-01 & 4.5 \mathrm{E}-01 & 4.9 \mathrm{E}-01 & 5.6 \mathrm{E}-01 & 4.3 \mathrm{E}-01 & 4.6 \mathrm{E}-01\end{array}$

Production/Consumption factors:

Produce: $1.1 \quad$ Milk: 27 Meat: 3.8

FRACTION OF POPULATION RECEIVING AN INDICATED AVERAGE

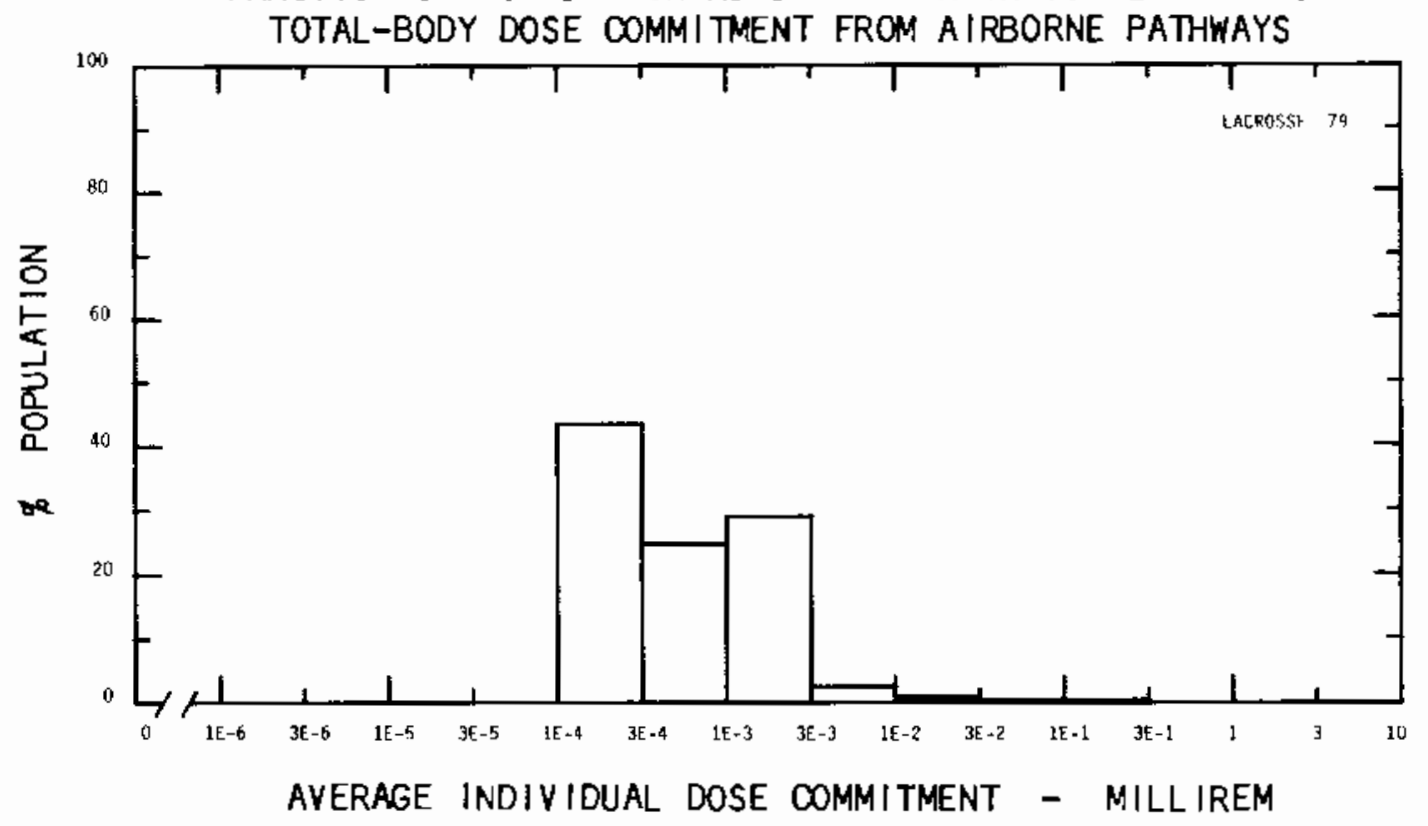


SIte: MAINE YANKEE

Location: N $43.9503^{\circ}$
LINCOLN COUNTY, MAINE

W $69.6964^{\circ}$

POPULATION DATA

Total Population Within 20-to-80-km Region: 5.7E5

Major Metropolitan Centers WithIn Reglon:

Center

Portl and

Leriston
Population

72,000

46,000
Lecation

$51 \mathrm{~km}$ SW

$40 \mathrm{~km} \quad \mathrm{~min}$

SITE SPECIFIC DATA - AIRBORNE PATHWAYS

Average Annual State Production

Of Crops and Anlmal Products

In 80-km Radius Circle

Regional Productivity Factor:

Animal Grazlng Factor:

Meteorology Period of Record: 1 APR 75 - 31 MAR 76 Recovery: 98\%
Veg: 2.4E8 kilogram

Milk: 6.6El liter

Meat: 4.3E6 kilogram

0.6

0.5

SITE SPECIFIC DATA - WATERBORNE PATHWAYS VIa ATLANTIC OCEAN
Average Dilution Flow
from Plant: $844 \mathrm{ft} / \mathrm{s}$
Fish:
Edible Harvest: (a) $\mathrm{kg} / \mathrm{yr}$
Dilution Factor: 0.001
Invertebrates:
Edible Harvest: (a) $\mathrm{kg} / \mathrm{yr}$
Dilution Factor: 0.002
(a) No seafood harvest data glven in FES (1972), thus generic population consumption rates used (Table A-1).




\section{POPULATION DOSE-COMMITMENT ESTIMATES AND AVERAGE INDIVIDUAL DOSE-COMMITMENT HISTOGRAM FOR MAINE YAMEE}

Dose Commitments (person-rem) from L lquid Pathways

Iotal Body

GI-LLI

Ihyrods Bone

Liver

Infant

$0.0 \mathrm{E}+00$

$0.0 E+00$

$0.0 \mathrm{E}+00$

$0.0 \mathrm{E}+00$

$0.0 \mathrm{E}+00$

Child

$6.8 \mathrm{E}-05$

4.8E-05

1. $0 \mathrm{E}-02$

1. $5 \mathrm{E}-04$

$1.7 \mathrm{E}-04$

$7.5 \mathrm{E}-05$

8.3E-05

$7.3 \mathrm{E}-03$

9.0E-05

$1.4 \mathrm{E}-04$

Adult

6.7E-04

7.3E-04

4. 8E-02

5. $2 \mathrm{E}-04$

8. $9 \mathrm{E}-04$

TOTAL

8.1E-04

8.6E-04

$6.6 \mathrm{E}-02$

$7.6 \mathrm{E}-04$

1.2E-03

Dose Commitments (person-rem) from Alrborne Pathways

Iotal Body Gل-LL Ihyrold Bone Liver Lun

$\begin{array}{lllllll}\text { Infant } & 5.7 \mathrm{E}-04 & 5.6 \mathrm{E}-04 & 9.8 \mathrm{E}-03 & 5.8 \mathrm{E}-04 & 6.0 \mathrm{E}-04 & 6.1 \mathrm{E}-04 \\ \text { Child } & 6.7 \mathrm{E}-03 & 6.5 \mathrm{E}-03 & 1.4 \mathrm{E}-01 & 6.7 \mathrm{E}-03 & 7.0 \mathrm{E}-03 & 7.1 \mathrm{E}-03 \\ \text { Teen } & 4.8 \mathrm{E}-03 & 4.7 \mathrm{E}-03 & 6.2 \mathrm{E}-02 & 4.7 \mathrm{E}-03 & 4.9 \mathrm{E}-03 & 5.6 \mathrm{E}-03 \\ \text { Adult } & 2.9 \mathrm{E}-02 & 2.8 \mathrm{E}-02 & 2.6 \mathrm{E}-01 & 2.8 \mathrm{E}-02 & 2.9 \mathrm{E}-02 & 3.1 \mathrm{E}-02 \\ \text { TOTAL } & 4.1 \mathrm{E}-02 & 4.0 \mathrm{E}-02 & 4.7 \mathrm{E}-01 & 4.0 \mathrm{E}-02 & 4.1 \mathrm{E}-02 & 4.4 \mathrm{E}-02\end{array}$

Production/Consumption factors:

Produce: 1.3

Milk: $<1$

Meat: <i

FRACTION OF POPULATION RECEIVING AN INDICATED AVERAGE TOTAL-BODY DOSE COMMITMENT FROM AIRBORNE PATHWAYS

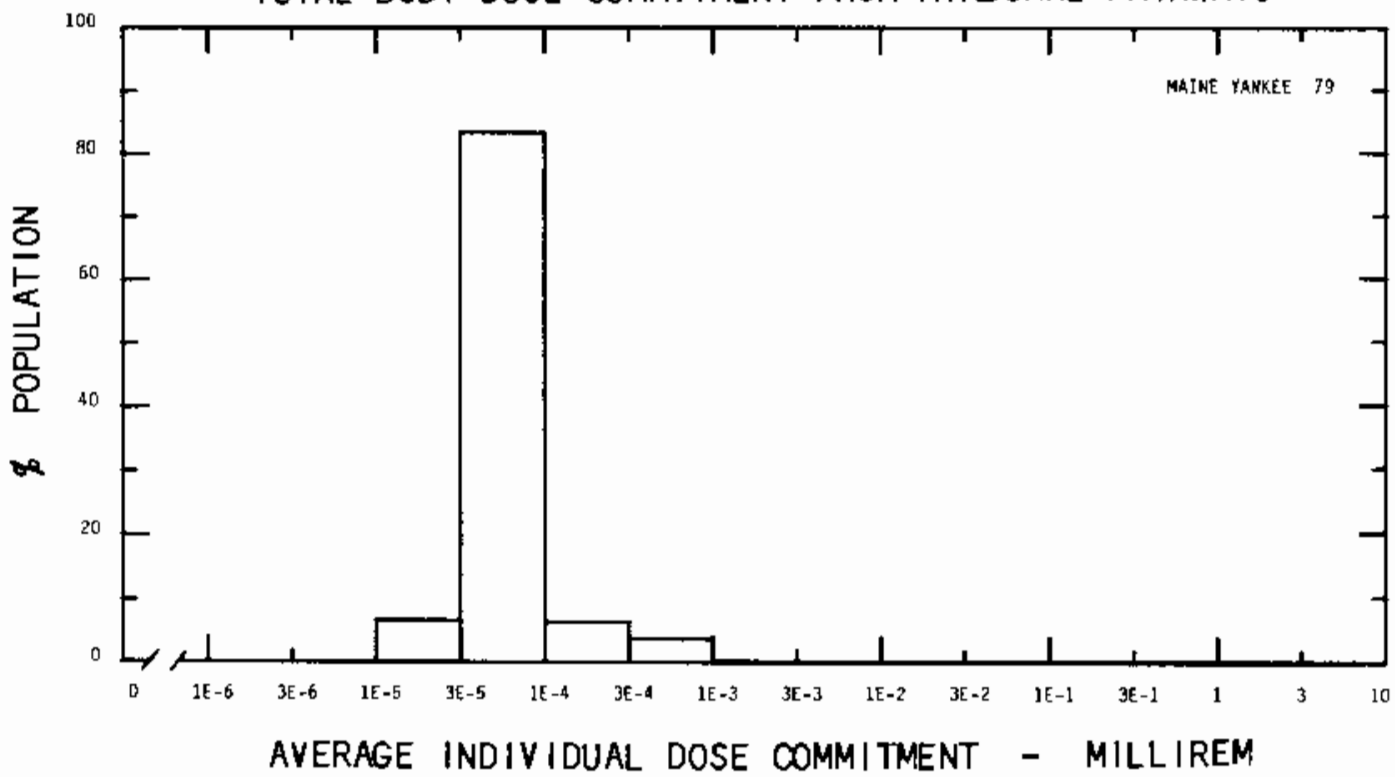


SIte: MILLSTONE POINT

Locatlon: N $41.3086^{\circ}$
WATERFOPO, CONNECTICUT

พ $72.1675^{\circ}$

POPULATION DATA

Total Population Within 20-to-80-km Region: 2.5E6

Major Metropolitan Centers within Region:

Center

Hartford SISA

New Haven SMSA

Narvick

Waterbury
Population

840,000

770,000

86,000

110,000
Location

$72 \mathrm{~km}$

$64 \mathrm{~km}$

$80 \mathrm{~km}$ ME

$70 \mathrm{~km} \mathrm{WN}$

SITE SPECIFIC DATA - AIRBORNE PATHWAYS

Average Annual State Production

of Crops and Anlmal Products

In 80-km Radius Circle

Regional Productivity Factor:

AnImal Grazing Factor:

Meteorology Perlod of Record: 1 JAN 74 - 31 DEC 74 Recovery: 95\%
Veg: 3.2E7 kilogram

MIlk: 4.4E8 liter

Meat: 2.0E7 kllogram

0.6

0.6

SITE SPECIFIC DATA - WATERBORNE PATHWAYS VIa NIANTIC BAY

FIsh:

Invertebrates:
Average DIlution Flow

from Plant: $710 \mathrm{ft}^{3} / \mathrm{s}$

Edible Harvest: $9.1 \mathrm{E} 4 \mathrm{~kg} / \mathrm{yr}$

Dilution Factor: $\mathbf{0 . 0 0 1}$

Edible Harvest: $9.1 \mathrm{E4} \mathrm{kg} / \mathrm{yr}$

Dilution Factor: $\mathbf{0 . 0 0 2}$ 
POPULATION DOSE-COMMITMENT ESTIMATES AND

AVERAGE INDIVIDUAL DOSE-CONMITMENT HISTOGRAM FOR

\section{MILLSTONE POINT 1 NND 2}

Dose Commitments (person-rem) from Liquid Pathways

Iotal Body GL-LLI Thyrold Bone Liver

$\begin{array}{llllll}\text { Infant } & 0.0 \mathrm{E}+00 & 0.0 \mathrm{E}+00 & 0.0 \mathrm{E}+00 & 0.0 \mathrm{E}+00 & 0.0 \mathrm{E}+00 \\ \text { Child } & 7.7 \mathrm{E}-04 & 1.8 \mathrm{E}-03 & 3.8 \mathrm{E}-04 & 2.8 \mathrm{E}-04 & 6.2 \mathrm{E}-04 \\ \text { Teen } & 5.7 \mathrm{E}-04 & 3.7 \mathrm{E}-03 & 2.6 \mathrm{E}-04 & 1.7 \mathrm{E}-04 & 5.4 \mathrm{E}-04 \\ \text { Adult } & 3.7 \mathrm{E}-03 & 3.3 \mathrm{E}-02 & 1.7 \mathrm{E}-03 & 9.8 \mathrm{E}-04 & 3.3 \mathrm{E}-03 \\ \text { TOTAL } & 5.1 \mathrm{E}-03 & 3.8 \mathrm{E}-02 & 2.4 \mathrm{E}-03 & 1.4 \mathrm{E}-03 & 4.4 \mathrm{E}-03\end{array}$

Dose Commitments (person-rem) from Alrborne Pathways

Total Body GI-LLI Thyrold Bone Liver Lung

$\begin{array}{lllllll}\text { Infant } & 2.6 \mathrm{E}-02 & 2.6 \mathrm{E}-02 & 6.0 \mathrm{E}-01 & 2.7 \mathrm{E}-02 & 2.8 \mathrm{E}-02 & 2.8 \mathrm{E}-02 \\ \text { ChIId } & 2.9 \mathrm{E}-01 & 2.9 \mathrm{E}-01 & 3.3 \mathrm{E}+00 & 2.9 \mathrm{E}-01 & 3.0 \mathrm{E}-01 & 3.1 \mathrm{E}-01 \\ \text { Teen } & 2.1 \mathrm{E}-01 & 2.1 \mathrm{E}-01 & 1.4 \mathrm{E}+00 & 2.1 \mathrm{E}-01 & 2.1 \mathrm{E}-01 & 2.4 \mathrm{E}-01 \\ \text { Adult } & 1.3 \mathrm{E}+00 & 1.3 \mathrm{E}+00 & 5.2 \mathrm{E}+00 & 1.2 \mathrm{E}+00 & 1.3 \mathrm{E}+00 & 1.4 \mathrm{E}+00 \\ \text { TOTAL } & 1.8 \mathrm{E}+00 & 1.8 \mathrm{E}+00 & 1.1 \mathrm{E}+01 & 1.8 \mathrm{E}+00 & 1.8 \mathrm{E}+00 & 1.9 \mathrm{E}+00\end{array}$

Production/Consumption factors:

Produce: <1 Milk: <1 Meat: <1

FRACTION OF POPULATION RECEIVING AN INDICATED AVERAGE

TOTAL-BODY DOSE COMMITMENT FROM AIRBORNE PATHWAYS

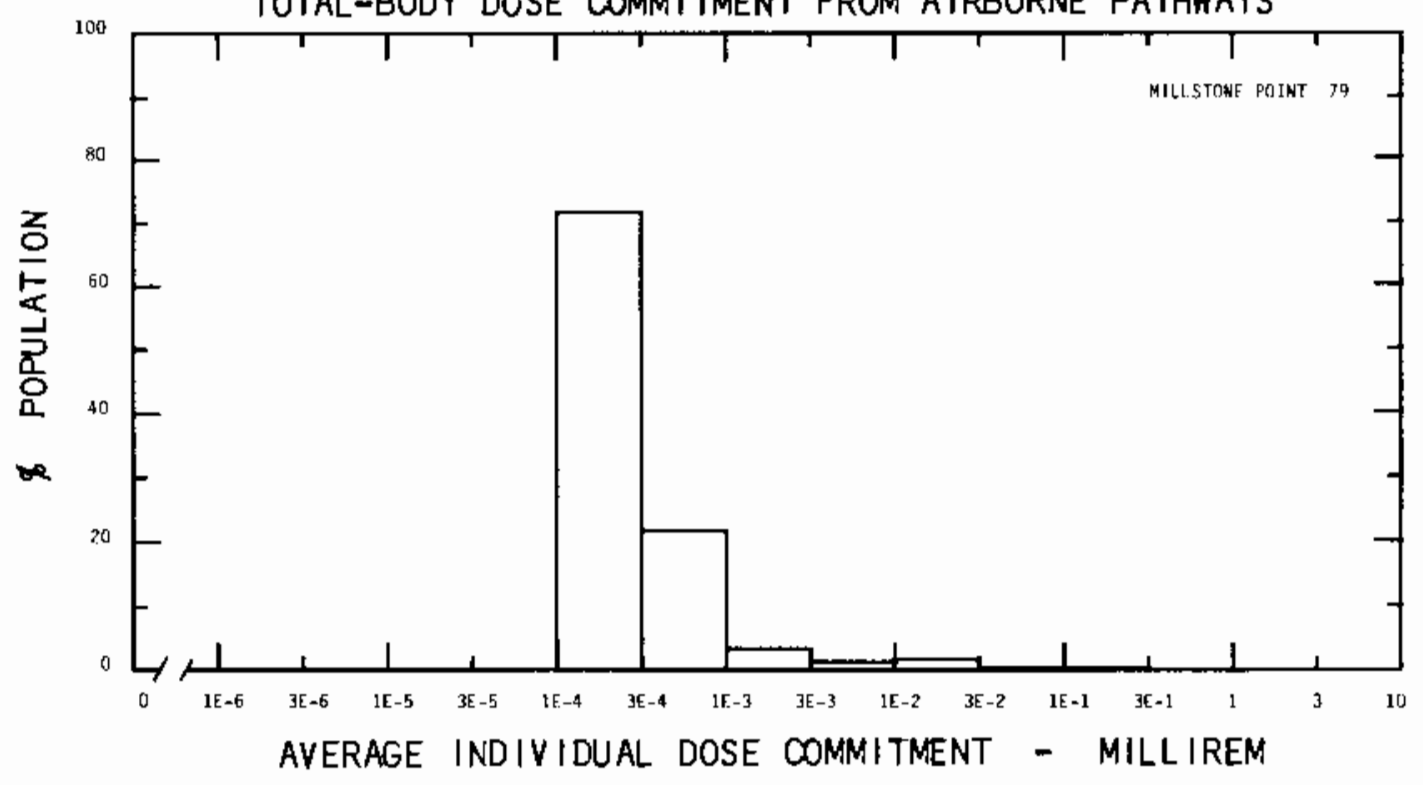


SIte: MONTICELLO

Lecation: N $45.3339^{\circ}$
MONTICELLO, MINESOTA

W $93.8486^{\circ}$

POPULATION DATA

Total Population within 20-to-80-km Reglon: 2.1E6

Major Metropolitan Centers within Reglon:

Center

MInneapolis-St. Paul SISA St. Cloud Anoka
Population

$2,100,000$ 42,000

14,000
Location

$64 \mathrm{~km} \quad \mathrm{SE}$

$37 \mathrm{~km} \mathrm{~N}$

$35 \mathrm{~km} \quad$ ESE

SITE SPECIFIC DATA - AIRBORNE PATHWAYS

Average Annual State Production

of Crops and AnImal Products

In 80-km Radius Circle

Regional Productivity Factor:

Animal Grazing Factor:

Meteorology Perlod of Record: 1 JAN 74 - 31 DEC 74 Recovery: 92\%

SITE SPECIFIC DATA - WATERBORNE PATHWAYS VIA MISSISSIPPI RIVER
Average River Flow
at Site: $4,600 \mathrm{ft}^{3 / \mathrm{s}}$
Orinking Water:
Exposed Population: (a)
Fish:
Edible Harvest: (a) $\mathrm{kg} / \mathrm{yr}$ (a) No radionucl ides released in Ilquid effluents reported (Tichler,
et al., 1981). 


\title{
POPULATION DOSE-COMMITMENT ESTIMATES AND \\ AVERAGE INDIVIDUAL DOSE-COMMITMENT HISTOGRAM FOR \\ MONTICELLO
}

\author{
Dose Commltments (person-rem) from Liquid Pathways \\ Iotal Body Gl-LLl Thyrodd Bone Liver
}

No Liquid Pathway Doses

Dose Commitments (person-rem) from Alrborne Pathways

Iotal Body Gl-LLl Thyrold Bone Llver Lung

$\begin{array}{lllllll}\text { Infant } & 2.4 \mathrm{E}-03 & 2.4 \mathrm{E}-03 & 1.8 \mathrm{E}-02 & 1.2 \mathrm{E}-03 & 2.5 \mathrm{E}-03 & 2.4 \mathrm{E}-03 \\ \text { Child } & 3.3 \mathrm{E}-02 & 3.2 \mathrm{E}-02 & 1.4 \mathrm{E}-01 & 1.9 \mathrm{E}-02 & 3.3 \mathrm{E}-02 & 3.3 \mathrm{E}-02 \\ \text { Teen } & 2.1 \mathrm{E}-02 & 2.1 \mathrm{E}-02 & 6.1 \mathrm{E}-02 & 1.1 \mathrm{E}-02 & 2.1 \mathrm{E}-02 & 2.2 \mathrm{E}-02 \\ \text { Adult } & 1.2 \mathrm{E}-01 & 1.2 \mathrm{E}-01 & 2.5 \mathrm{E}-01 & 6.1 \mathrm{E}-02 & 1.2 \mathrm{E}-01 & 1.2 \mathrm{E}-01 \\ \text { TOTAL } & 1.8 \mathrm{E}-01 & 1.7 \mathrm{E}-01 & 4.7 \mathrm{E}-01 & 9.3 \mathrm{E}-02 & 1.7 \mathrm{E}-01 & 1.8 \mathrm{E}-01\end{array}$

Production/Consumption factors:

Produce: <1 Milk: $1.4 \quad$ Meat: <1

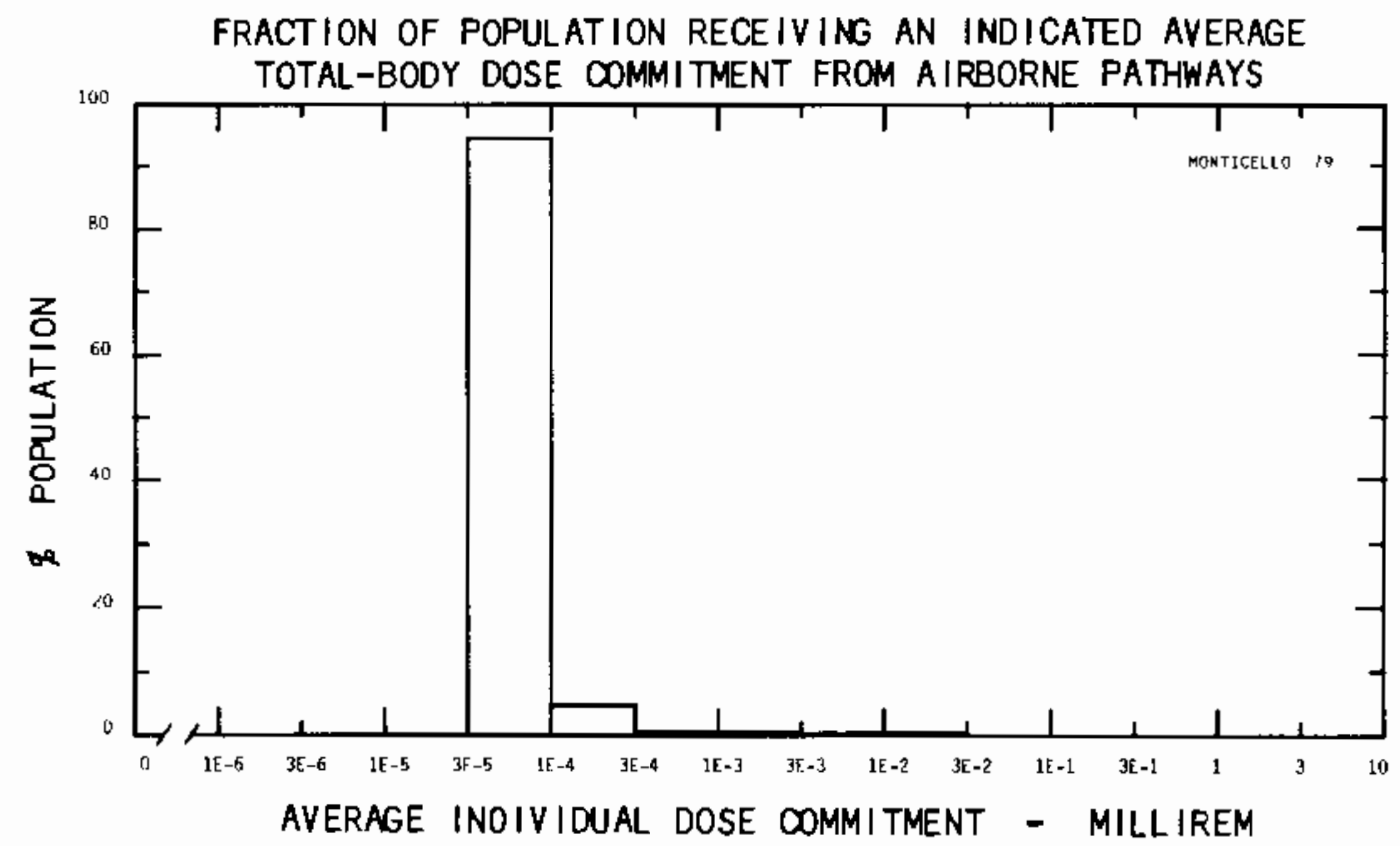


Site: NINE MILE POINT

Lecation:

N $43.5217^{\circ}$
OSWEGO, NEY YORK

W $76.3980^{\circ}$

POPULATION DATA

Total Population within 20-to-80-km Region: $\mathbf{8 . 1 E 5}$

Major Metropolitan Centers within Region:

Center

Syracuse SMSA

Auburn
Population

620,000

33,000
Location

$54 \mathrm{~km}$ SSE

$66 \mathrm{~km}$ SSW

SITE SPECIFIC DATA - AIRBORNE PATHWAYS

Average Annual State Production

of Crops and Animal Products

In 80-km Radius Circle

Regional Productivity Factor:

AnImal Grazing Factor:

Meteorology Perlod of Record: 1 JAN 74 - 31 DEC 75 Recovery: 97\%
Veg: 7.6E7 kilogram

MIIk: 7.0E8 IIter

Meat: 3.3E7 kilogram

0.7

0.5

SITE SPECIFIC DATA - WATERBORNE PATHWAYS vIa LAKE ONTARIO
Average DIlution,Flow
from Plant: $6 \mathrm{ft}^{3} / \mathrm{s}$
Drinking Water:
Exposed Population: 530,000
Dilution Factor: 0.01998
FIsh:
Edible Harvest: 7.3 E5 kofyr
Dilution Factor: $3.3 \mathrm{E}-3$ (b)

(a) Drinking water dilution factor estimated by averaging dllution factor derived from FES (1974) sultably weighted for population.

(b) Generic lake dilution factor of 0.01 reduced $1 / 3$ by diffuser (FES, 1974). 


\section{POPULATION DOSE-COMMITMENT ESTIMATES AND \\ AVERAGE INDIVIDUAL DOSE-COMMITMENT HISTOGRAM FOR \\ NINE MILE POINT}

Dose Commitments (person-rem) from LlquId Pathways

Iotal Body

GI-LLل Ihyrold

Bene

Lyer

Infant

Child

Teen

Adult

TOTAL

Infant

Child

Teen

Adult

TOTAL
2. $4 \mathrm{E}-01$

8. $2 \mathrm{E}+00$

$1.1 \mathrm{E}+01$

1. $2 \mathrm{E}+02$

$1.4 \mathrm{E}+02$
4. $4 \mathrm{E}-02$

9.6E-01

1. $0 E+00$

9. $0 \mathrm{E}+00$

$1.1 \mathrm{E}+01$
5.1E-03

5.7E-02

$2.2 \mathrm{E}-02$

1.9E-01

2.7E-01
1. $8 \mathrm{E}+00$

4. $2 E+01$

1. $9 \mathrm{E}+01$

$1.1 E+02$

$1.7 \mathrm{E}+02$
2. $1 \mathrm{E}+00$

4. $4 E+01$

$2.7 \mathrm{E}+01$

1. $7 \mathrm{E}+02$

$2.4 \mathrm{E}+02$

Dose Commitments (person-rem) from Alrborne Pathways

\section{Iotal Body GJ-LLل Thyroid Bone Llver Lung}

$\begin{array}{llllll}1.2 \mathrm{E}-03 & 1.1 \mathrm{E}-03 & 1.6 \mathrm{E}-02 & 1.3 \mathrm{E}-03 & 1.4 \mathrm{E}-03 & 1.2 \mathrm{E}-03 \\ 1.4 \mathrm{E}-02 & 1.3 \mathrm{E}-02 & 1.2 \mathrm{E}-01 & 1.7 \mathrm{E}-02 & 1.5 \mathrm{E}-02 & 1.4 \mathrm{E}-02 \\ 9.7 \mathrm{E}-03 & 9.6 \mathrm{E}-03 & 4.7 \mathrm{E}-02 & 1.0 \mathrm{E}-02 & 1.0 \mathrm{E}-02 & 1.0 \mathrm{E}-02 \\ 5.7 \mathrm{E}-02 & 5.7 \mathrm{E}-02 & 1.8 \mathrm{E}-01 & 5.6 \mathrm{E}-02 & 5.8 \mathrm{E}-02 & 5.8 \mathrm{E}-02 \\ 8.2 \mathrm{E}-02 & 8.1 \mathrm{E}-02 & 3.6 \mathrm{E}-01 & 8.4 \mathrm{E}-02 & 8.4 \mathrm{E}-02 & 8.4 \mathrm{E}-02\end{array}$

Production/Consumption factors:

Produce: $<1$

MIIK: $\quad 4.6$

Meat: $<1$

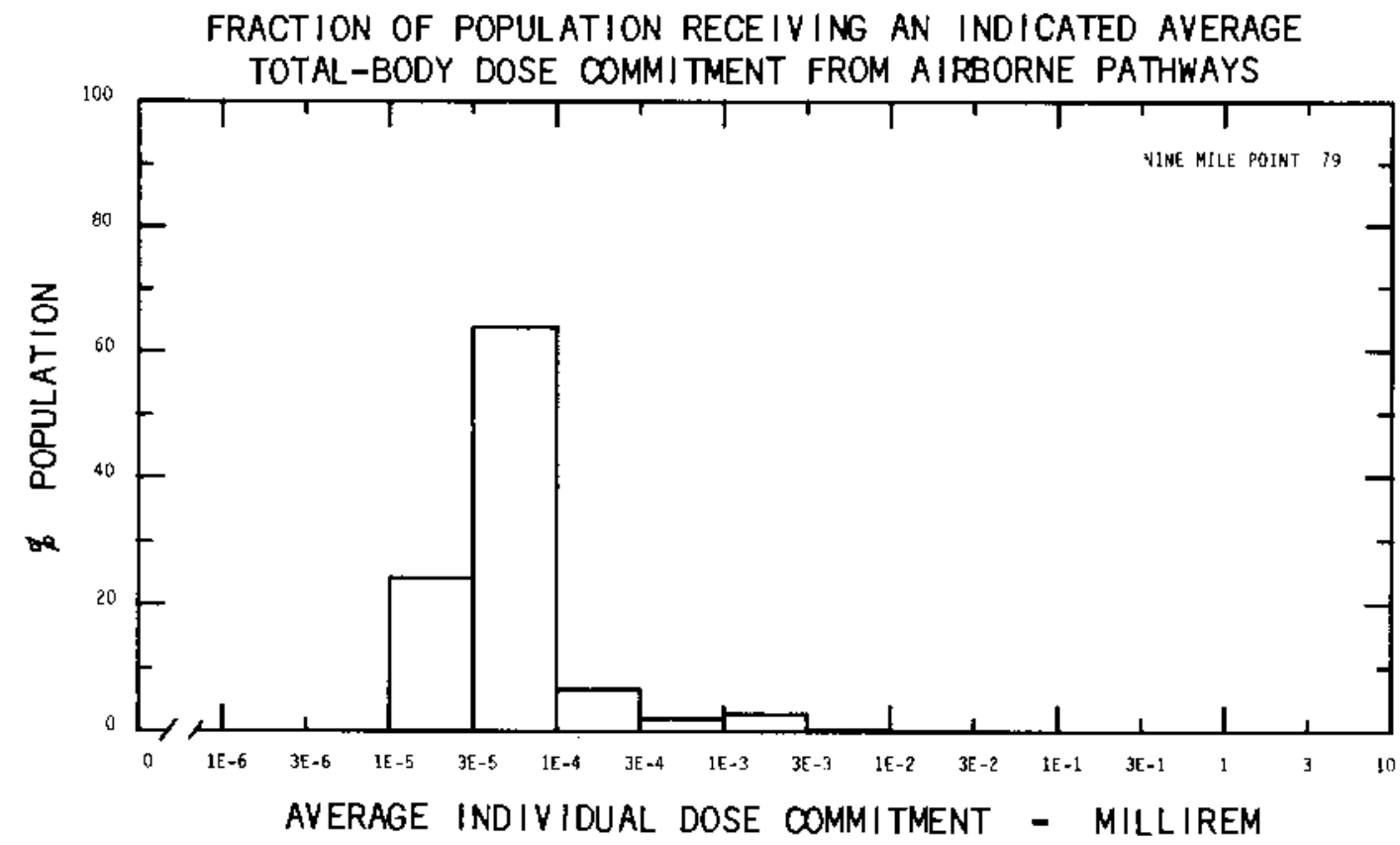




\section{SIte: MORTH ANNA}

Location: N $38.0631^{\circ}$
LOUISA COUNTY, VIRGINIA

W $77.7867^{\circ}$

POPULATION DATA

Total Population within 20-to-80-km Reglon: 9.6E5

Major Metropolitan Centers within Reglon:

Center:

Char lottesville

RIcheond

Fredricksburg
Population

$\begin{array}{rll}43,000 & 64 \mathrm{~km} & W \\ 280,000 & 48 \mathrm{~km} & \text { SE } \\ 16,000 & 40 \mathrm{~km} & \text { NE }\end{array}$

SITE SPECIFIC DATA - AIRBORNE PATHWAYS

Average Annual State Production

Of Crops and Animal Products

In 80-km Radius Circle

Reglonal Productivity Factor:

Animal Grazing Factor:

Meteorology Perlod of Record: 1 APR 74 - 31 APR 75 Recovery: 99\%
Veg: $3.5 \mathrm{E} 7$ kilogram

Milk: 1.5EB I iter

Meat: 7.4E7 kilogram

0.9

0.7

SITE SPECIFIC DATA - WATERBORNE PATHWAYS VIa LAKE ANNA (a)

$\begin{array}{ll} & \text { Average Dilution Flow from } \\ & \text { Plant: } 1,700 \mathrm{ft} / \mathrm{s} \\ \text { Drinking Water: } & \text { Exposed Population: None } \\ \text { Fish: } & \text { Edible Harvest: } 7.3^{(\mathrm{b})} \mathrm{kg} / \mathrm{yr} \\ & \text { Dllution Factor: } 0.001\end{array}$

(a) Reconcentration of radionuclides in lake accounted for (FES, 1973).

(b) Average individual consumption rates as given in the FES (1973) were used in I leu of catch data. 


\section{POPULATION DOSE-COMMITMENT ESTIMATES AND AVERAGE INDIVIDUAL DOSE-COMMITMENT HISTOGRAM FOR \\ MORTH ANW}

Dose Commltments (person-rem) from Llquid Pathways

Iotal Body GL-LLl Thyrold Bone Llyer

Infant

$\begin{array}{lllll}0.0 \mathrm{E}+00 & 0.0 \mathrm{E}+00 & 0.0 \mathrm{E}+00 & 0.0 \mathrm{E}+00 & 0.0 \mathrm{E}+00 \\ 2.0 \mathrm{E}-01 & 7.7 \mathrm{E}-03 & 4.5 \mathrm{E}-03 & 9.8 \mathrm{E}-01 & 1.1 \mathrm{E}+00 \\ 3.9 \mathrm{E}-01 & 1.5 \mathrm{E}-02 & 3.3 \mathrm{E}-03 & 5.9 \mathrm{E}-01 & 9.6 \mathrm{E}-01 \\ 4.2 \mathrm{E}+00 & 1.2 \mathrm{E}-01 & 2.2 \mathrm{E}-02 & 3.4 \mathrm{E}+00 & 5.7 \mathrm{E}+00 \\ 4.8 \mathrm{E}+00 & 1.4 \mathrm{E}-01 & 3.0 \mathrm{E}-02 & 5.0 \mathrm{E}+00 & 7.9 \mathrm{E}+00\end{array}$

Dose Commitments (person-rem) from Airborne Pathways

Iotal Body Gl-LL Ihyrold Bone Llyer Lung

$\begin{array}{lllllll}\text { Infant } & 8.1 \mathrm{E}-04 & 7.7 \mathrm{E}-04 & 2.9 \mathrm{E}-02 & 9.5 \mathrm{E}-04 & 9.9 \mathrm{E}-04 & 8.5 \mathrm{E}-04 \\ \text { ChIld } & 8.9 \mathrm{E}-03 & 8.6 \mathrm{E}-03 & 1.6 \mathrm{E}-01 & 9.9 \mathrm{E}-03 & 1.0 \mathrm{E}-02 & 9.6 \mathrm{E}-03 \\ \text { Teen } & 6.4 \mathrm{E}-03 & 6.3 \mathrm{E}-03 & 6.5 \mathrm{E}-02 & 6.6 \mathrm{E}-03 & 6.8 \mathrm{E}-03 & 7.5 \mathrm{E}-03 \\ \text { Adult } & 3.9 \mathrm{E}-02 & 3.8 \mathrm{E}-02 & 2.2 \mathrm{E}-01 & 3.9 \mathrm{E}-02 & 3.9 \mathrm{E}-02 & 4.2 \mathrm{E}-02 \\ \text { TOTAL } & 5.5 \mathrm{E}-02 & 5.4 \mathrm{E}-02 & 4.8 \mathrm{E}-01 & 5.6 \mathrm{E}-02 & 5.7 \mathrm{E}-02 & 6.0 \mathrm{E}-02\end{array}$

Production/Consumption factors:

Produce: $<1 \quad$ Milk: $1.1 \quad$ Meat: <1

FRACTION OF POPULATION RECEIVING AN INDICATED AVERAGE TOTAL-BODY DOSE COMMITMENT FROM AIRBORNE PATHWAYS

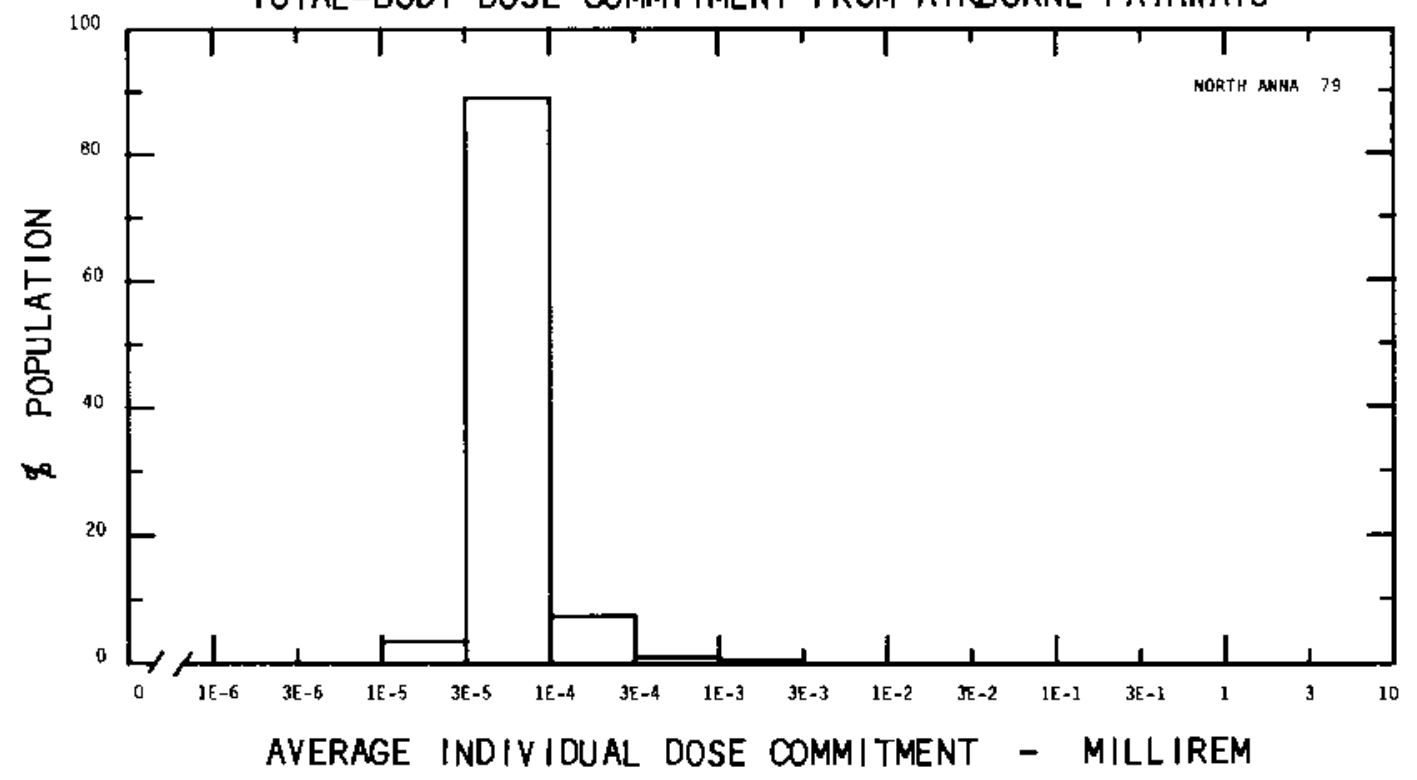


Site: OCONEE

Lecation:

N $34.7500^{\circ}$

OCONEE COUNTY, SOUTH CAROL INA

W $83.0458^{\circ}$

POPULATION DATA

Total Population with in 20-to-80-km Reglon: 7.5E5

Major Metropolitan Centers Within Region:

Center

Population

Lecation

Anderson

31,000

$39 \mathrm{~km} \quad \mathrm{SE}$

Greenvill le

69,000

$47 \mathrm{~kg} \quad \mathrm{E}$

SITE SPECIFIC DATA - AIRBORNE PATHWAYS

Average Annual State Production

of Crops and Animal Products

In 80-km Radius Circle

Reglonal Productivity Factor:

Animal Grazlng Factor:

Meteorology Perlod of Record: 1 JAN 75 - 31 DEC 75 Recovery: 86\%
Veg: 7.4E6 kilogram

Milk: 5.7E7 liter

Meat: $5.0 \mathrm{E7}$ kllogram

1

0.7

SITE SPECIFIC DATA - WATERBORNE PATHWAYS VIa HARTWELL RES, On KEOWEE RIVER Average River Flow
at Site: $1,100 \mathrm{ft} / \mathrm{s}$

Drinking Water:

Exposed Population: 45,000 Dilution Factor: 1

Fish:

Edible Harvest: (a) $\mathrm{kg} / \mathrm{yr}$ Dilution Factor: $0.01^{(b)}$

(a) No fish catch data given in FES, so generic consumption rates used (Table $A-1$ ).

(b) Ten percent of population obtain $10 \%$ of their fish diet from Hartwell Reservolr (FES, 1972). 


\section{POPULATION DOSE-COMMITMENT ESTIMATES AND AVERAGE INDIVIDUAL DOSE-COMMITMENT HISTOGRAM FOR \\ COONEE 1, 2 AND 3}

Dose Commitments (person-rem) from Liquid Pathways Iotal Body Gl-LL Ihyrold Bone Liver

$\begin{array}{llllll}\text { Infant } & 3.7 \mathrm{E}-02 & 3.1 \mathrm{E}-02 & 2.3 \mathrm{E}-01 & 5.6 \mathrm{E}-02 & 5.6 \mathrm{E}-02 \\ \text { Child } & 5.6 \mathrm{E}-01 & 3.7 \mathrm{E}-01 & 1.8 \mathrm{E}+00 & 1.2 \mathrm{E}+00 & 1.2 \mathrm{E}+00 \\ \text { Teen } & 4.0 \mathrm{E}-01 & 1.7 \mathrm{E}-01 & 5.7 \mathrm{E}-01 & 5.3 \mathrm{E}-01 & 7.5 \mathrm{E}-01 \\ \text { Adult } & 4.0 \mathrm{E}+00 & 1.4 \mathrm{E}+00 & 4.2 \mathrm{E}+00 & 3.3 \mathrm{E}+00 & 4.8 \mathrm{E}+00 \\ \text { TOTAL } & 5.0 \mathrm{E}+00 & 2.0 \mathrm{E}+00 & 6.7 \mathrm{E}+00 & 5.1 \mathrm{E}+00 & 6.9 \mathrm{E}+00\end{array}$

Dose Commitments (person-rem) from Alrborne Pathways Iotal Body Gl-LL Ihyrold Bone Liver Lung

$\begin{array}{lllllll}\text { Infant } & 1.1 \mathrm{E}-02 & 1.1 \mathrm{E}-02 & 5.1 \mathrm{E}-02 & 1.1 \mathrm{E}-02 & 1.1 \mathrm{E}-02 & 1.2 \mathrm{E}-02 \\ \text { Child } & 1.3 \mathrm{E}-01 & 1.3 \mathrm{E}-01 & 3.6 \mathrm{E}-01 & 1.3 \mathrm{E}-01 & 1.3 \mathrm{E}-01 & 1.4 \mathrm{E}-01 \\ \text { Teen } & 9.3 \mathrm{E}-02 & 9.3 \mathrm{E}-02 & 1.9 \mathrm{E}-01 & 9.2 \mathrm{E}-02 & 9.3 \mathrm{E}-02 & 1.1 \mathrm{E}-01 \\ \text { Adult } & 5.6 \mathrm{E}-01 & 5.7 \mathrm{E}-01 & 9.3 \mathrm{E}-01 & 5.6 \mathrm{E}-01 & 5.6 \mathrm{E}-01 & 6.2 \mathrm{E}-01 \\ \text { TOTAL } & 7.9 \mathrm{E}-01 & 8.0 \mathrm{E}-01 & 1.5 \mathrm{E}+00 & 7.9 \mathrm{E}-01 & 7.9 \mathrm{E}-01 & 8.9 \mathrm{E}-01\end{array}$

Production/Consumption factors:

Produce: $<1 \quad$ Milk: $<1 \quad$ Meat: $<1$

FRACTION OF POPULATION RECEIVING AN INDICATED AVERAGE

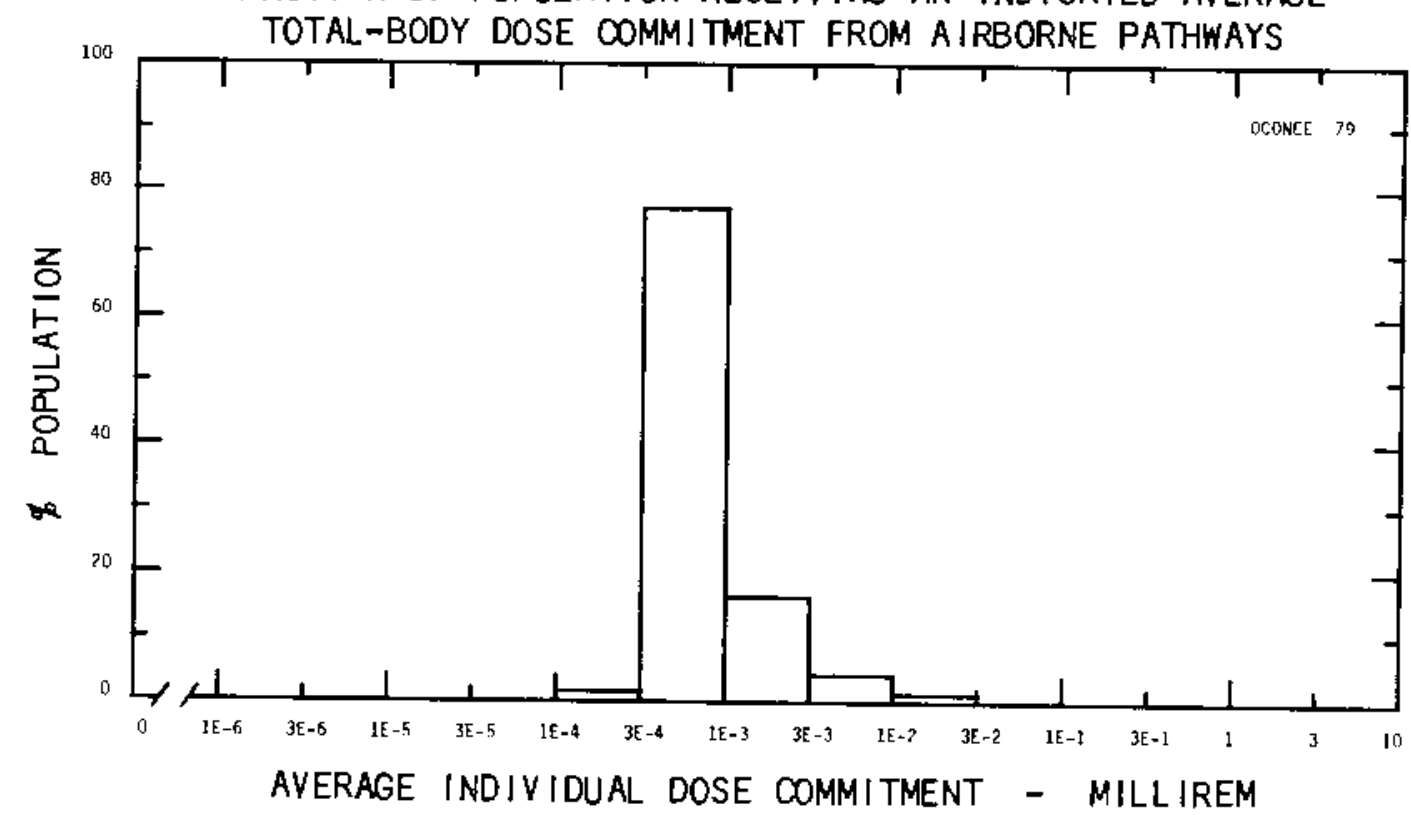


SIte: OYSTER CREEK

OYSTER CREEK. NEY JERSEY

Lecation: N $39.8139^{\circ}$ W $74.2114^{\circ}$

POPULATION DATA

Total Population within 20-to-80-km Region: 3.3 E6

Major Metropolitan Centers Within Region:

Center

Atlantic City

Trenton
Population

49,000

110,000
Lecation

$56 \mathrm{~km} \quad \mathrm{SSW}$

$64 \mathrm{~km} \mathrm{NM}$

SITE SPECIFIC DATA - AIRBORNE PATHWAYS

Average Annual State Production

of Crops and Animal Products

In 80-km Radius Circle

Regional Productivity Factor:

AnImal Grazlng Factor:

Meteorology Perlod of Record:
Veg: $7.4 \mathrm{E} 7$ kilogram

Milk: 2.7E8 liter

Meat: $2.4 \mathrm{E} 7 \mathrm{kilogram}$

0.5

0.6

15 FEB $66-31$ DEC 68 Recovery: $63 \%$

$\begin{array}{ll} & \begin{array}{l}\text { Average Dilution Flow } \\ \text { from Plant: } 2,000 \mathrm{ft}^{3} / \mathrm{s}\end{array} \\ \text { Fish: } & \text { Edible Harvest: } 2.1(\mathrm{a}) \mathrm{kg} / \mathrm{yr} \\ & \text { Dilution Factor: } 0.01(\mathrm{~b}) \\ & \text { Edible Harvest: } 0.96(\mathrm{a}) \mathrm{kg} / \mathrm{yr} \\ \text { Invertebrates: } & \text { Dilution Factor: } 0.01(\mathrm{~b})\end{array}$

(a) Average Individual consumption rate as given in the FES (1974) used in I leu of catch data.

(b) $10 \%$ of seafood eaten assumed caught in bay waters diluted to $10 \%$ of that of discharge canal (FES, 1974). 


\section{POPULATION DOSE-COMMITMENT ESTIMATES AND AVERAGE INDIVIDUAL DOSE-COMMITMENT HISTOGRAM FOR OYSTER CREEK}

Dose Com Itments (person-rem) from LIquid Pathways

Iotal Body

GL-LLI

Ihyrold

Bone

Liver

Infant

$0.0 \mathrm{E}+00$

$0.0 \mathrm{E}+00$

$0.0 \mathrm{E}+00$

$0.0 \mathrm{E}+00$

$0.0 E+00$

Child

1.2E-05

2.3E-05

3. $2 \mathrm{E}-05$

3.0E-05

3.1E-05

Teen

$1.3 \mathrm{E}-05$

4.5E-05

2.3E-05

1. $8 \mathrm{E}-05$

2.6E-05

Adult

1. $2 \mathrm{E}-04$

3. $8 \mathrm{E}-04$

1. $5 \mathrm{E}-04$

1. $0 \mathrm{E}-04$

$1.5 \mathrm{E}-04$

TOTAL

1. $4 \mathrm{E}-04$

4.5E-04

2.1E-04

$1.5 \mathrm{E}-04$

2.1E-04

Dose Commitments (person-rem) from Alrborne Pathways

\section{Iotal Body Gl-LLl Ihyrold Bone Liver Lung}

$\begin{array}{lllllll}\text { Infant } & 3.2 \mathrm{E}+00 & 3.2 \mathrm{E}+00 & 7.7 \mathrm{E}+00 & 3.2 \mathrm{E}+00 & 3.2 \mathrm{E}+00 & 3.2 \mathrm{E}+00 \\ \text { ChIld } & 3.5 \mathrm{E}+01 & 3.5 \mathrm{E}+01 & 6.7 \mathrm{E}+01 & 3.6 \mathrm{E}+01 & 3.5 \mathrm{E}+01 & 3.6 \mathrm{E}+01 \\ \text { Teen } & 2.6 \mathrm{E}+01 & 2.6 \mathrm{E}+01 & 4.0 \mathrm{E}+01 & 2.6 \mathrm{E}+01 & 2.6 \mathrm{E}+01 & 2.7 \mathrm{E}+01 \\ \text { Aduit } & 1.6 \mathrm{E}+02 & 1.6 \mathrm{E}+02 & 2.1 \mathrm{E}+02 & 1.6 \mathrm{E}+02 & 1.6 \mathrm{E}+02 & 1.6 \mathrm{E}+02 \\ \text { TOTAL } & 2.2 \mathrm{E}+02 & 2.2 \mathrm{E}+02 & 3.3 \mathrm{E}+02 & 2.2 \mathrm{E}+02 & 2.2 \mathrm{E}+02 & 2.3 \mathrm{E}+02\end{array}$

Production/Consumption factors:

Produce: $<1 \quad$ Milk: <1 Meat: <1

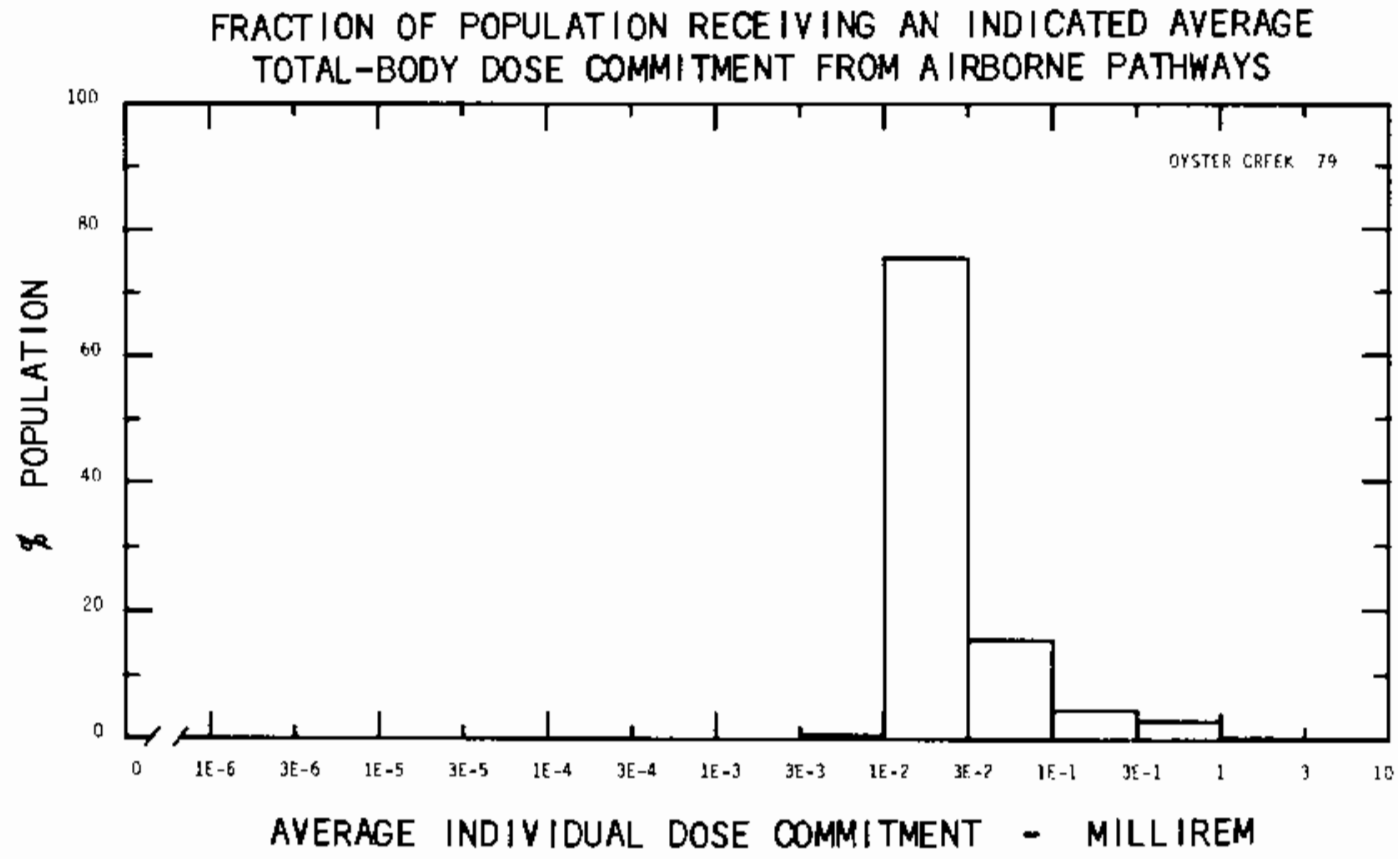


SIte: PALISADES

Location:

$\mathrm{N} \quad 42.3233^{\circ}$

POPULATION DATA

Total Population Within 20-to-80-km Region: 1.0E6

Major Metropolitan Centers WithIn Region:

Center.

Kal amazoo

South Bend SMSA

Michigan City
Population

89,000

290,000

41,000
Location

$56 \mathrm{~km} \quad \mathrm{E}$

$72 \mathrm{~km} S$

$66 \mathrm{~km} \quad \mathrm{SW}$

SITE SPECIFIC DATA - AIRBORNE PATHWAYS

Average Annual State Production

of Crops and AnImal Products

In 80-km Radlus Circle

Regional Productivity Factor:

AnImal Grazing Factor:

Meteorology Perlod of Record: 1 SEP 73 - 31 AUG 74 Recovery: 67\%
Veg: 6.8E7 kilogram

Milk: 2.9E8 Ilter

Meat: 4.5E7 kllogram

0.6

0.5

SITE SPECIFIC DATA - WATERBORNE PATHWAYS VIa LAKE MICHIGAN

Average Dilution Flow from

Plant: $98 \mathrm{ft}^{3} / \mathrm{s}$

Drinking Water:

Exposed Population: 48,000

Dilution Factor: $3.5 \mathrm{E}-3$ (a)

Fish:

Edible Harvest: (b) $\mathrm{kg} /(65)$
Dilution Factor: 0.001

(a) Drinking water dilution factor estimated by averaging dilution factor derived from FES (1972) sultably weighted for population.

(b) Since the average individual consumption rate of $20 \mathrm{~g} / \mathrm{d}$ as assumed in FES (1972) seemed unreasonably large, generic rates were used (Table A-1). 
POPULATION DOSE-COMMITMENT ESTIMATES AND

AVERAGE INDIVIDUAL DOSE-COMMITMENT HISTOGRAM FOR

\section{PN ISADES}

Dose Commitments (person-rem) from Liquid Pathways

TotalBody GL-LLI Thyrold Bone Llyer

$\begin{array}{llllll}\text { Infant } & 1.9 \mathrm{E}-04 & 1.9 \mathrm{E}-04 & 2.1 \mathrm{E}-04 & 2.3 \mathrm{E}-05 & 2.2 \mathrm{E}-04 \\ \text { Child } & 6.8 \mathrm{E}-03 & 5.4 \mathrm{E}-03 & 2.4 \mathrm{E}-03 & 2.6 \mathrm{E}-02 & 3.0 \mathrm{E}-02 \\ \text { Teen } & 9.5 \mathrm{E}-03 & 7.5 \mathrm{E}-03 & 9.8 \mathrm{E}-04 & 1.5 \mathrm{E}-02 & 2.4 \mathrm{E}-02 \\ \text { Adult } & 1.0 \mathrm{E}-01 & 6.5 \mathrm{E}-02 & 8.1 \mathrm{E}-03 & 8.7 \mathrm{E}-02 & 1.4 \mathrm{E}-01 \\ \text { TOTAL } & 1.2 \mathrm{E}-01 & 7.8 \mathrm{E}-02 & 1.2 \mathrm{E}-02 & 1.3 \mathrm{E}-01 & 2.0 \mathrm{E}-01\end{array}$

Dose Commitments (person-rem) from Airborne Pathways IotalBody Shl-Lلl Ihyrold Bone Liver Lung

$\begin{array}{lllllll}\text { Infant } & 5.3 \mathrm{E}-05 & 4.2 \mathrm{E}-05 & 7.7 \mathrm{E}-03 & 6.6 \mathrm{E}-05 & 9.2 \mathrm{E}-05 & 5.6 \mathrm{E}-05 \\ \text { ChIld } & 6.2 \mathrm{E}-04 & 5.4 \mathrm{E}-04 & 4.7 \mathrm{E}-02 & 6.1 \mathrm{E}-04 & 8.5 \mathrm{E}-04 & 7.3 \mathrm{E}-04 \\ \text { Teen } & 4.1 \mathrm{E}-04 & 3.9 \mathrm{E}-04 & 1.8 \mathrm{E}-02 & 3.1 \mathrm{E}-04 & 4.9 \mathrm{E}-04 & 5.4 \mathrm{E}-04 \\ \text { Adult } & 2.2 \mathrm{E}-03 & 2.2 \mathrm{E}-03 & 6.2 \mathrm{E}-02 & 1.5 \mathrm{E}-03 & 2.4 \mathrm{E}-03 & 2.7 \mathrm{E}-03 \\ \text { TOTAL } & 3.3 \mathrm{E}-03 & 3.2 \mathrm{E}-03 & 1.3 \mathrm{E}-01 & 2.5 \mathrm{E}-03 & 3.8 \mathrm{E}-03 & 4.1 \mathrm{E}-03\end{array}$

Production/Consumption factors:

Produce: <1 Milk: 1.3 Meat: <1

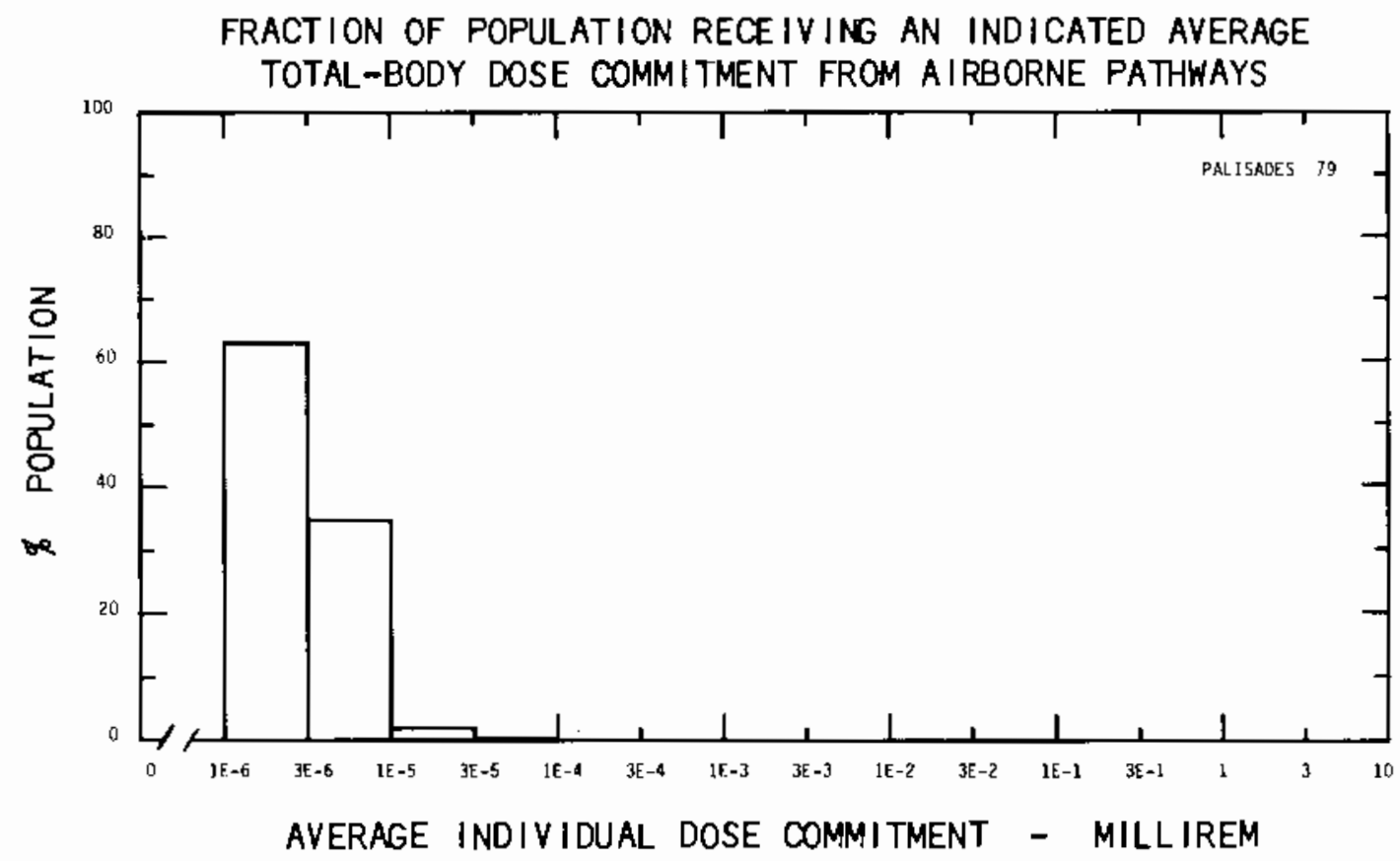


Site: PEACH BOTTOM

YORK COUNTY, PENMSYLYANIA

Lecation: N $39.7592^{\circ}$ W $76.2689^{\circ}$

POPULATION DATA

Total Population Within 20-to-80-km Region: 4.1E6

Major Metropolitan Centers within Reglon:

Center

Lancaster SMSA

Baltitnore SMSA

WIImIngton SMSA

Reading SUSA
Population

320,000

$2,100,000$

500,000

290,000
Location

$31 \mathrm{~km} \mathrm{~N}$

$58 \mathrm{~km}$ sit

$64 \mathrm{~km} \quad E$

$72 \mathrm{~km} \quad \mathrm{NNE}$

SITE SPECIFIC DATA - AIRBORNE PATHWAYS

Average Annual State Production

Of Crops and Anlmal Products

In 80-km Radius Circle

Reglonal Productivity Factor:

AnImal Grazing Factor:

Meteorology Perlod of Record:

NUG $67-31$ JUL 71

Veg: 5.3E7 kllogram

Milk: 5.3E8 liter

Meat: $5.4 E 7$ kilogram

0.95

0.6

SITE SPECIFIC DATA - WATERBORNE PATHWAYS VIA SUSQUEHANNA RIVER

Average River Flow

$$
\text { at Site: } 36,000 \mathrm{ft}^{3} / \mathrm{s}
$$

Drinking Water:

Exposed Population: 2.1E6

Dllution Factor: 1

Fish:
Edible Harvest: (a) $\mathrm{kq} / \mathrm{yr}^{r}$
Dilution Factor: 0.001

(a) No fish catch data given in FES (1974), thus generlc consumption rates used (Table A-1).

(b) One percent of people obtaln $10 \%$ of their fish diet from river downstream from plant (FES, 1973). 


\section{POPULATION DOSE-COMMITMENT ESTIMATES AND AVERAGE INDIVIDUAL DOSE-COMMITMENT HISTOGRAM FOR PENCH BOTTON 2 ND 3}

Dose Commitments (person-rem) from Liquid Pathways

Iotal Body

Gl-LLI Thyrold

Bone

Luer

Infant

$8.1 \mathrm{E}-02$

$1.5 \mathrm{E}-02$

2.1E+00

$5.4 \mathrm{E}-01$

$7.8 \mathrm{E}-01$

Chlld

$1.5 \mathrm{E}+00$

1. $5 \mathrm{E}-01$

1. $5 \mathrm{E}+01$

$5.8 \mathrm{E}+00$

$7.3 \mathrm{E}+00$

Teen

1. $2 \mathrm{E}+00$

9. $4 \mathrm{E}-02$

4. $4 \mathrm{E}+00$

$1.6 \mathrm{E}+00$

$2.8 \mathrm{E}+00$

Adult

1. $3 E+01$

8.1E-01

$3.1 \mathrm{E}+01$

9.7E+00

$1.8 \mathrm{E}+01$

TOTAL

$1.6 \mathrm{E}+01$

1. $1 \mathrm{E}+00$

$5.2 \mathrm{E}+01$

$1.8 \mathrm{E}+01$

$2.9 \mathrm{E}+01$

Dose Commitments (person-rem) from Alrborne Pathways

TotalBody Gl-LLl Thyrold Bone Llver Lung

Infant

Child

Teen

Adult

TOTAL
2.1E-01

$2.3 E+00$

$1.7 \mathrm{E}+00$

$1.0 \mathrm{E}+01$

$1.4 \mathrm{E}+01$

\subsection{E-01}

$2.3 E+00$

$1.7 \mathrm{E}+00$

$1.0 \mathrm{E}+01$

$1.4 \mathrm{E}+01$
$6.9 \mathrm{E}-01$

$5.1 E+00$

2. $9 \mathrm{E}+00$

1. $5 \mathrm{E}+01$

2.1E-01

$2.3 E+00$

$1.7 \mathrm{E}+00$

$1.0 \mathrm{E}+01$

$2.3 E+01$

$1.5 \mathrm{E}+01$

2.1E-01

$2.3 \mathrm{E}+00$

$1.7 \mathrm{E}+00$

2.2E-01

$2.5 \mathrm{E}+00$

$2.0 \mathrm{E}+00$

$1.0 E+01$

$1.1 E+01$

$1.4 E+01$

$1.6 \mathrm{E}+01$

Production/Consumption factors:

Produce: < 1

Milk: <1

Meat: <1

FRACTION OF POPULATION RECEIVING AN INDICATED AVERAGE

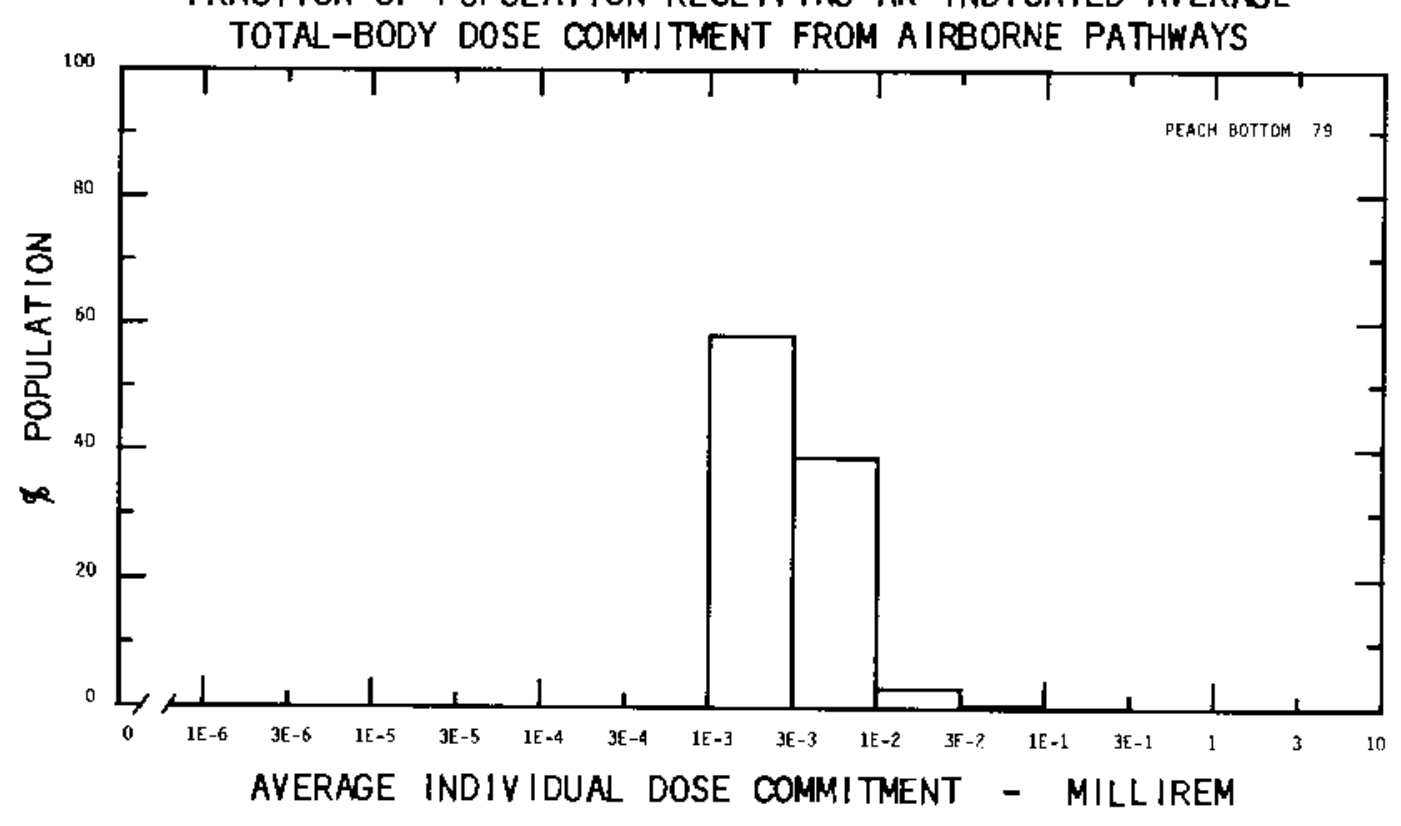


SIte: PILGRIM

Lecation:

W $70.5778^{\circ}$

POPULAT ION DATA

Total Population within 20-to-80-km Reglon: 4.3E6

Major Metropolitan Centers with in Region:

Center

Boston SISA

Providence SMSA

Brockton

New Bedford
Population

$3,400,000$

780,000

90,000

100,000
Lecation

$64 \mathrm{~km} \mathrm{~m}$

$70 \mathrm{~km}$ WSW

$40 \mathrm{~km}$ WN

$45 \mathrm{~km} \quad \mathrm{SH}$

SITE SPECIFIC DATA - AIRBORNE PATHWAYS

Average Annual State Production

of Crops and Animal Products

In 80-km Radius Circle

Regional Productivity Factor:

Animal Grazing Factor:

Meteorology Perlod of Record: 1 MAY 74 - 30 APR 75 Recovery: 93\%
Veg: 2.0E7 kilogram

Milk: 2.6E8 liter

Meat: 1.6E7 kl logram

0.3

0.6

SITE SPECIFIC DATA - WATERBORNE PATHWAYS via CAPE COD BAY

Average Dilution F̧low

from Plant: $13 \mathrm{ft}^{3 / \mathrm{s}}$

Flsh:

Edible Harvest: $2.6 \mathrm{E} 4 \mathrm{~kg} / \mathrm{yr}$

Dilution Factor: 0.001

Invertebrates:

Edible Harvest: $3.1 E 4 \mathrm{~kg} / \mathrm{yr}$

Dilution Factor: 0.002 


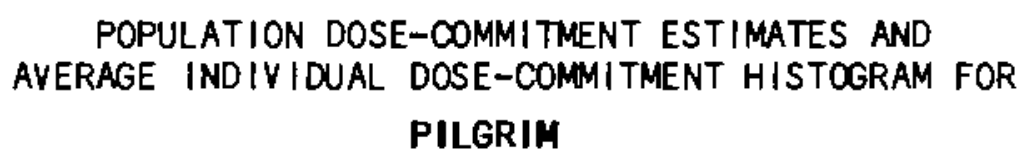

Dose Commitments (person-rem) from Liquid Pathways Iotal Body Gl-LLل Thyrold Bone

Liver

Infant

Chlld

Teen

Adult

TOTAL

Infant

Child

Teen

Adult

TOTAL
$0.0 \mathrm{E}+00$

4. $9 \mathrm{E}-03$

2. $8 \mathrm{E}-03$

$1.7 \mathrm{E}-02$

2. $4 \mathrm{E}-02$
$0.0 \mathrm{E}+00$

3. $5 \mathrm{E}-03$

$6.4 \mathrm{E}-03$

$5.1 \mathrm{E}-02$

6.1E-02

$0.0 \mathrm{E}+00$

$0.0 \mathrm{E}+00$

$0.0 \mathrm{E}+00$

2.2E-06

2.5E-02

$1.4 \mathrm{E}-02$

$1.0 \mathrm{E}-02$

$1.3 \mathrm{E}-05$

$1.4 \mathrm{E}-02$

6.0E-02

$1.7 \mathrm{E}-05$

$8.2 \mathrm{E}-02$

$8.4 \mathrm{E}-02$

Dose Commitments (person-rem) from Airborne Pathways

Total_Body

GJ-LLI Ihyrold

Bone

Lyer

Lung

$$
\text { 4. } 4 \mathrm{E}-02
$$

4. $4 \mathrm{E}-02$

9.3E-02

4. $4 \mathrm{E}-02$

4.5E-02

4.9E-01

$5.0 \mathrm{E}-01$

$3.6 \mathrm{E}-01$

3.6E-01

2. $2 \mathrm{E}+00$

2. $2 \mathrm{E}+00$

4.6E-02

3. $6 \mathrm{E}-01$

3. $6 \mathrm{E}-01$

5.2E-01

2. $2 \mathrm{E}+00$

2. $2 \mathrm{E}+00$

$2.8 \mathrm{E}+00$

$3.1 E+00$

$3.1 E+00$

$5.1 \mathrm{E}-01$

3. 8E-01

2.3E+00

3. $1 \mathrm{E}+00$

$3.1 E+00$

4. $2 \mathrm{E}+00$

$3.2 \mathrm{E}+00$

Production/Consumption factors:
Produce: <1
Milk: <1
Meat: <1

FRACTION OF POPULATION RECEIVING AN INDICATED AVERAGE

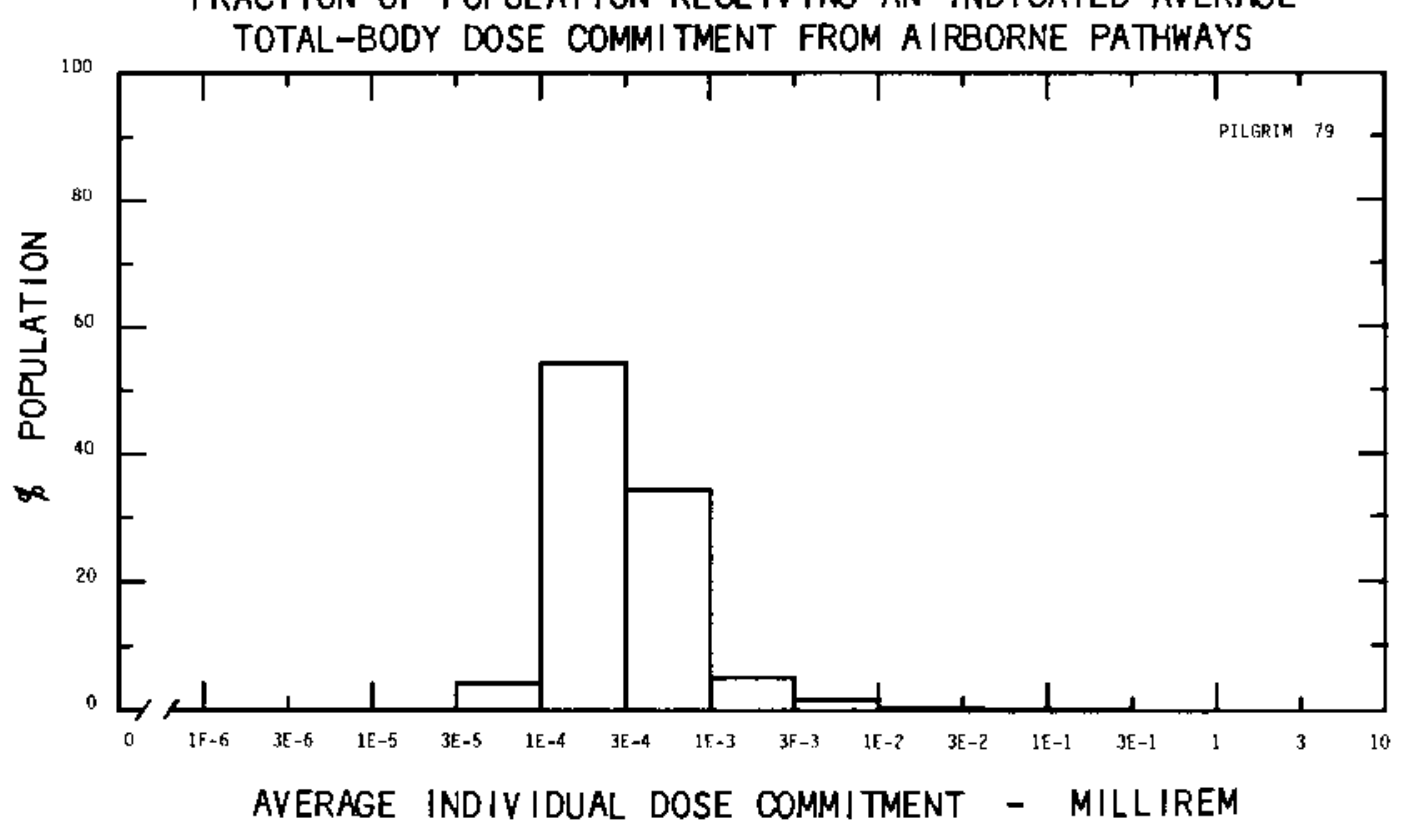


Slte: POINT BEACH

Location:
N $44.2764^{\circ}$
MNITOWOC COUNTY, WISCONSIN

W $87.5186^{\circ}$

POPULATION DATA

Total Population within 20-to-80-km Region: 6.0E5

Major Metropolitan Centers Within Region:

Center

Sheboygan

Manitowoc

Greenbay

Apploton
Population

52,000

36,000

94,000

61,000
Lecation

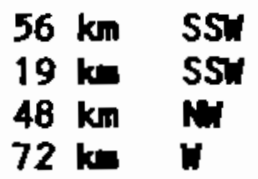

SITE SPECIFIC DATA - AIRBORNE PATHWAYS

Average Annual State Production

of Crops and Animal Products

In 80-km Radjus CIrcle

Reglonal Productivity Factor:

Animal Grazing Factor:
Veg: 7.2E7 kllogram

Milk: 1.2E9 liter

Meat: $1.0 E 8$ kllogram

0.5

0.5

Meteorology Period of Record: 19 APR 67 - 18 APR 69 Recovery: 83\%

SITE SPECIFIC DATA - WATERBORNE PATHWAYS via LAKE MIOHIGAN

$\begin{array}{ll} & \begin{array}{l}\text { Average DIlution Flow } \\ \text { from Plant: } 580 \mathrm{ft} / \mathrm{s}\end{array} \\ \text { Drinking Water: } & \text { Exposed Population: } 190,000 \\ & \text { Dilution Factor: } 2.6 \mathrm{E}-3 \mathrm{a}) \\ \text { Fish: } & \text { Edible Harvest: } 6.7 \mathrm{E4}(\mathrm{b}) \mathrm{kg} / \mathrm{yr} \\ & \text { Dilution Factor: } 0.013(\mathrm{c})\end{array}$

(a) Drinking water dllution factor estlmated by averaging dilution factor derived from FES (1972), sultably weighted for population.

(b) Includes both sport and commercial fish catch (FES, 1972).

(c) Dilution factor estimated by averaging sport and commerclal dilution factor derlved from FES (1972). 
POPULATION DOSE-COMMITMENT ESTIMATES AND AVERAGE INDIVIDUAL DOSE-COMMITMENT HISTOGRAM FOR

POINT BEACH 1 ND 2

Dose Commltments (person-rem) from Llquid Pathways

Total Body Gl-LLl Ihyrold Bene Lyer

$\begin{array}{llllll}\text { Infant } & 6.8 \mathrm{E}-04 & 6.7 \mathrm{E}-04 & 4.0 \mathrm{E}-03 & 1.0 \mathrm{E}-04 & 7.8 \mathrm{E}-04 \\ \text { ChIld } & \mathbf{8 . 4 \mathrm { E } - 0 3} & 7.7 \mathrm{E}-03 & 3.1 \mathrm{E}-02 & 5.3 \mathrm{E}-03 & 1.3 \mathrm{E}-02 \\ \text { Teen } & 4.4 \mathrm{E}-03 & 3.2 \mathrm{E}-03 & 1.0 \mathrm{E}-02 & 2.8 \mathrm{E}-03 & 7.0 \mathrm{E}-03 \\ \text { Adult } & 4.2 \mathrm{E}-02 & 2.7 \mathrm{E}-02 & 7.4 \mathrm{E}-02 & 1.6 \mathrm{E}-02 & 4.9 \mathrm{E}-02 \\ \text { TOTAL } & 5.5 \mathrm{E}-02 & 3.9 \mathrm{E}-02 & 1.2 \mathrm{E}-01 & 2.5 \mathrm{E}-02 & 7.0 \mathrm{E}-02\end{array}$

Dose Commltments (person-rem) from Alrborne Pathways

IotalBody Gl-LLl Thyrold Bone Llver Lung

$\begin{array}{lllllll}\text { Infant } & 2.6 \mathrm{E}-03 & 2.6 \mathrm{E}-03 & 3.8 \mathrm{E}-03 & 5.9 \mathrm{E}-04 & 2.7 \mathrm{E}-03 & 2.7 \mathrm{E}-03 \\ \text { Child } & 4.0 \mathrm{E}-02 & 4.0 \mathrm{E}-02 & 4.8 \mathrm{E}-02 & 6.5 \mathrm{E}-03 & 4.1 \mathrm{E}-02 & 4.0 \mathrm{E}-02 \\ \text { Teen } & 2.5 \mathrm{E}-02 & 2.4 \mathrm{E}-02 & 2.8 \mathrm{E}-02 & 4.3 \mathrm{E}-03 & 2.5 \mathrm{E}-02 & 2.5 \mathrm{E}-02 \\ \text { Adult } & 1.4 \mathrm{E}-01 & 1.4 \mathrm{E}-01 & 1.5 \mathrm{E}-01 & 2.5 \mathrm{E}-02 & 1.4 \mathrm{E}-01 & 1.4 \mathrm{E}-01 \\ \text { TOTAL } & 2.0 \mathrm{E}-01 & 2.0 \mathrm{E}-01 & 2.3 \mathrm{E}-01 & 3.7 \mathrm{E}-02 & 2.0 \mathrm{E}-01 & 2.0 \mathrm{E}-01\end{array}$

Production/Consumption factors:

Produce: <1 Milk: 7.5 Meat: 1.1

FRACTION OF POPULATION RECEIVING AN INDICATED AVERAGE TOTAL-BODY DOSE COMMITMENT FROM AIRBORNE PATHWAYS

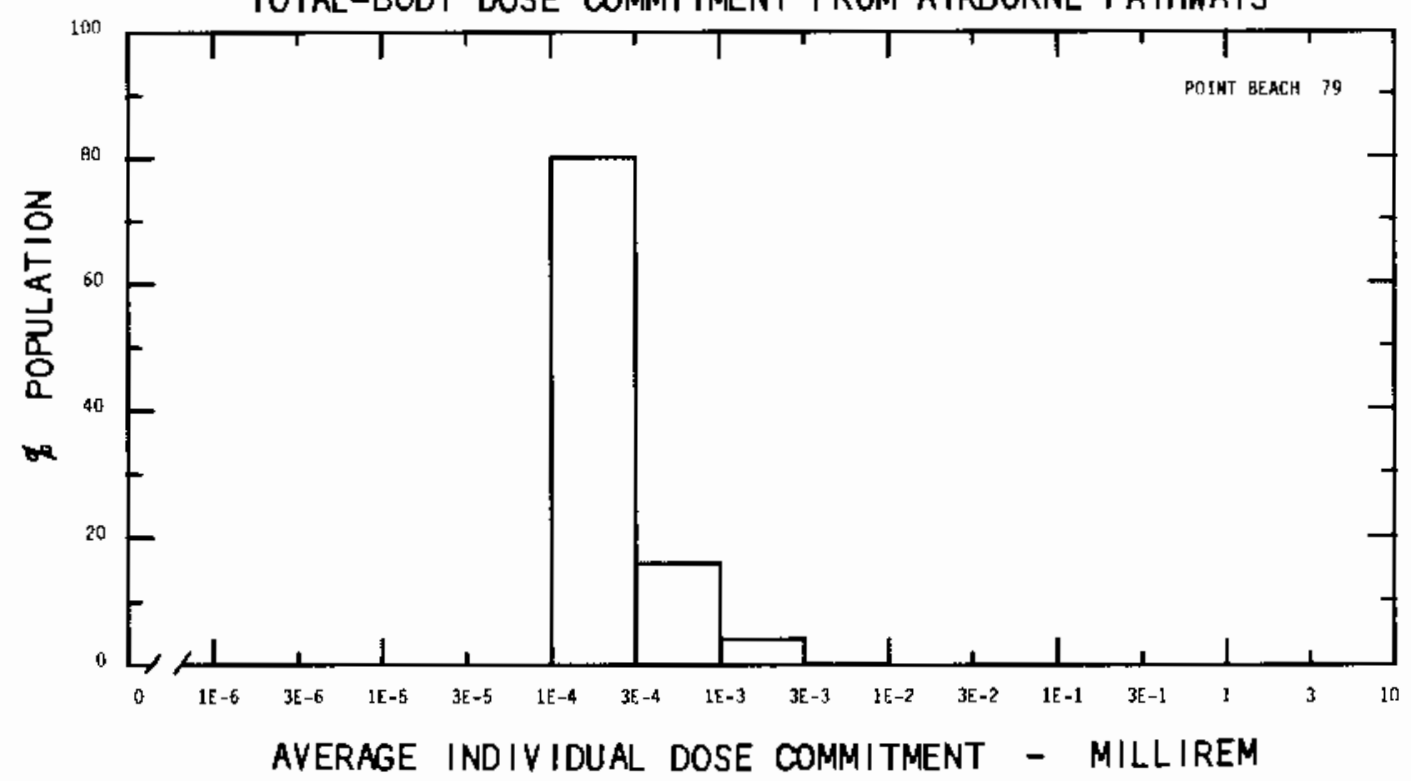


SIte: PRAIRIE ISLND

Lecation: N $44.6217^{\circ}$
RED WING, MIMNESOTA

W $92.6330^{\circ}$

POPULATION DATA

Total Population with in 20-to-80-km Reglon: 2.2E6

Major Metropolitan Centers Within Region:

Center

Minneapol Is-St. Paul SHSA

Rochester

Hastings
Population

2.1E6

57,000

13,000
Lecation

$50 \mathrm{~km} \quad \mathrm{Nm}$

$64 \mathrm{~km}$ SSE

$19 \mathrm{~km} \mathrm{Ni}$

SITE SPECIFIC DATA - AIRBORNE PATHWAYS

Average Annual State Production

of Crops and Anlmal Products

In 80-km Radius Circle

Regional Productivity Factor:

Animal Grazing Factor:

Meteorology Period of Record: 22 MAR 74 - 21 MR 75 Recovery: 65\%

SITE SPECIFIC DATA - WATEPBORNE PATHWAYS VIO MISSISSIPPI RIVER

Average River Flow

at Site: $15,000 \mathrm{ft}^{3} / \mathrm{s}$

Drinking Water:

Exposed Population: None

Fish:

Edlble Harvest: $6.8 E 5 \mathrm{~kg} / \mathrm{yr}$

Dllution Factor: 1 


\section{POPULATION DOSE-COMMITMENT ESTIMATES AND \\ AVERAGE INDIVIDUAL DOSE-COMMITMENT HISTOGRAM FOR \\ PRAIRIE ISLAND 1 ND 2}

Dose Commitments (person-rem) from Liquid Pathways

Iotal Body GL-LLI Ihyrold Bone Llver

$\begin{array}{llllll}\text { Infant } & 0.0 \mathrm{E}+00 & 0.0 \mathrm{E}+00 & 0.0 \mathrm{E}+00 & 0.0 \mathrm{E}+00 & 0.0 \mathrm{E}+00 \\ \text { Child } & 3.6 \mathrm{E}-04 & 3.7 \mathrm{E}-04 & 4.6 \mathrm{E}-04 & 1.9 \mathrm{E}-05 & 3.7 \mathrm{E}-04 \\ \text { Teen } & 3.3 \mathrm{E}-04 & 3.5 \mathrm{E}-04 & 3.9 \mathrm{E}-04 & 1.1 \mathrm{E}-05 & 3.4 \mathrm{E}-04 \\ \text { Adult } & 3.6 \mathrm{E}-03 & 2.9 \mathrm{E}-03 & 3.0 \mathrm{E}-03 & 6.0 \mathrm{E}-05 & 2.6 \mathrm{E}-03 \\ \text { TOTAL } & 3.3 \mathrm{E}-03 & 3.6 \mathrm{E}-03 & 3.9 \mathrm{E}-03 & 9.6 \mathrm{E}-05 & 3.3 \mathrm{E}-03\end{array}$

Dose Commitments (person-rem) from Airborne Pathways

TotalBody GJ-LLI Ihyrold Bone Llver Lung

$\begin{array}{lllllll}\text { Infant } & 1.3 \mathrm{E}-03 & 1.3 \mathrm{E}-03 & 6.7 \mathrm{E}-03 & 3.3 \mathrm{E}-04 & 1.3 \mathrm{E}-03 & 1.3 \mathrm{E}-03 \\ \text { ChIld } & 1.9 \mathrm{E}-02 & 1.9 \mathrm{E}-02 & 5.4 \mathrm{E}-02 & 3.7 \mathrm{E}-03 & 1.9 \mathrm{E}-02 & 1.9 \mathrm{E}-02 \\ \text { Teen } & 1.2 \mathrm{E}-02 & 1.2 \mathrm{E}-02 & 2.6 \mathrm{E}-02 & 2.6 \mathrm{E}-03 & 1.2 \mathrm{E}-02 & 1.3 \mathrm{E}-02 \\ \text { Adult } & 6.8 \mathrm{E}-02 & 6.8 \mathrm{E}-02 & 1.1 \mathrm{E}-01 & 1.6 \mathrm{E}-02 & 6.8 \mathrm{E}-02 & 6.9 \mathrm{E}-02 \\ \text { TOTAL } & 1.0 \mathrm{E}-01 & 1.0 \mathrm{E}-01 & 2.0 \mathrm{E}-01 & 2.2 \mathrm{E}-02 & 1.0 \mathrm{E}-01 & 1.0 \mathrm{E}-01\end{array}$

Production/Consumption factors:

Produce: <1 Milk: $1.4 \quad$ Meat: <1

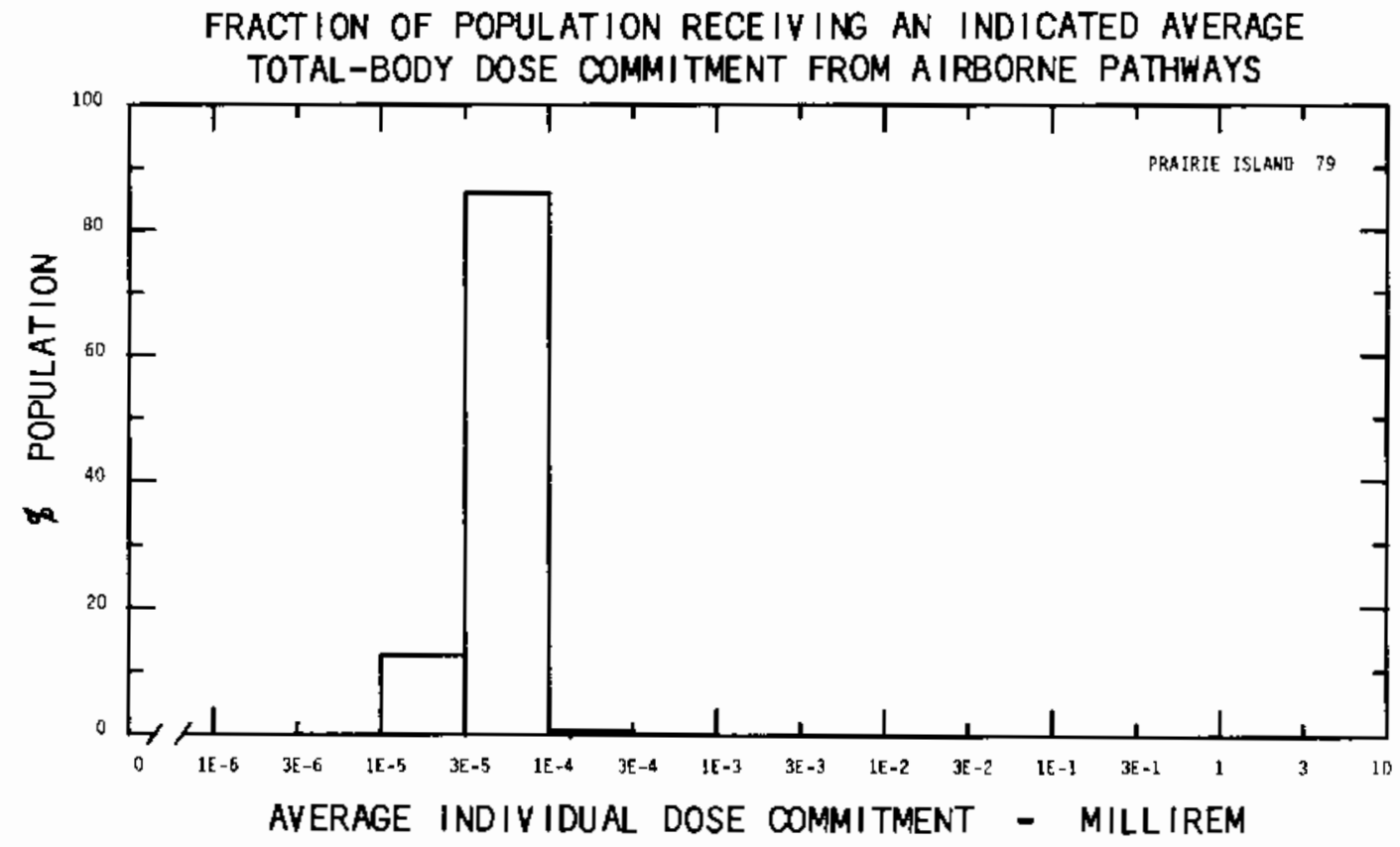


SIte: QUAD CITJES

Lecation: $\quad$ N $41.7272^{\circ}$
ROCK ISLAND, ILLINOIS

W $90.3417^{\circ}$

POPULATION DATA

Total Population Within 20-to-80-km Reglon: 6.7E5

Major Metropolitan Centers Within Reglon:

Center Ropulation Lecation

Davenport SMSA $\quad 370,000 \quad 40 \mathrm{~km} \quad$ Sit

SITE SPECIFIC DATA - AIRBORNE PATHWAYS

Average Annual State Production

of Crops and Animal Products

In 80-km Radius Circle

Regional Productivity Factor:

Animal GrazIng Factor:

Meteorology Period of Record: 1 JN 74 - 31 DEC 75 Recovery: 88\%
Veg: 1.1E8 kilogram

MIlk: 1.8E8 I iter

Meat: 1.9E8 kilogram

1

0.5

SITE SPECIFIC DATA - WATERBORNE PATHWAYS VIa MISSISSIPPI RIVER
Average River Flow
at Site: $47,000 \mathrm{ft}^{3} / \mathrm{s}$
Drinking Water:
Exposed Population: $370,000(a)$
Dilution Factor: 1
Fish:
Edible Harvest: $2.16^{(b)} \mathrm{kg} / \mathrm{yr}$
Dilution Factor: $0.5^{(b)}$

(a) All people in Davenport SMSA assumed to or Ink river water.

(b) Assumes $1 / 2 \mathrm{fish}$ harvest caught below plant. 
POPULATION DOSE-COMMITMENT ESTIMATES AND

AVERAGE INDIVIDUAL DOSE-COMMITMENT HISTOGRAM FOR

\section{QUAD CITIES 1 ND 2}

Dose Commitments (person-rem) from Liquid Pathways IotalBody Gl-LLl Thyrold Bone Llver

Infant

Child

Teen

Adult

TOTAL
2.3E-03

$1.3 \mathrm{E}=01$

2.0E-01

2. $2 \mathrm{E}+00$

$2.5 \mathrm{E}+00$
$1.1 E-03$

2.3E-02

2.3E-02

$1.9 \mathrm{E}-01$

2. 4E-01
3.2E-02

2.3E-01

7.4E-02

5.2E-01

8.5E-01
3. $6 \mathrm{E}-02$

8. $4 \mathrm{E}-01$

3.9E-01

$2.3 E+00$

$3.6 \mathrm{E}+00$
$7.6 \mathrm{E}-03$

6. $1 \mathrm{E}-01$

4. $9 E-01$

$2.9 \mathrm{E}+00$

$4.0 \mathrm{E}+00$

Dose Commltments (person-rem) from Alrborne Pathways

TotalBody Gl-Lلl Ihyrold Bone Llver Lung

$\begin{array}{lllllll}\text { Infant } & 5.6 \mathrm{E}-02 & 5.6 \mathrm{E}-02 & 3.6 \mathrm{E}-01 & 5.8 \mathrm{E}-02 & 5.7 \mathrm{E}-02 & 5.8 \mathrm{E}-02 \\ \text { Child } & 6.5 \mathrm{E}-01 & 6.3 \mathrm{E}-01 & 3.7 \mathrm{E}+00 & 8.3 \mathrm{E}-01 & 6.4 \mathrm{E}-01 & 6.5 \mathrm{E}-01 \\ \text { Teen } & 4.6 \mathrm{E}-01 & 4.7 \mathrm{E}-01 & 1.7 \mathrm{E}+00 & 5.2 \mathrm{E}-01 & 4.6 \mathrm{E}-01 & 4.9 \mathrm{E}-01 \\ \text { Adult } & 2.8 \mathrm{E}+00 & 2.8 \mathrm{E}+00 & 7.4 \mathrm{E}+00 & 3.0 \mathrm{E}+00 & 2.8 \mathrm{E}+00 & 2.9 \mathrm{E}+0 \mathrm{D} \\ \text { TOTAL } & 4.0 \mathrm{E}+00 & 4.0 \mathrm{E}+00 & 1.3 \mathrm{E}+01 & 4.4 \mathrm{E}+00 & 3.9 \mathrm{E}+00 & 4.1 \mathrm{E}+00\end{array}$

Production/Consumption factors:
Produce: $<1$
Milk: 2.0
Meat: 3.5

FRACTION OF POPULATION RECEIVING AN INDICATED AVERAGE TOTAL-BODY DOSE COMMITMENT FROM AIRBORNE PATHWAYS

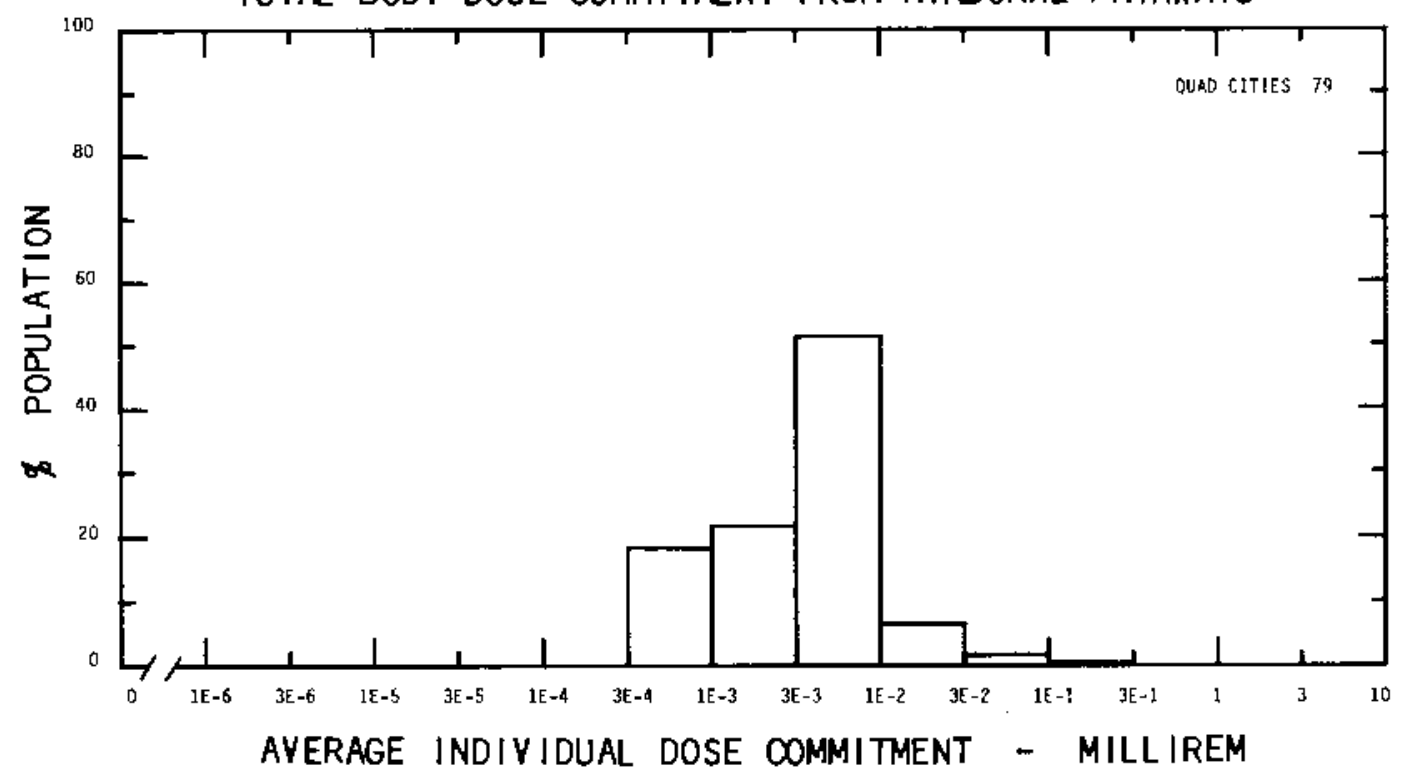


SIte: RANCHO SECO

Lecation:
SACRAEETTO COUNTY, CALIFORNIA

W $121.1186^{\circ}$

POPULATION DATA

Total Population within 20-to-80-km Region: 1.6E6

Major Metropolitan Centers Within Region:

Center

Secremento SUSA

Stockton
Population

900,000

120,000
Location

$35 \mathrm{~km} \quad$ NMN

$45 \mathrm{~km} \mathrm{SW}$

SITE SPECIFIC DATA - AIRBORNE PATHWAYS

Average Annual State Production

of Crops and AnImal Products

In 80-km Radius Clrcle

Regional Productivity Factor:

AnImal Grazing Factor:

Meteorology Perlod of Record: 1 FEB 75 - 31 JAN 76 Recovery: 98\$
Veg: 4.8E7 kilogram

Mllk: 2.3E8 liter

Meat: $5.0 \mathrm{E7}$ kllogram

1

0.9

SITE SPECIFIC DATA - WATERBORNE PATHWAYS

$\begin{array}{ll} & \begin{array}{l}\text { Average Dilution Flow } \\ \text { from Plant: (a) }\end{array} \\ \text { Drinking Water: } & \text { Exposed Population: (a) } \\ \text { Fish: } & \text { Edible Harvest: (a) }\end{array}$

(a) No radionuclides released in liquid effluents reported (Tichler, et al., 1981). 


\section{POPULATION DOSE-COMMITMENT ESTIMATES AND AVERAGE INDIVIDUAL DOSE-COMMITMENT HISTOGRAM FOR RANCHO SECO}

Dose Commitments (person-rem)
$\begin{array}{llll}\text { Iotal Body } & \text { Gl-LLl } & \text { Thyrold } & \text { Bone }\end{array}$

No Liquid Pathway Doses

Dose Commitments (person-rem) from Airborne Pathways

Iotal Body GL-LLl Ihyrold Bone Liver Lung

$\begin{array}{lllllll}\text { Infant } & 4.7 \mathrm{E}-03 & 4.7 \mathrm{E}-03 & 9.9 \mathrm{E}-03 & 3.9 \mathrm{E}-03 & 4.7 \mathrm{E}-03 & 4.9 \mathrm{E}-03 \\ \text { Child } & 5.4 \mathrm{E}-02 & 5.4 \mathrm{E}-02 & 8.2 \mathrm{E}-02 & 4.3 \mathrm{E}-02 & 5.4 \mathrm{E}-02 & 5.8 \mathrm{E}-02 \\ \text { Teen } & 3.8 \mathrm{E}-02 & 3.8 \mathrm{E}-02 & 4.9 \mathrm{E}-02 & 3.1 \mathrm{E}-02 & 3.8 \mathrm{E}-02 & 4.3 \mathrm{E}-02 \\ \text { Adult } & 2.3 \mathrm{E}-01 & 2.3 \mathrm{E}-01 & 2.6 \mathrm{E}-01 & 1.9 \mathrm{E}-01 & 2.3 \mathrm{E}-01 & 2.4 \mathrm{E}-01 \\ \text { TOTAL } & 3.2 \mathrm{E}-01 & 3.2 \mathrm{E}-01 & 4.0 \mathrm{E}-01 & 2.7 \mathrm{E}-01 & 3.2 \mathrm{E}-01 & 3.5 \mathrm{E}-01\end{array}$

Production/Consumption factors:

Produce: <1 Milk: $1.2 \quad$ Meat: <1

FRACTION OF POPULATION RECEIVING AN INDICATED AVERAGE

TOTAL-BODY DOSE COMMITMENT FROM AIRBORNE PATHWAYS

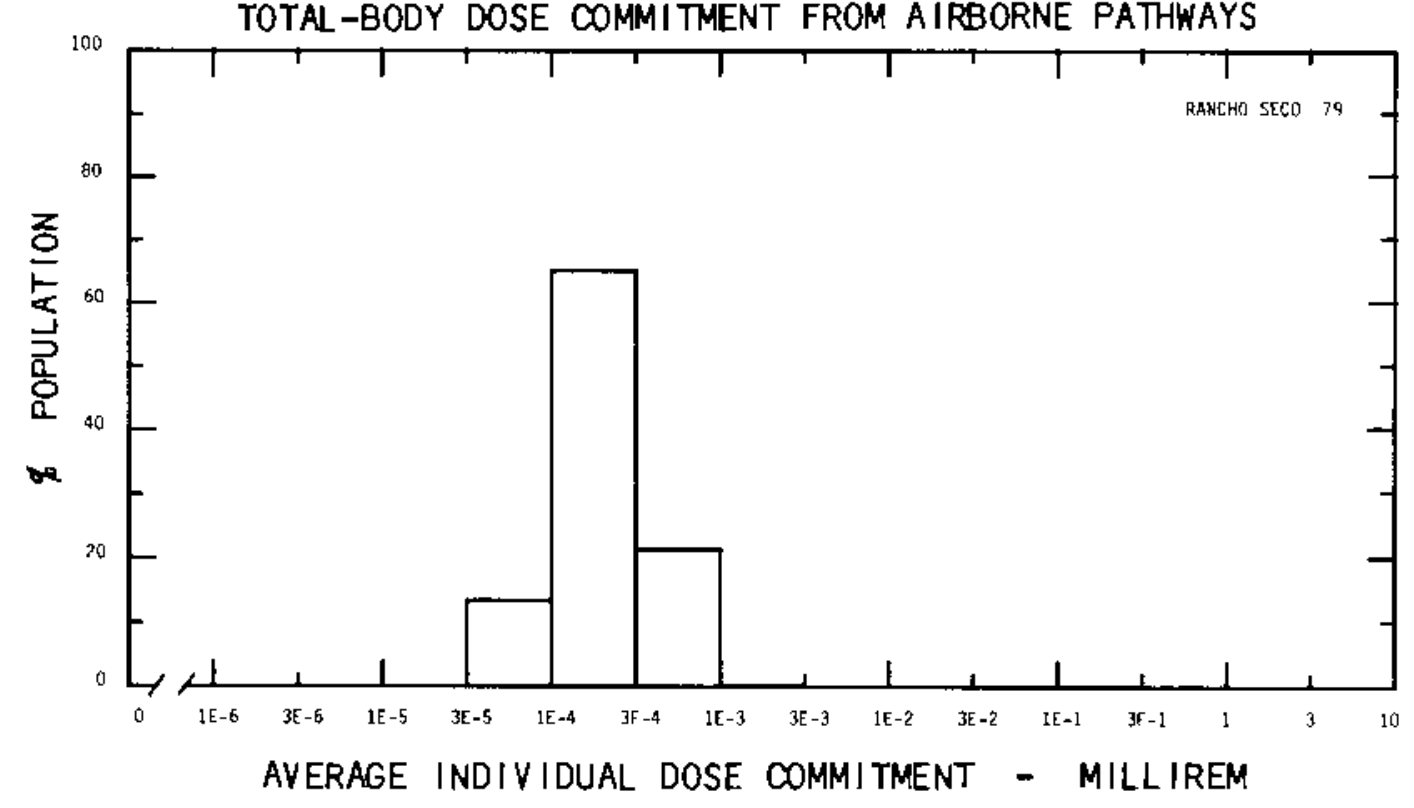


Site: H. B. ROBINSON

Lecation:

N $34.4014^{\circ}$

HARTSY ILLE, SOUTH CAROL INU

W $80.1567^{\circ}$

Total Population Within 20-to-80-km Region: 6.5E5

Major Metropolitan Centers Within Region:

Center

Florence

Sumter
Population

29,000

28,000
Location

$40 \mathrm{~km} \quad \mathrm{SE}$

$53 \mathrm{~km} \quad$ SSW

SITE SPECIFIC DATA - AIRBORNE PATHWAYS

Average Annual State Production

Of Crops and Animal Products

In 80-km Radlus Circle

Regional Productivity Factor:

Animal Grazing Factor:

Meteorology Period of Record: 1 JAN 75 - 31 DEC 75 Recovery: 94\%
Veg: 7.4E6 kllogram

Milk: 5.7E7 Ilter

Meat: $5.0 \mathrm{E7}$ kilogram

1

0.8

SITE SPECIFIC DATA - WATERBORNE PATHWAYS via LAKE ROBINSON

Average Dilution Flow

from Plant: $960 \mathrm{ft} / \mathrm{s}$

Drinking Water:

Exposed Population: None

Fish:

Edible Harvest: $1.8^{(a)} \mathrm{kg} / \mathrm{yr}$
Dilution Factor: 0.02

(a) Average Individual consumption rate as given in the FES (1975) used in lieu of catch data.

b) Ten percent of population consumes fish taken from water diluted by a factor of 0.2 (FES, 1975). 
POPULATION DOSE-COMMITMENT ESTIMATES AND AVERAGE INDIVIDUAL DOSE-COMMITMENT HISTOGRAM FOR

\section{H. B. ROBINSON}

Dose Commitments (person-rem) from Liquid Pathways

Iotal Body GJ=LL Ihyrold Bone Llver

$\begin{array}{llllll}\text { Infant } & 0.0 \mathrm{E}+00 & 0.0 \mathrm{E}+00 & 0.0 \mathrm{E}+00 & 0.0 \mathrm{E}+00 & 0.0 \mathrm{E}+00 \\ \text { ChIld } & 1.0 \mathrm{E}-02 & 1.0 \mathrm{E}-03 & 4.1 \mathrm{E}-04 & 3.7 \mathrm{E}-02 & 4.2 \mathrm{E}-02 \\ \text { Teen } & 1.5 \mathrm{E}-02 & 2.0 \mathrm{E}-03 & 3.2 \mathrm{E}-04 & 2.2 \mathrm{E}-02 & 3.6 \mathrm{E}-02 \\ \text { Adult } & 1.5 \mathrm{E}-01 & 1.8 \mathrm{E}-02 & 2.3 \mathrm{E}-03 & 1.3 \mathrm{E}-01 & 2.1 \mathrm{E}-01 \\ \text { TOTAL } & 1.7 \mathrm{E}-01 & 2.1 \mathrm{E}-02 & 3.0 \mathrm{E}-03 & 1.9 \mathrm{E}-01 & 2.9 \mathrm{E}-01\end{array}$

Dose Commitments (person-rem) from Alrborne Pathways

Iotal Body GJ=LL Ihyrold Bone Llver Lung

$\begin{array}{lllllll}\text { Infant } & 3.4 \mathrm{E}-04 & 3.4 \mathrm{E}-04 & 3.4 \mathrm{E}-04 & 3.1 \mathrm{E}-04 & 3.4 \mathrm{E}-04 & 3.7 \mathrm{E}-04 \\ \text { Child } & 3.9 \mathrm{E}-03 & 3.9 \mathrm{E}-03 & 3.9 \mathrm{E}-02 & 3.5 \mathrm{E}-03 & 3.9 \mathrm{E}-03 & 4.3 \mathrm{E}-03 \\ \text { Teen } & 2.8 \mathrm{E}-03 & 2.8 \mathrm{E}-03 & 2.8 \mathrm{E}-03 & 2.5 \mathrm{E}-03 & 2.8 \mathrm{E}-03 & 3.4 \mathrm{E}-03 \\ \text { AduIt } & 1.7 \mathrm{E}-02 & 1.7 \mathrm{E}-02 & 1.7 \mathrm{E}-02 & 1.5 \mathrm{E}-02 & 1.7 \mathrm{E}-02 & 1.9 \mathrm{E}-02 \\ \text { TOTAL } & 2.4 \mathrm{E}-02 & 2.4 \mathrm{E}-02 & 2.4 \mathrm{E}-02 & 2.2 \mathrm{E}-02 & 2.4 \mathrm{E}-02 & 2.7 \mathrm{E}-02\end{array}$

Production/Consumption factors:

Produce: $<1 \quad$ Milk: $<1 \quad$ Meat: $<1$

FRACTION OF POPULATION RECEIVING AN INDICATED AVERAGE TOTAL-BODY DOSE COMMITMENT FROM AIRBORNE PATHWAYS

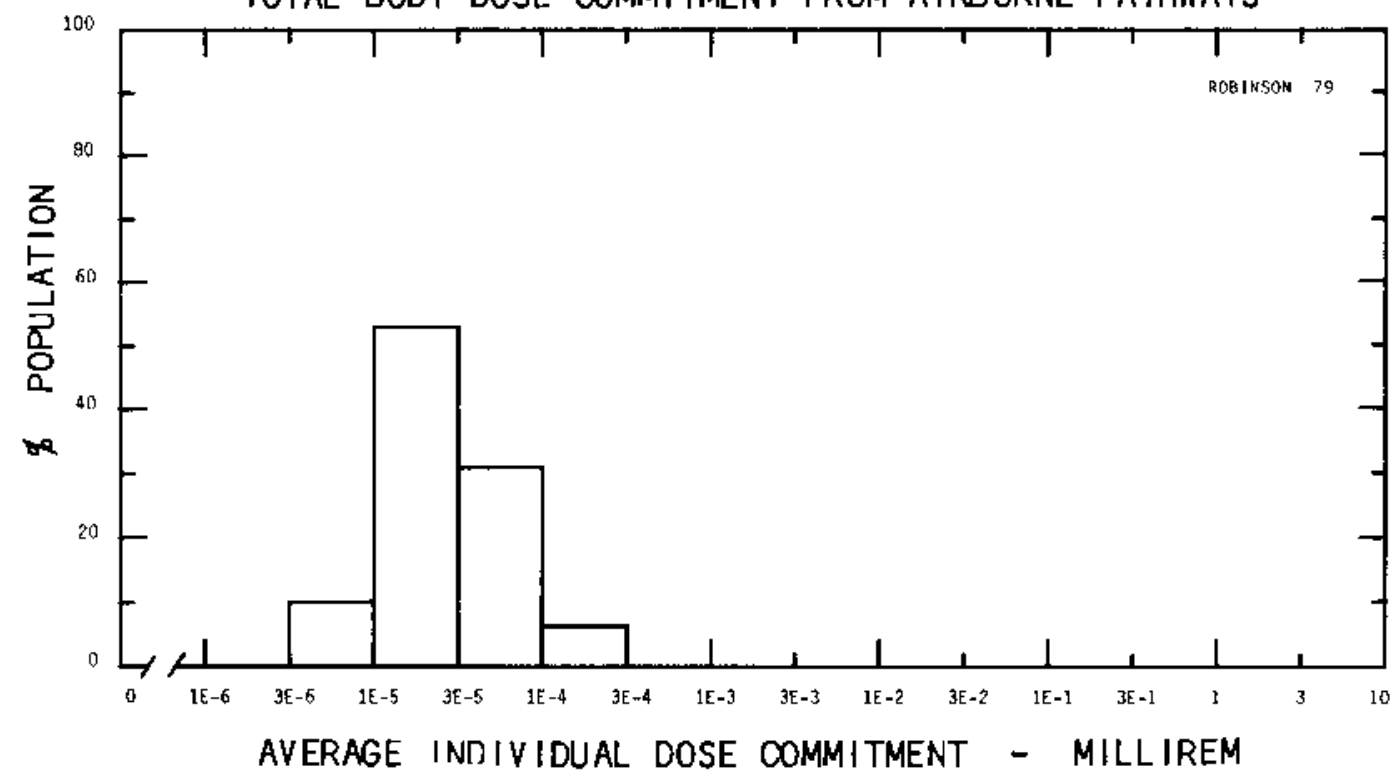


SIte: ST. LUCIE

FCRT PIERCE, FLORIDA

Location: N $27.4466^{\circ}$ W $80.3266^{\circ}$

POPULATION DATA

Total Population Within 20-to-80-km Region: 3.0E5

Major Metropolitan Centers Within Region:

Center

West Palm Beach

Ft. Plerce

Vero Beach
Population

75,000

38,000

16,000
Location

$80 \mathrm{kn} \quad \mathrm{SSE}$

$13 \mathrm{~km}$

$40 \mathrm{~km}$

SITE SPECIFIC DATA - AIRBORNE PATHWAYS

Average Annual State Production

of Crops and Animal Products

In 80-km Radius Circle

Reglonal Productivity Factor:

Animal Grazing Factor:

Meteorology Perlod of Record: 1 JAN 76 - 31 DEC 76 Recovery: 92\%
Veg: 2.8E7 kilogram

Milk: 1.1E8 liter

Meat: 7.2E7 kllogram

0.5

1

SITE SPECIFIC DATA - WATERBORNE PATHWAYS VIa ATLANTIC OCEAN

Average Dilution Elow

from Plant: $35 \mathrm{ft}^{3 / \mathrm{s}}$

Fish:

Edible Harvest: $2.6 \mathrm{E5} \mathrm{kg} / \mathrm{yr}$
Dilution Factor: $0.005 \mathrm{~g})$

Invertebrates:

Edible Harvest: $2.764 \mathrm{~kg} / \mathrm{yr}$

(a) Dilution factors as given in FES (1973). 
POPULATION DOSE-COMMITMENT ESTIMATES AND

AVERAGE INDIVIDUAL DOSE-COMMITMENT HISTOGRAM FOR

\section{SAINT LUCIE}

Dose Commitments (person-rem) from Liquld Pathways

Total Body GLLLl Thyredd Bone Llyer

$\begin{array}{llllll}\text { Infant } & 0.0 \mathrm{E}+00 & 0.0 \mathrm{E}+00 & 0.0 \mathrm{E}+00 & 0.0 \mathrm{E}+00 & 0.0 \mathrm{E}+00 \\ \text { Child } & 1.2 \mathrm{E}-02 & 5.4 \mathrm{E}-02 & 5.9 \mathrm{E}-03 & 2.3 \mathrm{E}-02 & 3.2 \mathrm{E}-02 \\ \text { Teen } & 1.4 \mathrm{E}-02 & 1.1 \mathrm{E}-01 & 4.2 \mathrm{E}-03 & 1.4 \mathrm{E}-02 & 2.8 \mathrm{E}-02 \\ \text { Adult } & 1.2 \mathrm{E}-01 & 1.0 \mathrm{E}+00 & 2.8 \mathrm{E}-02 & 8.2 \mathrm{E}-02 & 1.7 \mathrm{E}-01 \\ \text { TOTAL } & 1.5 \mathrm{E}-01 & 1.2 \mathrm{E}+00 & 3.8 \mathrm{E}-02 & 1.2 \mathrm{E}-01 & 2.2 \mathrm{E}-01\end{array}$

Dose Commitments (person-rem) from Airborne Pathways

Total Body GL-LLI Thyrodd Bone LIver Lung

$\begin{array}{lllllll}\text { Infant } & 8.5 \mathrm{E}-03 & 8.5 \mathrm{E}-03 & 4.4 \mathrm{E}-02 & 7.9 \mathrm{E}-03 & 8.6 \mathrm{E}-03 & 8.9 \mathrm{E}-03 \\ \text { ChIld } & 9.9 \mathrm{E}-02 & 9.9 \mathrm{E}-02 & 3.5 \mathrm{E}-01 & 8.7 \mathrm{E}-02 & 9.9 \mathrm{E}-02 & 1.0 \mathrm{E}-01 \\ \text { Teen } & 7.2 \mathrm{E}-02 & 7.2 \mathrm{E}-02 & 1.9 \mathrm{E}-01 & 6.4 \mathrm{E}-02 & 7.2 \mathrm{E}-02 & 7.9 \mathrm{E}-02 \\ \text { Adult } & 4.3 \mathrm{E}-01 & 4.3 \mathrm{E}-01 & 9.0 \mathrm{E}-01 & 3.9 \mathrm{E}-01 & 4.4 \mathrm{E}-01 & 4.6 \mathrm{E}-01 \\ \text { TOTAL } & 6.1 \mathrm{E}-01 & 6.1 \mathrm{E}-01 & 1.5 \mathrm{E}+00 & 5.4 \mathrm{E}-01 & 6.2 \mathrm{E}-01 & 6.5 \mathrm{E}-01\end{array}$

Production/Consumption factors:

Produce: $<1$ Milk: 1.4 Meat: 1.5

FRACTION OF POPULATION RECEIVING AN INDICATED AVERAGE TOTAL-BODY DOSE COMMITMENT FROM AIRBORNE PATHWAYS

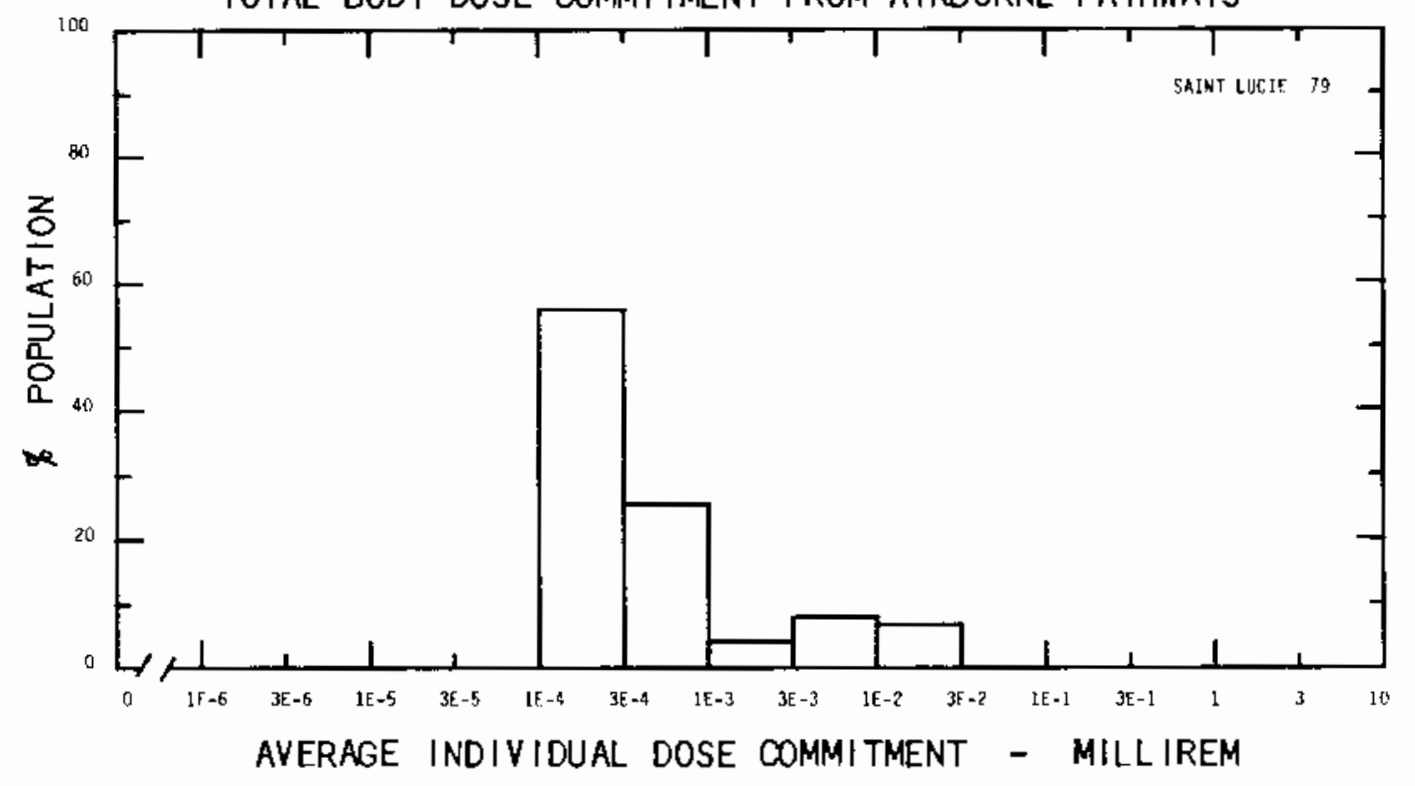


SIte: SNEM

SNEM, MEN JERSEY

Lecation: $\quad N 39.4628^{\circ}$ W $75.5356^{\circ}$

POPULATION DATA

Total Population within 20-to-80-km Reglon: 4.9E6

Major Metropolitan Centers Within Region:

Center

PhIladelphla SMSA

WIIrington SISA

Chester
Population

$4,900,000$

510,000

58,000
Location

$64 \mathrm{~km} \quad \mathrm{ME}$

$29 \mathrm{~km} \quad \mathrm{~N}$

$45 \mathrm{~km}$ NNE

SITE SPECIFIC DATA - AIRBORNE PATHWAYS

Average Annual State Production

of Crops and Animal Products

in 80-km Radius Circle

Regional Productivity Factor:

Animal Grazlng Factor:
Veg: 7.4E7 kilogram

MIIK: 2.7EB liter

Meat: 2.4E7 kilogram

0.9

0.6

Meteorology Perlod of Record: 1 JUN 70 - 31 MAY 71 Recovery: 95\%

SITE SPECIFIC DATA - WATERBORNE PATHWAYS VIa DELAWARE RIVER ESTUARY

Average River Flow

at Site: $16,000 \mathrm{ft}^{3} / \mathrm{s}$

Fish:

Edible Harvest: $3.6 E 5 \mathrm{~kg} / \mathrm{yr}$

Dilution Factor: 1

Invertebrates: $(a)$

Edible Harvest: $1.6 E 5 \mathrm{~kg} / \mathrm{yr}$

Dilution Factor: 1

(a) Environment primarlly salt water so invertebrates considered in I ieu of drinking water. 
POPULATION DOSE-COMMITMENT ESTIMATES AND AVERAGE INDIVIDUAL DOSE-COMMITMENT HISTOGRAM FOR

\section{SNEM}

Dose Cormitments (person-rem) from Llquid Pathways Iotal Body $\mathrm{Gl-Lلl}$ Ihyrold Bone Llver

Infant Child

Teen

Adult

TOTAL

$0.0 \mathrm{E}+00$
$3.8 \mathrm{E}-02$
$2.6 \mathrm{E}-02$
$1.6 \mathrm{E}-01$
$2.3 \mathrm{E}-01$

$0.0 \mathrm{E}+00$
$7.3 \mathrm{E}-02$
$1.5 \mathrm{E}-01$
$1.3 \mathrm{E}+00$
$1.6 \mathrm{E}+00$

$0.0 \mathrm{E}+00$

$0.0 \mathrm{E}+00$

$0.0 \mathrm{E}+00$

$3.2 \mathrm{E}-03$

9.1E-03

2.3E -03

$5.4 \mathrm{E}-03$

2.9E-02

$1.5 \mathrm{E}-02$

$3.2 \mathrm{E}-02$

$2.6 \mathrm{E}-02$

$2.1 \mathrm{E}-02$

$4.7 \mathrm{E}-02$

$1.6 \mathrm{E}-01$

2.1E-01

Dose Commitments (person-rem) from Airborne Pathways

Iotal Body Gl-Lلl Ihyrold Bone Llver Lung

$\begin{array}{lllllll}\text { Infant } & 2.6 \mathrm{E}-02 & 2.6 \mathrm{E}-02 & 3.2 \mathrm{E}-02 & 2.7 \mathrm{E}-04 & 2.6 \mathrm{E}-02 & 2.6 \mathrm{E}-02 \\ \text { Child } & 3.8 \mathrm{E}-01 & 3.8 \mathrm{E}-01 & 4.2 \mathrm{E}-01 & 2.9 \mathrm{E}-03 & 3.8 \mathrm{E}-01 & 3.8 \mathrm{E}-01 \\ \text { Teen } & 2.6 \mathrm{E}-01 & 2.6 \mathrm{E}-01 & 2.7 \mathrm{E}-01 & 2.1 \mathrm{E}-03 & 2.6 \mathrm{E}-01 & 2.6 \mathrm{E}-01 \\ \text { Adult } & 1.4 \mathrm{E}+00 & 1.4 \mathrm{E}+00 & 1.5 \mathrm{E}+00 & 1.3 \mathrm{E}-02 & 1.4 \mathrm{E}+00 & 1.4 \mathrm{E}+00 \\ \text { TOTAL } & 2.1 \mathrm{E}+00 & 2.1 \mathrm{E}+00 & 2.2 \mathrm{E}+00 & 1.8 \mathrm{E}-02 & 2.1 \mathrm{E}+00 & 2.1 \mathrm{E}+00\end{array}$

\section{Production/Consumption factors:}

Produce: <1 Milk: <1 Meat: <1

FRACTION OF POPULATION RECEIVING AN INDICATED AVERAGE TOTAL-BODY DOSE COMMITMENT FROM AIRBORNE PATHWAYS

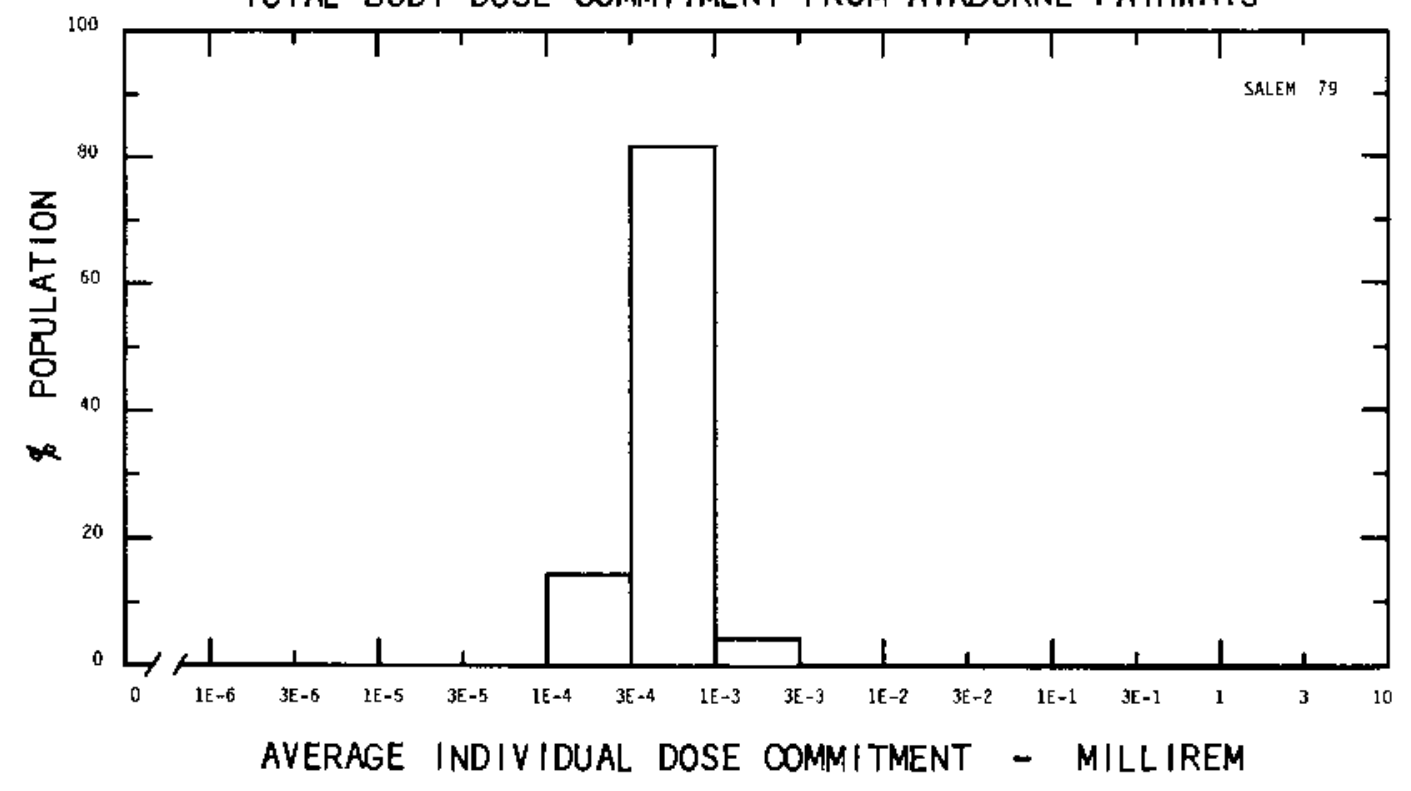


SIte: SAN OMOFRE

Lecation: N $33.3686^{\circ}$
CAPP PEKDLETON, CN IFCRNIA

W $117.5544^{\circ}$

POPULATION DATA

Total Population within 20-to-80-km Region: 4.0E6

Major Metropolitan Centers Within Region:

Center

San Dlego SMSA

Riverside

Anaheim

Santa Ana
Population

$1,500,000$

160,000

190,000

180,000
Lecation

$68 \mathrm{kn}$ SSE

$69 \mathrm{~km} \quad \mathrm{NE}$

$69 \mathrm{~km} \mathrm{NBH}$

$55 \mathrm{~km}$ NMY

SITE SPECIFIC DATA - AIRBORNE PATHWAYS

Average Annual State Production

of Crops and Animal Products

In 80-km Radius Circle

Reglonal Productivity Factor:

Animal Grazing Factor:

Meteorology Period of Record:

Recovery: 86\%

SITE SPECIFIC DATA - WATERBORNE PATHWAYS VIa PACIFIC OCEAN
Average Dilution Flow
from $\mathrm{Plant:} 640 \mathrm{ft} / \mathrm{s}$
Fish:
Edible Harvest: $2.9 \mathrm{E}^{(\mathrm{a})}$
Dilution Factor:
Invertebrates:
Edible Harvest: $2.9 \mathrm{E}^{(\mathrm{a})} \mathrm{kg} / \mathrm{yr}$
Dilution Factor: 1

(a) Seafood caught in undliuted effluent (FES, 1973). 
POPULATION DOSE-COMMITMENT ESTIMATES AND AVERAGE INDIVIDUAL DOSE-COMMITMENT HISTOGRAM FOR

\section{SN ONOFRE}

Dose Commitments (person-rem) from Liquid Pathways

Iotal Body $G I-L L I$ Ihyrold Bone Liver

Infant

$0.0 \mathrm{E}+00$

$0.0 \mathrm{E}+00$

$0.0 E+00$

$0.0 \mathrm{E}+00$

$0.0 \mathrm{E}+00$

Child

Teen

8.5E-02

$1.9 E-01$

$5.1 \mathrm{E}-03$

$1.1 \mathrm{E}-01$

3. $9 \mathrm{E}-03$

$6.6 \mathrm{E}-02$

$1.2 \mathrm{E}-01$

$5.8 E-02$

$3,3 E+00$

2.7E-02

$3.9 \mathrm{E}-01$

$1.1 \mathrm{E}-01$

4.9E-01

$3.9 \mathrm{E}+00$

3. $6 \mathrm{E}-02$

$5.7 \mathrm{E}-01$

$6.2 \mathrm{E}-01$

TOTAL.

Dose Commitments (person-rem) from Airborne Pathways

IotalBody Gl-LLl Thyrold Bone Llver Lung

$\begin{array}{lllllll}\text { Infant } & 4.1 \mathrm{E}-04 & 4.1 \mathrm{E}-04 & 5.5 \mathrm{E}-04 & 2.7 \mathrm{E}-04 & 4.1 \mathrm{E}-04 & 4.3 \mathrm{E}-04 \\ \text { ChIld } & 5.0 \mathrm{E}-03 & 5.0 \mathrm{E}-03 & 5.8 \mathrm{E}-03 & 3.0 \mathrm{E}-03 & 5.0 \mathrm{E}-03 & 5.4 \mathrm{E}-03 \\ \text { Teen } & 3.6 \mathrm{E}-03 & 3.6 \mathrm{E}-03 & 3.9 \mathrm{E}-03 & 2.2 \mathrm{E}-03 & 3.6 \mathrm{E}-03 & 4.1 \mathrm{E}-03 \\ \text { Adult } & 2.1 \mathrm{E}-02 & 2.1 \mathrm{E}-02 & 2.2 \mathrm{E}-02 & 1.3 \mathrm{E}-02 & 2.1 \mathrm{E}-02 & 2.3 \mathrm{E}-02 \\ \text { TOTAL } & 3.1 \mathrm{E}-02 & 3.1 \mathrm{E}-02 & 3.3 \mathrm{E}-02 & 1.9 \mathrm{E}-02 & 3.1 \mathrm{E}-02 & 3.3 \mathrm{E}-02\end{array}$

Production/Consumption factors:

Produce: $<1 \quad$ Milk: $<1 \quad$ Meat: $<1$

FRACTION OF POPULATION RECEIVING AN INDICATED AVERAGE TOTAL-BODY DOSE COMMITMENT FROM AIRBORNE PATHWAYS

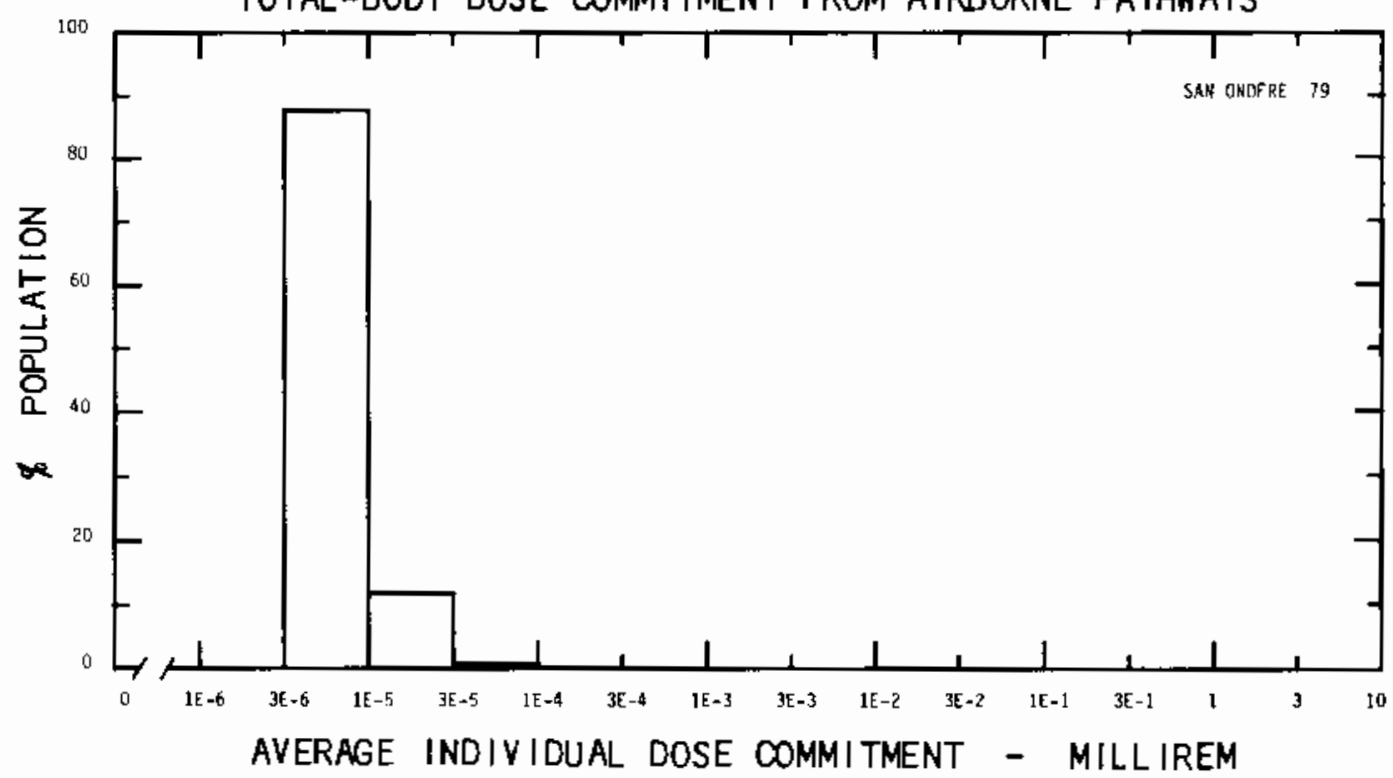


SIte: SURRY

Location: N $37.1664^{\circ}$
SURRY COUNTY, YIRGINIA

W $76.6972^{\circ}$

POPULATION DATA

Total Population Within 20-to-80-km Region: 1.8E6

Major Metropolitan Centers Within Region:

Center

Newport News SIMSA

Norfolk SMSA

Richnond SMSA
Population

370,000

820,000

600,000
Location

$16 \mathrm{~km}$ ESE

$50 \mathrm{~km} \quad \mathrm{SE}$

$78 \mathrm{~km}$

SITE SPECIFIC DATA - AIRBORNE PATHWAYS

Average Annual State Production

of Crops and Animal Products

In 80-km Radius Circle

Regional Productivity Factor:

Animal Grazing Factor:
Veg: $3.5 E 7$ kilogram

MIIk: 1.5E8 I iter

Meat: $7.4 E 7$ kilogram

0.8

0.7

Meteorology Perlod of Record: 3 MR 74 - 2 MR 75 Recovery: 91\%

Average River Flow
at Site: $25,000 \mathrm{ft}^{3} / \mathrm{s}$ (a)

Fish:

Edible Harvest: $6.055 \mathrm{~kg} / \mathrm{yr}$ Dilution Factor: 1

Invertebrates: ${ }^{(b)}$
Edible Harvest: 1.1 E6 $\mathrm{kg} / \mathrm{yr}$ Dilution Factor: 1

(a) Flow Includes fresh water river flow and sal Ine "mIxing flow" of estuary (FES, 1972).

(b) Environment primarlily salt water so invertebrates considered in Ileu of drinking water. 


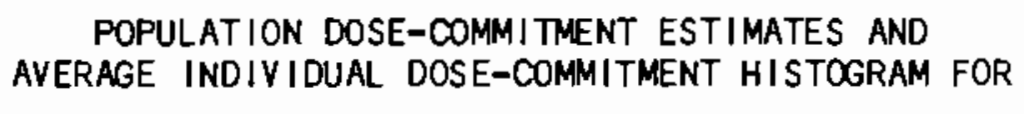

SURRY 1 AND 2

Dose Commitments (person-rem) from Liquid Pathways

Iotal Body Gl-Lلl Thyrold Bone Liver

$\begin{array}{llllll}\text { Infant } & 0.0 \mathrm{E}+00 & 0.0 \mathrm{E}+00 & 0.0 \mathrm{E}+00 & 0.0 \mathrm{E}+00 & 0.0 \mathrm{E}+00 \\ \text { Child } & 1.3 \mathrm{E}-01 & 2.3 \mathrm{E}-01 & 3.5 \mathrm{E}-01 & 6.2 \mathrm{E}-02 & 1.1 \mathrm{E}-01 \\ \text { Teen } & 9.9 \mathrm{E}-02 & 4.8 \mathrm{E}-01 & 2.4 \mathrm{E}-02 & 3.7 \mathrm{E}-02 & 9.0 \mathrm{E}-02 \\ \text { Adult } & 7.0 \mathrm{E}-01 & 4.3 \mathrm{E}+00 & 1.6 \mathrm{E}-01 & 2.1 \mathrm{E}-01 & 5.5 \mathrm{E}-01 \\ \text { TOTAL } & 9.2 \mathrm{E}-01 & 5.0 \mathrm{E}+00 & 2.2 \mathrm{E}-01 & 3.1 \mathrm{E}-01 & 7.4 \mathrm{E}-01\end{array}$

Dose Commltments (person-rem) from Alrborne Pathways

IotalBody Gl-LلL Thyrold Bone Liver Lung

$\begin{array}{lllllll}\text { Infant } & 1.7 \mathrm{E}-03 & 1.7 \mathrm{E}-03 & 5.7 \mathrm{E}-03 & 1.1 \mathrm{E}-03 & 1.7 \mathrm{E}-03 & 1.8 \mathrm{E}-03 \\ \text { ChIld } & 2.1 \mathrm{E}-02 & 2.1 \mathrm{E}-02 & 4.6 \mathrm{E}-02 & 1.2 \mathrm{E}-02 & 2.1 \mathrm{E}-02 & 2.3 \mathrm{E}-02 \\ \text { Teen } & 1.5 \mathrm{E}-02 & 1.5 \mathrm{E}-02 & 2.5 \mathrm{E}-02 & 8.8 \mathrm{E}-03 & 1.5 \mathrm{E}-02 & 1.7 \mathrm{E}-02 \\ \text { Adult } & 8.7 \mathrm{E}-02 & 8.7 \mathrm{E}-02 & 1.3 \mathrm{E}-01 & 5.3 \mathrm{E}-02 & 8.7 \mathrm{E}-02 & 9.4 \mathrm{E}-02 \\ \text { TOTAL } & 1.2 \mathrm{E}-01 & 1.2 \mathrm{E}-01 & 2.0 \mathrm{E}-01 & 7.5 \mathrm{E}-02 & 1.3 \mathrm{E}-01 & 1.3 \mathrm{E}-01\end{array}$

Production/Consumption factors:

Produce: $<1 \quad$ Milk: $<1 \quad$ Meat: $<1$

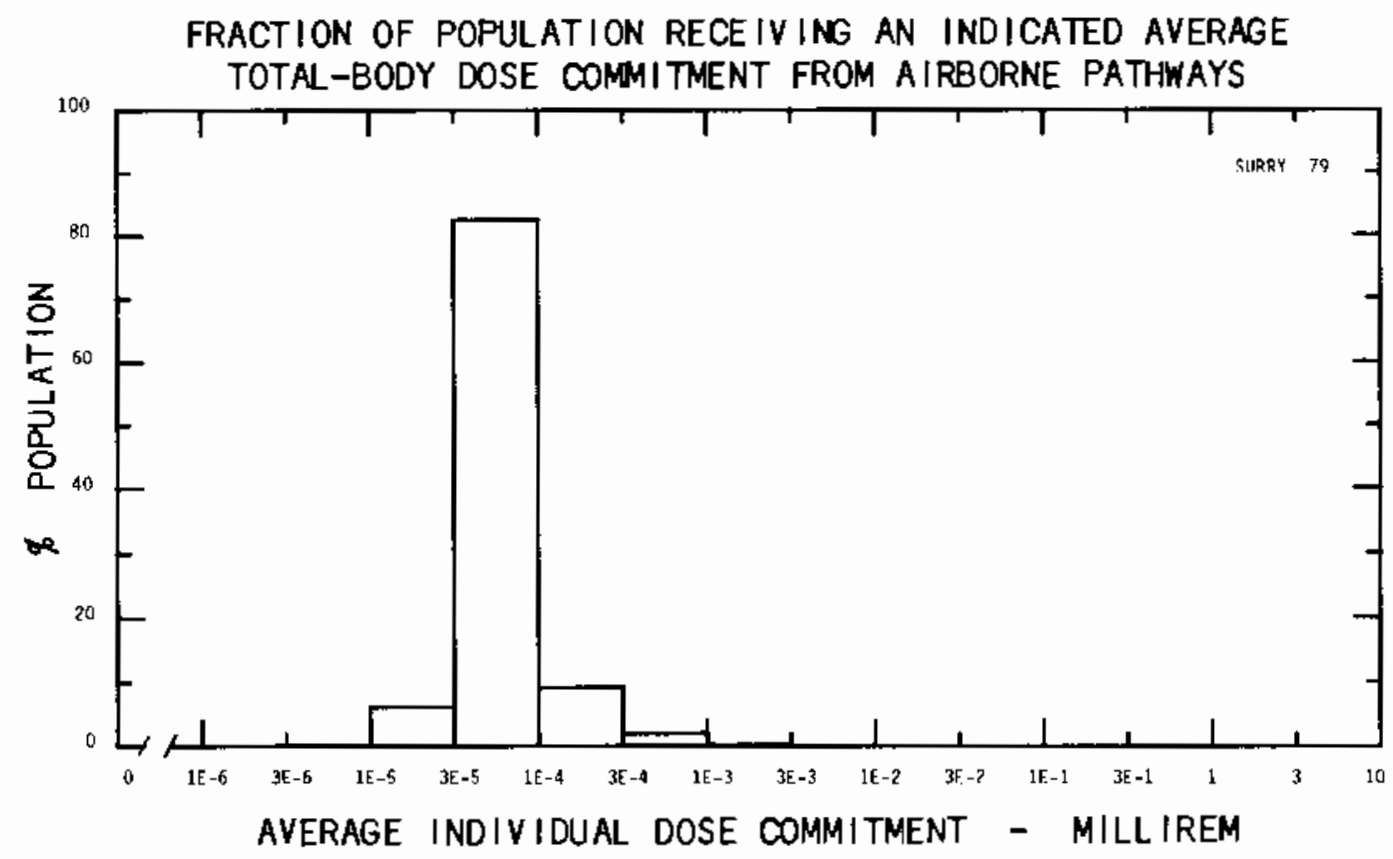


Site: THREE MILE ISLAN

Lecation: N $40.1533^{\circ}$
THREE MILE ISLND, PENISY VANIA

W6.7269

POPULATION DATA

Total Population Within 20-to-80-km Region: 1.8E6

Major Metropol itan Centers within Region:

\begin{tabular}{lccc}
\multicolumn{1}{c}{ Center } & Population & \multicolumn{2}{c}{ Lecation } \\
Lancaster SHSA & 320,000 & $40 \mathrm{~km}$ & ESE \\
Harrisburg SMSA & 410,000 & $16 \mathrm{~km}$ & $\mathrm{~N}$ \\
Reading SMSA & 290,000 & $71 \mathrm{~km}$ & ENE \\
York SiLSA & 330,000 & $23 \mathrm{~km}$ & S
\end{tabular}

SITE SPECIFIC DATA - AIRBORNE PATHWAYS

Average Annual State Production

of Crops and Anjmal Products

In 80-km Radius Circle

Reglonal Productivity Factor:

Animal Grazing Factor:

Meteorology Perlod of Record: 1 OCT 72 - 30 SEP 73 Recovery: 80 ,
Veg: $5.3 E 7$ kilogram

Milk: 5.3E8 liter

Meat: 5.4E7 kilogram

1

0.5

SITE SPECIFIC DATA - WATERBORNE PATHWAYS VIa SUSQUEHANNA RIVER

Average Rlver $\mathrm{Flow}_{3}$
at Site: $34,000 \mathrm{ft}^{3} / \mathrm{s}$

Drinking water:

Exposed Population: 200,000

Dilution Factor: 1

Fish:
Edible Harvest: (a) $\mathrm{kg} / \mathrm{y5}$
Dilution Factor: $0.025(\mathrm{~b})$

(a) No fish catch data given in FES, so generlc consumption rates used (Table A-1).

(b) Ten percent of population consumes 258 of their fish from river (FES, 1972). 


\section{POPULATION DOSE-COMMITMENT ESTIMATES AND AVERAGE INDIVIDUAL DOSE-COMMITMENT HISTOGRAM FOR \\ THREE MILE ISLND 1 ND 2}

Dose Commitments (person-rem) from Liquid Pathways

Iotal Body

Gl-LLل

Thyroid

Bone

Luer

Intant

$1.7 \mathrm{E}-03$

8.4E-04

$7.9 \mathrm{E}-02$

$7.8 \mathrm{E}-03$

$2.2 \mathrm{E}-03$

Child

3.1E-02

$1.1 \mathrm{E}-02$

$5.6 \mathrm{E}-01$

$1.4 \mathrm{E}-01$

$6.5 \mathrm{E}-02$

Teen

Adult

2. 4E-02

$6.5 \mathrm{E}-03$

5.5E-02

4. 5E-02

2.3E-01

1. $2 \mathrm{E}+00$

3. $6 \mathrm{E}-01$

2.7E-01

TOTAL

2.9E-01

$7.3 \mathrm{E}-02$

$2.1 \mathrm{E}+00$

$5.6 \mathrm{E}-01$

3.9E-01

Dose Commitments (person-rem) from Alrborne Pathways

Iotal_Body Gl-LLل Ihyrold Bone Llyer Lung

Infant

Child

$\begin{array}{ll}1.9 E+01 & 1.9 E+01 \\ 2.2 E+02 & 2.2 E+02 \\ 1.6 E+02 & 1.6 E+02 \\ 9.5 E+02 & 9.5 E+02 \\ 1.3 E+03 & 1.3 E+03\end{array}$

$3.4 \mathrm{E}+01$

1.9E+01

$3.1 \mathrm{E}+02$

$2,2 \mathrm{E}+02$

$1.9 E+01$

2.1E+01

Teen

Adult

$1.3 E+03$

$2.0 \mathrm{E}+02$

$1.6 \mathrm{E}+02$

2. $2 \mathrm{E}+02$

$2.3 \mathrm{E}+02$

TOTAL

$1.3 \mathrm{E}+03$

$9.5 \mathrm{E}+02$

$1.6 \mathrm{E}+02$

$9.9 \mathrm{E}+02$

1. $8 \mathrm{E}+02$

$1.1 E+03$

$1.3 E+03$

$1.3 E+03$

$1.0 \mathrm{E}+03$

$1.7 \mathrm{E}+03$

$1.5 E+03$

Production/Consumption factors:
Produce: <1
Milk: 2.2
Meat: <1

FRACTION OF POPULATION RECEIVING AN INDICATED AVERAGE TOTAL-BODY DOSE COMMITMENT FROM AIRBORNE PATHWAYS

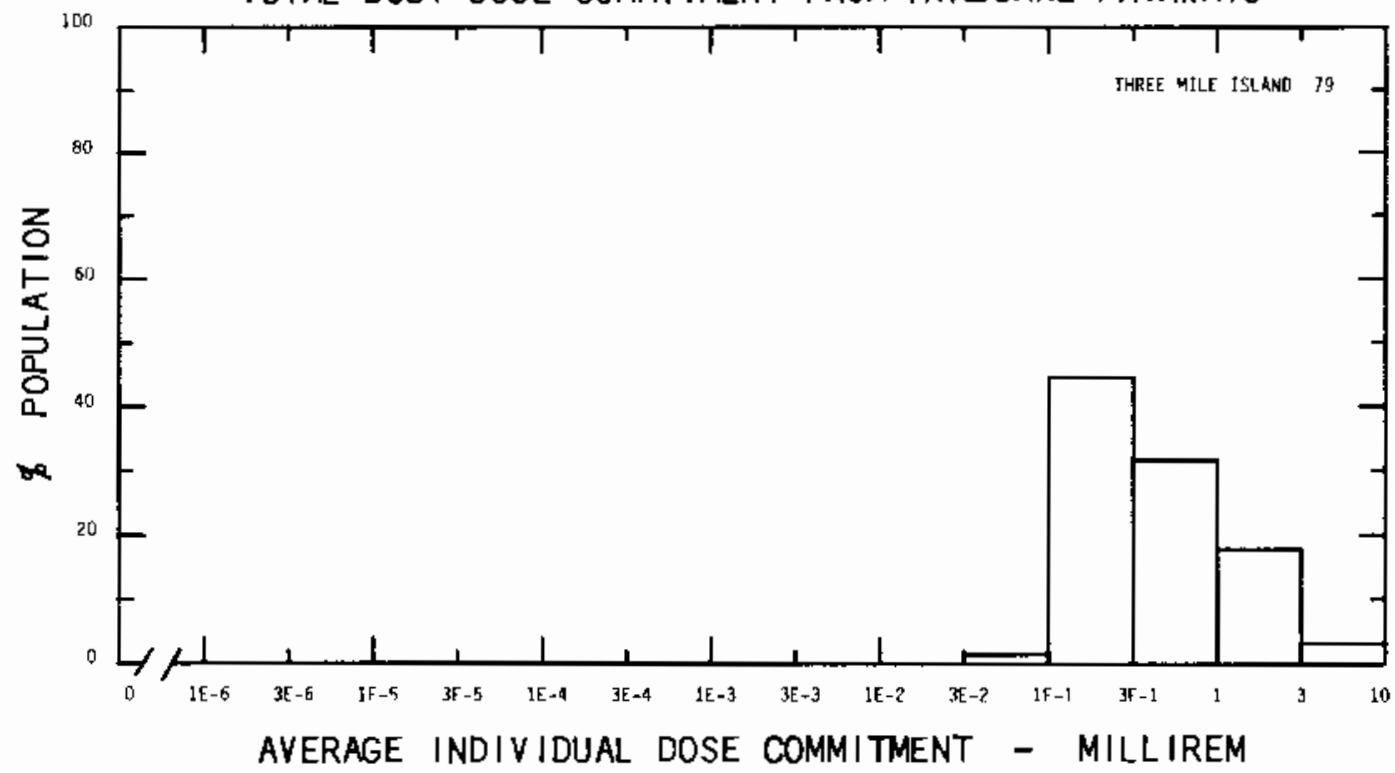


SIte: TROJAN

Location: $\quad \mathrm{N} 46.0372^{\circ}$
PRESCOT, OREGON

W $122.8847^{\circ}$

POPULATION DATA

Total Population with In 20-to-80-km Region: 1.4E6

Major Metropolitan Centers Within Region:

Center

Portl and SUSA

Longvle:

Astoria
Population

$1,200,000$

34,000

12,000
Lecation

$63 \mathrm{kn}$ SSE

$14 \mathrm{~km}$

$72 \mathrm{~km}$
SSE

Why

SITE SPECIFIC DATA - AIRBORNE PATHWAYS

Average Annual State Production

of Crops and AnImal Products

In 80-km Radius Circle

Reglonal Productivity Factor:

AnImal GrazIng Factor:

Meteorology Perlod of Record: 1 SEP 71 - 31 AUG 74 Recovery: 90\%
Veg: 6.4E7 kilogram
Mlik: 3.7E7 liter
Meat: 2.6E7 kilogram

0.9

0.75

SITE SPECIFIC DATA - WATERBORNE PATHWAYS vIa COLUMBIA RIVER

$\begin{array}{ll} & \begin{array}{l}\text { Average River Flow } \\ \text { at Slte: } 2.3 \mathrm{ES}+\mathrm{ft} / \mathrm{s}\end{array} \\ \text { Drinking Water: } & \begin{array}{l}\text { Exposed Population: } 520(\mathrm{a}) \\ \text { Dilution Factor: } 1\end{array} \\ & \text { Edible Harvest: } 1.0 \mathrm{Eg} \mathrm{kg} / \mathrm{yr} \\ \text { Fish: } & \text { Dilution Factor: } 1\end{array}$

(a) Population of Rainier divided by 4 , since residents only there for $25 \%$ of the year. 
POPULATION DOSE-COMMITMENT ESTIMATES AND

AVERAGE INDIVIDUAL DOSE-COMMITMENT HISTOGRAM FOR

\section{TROJAN}

Dose Commitments (person-rem) from Liquid Pathways

Iotal Body Ihyrold Bone LIVer

$\begin{array}{llllll}\text { Infant } & 1.8 \mathrm{E}-07 & 1.9 \mathrm{E}-07 & 1.1 \mathrm{E}-06 & 8.0 \mathrm{E}-08 & 1.8 \mathrm{E}-07 \\ \text { ChIld } & 3.2 \mathrm{E}-04 & 2.7 \mathrm{E}-03 & 1.7 \mathrm{E}-04 & 1.3 \mathrm{E}-03 & 1.5 \mathrm{E}-03 \\ \text { Teen } & 5.2 \mathrm{E}-04 & 5.7 \mathrm{E}-03 & 1.2 \mathrm{E}-04 & 7.9 \mathrm{E}-04 & 1.3 \mathrm{E}-03 \\ \text { Adult } & 5.4 \mathrm{E}-03 & 4.9 \mathrm{E}-02 & 8.1 \mathrm{E}-04 & 4.6 \mathrm{E}-03 & 7.6 \mathrm{E}-03 \\ \text { TOTAL } & 6.2 \mathrm{E}-03 & 5.7 \mathrm{E}-02 & 1.1 \mathrm{E}-03 & 6.7 \mathrm{E}-03 & 1.0 \mathrm{E}-02\end{array}$

Dose Corm I tments (person-rem) from Airborne Pathways

IotalBody Glelll Ihyrold Bone Lyer Lung

$\begin{array}{lllllll}\text { Infant } & 5.8 \mathrm{E}-04 & 5.8 \mathrm{E}-04 & 3.8 \mathrm{E}-03 & 5.6 \mathrm{E}-04 & 5.9 \mathrm{E}-04 & 6.8 \mathrm{E}-04 \\ \text { ChIld } & 6.9 \mathrm{E}-03 & 7.0 \mathrm{E}-03 & 3.9 \mathrm{E}-02 & 6.6 \mathrm{E}-03 & 7.1 \mathrm{E}-03 & 8.2 \mathrm{E}-03 \\ \text { Teen } & 5.0 \mathrm{E}-03 & 5.3 \mathrm{E}-03 & 2.0 \mathrm{E}-03 & 4.7 \mathrm{E}-03 & 5.1 \mathrm{E}-03 & 6.4 \mathrm{E}-03 \\ \text { Aduit } & 3.0 \mathrm{E}-02 & 3.2 \mathrm{E}-02 & 9.4 \mathrm{E}-02 & 2.8 \mathrm{E}-02 & 3.0 \mathrm{E}-02 & 3.5 \mathrm{E}-02 \\ \text { TOTAL } & 4.2 \mathrm{E}-02 & 4.4 \mathrm{E}-02 & 1.6 \mathrm{E}-01 & 3.9 \mathrm{E}-02 & 4.3 \mathrm{E}-02 & 5.0 \mathrm{E}-02\end{array}$

Production/Consumption factors:

Produce: $<1 \quad$ Milk: $<1 \quad$ Meat: $<1$

FRACTION OF POPULATION RECEIVING AN INDICATED AVERAGE

TOTAL-BODY DOSE COMMITMENT FROM AIRBORNE PATHWAYS

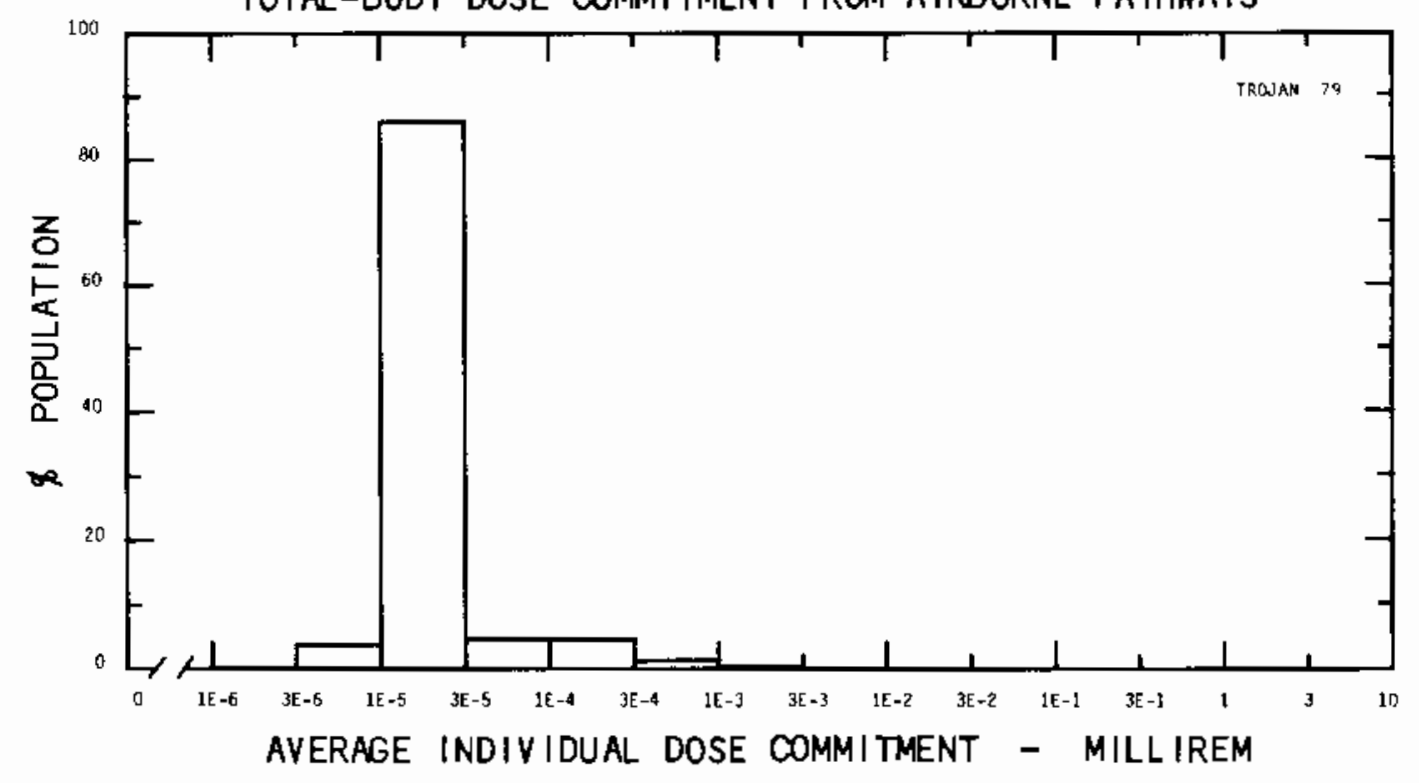


Slte: TURKEY POINT

Lecation: N $25.4336^{\circ}$
DADE COUNTY, FLORIDA

w $00.3317^{\circ}$

POPULATION DATA

Total Population Within 20-to-80-km Reglon: 2.2E6

Major Metropolitan Centers WithIn Region:

Center

MIani SIMSA
Population

$1,700,000$
Lecation

$48 \mathrm{~km}$ NNE

SITE SPECIFIC DATA - AIRBORNE PATHWAYS

Average Annual State Production

Of Crops and Animal Products

In 80-km Radius Circle

Regional Productivity Factor:

Animal Grazing Factor:

Meteorology Period of Record: 1 JAN 73 - 31 DEC 73 Recovery: 98\%

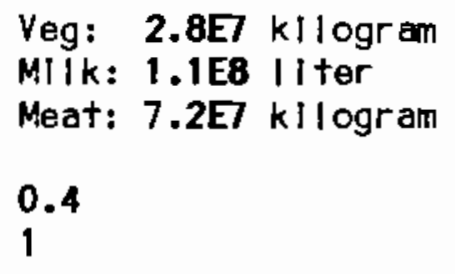

Milk: $1.0 \mathrm{E}$.

MIlk: $1.1 \mathrm{~EB}$ Ilter

Meat: $7.2 \mathrm{E} 7 \mathrm{kllogram}$

0.4

SITE SPECIFIC DATA - WATERBORNE PATHWAYS VIa BISCAYNE BAY

$\begin{array}{ll} & \begin{array}{l}\text { Average Dilution Flow } \\ \text { from Plant: } 2,400 \mathrm{ft} / \mathrm{s}\end{array} \\ & \\ & \text { Edible Harvest: (a) } \\ & \text { Dilution Factor: } 0.001 \\ & \text { Edible Harvest: }(\mathrm{a}) \\ \text { Invertebrates: } & \text { Dilution Factor: } 0.002\end{array}$

(a) No catch data given In DES (1972), so generlc consumption rates used (Table $A-1$ ). 
POPULATION DOSE-COMMITMENT ESTIMATES AND AVERAGE INDIVIDUAL DOSE-COMMITMENT HISTOGRAM FOR

TURKEY POINT 3 ND 4

Dose Commitments (person-rem) from Liquid Pathways

Iotal Body Gl-LLl Thyrold Bone Liver

Infant

Child

Teen

Adult

TOTAL

$\begin{array}{lllll}0.0 \mathrm{E}+00 & 0.0 \mathrm{E}+00 & 0.0 \mathrm{E}+00 & 0.0 \mathrm{E}+00 & 0.0 \mathrm{E}+00 \\ 4.0 \mathrm{E}-04 & 7.4 \mathrm{E}-04 & 6.3 \mathrm{E}-04 & 4.8 \mathrm{E}-04 & 6.1 \mathrm{E}-04 \\ 3.9 \mathrm{E}-04 & 1.5 \mathrm{E}-03 & 4.6 \mathrm{E}-04 & 3.0 \mathrm{E}-04 & 5.3 \mathrm{E}-04 \\ 3.2 \mathrm{E}-03 & 1.3 \mathrm{E}-02 & 3.1 \mathrm{E}-03 & 1.8 \mathrm{E}-03 & 3.3 \mathrm{E}-03 \\ 4.0 \mathrm{E}-03 & 1.5 \mathrm{E}-02 & 4.2 \mathrm{E}-03 & 2.6 \mathrm{E}-03 & 4.4 \mathrm{E}-03\end{array}$

Dose Commltments (person-rem) from Alrborne Pathways

Total_Body GL-LLI Ihyrold Bone Liver Lung

Infant

$2.4 \mathrm{E}-03$

$2.4 \mathrm{E}-03$

$1.8 \mathrm{E}-02$

$2.4 \mathrm{E}-03$

2.5E-03

2.7E-03

Chlid

2.7E-02

$2.7 \mathrm{E}-02$

$1.1 \mathrm{E}-01$

2.7E-02

2.7E-02

3. $1 \mathrm{E}-02$

$1.9 \mathrm{E}-02$

2. $0 \mathrm{E}-02$

5.4E-02

1. $9 \mathrm{E}-02$

2.0E-02

2. $4 \mathrm{E}-02$

Adult

1.2E-01

$1.2 \mathrm{E}-01$

2.3E-01

1. 2E-01

1.2E-01

1. 3E-01

TOTAL

1.7E-0 1

1.7E-01

4. $2 \mathrm{E}-01$

$1.7 \mathrm{E}-01$

$1.7 \mathrm{E}-01$

1.9E-01

Production/Consumption factors:

Produce: $<1 \quad$ Milk: $<1 \quad$ Meat: $<1$

FRACTION OF POPULATION RECEIVING AN INDICATED AVERAGE TOTAL-BODY DOSE OOMMITMENT FROM AIRBORNE PATHWAYS

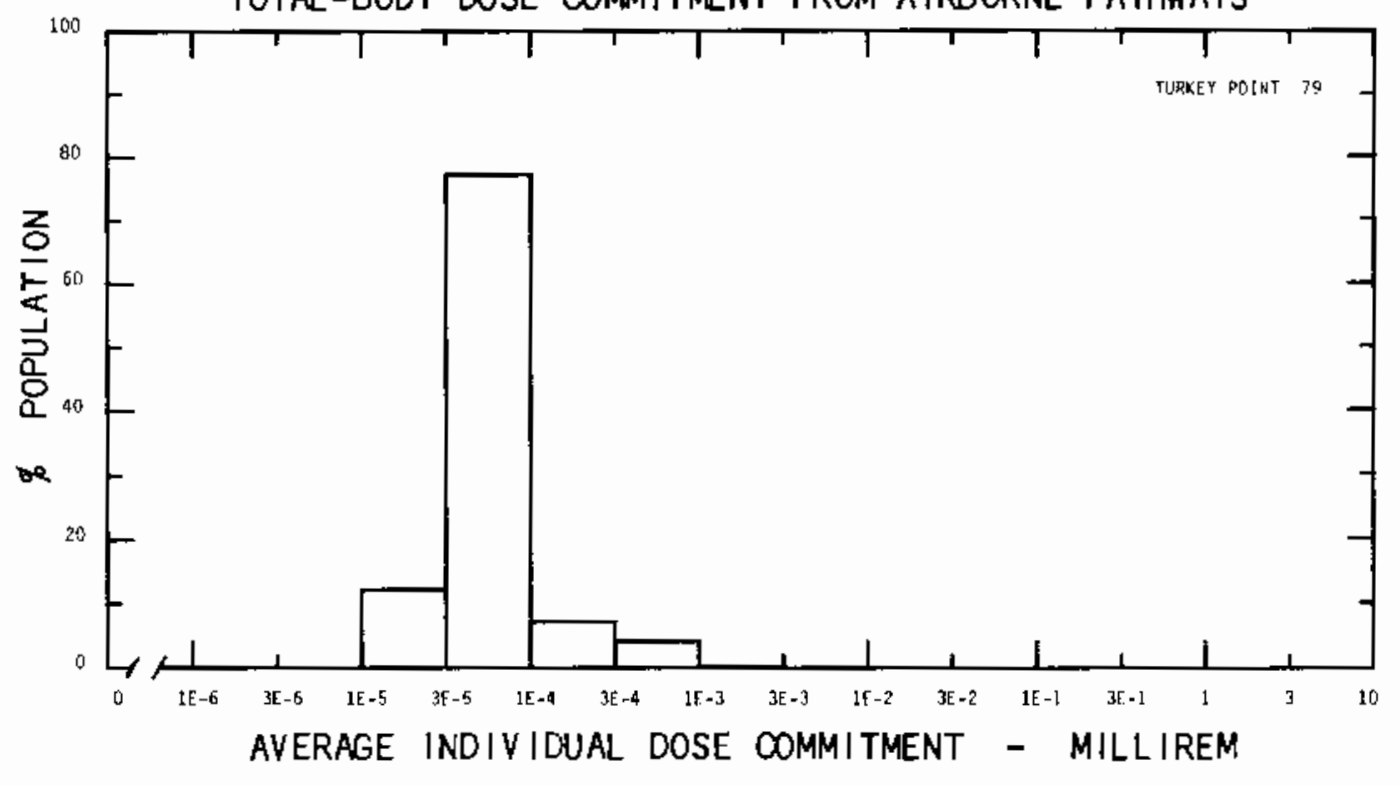


Slte: VERMONT YAKKEE

VERMON, VERAMNT

Location: $\quad N 42.7803^{\circ} \quad$ W $72.5158^{\circ}$

POPULATION DATA

Total Population within 20-to-80-km Region: 1.4E6

Major Metropolitan Centers Within Region:

center

Population

Lecation

Pittsfield

Springf leld SMSA

62,000

640,000

Norcester SUSA

700,000

$\begin{array}{lll}70 & \mathrm{~km} & \text { SH } \\ 72 & \mathrm{~km} & \mathrm{~S} \\ 80 & \mathrm{~km} & \text { SE }\end{array}$

SITE SPECIFIC DATA - AIRBORNE PATHWAYS

Average Annual State Production

of Crops and Animal Products

In 80-km Radlus Circle

Reglonal Productivity Factor:

Animal Grazing Factor:

Meteorology Perlod of Record: 1 APR 75 - 31 MRR 76
Veg: 4.4E6 kilogram

MIIk: $7.3 \mathrm{E8}$ liter

Meat: 2.7E7 kilogram

1

0.4

SITE SPECIFIC DATA - WATERBORNE PATHWAYS VIa CONNECTICUT RIVER AT VERNON POND

Average River Flow

at Site: $10,000 \mathrm{ft}^{3} / \mathrm{s}$

Drinking Water:

Exposed Population: None

FIsh:

Edible Harvest: (a)

Dilution Factor: $0.5^{(a)}$

(a) No fish catch data given in FES (1972), thus $1 / 2$ population assumed to eat $f$ ish at level of generic consumption rates (Table A-1). 


\section{POPULATION DOSE-COMMITMENT ESTIMATES AND AVERAGE INDIVIDUAL DOSE-COMMITMENT HISTOGRAM FOR \\ VERAONT YANEE}

Dose Commitments (person-rem) from Liquid Pathways

Iotal Body Gi-LLI Thyrodd Bone Liver

$\begin{array}{llllll}\text { Infant } & 0.0 \mathrm{E}+00 & 0.0 \mathrm{E}+00 & 0.0 \mathrm{E}+00 & 0.0 \mathrm{E}+00 & 0.0 \mathrm{E}+00 \\ \text { Child } & 1.3 \mathrm{E}-04 & 1.7 \mathrm{E}-04 & 2.9 \mathrm{E}-05 & 3.7 \mathrm{E}-04 & 4.5 \mathrm{E}-04 \\ \text { Teen } & 1.8 \mathrm{E}-04 & 3.4 \mathrm{E}-04 & 2.5 \mathrm{E}-05 & 2.4 \mathrm{E}-04 & 4.0 \mathrm{E}-04 \\ \text { Adult } & 1.8 \mathrm{E}-03 & 3.0 \mathrm{E}-03 & 1.9 \mathrm{E}-04 & 1.4 \mathrm{E}-03 & 2.4 \mathrm{E}-03 \\ \text { TOTAL } & 2.1 \mathrm{E}-03 & 3.5 \mathrm{E}-03 & 2.4 \mathrm{E}-04 & 2.0 \mathrm{E}-03 & 3.3 \mathrm{E}-03\end{array}$

Dose Commitments (person-rem) from Alrborne Pathways Iotal Body Gl-Lلl Ihyrold Bone Liver Lung

$\begin{array}{lllllll}\text { Infant } & 6.8 \mathrm{E}-03 & 6.4 \mathrm{E}-03 & 2.5 \mathrm{E}-01 & 7.4 \mathrm{E}-03 & 8.2 \mathrm{E}-03 & 7.4 \mathrm{E}-03 \\ \text { Child } & 7.5 \mathrm{E}-02 & 7.1 \mathrm{E}-02 & 1.7 \mathrm{E}+00 & 7.8 \mathrm{E}-02 & 8.3 \mathrm{E}-02 & 8.7 \mathrm{E}-02 \\ \text { Teen } & 5.4 \mathrm{E}-02 & 5.2 \mathrm{E}-02 & 8.6 \mathrm{E}-01 & 5.3 \mathrm{E}-02 & 5.8 \mathrm{E}-02 & 6.8 \mathrm{E}-02 \\ \text { Adult } & 3.2 \mathrm{E}-01 & 3.1 \mathrm{E}-01 & 3.6 \mathrm{E}+00 & 3.1 \mathrm{E}-01 & 3.3 \mathrm{E}-01 & 3.7 \mathrm{E}-01 \\ \text { TOTAL } & 4.6 \mathrm{E}-01 & 4.4 \mathrm{E}-01 & 6.4 \mathrm{E}+00 & 4.5 \mathrm{E}-01 & 4.8 \mathrm{E}-01 & 5.3 \mathrm{E}-01\end{array}$

Production/Consumption factors:

Produce: $<1 \quad$ Milk: $3.9 \quad$ Meat: <1

FRACTION OF POPULATION RECEIVING AN INDICATED AVERAGE TOTAL-BODY DOSE COMMITMENT FROM AIRBORNE PATHWAYS

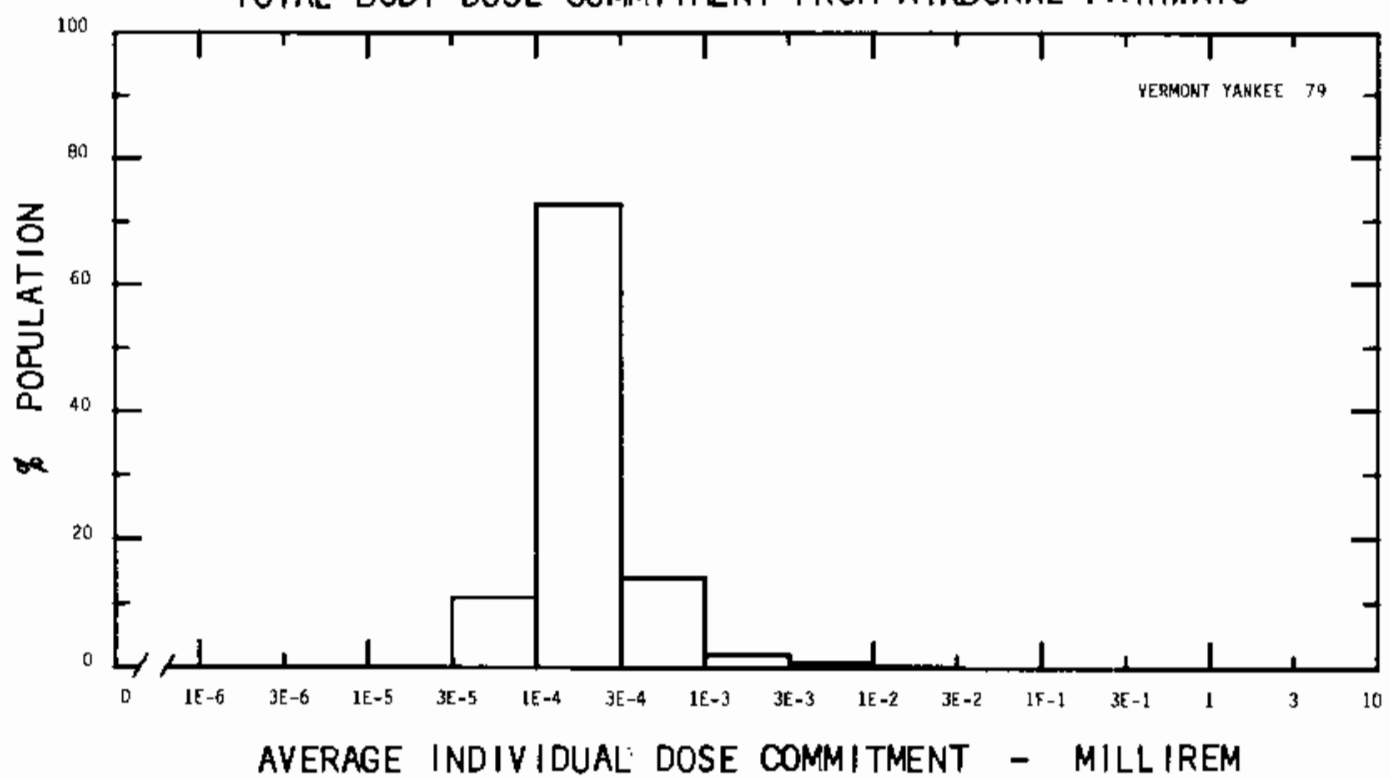


SIte: YANKEE RONE

Location:
RONE, MASSACHUSETTS

W $72.9247^{\circ}$

POPULATION DATA

Total Population within 20-to-80-km Region: 1.6E6

Major Metropolitan Centers Within Reglon:

Center

Springfleld SMSA

PIttsfield

Albany SMSA
Population

590,000

58,000

790,000
Location

$64 \mathrm{~km} \quad$ SSE

$34 \mathrm{~km} \quad \mathrm{SW}$

$72 \mathrm{~km} \mathrm{~W}$

SITE SPECIFIC DATA - AIRBORNE PATHWAYS

Average Annual State Production

Of Crops and Anlmal Products

in 80-km Radius Circle

Regional Productivity Factor:

AnImal Grazing Factor:

Meteorology Period of Record: 1 OCT 71 - 30 SEP 72 Recovery: 94\%
Veg: 2.0E7 kilogram

Mlik: 2.6E8 liter

Meat: 1.6E7 kilogram

1

0.5

SITE SPECIFIC DATA - WATERBORNE PATHWAYS vIa DEERFIELD RIVER

Average River Flow

at Site: $570 \mathrm{ft}^{3} / \mathrm{s}$

Drinking water:

Exposed Population: None

Fish:

Edible Harvest: (a)

Dilution Factor: $\mathbf{0 . 0 2 5}{ }^{(b)}$

(a) No catch data avallable, so generlc consumption rates used (Table A-1).

(b) Ten percent of population obtain $25 \%$ of their fish from river. 


\section{POPULATION DOSE-COMMITMENT ESTIMATES AND AVERAGE INDIVIDUAL DOSE-COMMITMENT HISTOGRAM FOR}

\section{YNAKEE RONE}

Dose Cormitments (person-rem) from L lquld Pathways

IotalBody Gl-LLI Thyrold Bone Lver

Infant

Child

Teen

Adult

TOTAL
$0.0 \mathrm{E}+00$

$1.6 \mathrm{E}-02$

$3.1 E-02$

$3.3 E-01$

$3.8 \mathrm{E}-01$
$0.0 \mathrm{E}+00$

1. $5 \mathrm{E}-03$

2.1E-03

$1.7 \mathrm{E}-02$

2. $0 \mathrm{E}-02$
$0.0 \mathrm{E}+00 \quad 0.0 \mathrm{E}+00$

$6.0 \mathrm{E}-03 \quad 7.4 \mathrm{E}-02$

$4.5 \mathrm{E}-03 \quad 4.5 \mathrm{E}-02$

3. $0 \mathrm{E}-\mathrm{D} 2 \quad 2.6 \mathrm{E}-01$

4.1E-02 3.8E-01
$0.0 \mathrm{E}+00$

8.7E-02

$7.5 \mathrm{E}-02$

4.5E-01

$6.1 \mathrm{E}-01$

Dose Commitments (person-rem) from Airborne Pathways

Iotal Body Gl-LLI Ihyrold Bene Llver Lung

$\begin{array}{lllllll}\text { Infant } & 6.6 \mathrm{E}-04 & 6.6 \mathrm{E}-04 & 8.0 \mathrm{E}-04 & 2.4 \mathrm{E}-03 & 6.6 \mathrm{E}-04 & 6.6 \mathrm{E}-04 \\ \text { Child } & 5.0 \mathrm{E}+03 & 5.0 \mathrm{E}-03 & 5.8 \mathrm{E}-03 & 1.7 \mathrm{E}-02 & 5.1 \mathrm{E}-03 & 5.2 \mathrm{E}-03 \\ \text { Teen } & 2.3 \mathrm{E}-03 & 2.3 \mathrm{E}-03 & 2.6 \mathrm{E}-03 & 5.8 \mathrm{E}-03 & 2.3 \mathrm{E}-03 & 2.4 \mathrm{E}-03 \\ \text { Adult } & 1.0 \mathrm{E}-02 & 1.0 \mathrm{E}-02 & 1.1 \mathrm{E}-02 & 2.0 \mathrm{E}-02 & 1.0 \mathrm{E}-02 & 1.1 \mathrm{E}-02 \\ \text { TOTAL } & 1.8 \mathrm{E}-02 & 1.8 \mathrm{E}-02 & 2.1 \mathrm{E}-02 & 4.6 \mathrm{E}-02 & 1.8 \mathrm{E}-02 & 1.9 \mathrm{E}-02\end{array}$

Production/Consumption factors:

Produce: <1 Milk: $1.3 \quad$ Meat: <1

FRACTION OF POPULATION RECEIVING AN INDICATED AVERAGE TOTAL-BODY DOSE COMMITMENT FROM AIRBORNE PATHWAYS

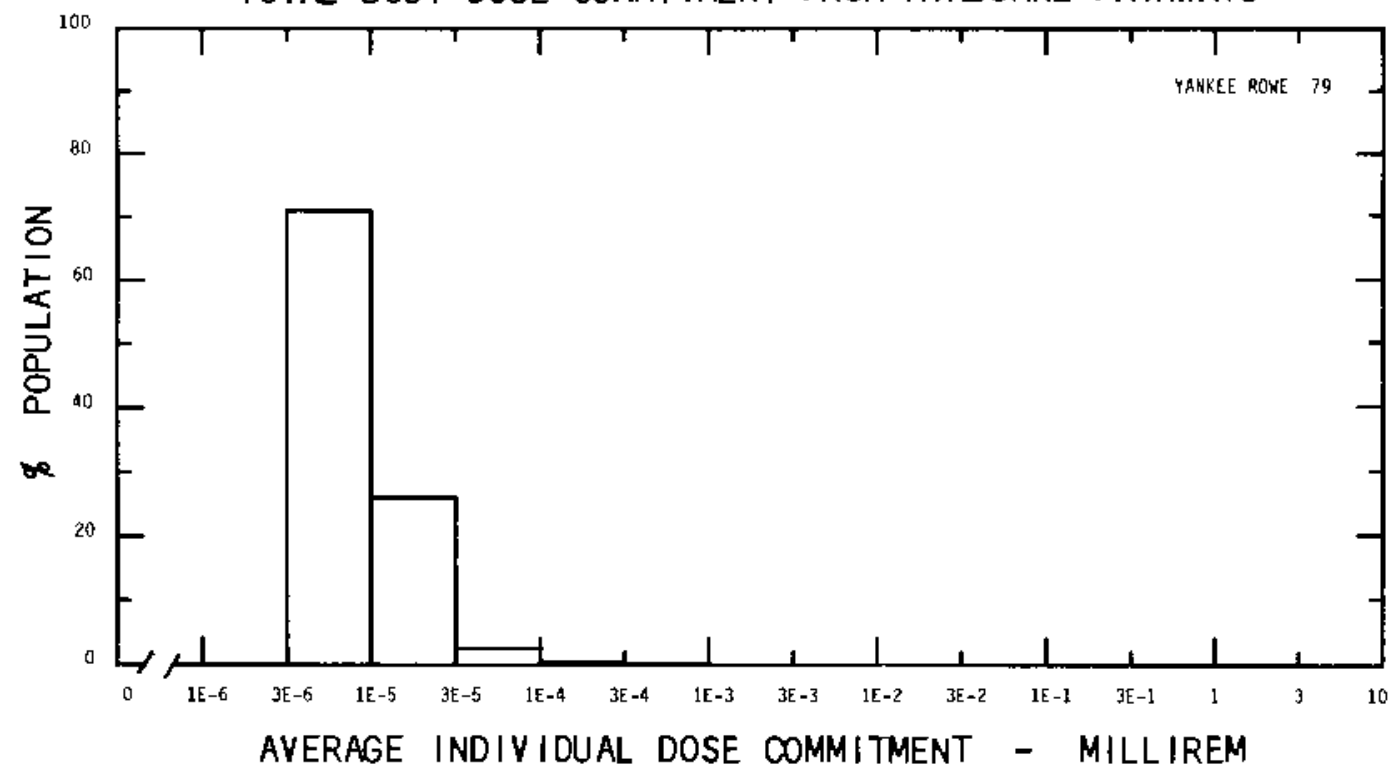


Site: ZION

Lecation:

POPULATION DATA

Total Population within 20-to-80-km Reglon: 7.0E6

Major Metropolitan Centers Within Region:

Center
ChI cago SuSA (2/3)
MIl raukee SISA
Kenosha

Center

MII raukee SISA

Kenosha
Population

$4,700,000$

$1,400,000$

80,000
Lecation

$64 \mathrm{~km} \quad$ SSE

$64 \mathrm{~km} N$

$14 \mathrm{~km}$ NM

SITE SPECIFIC DATA - AIRBORNE PATHWAYS

Average Annual State Production

of Crops and Animal Products

In 80-km Radius Circle

Reglonal Productivity Factor:

AnImal Grazing Factor:
Veg: $1.1 \mathrm{~EB} \mathrm{kilogram}$

MIlk: 1.8E8 liter

Meat: 1.9E8 kilogram

0.5

0.5

Meteorology Period of Record: 1 JAN 74 - 31 DEC 75 Recovery: 88\%
Average Dilution $\mathrm{FI}$ Qw
from Plant: $1000 \mathrm{ft}^{3} / \mathrm{s}$
Drinking Water:
Exposed Population: 6,700,000
Dilution Factor: $\mathbf{0 . 0 3 7}$
Fish:

\footnotetext{
(a) Drinking water dilution factor estimated by averaging dilution factors derlved from FES (1972) suitably welghted for population.

(b) Dilution factor derived from FES (1972).
} 


\section{POPULATION DOSE-COMMITMENT ESTIMATES AND AVERAGE INDIVIDUAL DOSE-COMMITMENT HISTOGRAM FOR \\ ZION 1 ND 2}

Dose Commitments (person-rem) from Liquld Pathways

Iotal Body

GI-WLI

Thyreald

Bone

Llver

Infant

ChIId

$1.4 \mathrm{E}-01$

1.3E-01

2.1E-01

$7.6 \mathrm{E}-02$

2.2E-01

Teen

Adult

1. $7 \mathrm{E}+00$

1. $5 \mathrm{E}+00$

2. $0 \mathrm{E}+00$

1. $3 \mathrm{E}+00$

$2.8 \mathrm{E}+00$

8.6E-01

$6.6 \mathrm{E}-01$

7.2E-01

4.8E-01

$1.3 \mathrm{E}+00$

TOTAL

8. $1 \mathrm{E}+00$

$5.7 \mathrm{E}+00$

$5.8 \mathrm{E}+00$

$2.9 \mathrm{E}+00$

9. $2 \mathrm{E}+00$

1.1E+0 1

$8.0 \mathrm{E}+00$

$8.8 \mathrm{E}+00$

4.7E+00

1. $4 \mathrm{E}+01$

Dose Commitments (person-rem) from Airborne Pathways Iotal Body Gl-LLL Thyrold Bone Liver Lung

$\begin{array}{lllllll}\text { Infant } & 5.0 \mathrm{E}-02 & 5.0 \mathrm{E}-02 & 5.3 \mathrm{E}-02 & 5.0 \mathrm{E}-02 & 5.0 \mathrm{E}-02 & 5.5 \mathrm{E}-02 \\ \text { ChIId } & 5.5 \mathrm{E}-01 & 5.5 \mathrm{E}-01 & 5.8 \mathrm{E}-01 & 5.6 \mathrm{E}-01 & 5.6 \mathrm{E}-01 & 6.3 \mathrm{E}-01 \\ \text { Teen } & 4.1 \mathrm{E}-01 & 4.1 \mathrm{E}-01 & 4.2 \mathrm{E}-01 & 4.1 \mathrm{E}-01 & 4.1 \mathrm{E}-01 & 4.9 \mathrm{E}-01 \\ \text { Aduit } & 2.5 \mathrm{E}+00 & 2.5 \mathrm{E}+00 & 2.5 \mathrm{E}+00 & 2.5 \mathrm{E}+00 & 2.5 \mathrm{E}+00 & 2.8 \mathrm{E}+00 \\ \text { TOTAL } & 3.5 \mathrm{E}+00 & 3.5 \mathrm{E}+00 & 3.6 \mathrm{E}+00 & 3.5 \mathrm{E}+00 & 3.5 \mathrm{E}+00 & 3.9 \mathrm{E}+00\end{array}$

Production/Consumption factors:

Produce: $<1 \quad$ Milk: $<1 \quad$ Meat: $<1$

FRACTION OF POPULATION RECEIVING AN INDICATED AVERAGE TOTAL-BODY DOSE COMMITMENT FROM AIRBORNE PATHWAYS

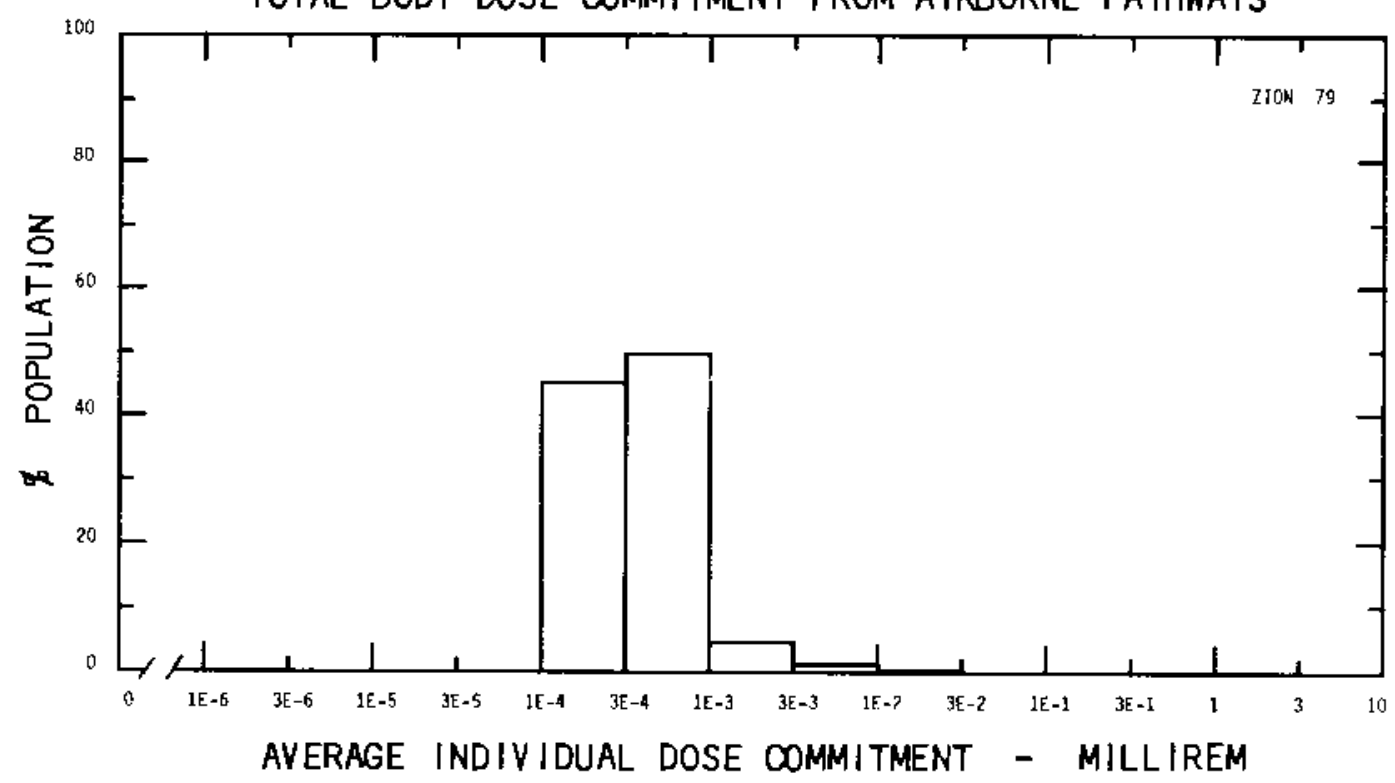





\section{REFERENCES}

Baker, D. A., J. K. Sol dat and E. C. Watson, 1977. Pepulation Dose Commitments Due to Radioact Ive Releases from Nuclear Power Plant Sites In 1975, PNL-2439. Paclflic Northwest Laboratory, RIchland, WA.

Baker, D. A. 1979. Populatien Dose Commitments Due te Radloactlve Releases from Nuclear. Power Plant Sltes in 1976, NUREG/CR-1125, PNL2940. U.S. Nuclear Regulatory Commission, Washington, D.C.*

Baker, D. A. 1980. Population Dose Commitments Due to Radioact ive Releases from Nuclear Power Plant Sites in 1977, NUREG/CR-1 498, PNL3324. U.S. Nuclear Regulatory Commission, Washington, D.C.*

Einal Environmental Statement Concerning Proposed Rule-Making Action: Numerical Guides for Deslon ob Jectives and limiting Conditions for Operation to Meet the Criterion "As Low As Practicable" for Rad loactive Material in Llaht-Water-Cooled Nuclear Power Reactor Effluents. 1973. WASH-1258, Vol. 1. Directorate of Regulatory Standards, U.S. Atom Ic Energy Commission, Washington, D.C.

Noshkin, V. E., W. L. Robison and F. L. Harrison. 1976. Radiologlcal Dose to Man Through the Mar Ine Pathway from Reactor Operations at Humboldt Bay, Callfornla, UCRL-52160. Lawrence Livermore Laboratory, Livermore, CA.

Peloquin, R. A., J. D. Schwab, and D. A. Baker. 1982. Repulation Dose Commitments Due to Rad loactive Releases from Nuclear Power Plant Sites In 1978, NUREG/CR-2201, PNL-3867. U. S. Nuclear Regulatory Commission, Washington, D. C.*

Tichler, J. and C. Benkovitz. 1981. Radieactlve Materlals Released from Nuclear Power Plants. Annual Report 1978, NJREG/CR-1497, BNL-NUREG51192. U.S. Nuclear Regulatory Commission, Washington, D.C.*

* Avallable for purchase from the NRC/GPO Sales Program, U.S. Nuclear Regulatory Commission, Washington, D.C 20555, and the National Technical Information Service, Springfield, VA 22161. 



\section{APPENDIX}

\section{MODELS AND GENERIC PARAMETERS}

The calculational models used were primarlly those given in the Nuclear Regulatory CommIssion's Regulatory Gulde 1.109 (1977). Computer programs were written to use these models to generate population dose comitments for four age groups. The percentages of the population comprising the four age groups were 1.44\%, Infant; 16.0\%, child; $11.7 \%$, teenager; and 70.9\%, adult (Population Estimates and Projections, 1975 ). Where posslble, the site-dependent parameters were taken from the environmental statements issued for each reactor (Table 3). The generic parameters used for this study such as consumption rates, occupancy factors and holdup times are glven in Table A-i and A-2 below. It should be noted that generic consumption rates for aquatic foods and inhalation rates are taken from Regulatory Gulde 1.109 (1977). Bloaccumulation factors and terrestrial food transfer factors were taken from Regulatory Gulde 1.109 (1977). Dose comm itment factors for the four age groups were taken from Hoenes and Soldat (1977).

IABLE A-1. Generic Consumption Rates and Occupancy Factors Used for the Study of Average Members of the Population (a)

$\begin{array}{lcccc}\text { Pathway } & \text { Infant } & \text { chld } & \text { Ieenager } & \text { Adult } \\ \text { Fruits, vegetables and grain } & 0 & 200 & 240 & 190 \\ \text { (kg/yr) } & & & & \\ \text { Milk (L/yr) } & 170 & 170 & 200 & 110 \\ \text { Meat and poultry (kg/yr) } & 0 & 37 & 59 & 95 \\ \text { Fish (kg/yr) (b) } & 0 & 2.2 & 5.2 & 6.9 \\ \text { Invertebrates (kg/yr) } & 0 & 0.33 & 0.75 & 1.0 \\ \text { Drinking water (L/yr) } & 170^{(\mathrm{c})} & 260 & 260 & 370 \\ \text { Inhalation (m/yr) } & 1400^{(\mathrm{d})} & 3700 & 8000 & 8000 \\ \text { Air submersion and ground } & 0.5 & 0.5 & 0.5 & 0.5 \\ \text { Irradiation occupancy } & & & & \\ \text { factor } & & & & \end{array}$

(a) Regulatory Guide 1.109 (1977)

(b) Both fresh- and sal twater

(c) Assumed to be equal to milk consumption

(d) Same as for maximum individual 
IABLEA-2. Holdup Times Between Harvest and Consumption of Foods $(a)$

Food

Fruits, grains and vegetables $M i \mid k^{(b)}$

Meat ${ }^{(b)}$

Aquatic foods ( $f$ ish and Invertebrates) DrInking Water
Holdup Iime (days)

14

4

20

7

1

(a) Regulatory Gulde 1.109 (1977)

(b) Value given is time after mllking or slaughter. For the portion of the time animals were fed stored feed, an additional 90 days was added to the holdup time.

\section{SOURCE TERMS}

The doses were estimated using the measured releases as reported by the site operators for 1979 (TIchler and Benkovitz, 1981). (a) These releases Include al I radionucl ides specifled by the NRC to be measured and reported by the operators of all commerclal nuclear power plants. Radionuclides given as a combination of parent-daughter isotopes such as $\mathrm{Y} / \mathrm{Sr}-$ 90, $\mathrm{Zr} / \mathrm{Nb}-95, \mathrm{Ba} / \mathrm{La}-140,1 / \mathrm{Xe}-133$ and $\mathrm{Pr} / \mathrm{Ce}-144$ were divided evenly between the parent and daughter.

The radionuclides used in this study, along ith their half-lives, are given in Table A-3. Note that the "+D" after some of the nuclides indicates that the decay energy of the daughter is Included with the parent. Thus, whenever a parent nuclide release is specified, the result of the dose calculation will be as though an additional equilibrium amount of the daughter nuclide is speclifled. The daughter nuclide itself will be included separately if it can be released independently of the parent and/or if it has a relatively long half-life.

\section{METEOROLOGY}

When more than one set of meteorological (jolnt frequency) data were avallable for a slte, the one which appeared to be the most rellable was used to generate atmospheric transport factors. Factors were calculated for 16 compass polnts, and ten radil from 2 to $80 \mathrm{~km}$ (see Table A-4) using the NRC computer program XOQDOQ (Sagendorf 1977).

(a) Very short-lived isotopes such as $\mathrm{Kr}-90,91,93,94, \mathrm{X}_{e-139}, 140,141$, 143 and Rb-88M; those not likely to be produced; and those which were daughters hose decay energles were accounted for in the dose factor for the parent were not included in the dose. 
IABLEA-3. Radionucl ides Considered in This Study

\begin{tabular}{|c|c|c|c|c|c|}
\hline No. & Nuclde & $\begin{array}{c}\text { Decay Constant } \\
(1 / \mathrm{sec}) \\
\end{array}$ & No. & Nucllde & $\begin{array}{c}\text { Decay Constant } \\
(1 / \mathrm{sec})\end{array}$ \\
\hline 1 & $\mathrm{H}-3$ & $1.78 \mathrm{E}-09$ & 43 & $\mathrm{Nb}-97$ & $1.57 \mathrm{E}-04$ \\
\hline 2 & $80-10$ & $1.37 E-14$ & 44 & Mo $-99+D$ & $2.92 \mathrm{E}-06$ \\
\hline 3 & $C-14$ & $3.83 E-12$ & 45 & Tc-99M & $3.19 E-05$ \\
\hline 4 & $N-13$ & $1.16 \mathrm{E}-03$ & 46 & $R u-103+D$ & $2.02 E-07$ \\
\hline 5 & $F-18$ & $1.05 E-04$ & 47 & $R u-106+D$ & $2.17 \mathrm{E}-08$ \\
\hline 6 & $\mathrm{Na}-22$ & $8.44 E-09$ & 48 & $A g-110 M+D$ & $3.19 \mathrm{E}-08$ \\
\hline 7 & $\mathrm{Na}-24$ & $1.28 E-05$ & 49 & $\mathrm{Cd}-115 \mathrm{M}$ & $1.80 E-07$ \\
\hline 8 & $\mathrm{Ar}-41$ & $1.05 E-04$ & 50 & $c d-115$ & $3.60 \mathrm{E}-06$ \\
\hline 9 & $\mathrm{Sc}-46$ & $9.58 \mathrm{E}-08$ & 51 & $S n-125+D$ & $8.31 E-07$ \\
\hline 10 & $\mathrm{Cr}-51$ & $2.89 E-07$ & 52 & $S b-124$ & $1.33 E-07$ \\
\hline 11 & $M n-54$ & $2.57 E-08$ & 53 & $S b-125+D$ & $8.06 \mathrm{E}-09$ \\
\hline 12 & $M n-56$ & $7.47 \mathrm{E}-05$ & 54 & $\mathrm{Te}-132+\mathrm{D}$ & $2.47 \mathrm{E}-06$ \\
\hline 13 & $\mathrm{Fe}-55$ & $8.14 E-09$ & 55 & $T_{\theta}-133 M+D$ & $2.09 E-04$ \\
\hline 14 & $F e-59$ & $1.80 \mathrm{E}-07$ & 56 & $1-131+D$ & $9.97 \mathrm{E}-07$ \\
\hline 15 & $\mathrm{Co}-57$ & $2.97 \mathrm{E}-08$ & 57 & $1-132$ & $8.42 \mathrm{E}-05$ \\
\hline 16 & $\mathrm{Co}-58$ & $1.12 \mathrm{E}-07$ & 58 & $1-133+D$ & $9.25 \mathrm{E}-06$ \\
\hline 17 & $\operatorname{Co}-60$ & 4.17E-09 & 59 & $1-134$ & $2.20 \mathrm{E}-04$ \\
\hline 18 & $\mathrm{~N}:-57$ & $5.35 \mathrm{E}-06$ & 60 & $1-135+D$ & $2.92 \mathrm{E}-05$ \\
\hline 19 & $\mathrm{Ni}-63$ & $2.20 \mathrm{E}-10$ & 61 & $\mathrm{Xe}-131 \mathrm{M}$ & $6.69 E-07$ \\
\hline 20 & $\mathrm{Ni}-65$ & $7.64 \mathrm{E}-05$ & 62 & $X_{e}-133 M$ & $3.61 \mathrm{E}-06$ \\
\hline 21 & Cu-64 & $1.52 \mathrm{E}-05$ & 63 & $x e-133$ & $1.52 \mathrm{E}-06$ \\
\hline 22 & $2 n-65$ & $3.31 E-08$ & 64 & $\mathrm{Xe}-135 \mathrm{M}$ & $7.56 \mathrm{E}-04$ \\
\hline 23 & $Z n-69 M+D$ & $1.39 \mathrm{E}-05$ & 65 & $x_{\theta-135}$ & $2.10 \mathrm{E}-05$ \\
\hline 24 & As-76 & $7.32 \mathrm{E}-06$ & 66 & $x e-137$ & $3.01 E-03$ \\
\hline 25 & $\mathrm{Br}-82$ & $5.44 \mathrm{E}-06$ & 67 & $x e-138+D$ & $8.14 E-04$ \\
\hline 26 & $\mathrm{Kr}-83 \mathrm{M}$ & $1.04 \mathrm{E}-04$ & 68 & $C_{s}-134$ & $1.07 E-08$ \\
\hline 27 & $\mathrm{Kr}-85 \mathrm{M}$ & $4.31 E-05$ & 69 & $C_{5}-136$ & $6.17 \mathrm{E}-07$ \\
\hline 28 & $\mathrm{Kr}-85$ & $2.05 \mathrm{E}-09$ & 70 & $C s-137+D$ & $7.31 E-10$ \\
\hline 29 & $\mathrm{Kr}-87$ & $1.52 E-04$ & 71 & $\mathrm{Cs}-138$ & $3.58 \mathrm{E}-04$ \\
\hline 30 & $\mathrm{Kr}-88+\mathrm{D}$ & $6.89 \mathrm{E}-05$ & 72 & $C s-139+D$ & $1.24 \mathrm{E}-03$ \\
\hline 31 & $\mathrm{Kr}-89$ & $3.64 \mathrm{E}-03$ & 73 & $\mathrm{Ba}-139$ & $1.39 E-04$ \\
\hline 32 & $\mathrm{Rb}-88$ & $6.53 \mathrm{E}-04$ & 74 & $\mathrm{Ba}-140+\mathrm{D}$ & $6.28 \mathrm{E}-07$ \\
\hline 33 & $\mathrm{Rb}-89+\mathrm{D}$ & $7.61 \mathrm{E}-04$ & 75 & $\mathrm{La}-140$ & $4.78 E-06$ \\
\hline 34 & $\mathrm{Sr}-89+\mathrm{D}$ & $1.59 E-07$ & 76 & $\operatorname{La}-141$ & $4.97 \mathrm{E}-05$ \\
\hline 35 & $\mathrm{Sr}-90+\mathrm{D}$ & $7.58 \mathrm{E}-10$ & 77 & $\mathrm{Ce}-141$ & $2.47 E-07$ \\
\hline 36 & $\mathrm{Sr}-91+\mathrm{D}$ & $2.03 \mathrm{E}-05$ & 78 & $\mathrm{Ce}-144+\mathrm{D}$ & $2.83 E-08$ \\
\hline 37 & $\mathrm{Sr}-92+\mathrm{D}$ & $7.11 \mathrm{E}-05$ & 79 & $E u-152$ & $1.69 \mathrm{E}-09$ \\
\hline 38 & $Y-90$ & $3.01 \mathrm{E}-06$ & 80 & Eu-154 & $2.55 E-09$ \\
\hline 39 & $Y-91 M+D$ & $2.32 E-04$ & 81 & $w-187$ & $8.06 \mathrm{E}-06$ \\
\hline 40 & $\mathrm{Zr}-95+\mathrm{D}$ & $1.22 \mathrm{E}-07$ & 82 & Th $-232+D$ & $1.57 E-18$ \\
\hline 41 & $\mathrm{Zr}-97+\mathrm{D}$ & $1.14 \mathrm{E}-05$ & 83 & $N p-239$ & $3.42 E-06$ \\
\hline 42 & Nb-95 & $2.29 E-07$ & & & \\
\hline
\end{tabular}


IABLE A-4. Radlus Intervals and MIdpoints for Alrborne Dose Calculations ( $\mathrm{km}$ )

$\begin{array}{cc}\text { Interval } & \text { Midpolnt } \\ 2-3 & 2.5 \\ 3-4 & 3.5 \\ 4-6 & 5 \\ 6-9 & 7.5 \\ 9-14 & 11.5 \\ 14-20 & 17 \\ 20-30 & 25 \\ 30-40 & 35 \\ 40-60 & 50 \\ 60-80 & 70\end{array}$

The XOQDOQ program generates four sets of atmospherlc transport factors:

- average annual atmospheric dilution factors which are not corrected for cloud depletion or radloactive decay

- dilution factors which are only corrected for decay assuming a 2.26day half-life

- dilution factors which are corrected for depletion and for decay assuming an 8-day half-life

- relative deposition per unit area.

These factors were used to estimate the dose from airborne releases using methods similar to the NRC GASPAR program (Eckerman 1976). The transport factors used this year were the same as those used for the prevlous estimates. The assumptions used in the calculation of these transport factors were as follows:

- 50-m source height wth no correction for plume rise or building wake effects

- semi-Infinite cloud model with sector-average, Gaussian-plume dispersion

- no correction for terrain helght variation.

Since information about helght and locations at each site for the releases given in Tichler and Benkovitz, (1981) was unavallable, a single generic helght of $50 \mathrm{~m}$ was used at each slte for the release point. Because the heights and locations of releases are uncertaln, estimates of 
dose to persons llving with in $2 \mathrm{~km}$ of the site could be in serlous error; only persons living between 2 to $80 \mathrm{~km}$ from the site were included in the dose estimates.

\section{POPULATION}

The population distribution with in 2 to $80 \mathrm{~km}$ around each site was determined by using a computer program and data base derived from the 1970 census. The program and data base were developed by the Department of Commerce and subsequently adopted by the Environmental Protection Agency (EPA) for use with population exposure problems (Athey, Tell and Janis 1974, Hill (977).

The population data base used was an edited and compressed version of the 1970 Census Bureau's Master Enumeration District List with Coordinates. it contains housing and population counts for each census enumeration district and the geographic coordinates of the population centrold for each district. Using a modifled version of the EPA program and the data base, the population in each of the 160 segments around each site was estimated from a distance of 2 to $80 \mathrm{~km}$. The populations for 1979 were estlmated using the "net increase" factors by state over the population values for 1970 as given in Statistical Abstract of the United States, 1980 (Table 11).

\section{FOOD PRODUCTION YERSUS FOOD CONSUMPTION}

The total food production for the region within $80 \mathrm{~km}$ around each site was the product of the NRC state-wlde productivity figure for each state and a site productivity factor. At some sites this total production may be more or less than the total consumption; i.e., population times average individual consumption (see Table A-1 for generlc consumption rates). When production was more than consumption for a site, it was assumed that all persons in the 2-to-80-km region ate contaminated food; when production was less than consumption, it was assumed that dilution would occur because uncontaminated food would be shipped into the area from outside. Thus, the calculated doses for a particular tood type were reduced in proportion to the ratio of production $\div$ consumption (production/consumption < 1 ).

The dose to persons outside the $80-\mathrm{km}$ limit from tood shipped out of the reglon, in the case of production being greater than consumption, is not included in this report because it is concerned only with the dose within the $80-\mathrm{km}$ radius. These production/consumption factors are given as footnotes to the tables showing airborne dose commitment in the site summary Section for reference. 
DRINKING WATEB

The population between 2 and $80 \mathrm{~km}$ of each plant slte exposed to drinking water contaminted with released radionuclldes was generally obtained from the environmental statement (ES) for the plant. For all sltes located on salt water, It was assumed that no dose was recelved from drinking water. The consumption rates used are given in Table A-l for drinking water.

The radionucl ide concentration in the drinking water consumed by a population downstream from a site was usually estimated assuming $100 \%$ mixing of the plant effluent w ith the river. For lakes, an overall dilution factor was estlmated from dilution factors given in the ES for each population center along the shore (within $80 \mathrm{~km}$ ) that consume the contaminated lake water. These individual factors were welghted by population and averaged to obtain an effective dilution factor for the total population exposed to contaminated drinking water.

\section{AOUATIC FOOD}

Wherever possible, the fish-catch data from the plant ES were used to estimate aquatic food consumption rates for the population living within the region. When these data were not found in the ES or were considered unrealistic, the generic values of Table A-1 were used.

The average radionucl ide concentration of the waters in which this food was harvested was estlmated assuming an additional dilution over the effluent flow from the reactor. For rivers, it was assumed that the fish were caught in waters in which the plant effluent was completely diluted. For lakes, an additional factor as given In the ES was used; when none was given in the ES, a generlc value of 0.01 was used. For ocean and bay sltes, a generic value of 0.001 and 0.002 was used for $f$ Ish and invertebrates, respectively, if the ES ylelded no values for these parameters. Invertebrates were not assumed to be caught in sufficlent quantity at freshwater sites ( $r$ iver and lake) to affect the population dose and therefore were not included in the dose calculation. Any exceptions to these general guldelines are explained in the footnotes to the individual site summaries.

\section{TECHNJCAL_NOTES}

The calculations leading to the dose estlmates contained in this report were generated from recent versions of computer programs originally documented in Baker, et. al (1977). The revised programs were written in BASIC-80 and run on an ALTOS ACS8000 microcomputer operating under the MP/M operating system. The text and tables of the report were typed on an NEC 7710 printer in Dual Gothic Legal 12B font. 


\section{APPENDIX REFERENCES}

Athey, T. W., R. A. Tell and D. E. Janis. 1974. The Use of an Automated Population Data Base in Population Exposure Calculation, CONF 741018. Proceedings of Symposium on Population Exposures. NTIS, Springfleld, VA.

Baker, D. A., J. K. Soldat and E. C. Watson. 1977. Repulation= Dose Commitments Due to Radioact lve Releases from Nuclear Power Plant Sites in 1975, PNL-2439. Pacific Northwest Laboratory, Richland, WA.*

Eckerman, K. 1976. GASPAR DOSE CODE (Computer Listing). U.S. Nuclear Regulatory Commission, Washington, D.C.

HIII, E. R. 1977. Calculation of Population Distribution Using a Computer Data Base, BNWL-2395. Pacific Northwest Laboratory, Richland, WA.

Hoenes, G. R., and J. K. Soldat. 1977. Age-Speclfic Radiation Dose comm tment Factors for a One-Year Chronle lntake, NUREG-0172. U.S. Nuclear Regulatory Commission, Washington, D.C.*

Bopulation Estimates and Projections, Current Population Reports. 1975. Series P-25, No. 541. U.S. Department of Commerce, Soclal and Economic Statistics Administration, Bureau of the Census.

Regulatory Guide 1.109. 1977. Calculation of Annual Deses to Man from Rout ine Releases of Reactor Effluents for the Purpose of Evaluating Compliance with 10 CFR Part 50, Appendix 1. Revision 1. U.S. Nuclear Regulatory Commission, Wash Ington, D.C.

Sagendort, J. F., and J. T. Goll. 1977. X00D00--Program for the Meteorologlcal Evaluation of Reut ine Effluent Releases at Nulcear Power Stations, NUREG-0324. U.S. Nuclear Regulatory Comm Ission, Washington, D.C. **

Statistical Abstract of the United States: 1980. 101 st edition. U.S. Department of Commerce, Bureau of the Census, Washington, D.C.

Tichler, J. and C. Benkovitz. 1981. Radloactive Materials Released from Nuclear Power Plants, Annual_Report 1979, NUREG-CR-2227, BNL-NUREG51416. U.S. Nuclear Regulatory Commission, Washington, D.C.*

* Avallable for purchase from the National Technical Information Service, Springfield, VA 22161.

* Avallable free upon written request to the Division of Technical Informa tion and Document Control, U.S. Nuclear Regulatory Commlssion, Washington, D.C. 20555. 

NUREG/CR-2850 Vol. I

PNL-4421

\title{
DISTRIBUTION
}

No. of

Copies

\author{
DOE Technical Information Center \\ Oak Ridge, TN 37830 \\ MR Beebe \\ Offlce of Resource Management \\ Nuclear Regulatory Commission \\ Washington, DC 20555 \\ 50 Pacific Nor thwest Laboratory \\ P.0. Box 999 \\ Richland, WA 99352 \\ DA Baker (40) \\ RA Peloquin \\ WA Glass \\ EC Watson/JB Brown \\ Publishing Coordinator (2) \\ Technical Information (5)
}





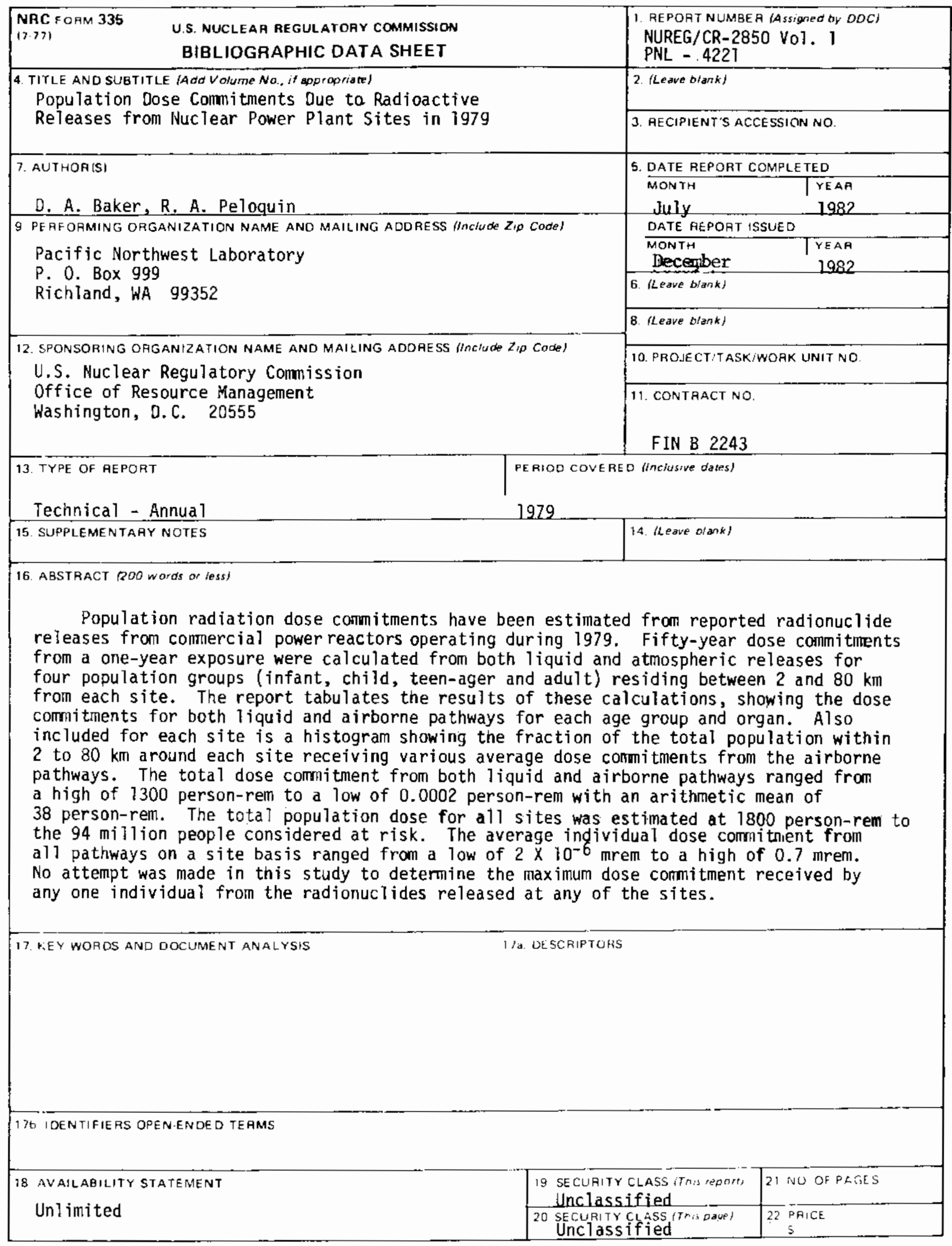


